\title{
STATISTICALLY STEADY MEASUREMENTS OF RAYLEIGH-TAYLOR MIXING IN A GAS CHANNEL
}

\author{
A Dissertation \\ by \\ ARINDAM BANERJEE \\ Submitted to the Office of Graduate Studies of \\ Texas A\&M University \\ in partial fulfillment of the requirements for the degree of \\ DOCTOR OF PHILOSOPHY
}

August 2006

Major Subject: Mechanical Engineering 


\title{
STATISTICALLY STEADY MEASUREMENTS OF RAYLEIGH-TAYLOR MIXING IN A GAS CHANNEL
}

\author{
A Dissertation \\ by \\ ARINDAM BANERJEE
}

Submitted to the Office of Graduate Studies of Texas A\&M University

in partial fulfillment of the requirements for the degree of

DOCTOR OF PHILOSOPHY

Approved by:

Chair of Committee, Malcolm J. Andrews

Committee Members, Ali Beskok

Gerald Morrison

Othon Rediniotis

Head of Department, Dennis O'Neal

August 2006

Major Subject: Mechanical Engineering 


\begin{abstract}
Statistically Steady Measurements of Rayleigh-Taylor Mixing in a Gas Channel.

(August 2006)

Arindam Banerjee, B.E., Jadavpur University;

M.S., Florida Institute of Technology

Chair of Advisory Committee: Dr. Malcolm J. Andrews
\end{abstract}

A novel gas channel experiment was constructed to study the development of high Atwood number Rayleigh-Taylor mixing. Two gas streams, one containing air and the other containing helium-air mixture, flow parallel to each other separated by a thin splitter plate. The streams meet at the end of a splitter plate leading to the formation of an unstable interface and of buoyancy driven mixing. This buoyancy driven mixing experiment allows for long data collection times, short transients and was statistically steady. The facility was designed to be capable of large Atwood number studies of $A_{\mathrm{t}} \sim 0.75$. We describe work to measure the self similar evolution of mixing at density differences corresponding to $0.035<A_{\mathrm{t}}<0.25$. Diagnostics include a constant temperature hot-wire anemometer, and high resolution digital image analysis. The hot-wire probe gives velocity, density and velocity-density statistics of the mixing layer. Two different multi-position single-wire techniques were used to measure the velocity fluctuations in three mutually perpendicular directions. Analysis of the measured data was used to explain the mixing as it develops to a self-similar regime in this flow. These measurements are to our 
knowledge, the first use of hot-wire anemometry in the Rayleigh-Taylor community. Since the measurement involved extensive calibration of the probes in a binary gas mixture of air and helium, a new convective heat transfer correlation was formulated to account for variable-density low Reynolds number flows past a heated cylinder. In addition to the hot-wire measurements, a digital image analysis procedure was used to characterize various properties of the flow and also to validate the hot-wire measurements. A test of statistical convergence was performed and the study revealed that the statistical convergence was a direct consequence of the number of different large three-dimensional structures that were averaged over the duration of the run. 


\section{ACKNOWLEDGEMENTS}

I would like to thank my research advisor, Prof. Malcolm J. Andrews, for his guidance, support and advice during the course of this work. This work has been funded by the US Department of Energy under contract number DE-FG03-02NA00060. I also wish to thank Wayne Kraft for his help in running the experiments and our numerous discussions about the various diagnostics used in this work. Special thanks are also due to Nicholas Mueschke, Michael Peart and Gopinath Subramanian for their help in construction of the facility.

I would also like to thank my parents for giving me the love, values and virtues in life. Their encouragement over the years has been the motivation in getting a doctoral degree. Special thanks, love and appreciation are also due to my wife, Atrayee, for her love, encouragement, support and being a part of this roller coaster life of a graduate student. I wish her all the best for her dissertation work. I am also thankful to my parents-in-law for being supportive over the years. Lastly and most importantly, I would like to thank Dr. Kunal Mitra at Florida Tech for giving me the opportunity to pursue higher studies in the United States and motivating me to pursue a career in research and teaching. 


\section{TABLE OF CONTENTS}

Page

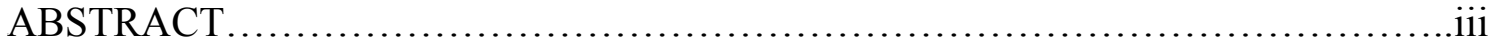

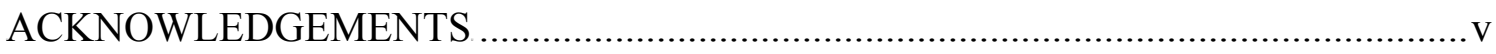

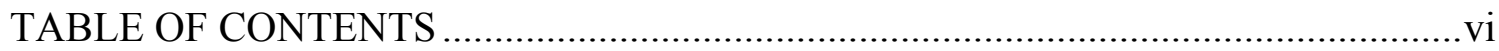

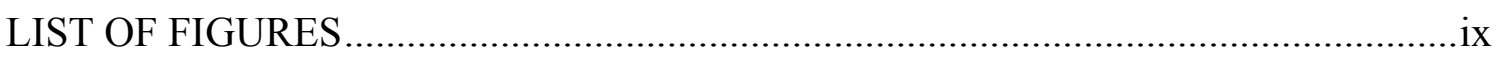

LIST OF TABLES …....................................................................................... xiii

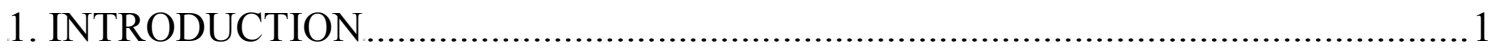

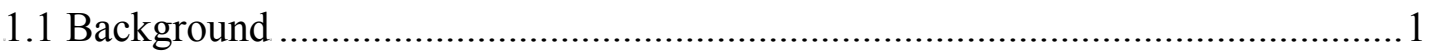

1.2 Previous Rayleigh-Taylor Experiments ........................................................5

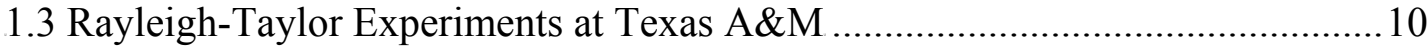

1.4 Hot-Wire Anemometry - Advantages and Limitations ....................................... 17

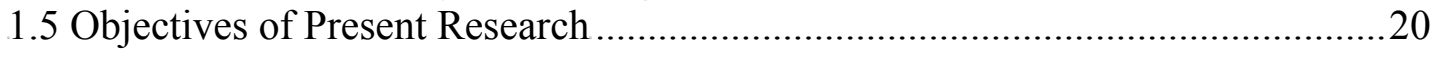

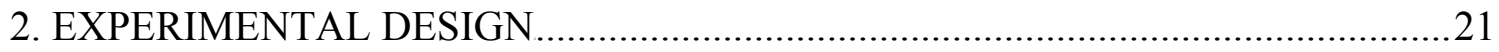

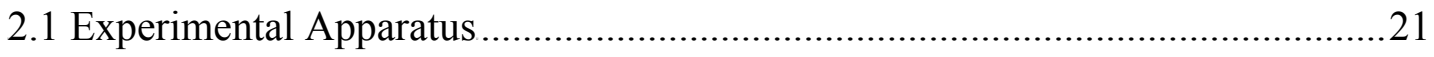

2.2 Mass Flow Rate Calibration....................................................................26

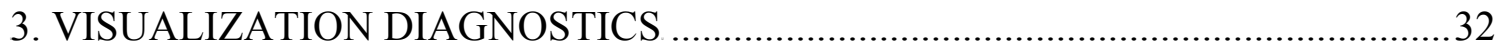

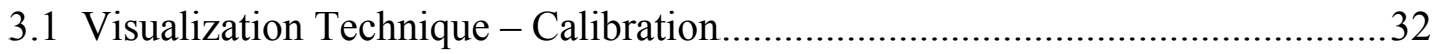

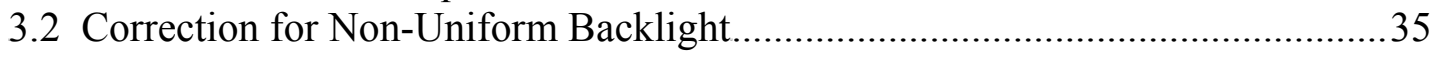

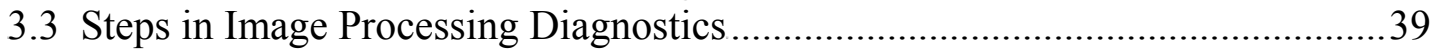

3.4 Qualitative Measurements....................................................................... 41

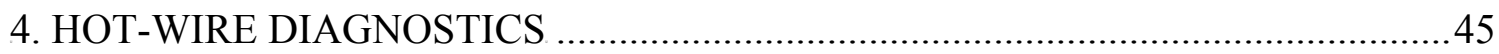

4.1 Constant Temperature Anemometry (CTA) for Measuring Velocity

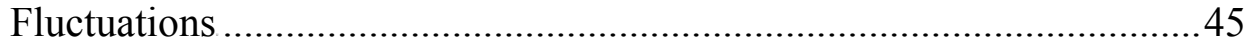

4.1.1 Calibration of a Single-Normal Hot-Wire Probe ..................................48

4.1.2 Hot-Wire Calibration Equations........................................................51

4.2 Constant Current Anemometry (CCA) for Measuring Temperature Fluctuations 
4.2.1 The Need for a CCA Unit .................................................................5

4.2.2 The Cold (Resistance) Wire Probe .........................................................5 59

4.3 Single Wire Measurements ............................................................................6 61

4.3.1 Multi-Position Single Wire Technique .............................................61

4.3.2 Multi-Position Multi-Overheat Single Wire Technique.........................66

5. CONVECTION CORRELATIONS FOR A HEATED WIRE .................................. 84

5.1 Heat Transfer Correlations from Heated Wires at Low Reynolds Number.........84

5.2 Behavior of Hot-Wire/Film in Gas Mixtures ....................................................... 90

5.3 Effect of Temperature Jump on the Heat Transfer Coefficient...........................91

5.4 Convective Correlations for Binary Air-Helium Mixture.................................. 94

5.4.1 Properties of Gas Mixtures................................................................97

5.4.2 Effect of Binary Air-Helium Mixture ..................................................99

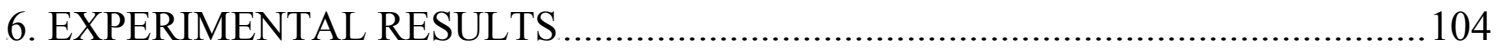

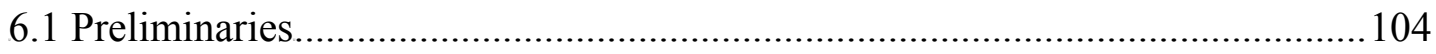

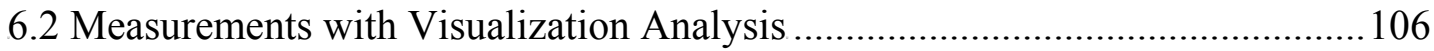

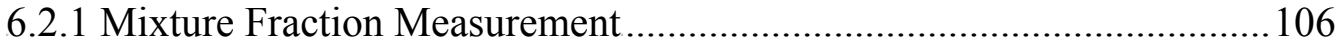

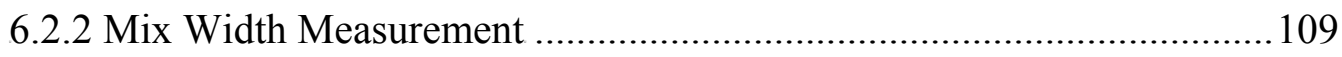

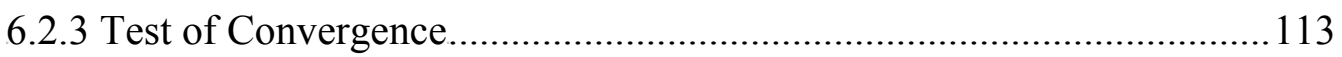

6.2.4 Growth Constant $(\alpha)$ Measurement................................................... 116

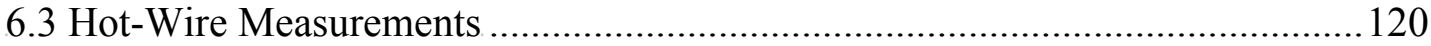

6.3.1 Measurements with Multi-Position SN-Wire Technique....................... 120

6.3.2 Measurements with Multi-Position Multi-Overheat Technique ............. 124

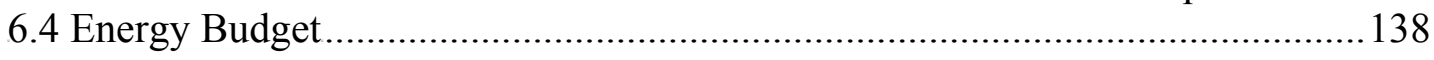

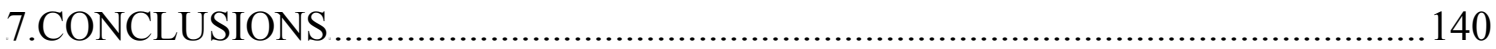

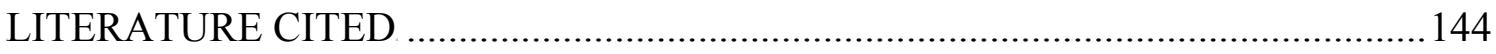

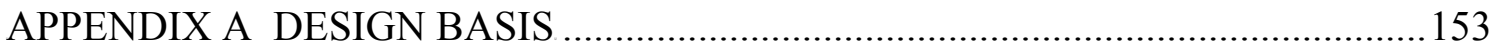

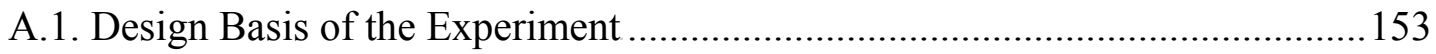

A.2 Gravity Current Effects in the Gas Channel............................................... 156

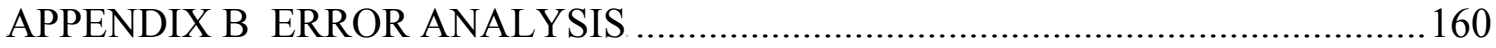

B.1. Error in Mass Flow Rate Measurement........................................................ 160

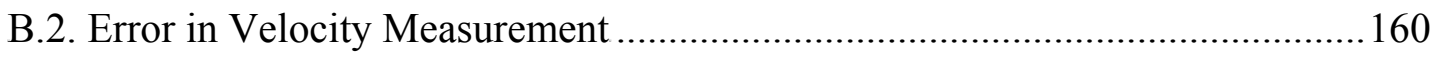

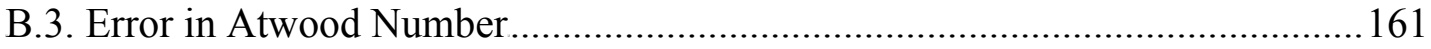




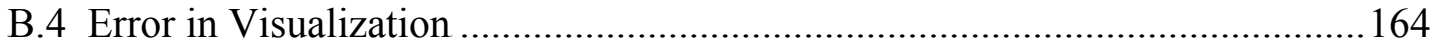

B.5 Error in Hot-Wire Measurement.................................................................... 165

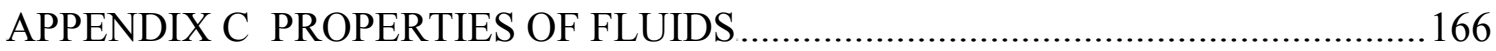

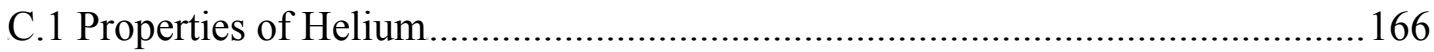

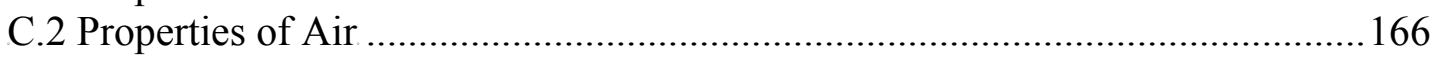

APPENDIX D CALIBRATION CURVE FITS \& ANALYSIS SOFTWARE.............167

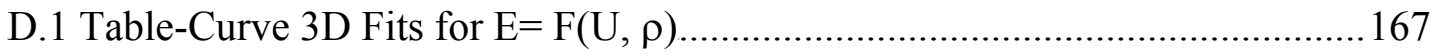

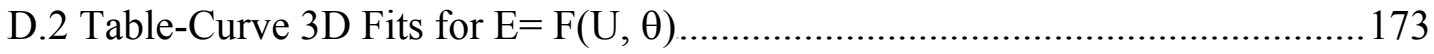

D.3 Matlab routine to solve for density-velocity correlations .............................179

APPENDIX E. TWO FLUID INTERFACE IN ABSENCE OF SHEAR \& BUOYANCY …..................................................................... 181

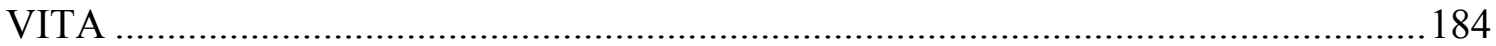




\section{LIST OF FIGURES}

FIGURE

Page

1.1 Various stages of evolution of Rayleigh-Taylor instability ............................... 2

1.2 Schematic of water channel facility at Texas A\&M University .........................11

1.3 Photograph of the water channel experiment,with nigrosene dye added to

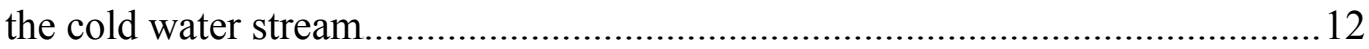

1.4 Photograph of gas channel facility at Texas A\&M University ...........................15

1.5 Gas channel images of evolution of the R-T instability for $A_{\mathrm{t}} \# 0.04 \ldots \ldots \ldots \ldots \ldots \ldots . . . . .16$

2.1 Schematic of high Atwood number helium-air gas channel facility used for the experiments

2.2 Schematic of flow metering unit for a constant mass flow rate of helium. 27

2.3 Calibration of mass flow rate of helium for different Atwood numbers 28

3.1 Intensity as a function of height for calibration wedge 34

3.2 Histogram for raw image

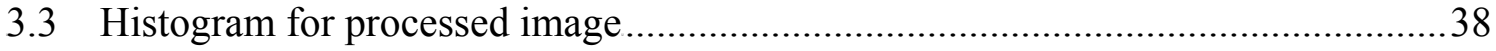

3.4 View of the mixing process in the channel at (a) $A_{\mathrm{t}} \# 0.04\left(U_{\mathrm{m}}=50 \mathrm{~cm} / \mathrm{s}\right)$ and (b) $A_{\mathrm{t}} \# 0.097\left(U_{\mathrm{m}}=85 \mathrm{~cm} / \mathrm{s}\right)$.

3.5 Close-up view of the three dimensional plumes across the channel for

(a) $A_{\mathrm{t}} \# 0.04\left(U_{\mathrm{m}}=50 \mathrm{~cm} / \mathrm{s}\right)$ and (b) $A_{\mathrm{t}} \# 0.097\left(U_{\mathrm{m}}=85 \mathrm{~cm} / \mathrm{s}\right)$

3.6 Movie of the mixing process for $A_{\mathrm{t}} \# 0.259\left(U_{\mathrm{m}}=1.2 \mathrm{~m} / \mathrm{s}\right) \ldots \ldots \ldots \ldots \ldots \ldots \ldots \ldots . .44$

4.1 The constant temperature anemometer (CTA) circuit.......................................46

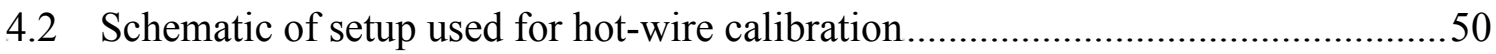


FIGURE

4.3 Variation in $\varepsilon_{\mathrm{u}}$ (goodness of fit) with exponent in power law relationship from $\mathrm{SN}$-wire calibration data in air at overheat ratio 0.6 over a velocity range of $0.2-3 \mathrm{~m} / \mathrm{s}$

4.4 Hot-wire calibration data (King's law fit) in air at overheat ratio 1.9

$\left(\Delta T=257.14^{\circ} \mathrm{C}\right)$ and $1.6\left(\Delta T=171.43^{\circ} \mathrm{C}\right)$.

4.5 Variation in King's law constants for different volume fractions of helium in a binary air-helium mixture. 56

4.6 Calibration curve for cold wire (resistance wire) probe. 60

4.7 Co-ordinate system for measurements and various orientations of hot-wire used for measurements

4.8 Wire orientations for multi-overheat multi-position technique 71

4.9 Velocity- density calibration for wire 1 at overheat ratio 1.9 74

4.10 $d E / d U$ for wire 1 at overheat ratio 1.9. 75

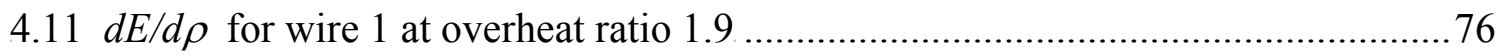

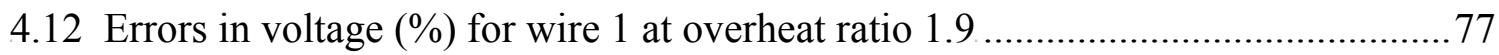

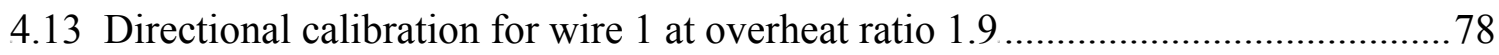

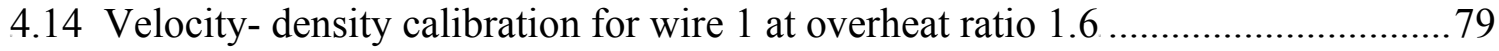

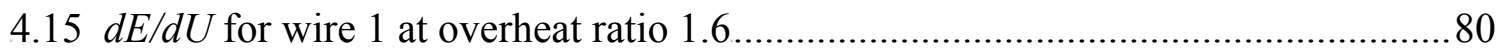

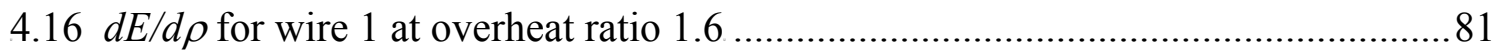

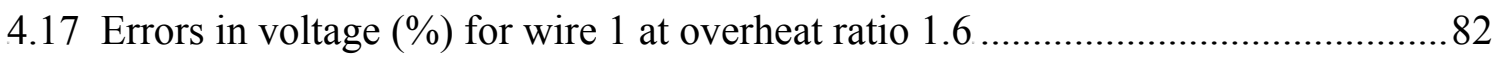

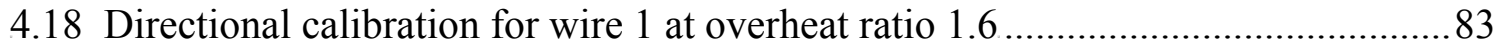

5.1 Hot-wire calibrations in different volume fractions of helium in a binary air-helium mixture. .96

5.2 Absolute viscosity and thermal conductivity of air-helium mixture .98 
FIGURE Page

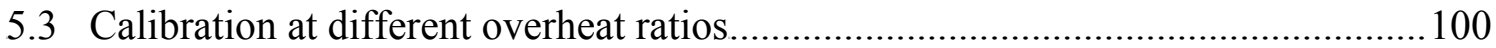

5.4 Heat transfer correlation in a binary gas mixture of air and helium..................... 103

6.1 Close-up view of the three dimensional plumes across the channel for

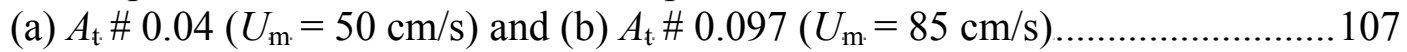

6.2 Contour levels $(5 \%, 20 \%, 50 \%, 80 \%$ and $95 \%)$ plotted on average image

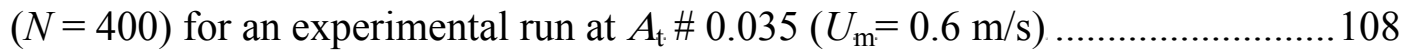

6.3 Mixture fraction distributions across the mixing layer for $A_{\mathrm{t}} \# 0.035 \ldots \ldots \ldots \ldots \ldots \ldots \ldots . . .110$

6.4 Mixture fraction distributions across the mixing layer for $A_{\mathrm{t}} \# 0.259 \ldots \ldots \ldots \ldots \ldots \ldots \ldots . . . . .111$

6.5 Effect of number of images in average on the mixing width ............................. 112

6.6 Percentage $(\%)$ root mean square error of density as a function of number of images $(16 \times 16$ interrogation window $)$

6.7 Comparison of growth constant $\left(\alpha_{b}\right.$ ) measured with image diagnostics (gas channel) and PIV (water channel)

6.8 Comparison of growth constant $\left(\alpha_{b}\right.$ ) obtained by image analysis (at gas channel) with LEM (Linear Electric Motor) experimental measurement of Dimonte \& Schneider (1996)

$6.9 \alpha$ at the centerline plotted as a function of $\tau$.

6.10 Density profiles measured with the multi-position multi-overheat hot-wire technique.

6.11 Molecular mix parameters across the mix at $\tau=1.986\left(A_{\mathrm{t}} \# 0.04\right.$, $x=1.75 \mathrm{~m}$ from the splitter plate).

6.12 Time evolution of scalar turbulence intensity and mix parameters measured at the centerline $\left(A_{\mathrm{t}} \# 0.04\right)$.

6.13 Comparison of measured $\alpha$ by different techniques (Hot-wire: MPMO: Multi-Position Multi-Overheat Method; MP: Multi-Position Method) at $A_{\mathrm{t}} \# 0.04$. Water channel measurement was at $A_{\mathrm{t}} \# 7.5 \times 10^{-4}$ 
FIGURE

6.14 Velocity correlations across the mix at $x=1.75 \mathrm{~m}(\tau=1.986)$ for an experimental run at $A_{\mathrm{t}} \# 0.04$

6.15 Ratio of $v^{\prime} / u^{\prime}$ across the mix at $x=1.75 \mathrm{~m}(\tau=1.986)$ for an experimental run at $A_{\mathrm{t}} \# 0.04$

6.16 Evolution of primary transport term for $A_{\mathrm{t}} \# 0.04$

6.17 Profile of $\left\langle\rho^{\prime} v^{\prime}\right\rangle$ and $\left\langle\rho^{\prime} u^{\prime}>\right.$ across the mix at $\tau=1.986$ for $A_{\mathrm{t}} \# 0.04$

A.1 Stable configurations $\left(\rho_{1 .}>\rho_{2}\right)$ in a square box $(1 \mathrm{~m} \times 1 \mathrm{~m})$ used to calculate conversion from potential energy to kinetic energy. 158

E.1 Two fluid interface in absence of shear and density gradient $\left(U_{\mathrm{m}}=0.6 \mathrm{~m} / \mathrm{s}\right) \ldots \ldots . .182$

E.2 Two fluid interface in absence of shear and density gradient $\left(U_{\mathrm{m}}=0.84 \mathrm{~m} / \mathrm{s}\right) \ldots . .182$

E.3 Two fluid interface in absence of shear and density gradient $\left(U_{\mathrm{m}}=1.2 \mathrm{~m} / \mathrm{s}\right) \ldots \ldots .183$

E.4 Two fluid interface in absence of shear and density gradient $\left(U_{\mathrm{m}}=1.65 \mathrm{~m} / \mathrm{s}\right) \ldots . .183$ 


\section{LIST OF TABLES}

TABLE

Page

1.1 List of R-T experiments since 1950 and their respective authors, fluids used, Atwood number range obtained, mode of initial perturbation, diagnostics and experimental run time.

1.2 Comparison of design parameters between gas channel and water channel.

1.3 Comparisons between Hot-Wire Anemometry (HWA), Particle Image Velocimetry (PIV) and Laser Doppler Velocimetry (LDV) on the basis of the requirements in the gas channel facility.....

2.1 List of flow-straighteners and meshes in the inlet section of the facility. 25

2.2 Calibrated mass flow rates for different orifice 29

3.1 Camera (Canon Powershot A80) settings at different Atwood numbers

4.1 Sample operating parameters for $\mathrm{SN}$ wire coupled to a mini-CTA unit (overheat ratio $=1.6$ ).

4.2 Curve fits in the velocity range $0-3 \mathrm{~m} / \mathrm{s}$ (calculated at overheat ratio 1.6 )

4.3 Various measurement orientations for multi-position measurement technique .65

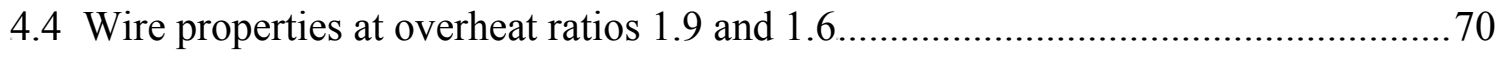

4.5 Various measurement orientations for multi-position measurement technique.........72

5.1 Convection heat transfer correlations for flow over a heated cylinder. .88

5.2 List of convection heat transfer correlations involving hot-wire anemometry (small cylinders)

5.3 Sample calculations for various correlation parameters for air. 102

6.1 List of imaging and hot-wire experiments 105 
6.2 Velocity fluctuations $(\mathrm{m} / \mathrm{s})$ measured at $A_{\mathrm{t}} \# 0.035\left(U_{\mathrm{m}}=0.6 \mathrm{~m} / \mathrm{s}\right) \ldots \ldots \ldots \ldots \ldots \ldots \ldots \ldots \ldots \ldots \ldots \ldots \ldots \ldots . . .122$

A.1 Minimum flow velocity to satisfy parabolic approximation in gas channel..........155

A.2 Vertical velocity ( $v$ ) of the gravity wave and mean flow velocity $U_{\mathrm{m}}$. corresponding to different run conditions at a different Atwood numbers $(L=0.6 \mathrm{~m})$...

B.1 Uncertainty in Atwood Number for various experimental run conditions. 163 


\section{INTRODUCTION}

\subsection{Background}

Rayleigh-Taylor (R-T) instability may be induced when a heavy fluid is placed over a light fluid in a gravitational field. If the planar surface between the two fluids is disturbed with a perturbation of finite amplitude, the disturbances are driven by buoyancy and develop as R-T instability (Rayleigh 1884; Taylor 1950). The interface becomes distorted with time and the wavelengths associated with the initial disturbance interact between themselves causing a mingling process to degenerate into a turbulent mix. Development of the mix was divided by Youngs (1984) into three successive regimes. The mix starts with an initial exponential growth of infinitesimal perturbations that correspond with linear stability analysis. At amplitude about one-half of the wavelength, the linear growth regime of the instability saturates and the perturbation speed settles to at a constant rate. Thereafter, longer wavelengths overtake due to their continuing exponential growth, a phenomenon referred to as "bubble competition" (Emmons et al. 1960). Eventually, a self-similar R-T mix layer is formed through mode interaction and successive wavelength saturation. The three stages have been illustrated in Figure 1.1.

Once at self similarity, and with loss of memory of the initial conditions, dimensional analysis suggests that the mixing half-width grows quadratically with time according to the relation, $h \propto g t^{2}$, where, $t$, is the time and $g$, the acceleration due to gravity. 

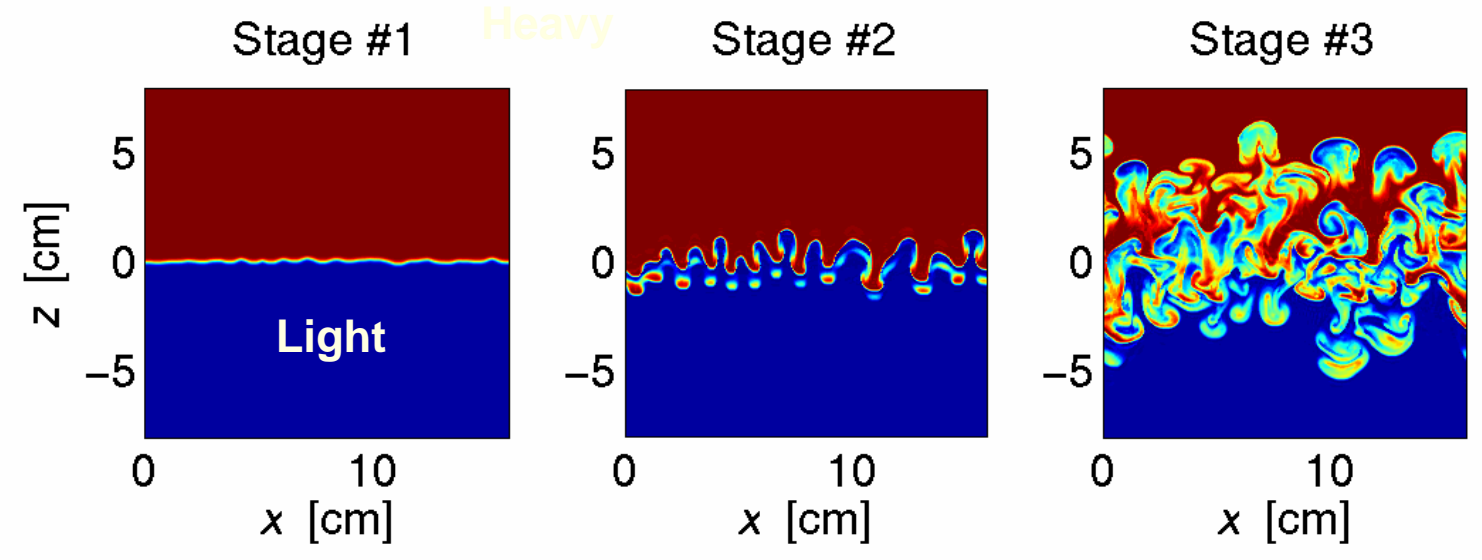

Figure 1.1 Various stages of evolution of Rayleigh-Taylor instability.

Stage 1 - Exponential growth of infinitesimal perturbations; Stage 2 - Saturation of the initial perturbations; and Stage 3 - Bubble competition. Images are taken from 3DDNS with a resolution of $256 \times 128 \times 256$ by Mueschke $(2004)$. 
However, experiments and computations (Anuchina et al. 1978 ; Youngs 1984) suggest that a more complete description was given by:

$$
h_{b, s}=\alpha_{b, s} A_{t} g t^{2} \quad(b: \text { bubble; } s: \text { spike })
$$

where the Atwood number, $A_{\mathrm{t}}$, denotes the governing parameter of the flow defined by $A_{t} \equiv\left(\rho_{1}-\rho_{2}\right) /\left(\rho_{1}+\rho_{2}\right) ; \rho_{1}$ and $\rho_{2}$ are the densities of air (heavy fluid) and airhelium mixture (light fluid) employed in the present work; $h_{b}$ and $h_{\mathrm{s}}$ were the heights (above/below the density interface) of the "rising" bubbles and the "falling" spikes respectively; $\alpha_{b}$ and $\alpha_{s}$ denotes the growth rate constants (for the bubbles and spikes) which was to be determined. For low Atwood numbers $(<0.1)$, the mix is symmetric $\left(h_{\mathrm{b}}\right.$ $=h_{\mathrm{s}}$ ) and $\alpha$ was usually taken as a constant, i.e. $\alpha_{\mathrm{b}}=\alpha_{\mathrm{s}}=\alpha$ (Dimonte 1999; Ramaprabhu \& Andrews 2004). However, for high Atwood numbers $(\geq 0.1)$, the mix is no longer symmetric about the density interface $\left(h_{\mathrm{s}}>h_{\mathrm{b}}\right)$. The values of $\alpha$ is found to be different $\left(\alpha_{\mathrm{b}}<\alpha_{\mathrm{s}}\right)$ with $\alpha_{\mathrm{s}}$ being a function of the Atwood number and $\alpha_{\mathrm{b}}$. was approximately constant (Dimonte 1999; Dimonte \& Schneider 1996). Equation (1.1) for $h$ was obtained by Youngs (1984) by using a nonlinear extension of the linear stability theory (Chandrasekhar 1961).

R-T flows represent a canonical fluid flow that encompasses the laminar, transition and turbulent flow regimes. A complete understanding of R-T flows is desired because of the broad impact such flows have in nature and a variety of applications, in particular, in buoyancy and shock driven instabilities which occur during the implosion phase of Inertial Confinement Fusion (ICF) process (Lindl 1998; Roberts et al. 1980). An ICF process involves high power laser or x-ray bombardment of target fuel capsules 
(deuterium-tritium pellets). Implosion of the pellets to a super-dense state necessary for thermonuclear burn requires a spherical symmetry (Clarke et al. 1973). Surface imperfections in the pellets and drive asymmetries lead to unavoidable departures from this spherical symmetry which gives rise to the hydrodynamic instabilities. The acceleration phase of an ICF capsule is Richtmeyer-Meshkov (R-M) unstable, while the late-time deceleration phase is Rayleigh-Taylor unstable (Lindl 1998; Roberts et al. 1980). The R-M Instability is an impulsively driven variant of the R-T Instability (Brouillette 2002; Meshkov 1969; Richtmyer 1960). The growth of the R-T driven mixing layer has been shown to be the limiting factor for the yield of the ICF process (Atzeni \& Meyer-ter-Vehn 2004; Betti et al. 2001; Lindl 1998).

In astrophysics, the formation of fast optical filaments in a young supernova has been attributed to Rayleigh-Taylor instability as the expanding sphere sweeps up interstellar material (Gull 1975). It was assumed that the limiting factor in the creation of the heavy interstellar elements in collapsing stars was the growth of the mixing layer formed by adverse density stratification in its gravitational field (Smarr et al. 1981). R-T generated turbulence also occurs in geophysical formations like salt domes and volcanic islands (DiPrima \& Swinney 1981); in deep-sea ocean currents and in rivers and estuaries (Cui \& Street 2004; Molchanov 2003). The breakup of fuel droplets in high speed flows have also been found to be R-T unstable (Marmottant \& Villermaux 2004; Thomas 2003). Experiments performed to study atomization of a liquid jet when a fast gas stream blows parallel to its surface show that the liquid destabilization proceeds from a two-stage mechanism: a shear instability first forms waves on the liquid. The 
transient acceleration experienced by the liquid suggests that a Rayleigh-Taylor type of instability is triggered at the wave crests, producing liquid ligaments which further stretch in the air stream and break into droplets (Marmottant \& Villermaux 2004). Studying such complex flows, which consist of various ranges of length-scales and timescales, represents a grand challenge to the turbulence community. Thus, this research experimentally studies the growth and turbulence of R-T driven mixing over a wide range of Atwood numbers. A literature review of R-T experiments over the years is provided in Section 1.2 to analyze the scope and limitations of the various R-T experimental facilities available at present (also see Table 1.1).

\subsection{Previous Rayleigh-Taylor Experiments}

The first mention of the instability comes in the early work of Lord Rayleigh (1884). G. I. Taylor (1950) put forth a first order theory to explain the linear (early time) regime of the instability. D. J. Lewis (1950) performed a series of experiments to test the validity of Taylor's proposed theory. The experimental apparatus consisted of a large air reservoir connected to a rectangular tube. A flange holding a thin shellac diaphragm was placed at the center of the tube. The bottom of the tube was fitted with a foil. Supported on the diaphragm was water. Air pressure in the top reservoir and the bottom tube was adjusted separately and isolated from each other. When the foil at the bottom of the tube was ruptured, the unbalanced pressure drove the liquid down the tube and the R-T instability was observed at the interface. The pressure in the reservoir and the tube was unbalanced to obtain a range of accelerations from $3 \mathrm{~g}$ to $140 \mathrm{~g}$. The height of liquid on the diaphragm varied from 0.375 " to 20 ". High speed shadow photography was used as 
diagnostics and the run time obtained was $\sim 10^{-2}$ seconds. However, both Taylor and Lewis did not take into consideration effects of surface tension and viscosity on the rate of growth of the instability and studied the instability on fluid combinations like airbenzene, air-water and air-glycerin. Allred and Blount (1954) included the effects of surface tension and fluid viscosity in their experiments by choosing a combination of water and n-heptane / isoamyl alcohol / n-octyl alcohol as the working fluids. They used an apparatus similar to that of Lewis (1950). Using these fluid combinations lowered the interfacial tension between the two fluids by a factor of 20 as the other fluid properties remained unchanged. The Atwood number range obtained (Allred \& Blount 1954; Lewis 1950) was between 0.1 and 0.99 .

The R-T instability has also been studied by accelerating an initially stable stratified mixture of two fluids by using a variety of mechanisms like rubber cords (Emmons et al. 1960), bungee/elastic cords (Ratafia 1973) and compressed air (Cole \& Tankin 1973). The objective of these experiments was to excite a particular eigenmode of the surface and then to accelerate the stratified mixture when the initial perturbations had a prescribed amplitude and phase. However, majority of the experiments described above (Cole \& Tankin 1973; Emmons et al. 1960; Ratafia 1973) used a vibrating paddle for generating the initial perturbations which resulted in generation of a wide spectrum of modes instead of a single eigenmode (Popil \& Curzon 1979). The phase of such modes and their amplitudes were variable and thus, the experiments were not repeatable. Popil and Curzon (1979) used a electrostatic generator to accurately generate single-mod 
TABLE 1.1 List of R-T experiments since 1950and their respective authors, fluids used, Atwood number range obtained, mode of initial perturbation, diagnostics and experimental run time

\begin{tabular}{|c|c|c|c|c|c|c|c|}
\hline Year & Authors & Fluids & Atwood \# & Mode & 2D/3D & Diagnostics & Run time \\
\hline 1950 & Lewis & $\mathrm{A} / \mathrm{B}, \mathrm{A} / \mathrm{G} \& \mathrm{~A} / \mathrm{W}$ & 0.99 & $\mathrm{~S}$ & $2 \mathrm{D}$ & Imaging & $\sim 10^{-2} \cdot \mathrm{s}$ \\
\hline 1954 & Allred et. al. & $\mathrm{W} / \mathrm{nH}, \mathrm{W} / \mathrm{OA}, \mathrm{W} / \mathrm{I}, \mathrm{nH} / \mathrm{A}$ & $0.188-0.995$ & S & $2 \mathrm{D}$ & Imaging & $\sim 10^{-2} \cdot \mathrm{s}$ \\
\hline 1960 & Emmons et. al. & $\mathrm{CT} / \mathrm{A} \& \mathrm{M} / \mathrm{A}$ & $0.107,0.997$ & $\mathrm{~S}$ & $2 \mathrm{D}$ & Imaging & $<0.1 \mathrm{~s}$ \\
\hline 1973 & Ratafia & "OA/W & 0.095 & $\overline{\mathrm{S}}$ & $2 \mathrm{D}$ & Imaging & 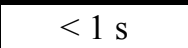 \\
\hline 1973 & Cole \& Tankin & $\mathrm{A} / \mathrm{W}$ & 0.99 & $\mathrm{~S}$ & $2 \mathrm{D}$ & Imaging & $<10^{-2} \cdot \mathrm{s}$ \\
\hline 1979 & Popil \&Curzon & $\mathrm{A} / \mathrm{W}$ & 0.99 & $\mathrm{~S}+\mathrm{M}$ & $2 \mathrm{D}$ & Imaging & $<0.3 \mathrm{~s}$ \\
\hline 1984 & Read & $\mathrm{W} / \mathrm{P}, \mathrm{SI} / \mathrm{P}, \mathrm{EA} / \mathrm{A}$ & $0.231-0.997$ & $\bar{M}$ & $2 \mathrm{D} / 3 \mathrm{D}$ & Imaging & $<10^{-2} \cdot \mathrm{s}$ \\
\hline 1986,1990 & Andrews \& Spalding & $\mathrm{Br} / \mathrm{W}$ & 0.048 & $\mathrm{M}$ & $2 \mathrm{D}$ & Imaging & $\sim 2 \mathrm{~s}$ \\
\hline $1985-2005$ & Jacobs et al. & $\mathrm{A} / \mathrm{W}$ & 0.99 & M & $3 \mathrm{D}$ & Imaging & $<1 \mathrm{~s}$ \\
\hline 1991,1994 & Linden \& Redondo & $\mathrm{Br} / \mathrm{W}$ & $10^{-4}$ to 0.05 & M & $3 \mathrm{D}$ & $\begin{array}{c}\text { Imaging, LIF, } \\
\text { Conductivity } \\
\text { measurements }\end{array}$ & $\sim 3-4 \mathrm{~s}$ \\
\hline 1993, 1999 & Dalziel et al. & $\mathrm{Br} /(\mathrm{W}+\mathrm{P} 2)$ & $2 \times 10^{-3}$ to $7 \times 10^{-4}$ & $\mathrm{M}$ & $3 \mathrm{D}$ & LIF & $\sim 5 \mathrm{~s}$ \\
\hline 1994- 2004 & Andrews et al. & Hot W-Cold W & $10^{-4}$ to $10^{-3}$ & M & $3 \mathrm{D}$ & $\begin{array}{l}\text { Imaging, PIV, } \\
\text { Thermocouples }\end{array}$ & $\sim 600 \mathrm{~s}$ \\
\hline 1996- 2004 & Dimonte \& Schneider & $\mathrm{S} / \mathrm{H}, \mathrm{S} / \mathrm{BT}$, Various & $0.15-0.96$ & $\mathrm{M}$ & $2 \mathrm{D}$ & LIF \& Imaging & $<0.1 \mathrm{~s}$ \\
\hline $1997-2003$ & Kucherenko et al. & $\begin{array}{c}\mathrm{G} / \mathrm{B}, \mathrm{W} / \mathrm{Hg}, \mathrm{W} / \mathrm{Kl}, \\
\mathrm{B} /(\mathrm{W}+\mathrm{G}) / \mathrm{SHS}\end{array}$ & $0.23-0.5$ & M & $3 \mathrm{D}$ & $\begin{array}{l}\text { Pulsed x-ray } \\
\text { photography }\end{array}$ & $<10^{-2} \cdot \mathrm{s}$ \\
\hline $\begin{array}{l}2004- \\
\text { present } \\
\end{array}$ & Andrews and Banerjee & $\mathrm{A} / \mathrm{H}$ & $0.035-0.75$ & M & $3 \mathrm{D}$ & Imaging, Hot-wire & $\sim 300 \mathrm{~s}$ \\
\hline
\end{tabular}

\section{Index for Fluids:}

A: Air, Al: Alcohol, B: Benzene, Br: Brine, BT: Butane, CT: Carbon Tetrachloride, EA: Ethyl Alcohol, G: Glycerin, H: Helium, Hg: Mercury, I: IsoAmyl Alcohol, Kl: Klerichi liquid (Formic-Malonic Acid Talium), M: Methanol, nH: n-Heptane; OA: Octyl Alcohol, P: Pentane, P2: Propan-2-ol, PT: 
standing waves on the water interface. A timing circuit was wired along with the wave generator to control the time of release and subsequent acceleration of the tank. By controlling the number of electrodes in the electrostatic generator, single or multi-mode excitations was induced at the interface and the experiment was thus more repeatable.

A major drawback of the experiments described above was that they were all conducted in narrow long cavities and were two-dimensional. To examine threedimensional turbulent mixing layers, Read (1984) used rockets to accelerate an initially stable stratified mixture downwards. He obtained accelerations of $25 \mathrm{~g}-75 \mathrm{~g}$ by using this method. Jacobs and Caton (1988) accelerated a small volume of water down a vertical tube using compressed air. They used high speed motion picture photography to study 3D Rayleigh-Taylor Instability in a round and square tube with acceleration varying between $5 \mathrm{~g}$ to $10 \mathrm{~g}$. Andrews and Spalding (1990) created an unstable buoyancy gradient by quickly inverting a stable stratified mixture. Linden et al. (1992) and Dalziel et al. (1999) placed a heavy fluid over a light fluid, separated by a thin plate. The plate was withdrawn and buoyancy driven mixing ensued between the two fluids. Recently, Jacobs and Dalziel (2005) studied R-T instability in a system of three fluids using the same technique. The stratification consists of one stable and one unstable interface and was formed by using different salt solutions and fresh water. Kucherenko $(2003 ; 1997)$ used a drop tank technique that was accelerated using a gas gun to achieve accelerations between $100 \mathrm{~g}$ to $350 \mathrm{~g}$. He used an aqueous solution of glycerin and benzene to give Atwood numbers ranging from 0.23 to 0.5 . Diagnostics used included pulsed $\mathrm{x}$-ray photography. Dimonte et al. (1999; 1996) studied turbulent RT growth rates over a 
comprehensive range of Atwood numbers $(0.1304-0.961)$ with constant acceleration using the Linear Electric Motor. Diagnostics involved bi-level LIF (Laser Induced Fluorescence) measurements and backlight photography. However, a major drawback of these experiments was that they all used complicated mechanisms like rockets, fastsliding plates and linear electric motors to generate the R-T instability. In addition, all these experiments were transient studies with short data capture times $(\sim 0.001-5$ second). Thus, a large number of repeated experiments were needed to collect statistical data sets.

Over the past two decades, advances in modeling of variable density turbulence has led to introduction of various turbulence models which includes: spectral transport models (Besnard et al. 1990; 1992; Steinkamp et al. 1995), two-fluid models (Andrews 1986; Youngs 1989) and Reynolds Stress/Bousinesq models (Snider \& Andrews 1996). Validation of predictive turbulent transport models consisting of inhomogeneous, anisotropic and variable-density flows require a priori knowledge of various velocity, density and velocity-density correlations like $\overline{\rho^{\prime 2}}, \overline{\rho^{\prime} u^{\prime}}, \overline{\rho^{\prime} v^{\prime}}, \overline{u^{\prime 2}}, \overline{v^{\prime 2}}$ and $\overline{u^{\prime} v^{\prime}}$. These quantities may be computed from direct numerical simulation (DNS). Cook and Dimotakis (2001) performed DNS of $256^{2} \times 1024$ reaching a Taylor Reynolds number of 100; which was the proposed threshold for mixing transition (Dimotakis 2000). Cook et al. (2004) used a very high resolution large-eddy-simulation $\left(1152^{3}\right)$ to further investigate the asympototic growth of the mixing layer. Such a simulation constitutes one realization of the mixing layer and was a typical state-of-the-art DNS of R-T mixing. 
However, such calculations were limited to low-Reynolds numbers. Thus, there is a need to experimentally determine these quantities.

\subsection{Rayleigh-Taylor Experiments at Texas A\&M}

A water-channel facility, built at Texas A\&M University in the early 1990's addressed these deficiencies (Snider \& Andrews 1994). The facility uses a novel experimental setup which eliminates complex mechanisms like rockets, fast sliding plates, elastic cords, linear electric motors or compressed air cannons. Two streams of fluid, cold water (heavy) on top and hot water (light) at the bottom (see Figure 1.2) flow parallel to each other separated by a thin splitter plate. The streams meet at the end of a splitter plate creating an unstable interface which leads to buoyancy mixing, albeit at small $A_{t} \sim 10^{-4} \cdot-10^{-3}$ (Ramaprabhu \& Andrews 2004; Snider 1994; Snider \& Andrews 1994; 1995; Wilson 2002; Wilson et al. 1999; Wilson \& Andrews 2002). The water channel setup is similar to a shear flow experiment. A combined buoyancy and shear mixing layer can also be obtained if the velocities of the top and bottom streams are different. If the velocities are identical, a buoyancy mixing layer is obtained. The facility allows long run times of $\sim 600$ seconds, thus allowing measurement of higher order statistics.

The basis of the water channel experiment is a Galilean transformation from a moving frame of reference at the mean convective velocity to a transient frame of reference (Snider 1994). For this, the experimental flow should be parabolic, i.e. a oneway characteristic was essential where downstream conditions do not affect the upstream behavior. It has been well established from boundary layer-type assumptions that shear 


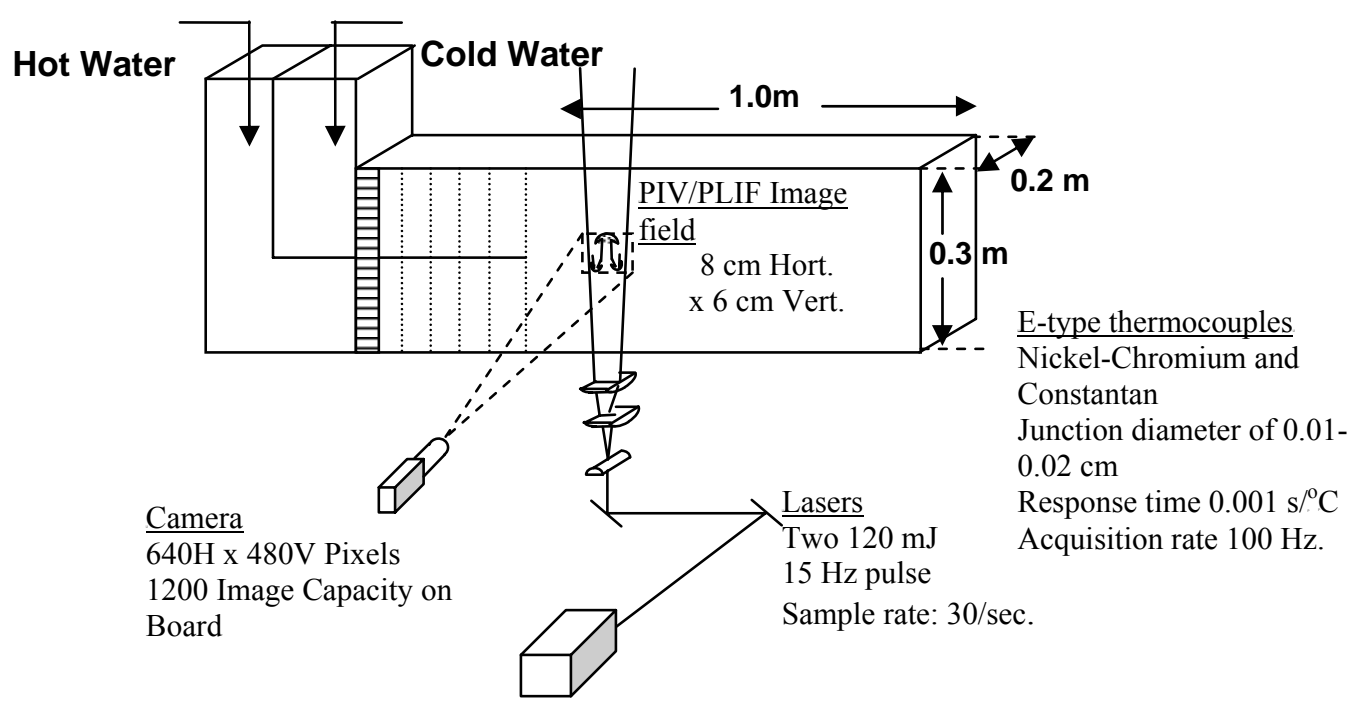

Figure 1.2 Schematic of water channel facility at Texas A\&M University (Ramaprabhu \& Andrews 2004). 


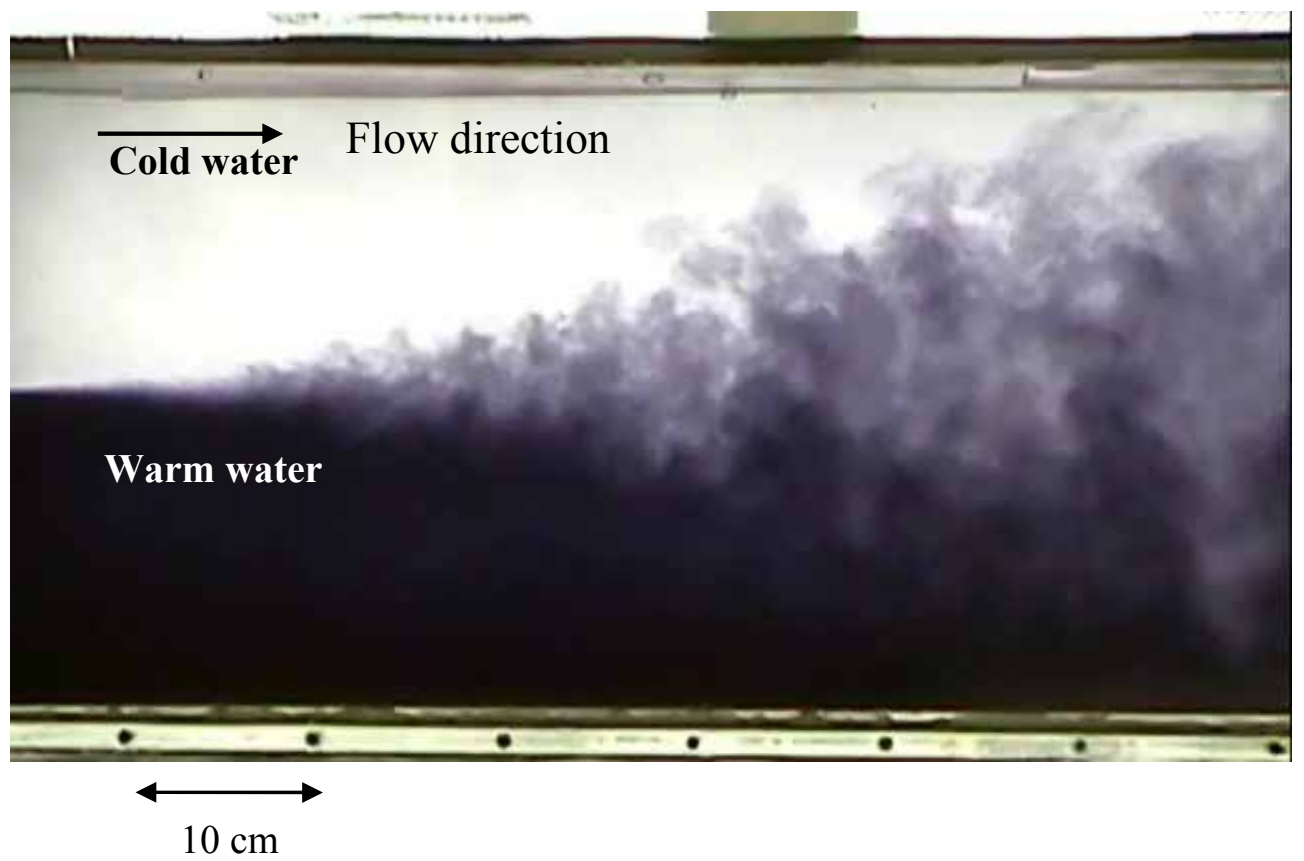

Figure 1.3 Photograph of the water channel experiment, with nigrosene dye added to the cold water stream. The evolution of the mix was quadratic in $x$ (downstream coordinate), with the mix width depending on the Atwood number $\left(A_{t}\right)$, and $g$, the acceleration due to gravity. In this experiment, the distance downstream can be related to time through the Taylor's hypothesis. 
TABLE 1.2 Comparison of design parameters between gas channel and water channel

\begin{tabular}{|c|c|c|}
\hline Parameter & Water Channel & Gas Channel \\
\hline Medium & Hot/Cold Water & Air-Helium \\
\hline Atwood number & 0.001 & 0.75 \\
\hline Dimensions $(L \times B \times H)$ & $1.0 \mathrm{~m} \times 0.3 \mathrm{~m} \times 0.2 \mathrm{~m}$ & $2.0 \mathrm{~m} \times 0.6 \mathrm{~m} \times 0.4 \mathrm{~m}$ \\
\hline$R e_{\max }=\sqrt{\frac{g A_{t}}{6}} \frac{\left(2 h_{m}\right)^{3 / 2}}{v_{\text {mix }}}$ & $\sim 2400$ & $\sim 20000$ \\
\hline$U_{\mathrm{m}}$ & $0.05 \mathrm{~m} / \mathrm{s}$ & $2.0 \mathrm{~m} / \mathrm{s}$ \\
\hline Diagnostics & $\begin{array}{c}\text { Imaging, Thermocouple, } \\
\text { PIV, PLIF }\end{array}$ & $\begin{array}{l}\text { Imaging, Hot-wire } \\
\text { anemometry, Cold-wire } \\
\text { anemometry }\end{array}$ \\
\hline Cost of run & $\sim \$ 0$ & $\$ 33$ per bottle \\
\hline
\end{tabular}


layer mixing is parabolic in the stream-wise direction if the spread angle (of mixing) was small, i.e. $\tan ^{2} \theta<<1$, where $\theta$ was the spread angle. This assumption also applies to the buoyancy driven mixing layer. Unlike a shear layer which grows linearly, a buoyancy layer grows as a quadratic of the stream-wise direction (see Figure 1.3). The experiment design parameters and operating conditions are defined from the mixing width slope and the mix width $(h)$ from Equation 1.1. Details of the parabolic flow requirement are provided in Appendix A. Over the last 12 years, various diagnostics have been established at the water channel facility to extensively study R-T mixing. Diagnostics involve digital image analysis (Snider 1994; Snider \& Andrews 1994; 1995; Snider \& Andrews 1996), particle image velocimetry (Mueschke 2004; Ramaprabhu \& Andrews 2003; 2004; Wilson 2002) and high resolution thermocouple measurements (Mueschke 2004; Mueschke \& Andrews 2005; Ramaprabhu \& Andrews 2003; 2004; Wilson 2002; Wilson \& Andrews 2002). Since the water channel uses hot and cold water, the Atwood number that can be studied is small $\left(A_{t} \sim 10^{-4}-10^{-3}\right)$. However, R-T mixing in ICF occurs at high Atwood numbers and thus high Atwood number buoyancy driven mixing facility was envisioned. This led to the design and construction of the gas channel facility.

The newly designed gas channel facility (see Figure 1.4) is similar in design to the water channel facility. Table 1.2 gives a comparison of the design parameters between the water channel and the gas channel. The channel dimensions are doubled while the aspect ratio has been kept the same. Two gas streams are employed, one of air (top) and the other of a helium-air mixture (bottom). As with the water channel, the two gas 


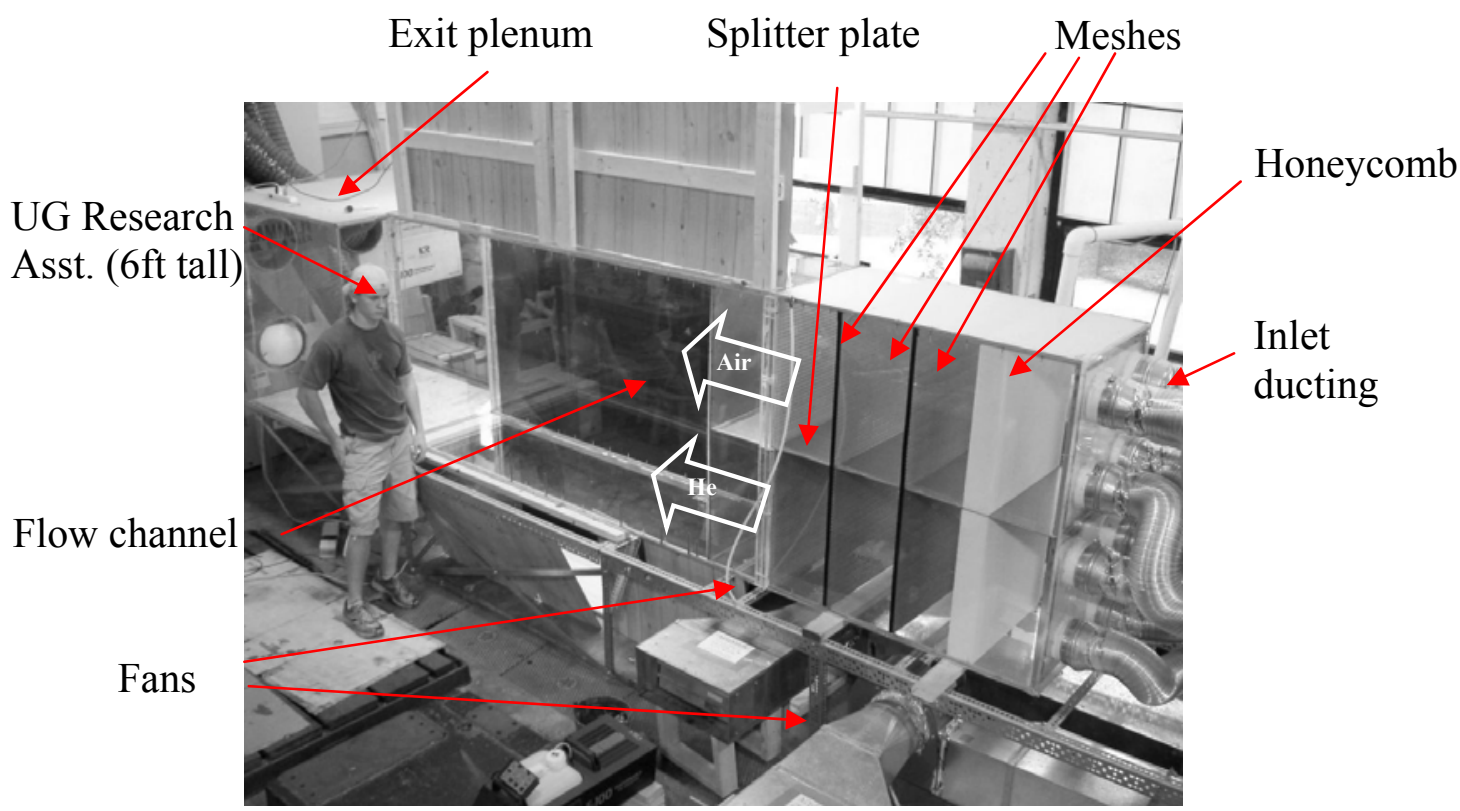

Figure 1.4 Photograph of gas channel facility at Texas A\&M University. 


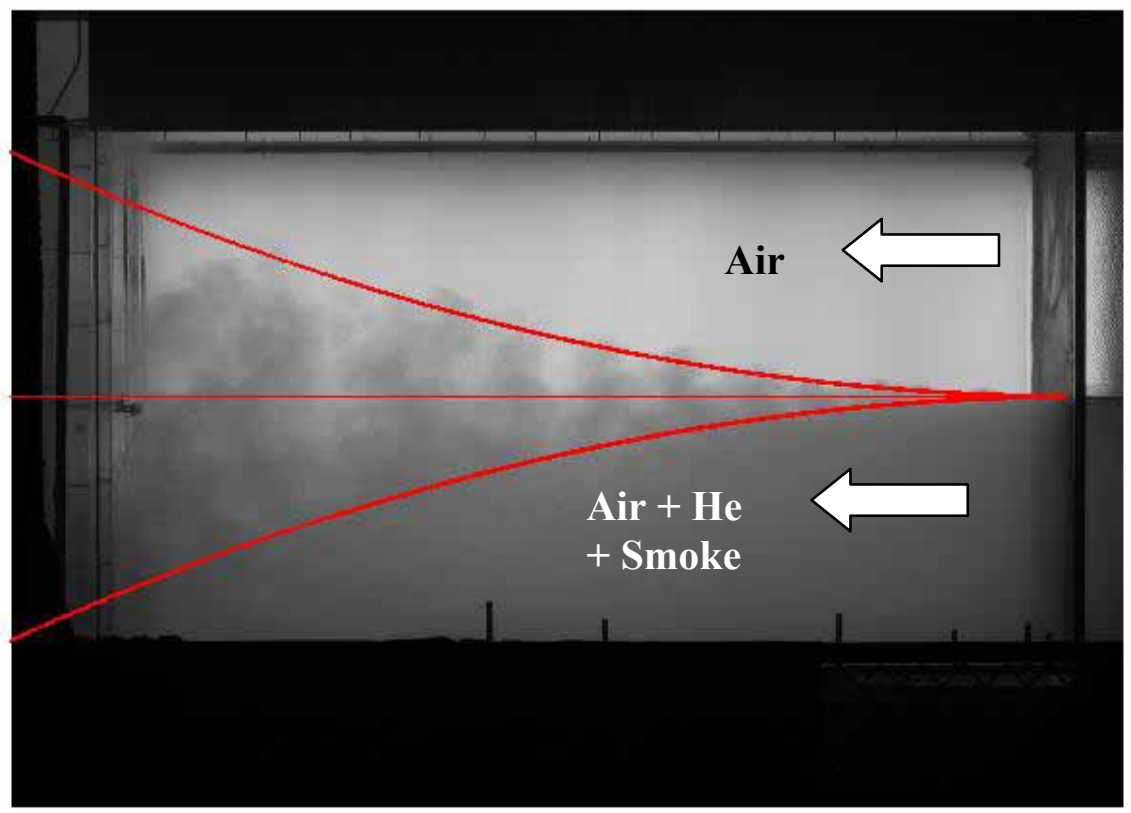

Figure 1.5 Gas channel images of evolution of the R-T instability for $A_{\mathrm{t}} \# 0.04$ $\left(U_{\mathrm{m}}=0.5 \mathrm{~m} / \mathrm{s}\right)$. A parabolic profile was fitted on mixing width $\left(\alpha_{\mathrm{b}}=0.07\right)$. Green smoke was added to the bottom stream for visualization purpose. 
streams flow parallel to each separated by a thin splitter plate. The streams meet at the end of the splitter plate leading to the formation of an unstable interface and of buoyancy driven mixing (see Figure 1.5). The Atwood number is varied by controlling the proportion of helium in the bottom stream. This air-helium buoyancy driven mixing experiment provides significantly larger Reynolds number flows (calculated based on formulation of balance of TKE and PE by Snider and Andrews 1994). The facility provides long data collection times, short transients, is statistically steady in time and is capable of large Atwood number $\left(A_{\mathrm{t}} \leq 0.75\right)$ studies (Banerjee \& Andrews 2006).

\subsection{Hot-Wire Anemometry - Advantages and Limitations}

In the present work, hot-wire anemometry (HWA) is used to investigate R-T mixing. Prior to choosing HWA for measurements, various diagnostics (PIV, LDV and HWA) to measure velocity and density fluctuations were examined. A comparison of the various diagnostics was studied with respect to the requirements for the flow: i.e. probe volume, directional resolution, frequency response, noise level, cost and set-up time for a system. Our findings are tabulated in Table 1.3. The gas channel was built with an aim to obtain high order velocity, density correlations on a time-average and instantaneous basis for a wide range of Atwood numbers. It was seen that both HWA and LDV were feasible diagnostics. Thus a choice was made between the two. In theory, thermal anemometers can be used in almost any fluid-flow situation. However, sensor fragility, calibration shifts due to sensor contamination or difficulty in separating correlated variables may make usage difficult. However, with careful calibration, HWA can be used to get good measurements in a wide variety of situations (Goldstein 1996). In contrast, LDV is 
TABLE 1.3 Comparisons between Hot-Wire Anemometry (HWA), Particle Image Velocimetry (PIV) and laser Doppler Velocimetry (LDV) on the basis of the requirements in the gas channel facility

\begin{tabular}{|c|c|c|c|}
\hline Requirement & HWA & PIV & LDV \\
\hline Probe volume & $5 \mu \mathrm{m} \times 1.25 \mathrm{~mm}$ & $5 \mu \mathrm{m} \times 5 \mu \mathrm{m}$ & $5 \mu \mathrm{m} \times 5 \mu \mathrm{m}$ \\
\hline Frequency response & $100 \mathrm{kHz}-1 \mathrm{MHz}$ & $\sim 30 \mathrm{~Hz}$ & $\sim 30-100 \mathrm{kHz}$ \\
\hline Resolution (Noise level) & $1 / 10000$ & $1 / 1000$ & $1 / 1000$ \\
\hline Cost (for 3 D systems) & Low & High & High \\
\hline Set-up time & Short & Moderate & Moderate \\
\hline
\end{tabular}


relatively more difficult to setup, is more expensive, and simultaneous density/velocity measurements are difficult. Thus, HWA was selected for our facility because of the low costs and relatively short time-frame involved in setting up a system which can measure 3D velocity fluctuations. HWA consisting of both Constant Temperature anemometry (CTA) and Constant Current Anemometry has been used in this research to measure various velocity and density fluctuations as well as velocity-density correlations for a wide range of Atwood numbers $\left(0.035<A_{\mathrm{t}}<0.25\right)$ in the mix. These measurements are to our knowledge, the first use of HWA in the R-T community. Using HWA in our facility has several challenges as itemized below:

(a) The flow is one-dimensional in terms of mean flow but has three dimensional velocity fluctuations.

(b) The flow consists of a binary gas mixture of air and helium and thus density fluctuations are present.

(c) The addition of helium in the bottom stream produces a small temperature gradient to the flow. Furthermore, since the Schmidt number $(S c)$ for the flow is $\sim 1$, the air stream was heated to produce a temperature gradient between the two streams and thus use temperature as a fluid marker. Thus, temperature fluctuations are present in the flow in addition to the density fluctuations.

Thus, careful and detailed calibration of the hot-wire probe over a wide range of velocity, mass fraction, and temperature is needed prior to usage in the gas channel. The details of the various hot-wire methods used are provided in Section 4. 


\subsection{Objectives of Present Research}

The research objectives of this work were itemized below:

1. Design and construct the air-helium gas channel facility at Texas A\&M University to study high Atwood number R-T instability. Details about the gas channel design, construction and working are described in Section 2.

2. Validate the operation of the new facility to ensure that it provides data consistent with previous work done at the low Atwood number water channel facility. To achieve this, the gas channel has been run at a low Atwood number of 0.035. Image diagnostics (Banerjee \& Andrews 2006; Snider \& Andrews 1994) were used to obtain various parameters such as mean or average density profiles across the mixing layer and the evolution of the growth constant $\alpha$. These measurements were then compared with the measurements made at the Water Channel facility. This imaging technique is described in details in Section 3.

3. Measure various velocity-density correlations: $\overline{\rho^{\prime 2}}, \overline{\rho^{\prime} u^{\prime}}, \overline{\rho^{\prime} v^{\prime}}, \overline{u^{\prime 2}}, \overline{v^{\prime 2}}$ and $\overline{u^{\prime} v^{\prime}}$. These measurements will be used for validation of predictive turbulent transport models (Andrews 1986; Besnard et al. 1990; 1992; Snider \& Andrews 1996; Steinkamp et al. 1995; Youngs 1989) as mentioned earlier.

4. Formulate heat transfer correlations in low Reynolds number, variable-density flows. The hot-wire has been calibrated over the entire range of air-helium mix at various overheat ratios (wire temperature) and this data has been used to devise heat transfer correlations in low Reynolds number flows. Details about the correlations are given in Section 5. 


\section{EXPERIMENTAL DESIGN *}

\subsection{Experimental Apparatus}

The experimental set-up is similar to the water channel facility (Snider \& Andrews 1994). However, in the present set-up, two gas streams are employed, one of air and the other of a helium-air mixture. As with the water channel, the two streams flow parallel to each other with the air (heavy) above the helium-air mixture (light), separated by a thin splitter plate. The streams meet at the end of the splitter plate leading to the formation of an unstable interface and a buoyancy-driven mixing layer. This air-helium buoyancy driven mixing experiment allows for long data collection times, short transients and was capable of large Atwood number studies $\left(A_{\mathrm{t}} \leq 0.75\right)$. The experiment is statistically steady in time but not in space as the flow field develops downstream. Pure air on top and pure helium at the bottom provides a maximum facility Atwood number of 0.75 . The Atwood number is varied by altering the proportion of helium in the helium-air mix in the bottom stream.

Figure 2.1 shows the experimental set up. The apparatus consists of an inlet and exit plenum connected by a Plexiglas flow channel which serves as the test section. The gas channel is $3.0 \mathrm{~m}$ long, $1.2 \mathrm{~m}$ wide and $0.6 \mathrm{~m}$ deep. The inlet plenum is divided into two sections. Both sections are connected to separate $250 \mathrm{~W}$ brushless blowers (Dayton, Inc.)

\footnotetext{
* Parts of this section including Figures 2.1, 2.2 and 2.3 have been reprinted with permission from Banerjee A, Andrews MJ. 2006. Statistically steady measurements of Rayleigh-Taylor mixing in a gas channel. Phys. Fluids 18:1-13
} 


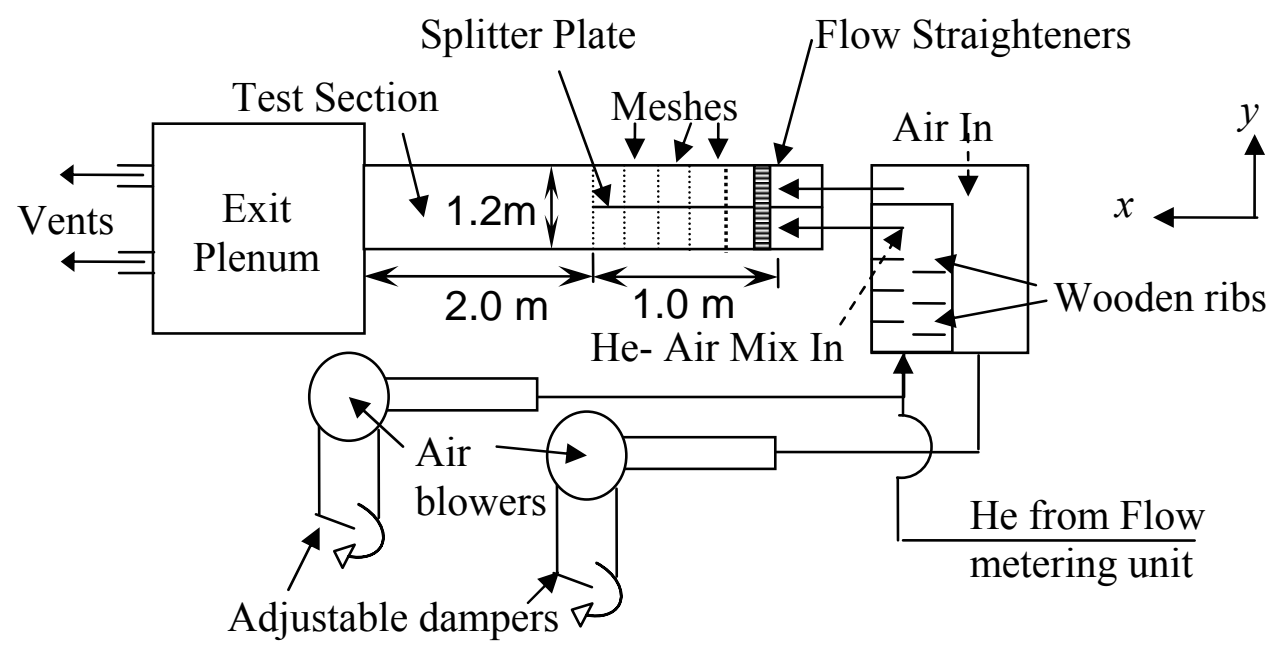

Side View

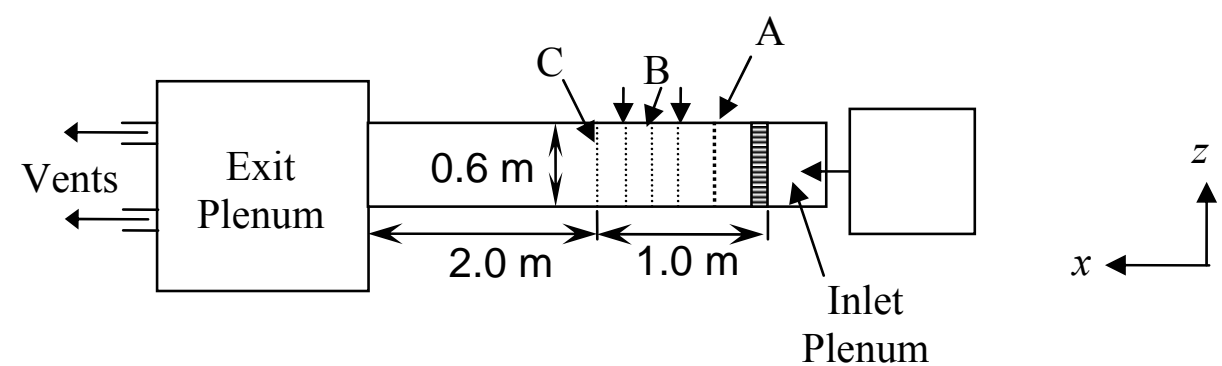

\section{Top View}

Figure 2.1 Schematic of high Atwood number helium-air gas channel facility used for the experiments (Note: for details about wire-meshes A, B and C, see Table 2.1). 
that draw air from the atmosphere. The flow velocity is controlled by adjusting the opening of the dampers connected to the suction port of the blowers. A maximum flow velocity of $2 \mathrm{~m} / \mathrm{s}$ is available (which corresponds to the maximum volume flow rate of the blowers and static pressure drop). In addition, a series of high pressure regulators (TESCOM, Inc., Model \#'s 44-1315-2082-002 and 44-1316-2082-002) and an orifice plate meter helium into the lower section of the inlet plenum. Helium and air streams are uniformly mixed before they reach the inlet section by passing the streams around a series of wooden ribs placed inside the ductwork (see Figure 2.1). A single normal hotwire probe was placed at various locations at the end of the splitter plate to confirm that the mix was uniform. If the mix was not uniform, pockets of helium present in the flow change the heat transfer characteristics of the hot-wire probe leading to a spike in the voltage signal when it flows over the probe. A stainless steel splitter-plate extends from the channel entrance to the start of the test section and separates the two streams of gas. The splitter plate is $0.32 \mathrm{~cm}$ thick, $1.0 \mathrm{~m}$ long and has a $1.8^{\circ} \mathrm{knife}$ edge at the end. The test section after the splitter plate is $2.0 \mathrm{~m}$ long.

The top and bottom inlet sections of the channel are fitted with screens and flow straighteners to produce a uniform flow, assist in dissipating free stream turbulence, and minimize the boundary layer on the splitter plate and walls (Browand \& Weidman 1976; Snider \& Andrews 1994; Stillinger et al. 1983). A $10 \mathrm{~cm}$ long flow straightener sits at the entrance of each channel and is made of polycarbonate honeycomb (Model \# PCFR250W4.00 Plascore, Inc.), in which each honeycomb cavity is $0.635 \mathrm{~cm}$ in diameter. The flow straightener is followed by four sets of screens (Universal Wire 
Cloth, Inc.), one $30 \times 30$ mesh (wire/inch) with a wire diameter of $0.034 \mathrm{~cm}(37.1 \%$ free area) followed by three $30 \times 30$ meshes (wire/inch) with a $0.0216 \mathrm{~cm}$ wire diameter (55.4\% free area). A full channel screen is placed at the end of the splitter plate as it is found to be effective in minimizing the wake from the splitter plate (Koop 1976). This end screen consists of a $22 \times 22$ mesh (wire/inch) with a $0.033 \mathrm{~cm}$ wire diameter and has a free area of $49.8 \%$ (see Table 2.1). The honeycomb and meshes are placed sufficiently upstream to dissipate any free stream turbulence (Tan-Atichat et al. 1982). The free area chosen was consistent with turbulence management recommendations for wind tunnels (Loehrke \& Nagib 1972).

The velocities of the two streams were set so that there was no shear between the flows $\left(U_{\text {air }}=U_{\text {mixture }}=U_{m}\right)$. This was ensured by introducing puffs of smoke in both the top and bottom sections of the channel through small holes on bottom and top and checking for shear. When at no shear, since the cross sectional area $(A)$ of the top and bottom sections was identical, the volumetric flow rate of air and helium- air mixture in the top and bottom channels respectively were equal. The mixture flow rate in the bottom section of the channel was then given by:

$$
\dot{V}_{\text {bottom }}=\dot{V}_{\text {air }}+\dot{V}_{H e}=\dot{V}_{\text {air }}+\frac{\dot{m}_{H e}}{\rho_{H e}}=U_{m} A
$$

The density of the helium-air mixture depends on the mass flow rate of Helium $\left(\dot{m}_{H e}\right)$ and the velocity of the two streams $\left(U_{\mathrm{m}}\right)$ as:

$$
\rho_{\text {mix }}^{\text {bottom }}=\rho_{\text {air }}+\frac{\dot{m}_{H e}}{U_{m} A}\left[1-\frac{\rho_{\text {air }}}{\rho_{H e}}\right]
$$


TABLE 2.1 List of flow-straighteners and meshes in the inlet section of the facility (Note: for the location of each of these screens, see Figure 2.1)

\begin{tabular}{|c|c|c|c|c|}
\hline Mesh Size & Wire Diameter & \% Open Area & Location & Quantity \\
\hline $30 \times 30$ & $0.0340 \mathrm{~cm}$ & $37.1 \%$ & $\mathrm{~A}$ & 1 \\
\hline $30 \times 30$ & $0.0216 \mathrm{~cm}$ & $55.4 \%$ & $\mathrm{~B}$ & 3 \\
\hline $22 \times 22$ & $0.0330 \mathrm{~cm}$ & $49.8 \%$ & $\mathrm{C}$ & 1 \\
\hline
\end{tabular}


The Atwood Number of the mix was hence given by:

$$
A_{t}=\frac{\left(\rho_{\text {air }}-\rho_{\text {mix }}^{\text {bottom }}\right)}{\left(\rho_{\text {air }}+\rho_{\text {mix }}^{\text {bottom }}\right)}=\frac{\left[\frac{\rho_{\text {air }}}{\rho_{H e}}-1\right] \frac{\dot{m}_{H e}}{U_{m} A}}{2 \rho_{\text {air }}+\left[1-\frac{\rho_{\text {air }}}{\rho_{H e}}\right] \frac{\dot{m}_{H e}}{U_{m} A}}
$$

Since the mass flow rate of helium $\left(\dot{m}_{H e}\right)$ was needed to evaluate the Atwood number in Equation 2.3, an accurate measurement of the helium mass flow rate was required. Furthermore, $\rho_{\text {air }}$ and $\rho_{H e}$ were dependent on the fluid temperatures. Thus, to get an accurate estimate of the Atwood number, the densities of air and helium were obtained from equations of state for the gases (see Appendix C).

\subsection{Mass Flow Rate Calibration}

Initial consideration was given to using a commercial gas flow meter or controller. However, for the range of pressures $(\sim 2100 \mathrm{psig})$ and mass-flow rates being used $(\sim 0.1$ $\mathrm{lbm} / \mathrm{s}$ ), such flow meters are expensive and complex. Furthermore, calibration data obtained from the manufacturers are based on air and use of empirical laws to compensate for the effects of Helium meant that the flow meters would require recalibration. Thus, it was decided to use a volumetric method at constant outlet pressure for flow metering, in which, the gas was delivered from a supply, having passed through an orifice (Jitschin et al. 1995). The main feature of the current set-up is the use of a thin orifice for flow constriction and metering. The pressure drop across the orifice is maintained so that the pressure ratio between the downstream and upstream locations is below the critical pressure ratio. Hence, the flow is choked at the orifice and thus, 


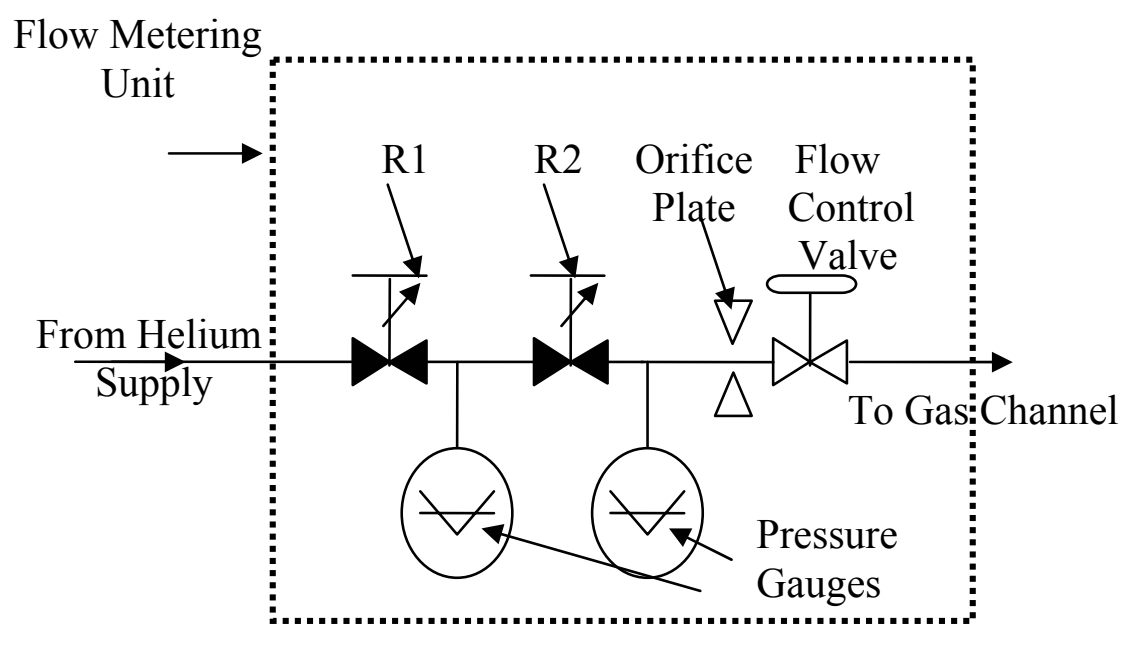

Figure 2.2 Schematic of flow metering unit for a constant mass flow rate of helium (R1: Regulator 1: 0-1500 psig; R2: Regulator 2: 0-1000 psig). 


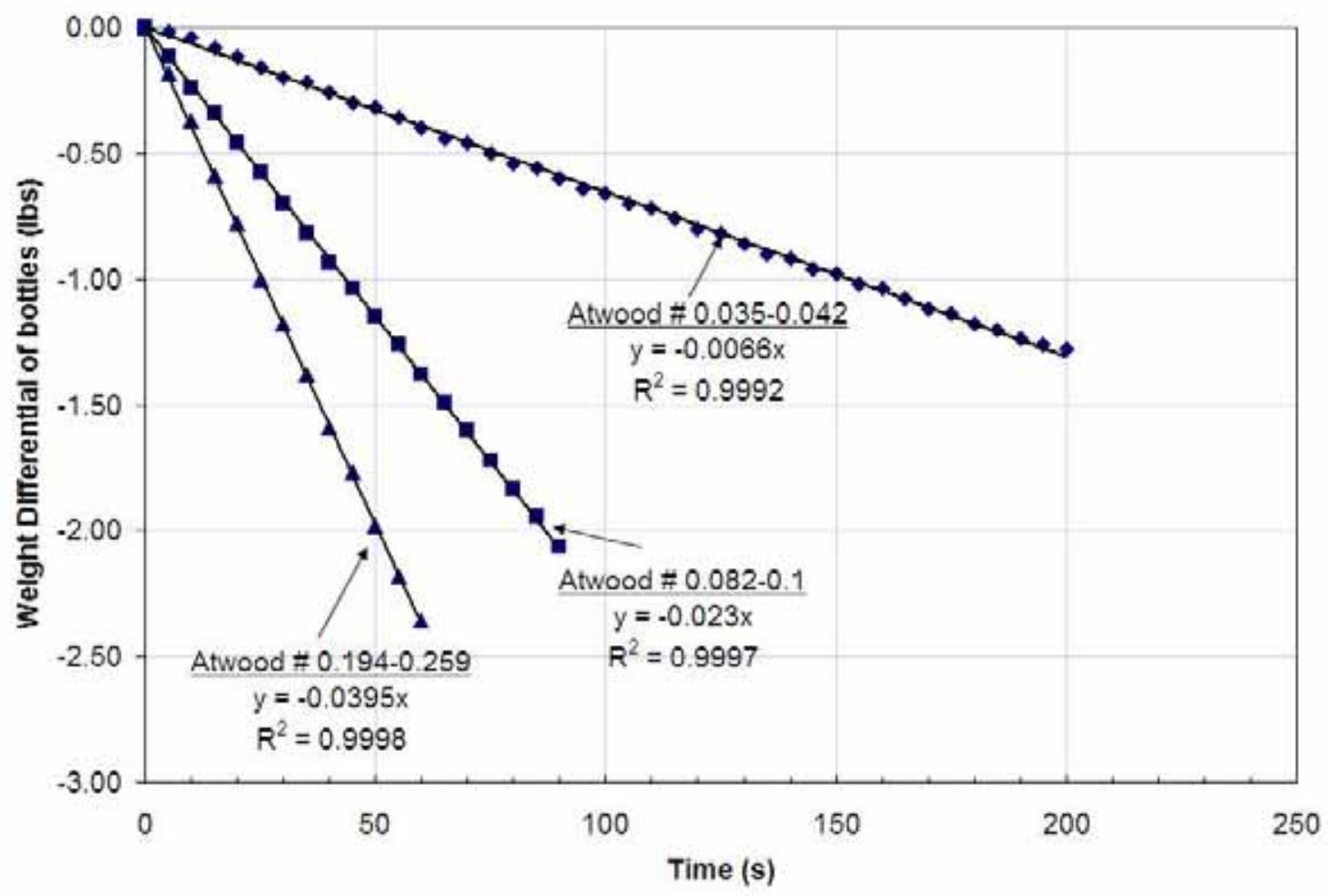

Figure 2.3 Calibration of mass flow rate of helium for different Atwood numbers. The measurement uncertainty of digital scale is $\pm 0.01 \mathrm{lbs}$. (Note: For the mass flow rate calibration corresponding to an Atwood \# 0.25, 2 bottles of helium were used for calibration. So the actual mass flow rate was twice the slope.) 
TABLE 2.2 Calibrated mass flow rates for different orifice (Note: The measurement uncertainty of digital scale is $\pm 0.01 \mathrm{lbs}$. For the uncertainty in the measured Atwood number, see Appendix B.3)

\begin{tabular}{|c|c|c|c|c|}
\hline \multirow{3}{*}{ No. of units } & Diameter of & \multicolumn{2}{|c|}{ Mass Flow Rate $(\mathbf{l b m} / \mathbf{s})$} & \multirow{2}{*}{$\begin{array}{c}\text { Atwood } \\
\text { Orifice (inch) }\end{array}$} \\
\cline { 3 - 4 } & Eumber \\
\hline \multirow{3}{*}{1} & 0.032 & $0.0066 \pm 0.00007$ & 0.0072 & $\sim 0.04$ \\
\cline { 2 - 4 } & 0.061 & $0.0234 \pm 0.00007$ & 0.0267 & $\sim 0.10$ \\
\hline \multirow{3}{*}{2} & 0.072 & $0.0284 \pm 0.00014$ & 0.0339 & $\sim 0.22$ \\
\cline { 2 - 4 } & 0.110 & $0.0507 \pm 0.00014$ & 0.0793 & $\sim 0.31$ \\
\cline { 2 - 4 } & 0.150 & $0.0654 \pm 0.00014$ & 0.1437 & $\sim 0.38$ \\
\cline { 2 - 4 } & 0.180 & $0.0825 \pm 0.00014$ & 0.2365 & $\sim 0.47$ \\
\cline { 2 - 4 } & 0.225 & $0.1350 \pm 0.00014$ & 0.3316 & $\sim 0.72$ \\
\hline
\end{tabular}

1 At this orifice size, the flow rates were extremely high. These flow rates were near the limit that the TESCOM regulators can handle. It was recommended to branch the Helium metering system to at least 3 units so that the effective flow rate through each regulator was reduced. 
the mass flow rate through it was determined based on empirical relations $(\mathrm{Wu} \&$ Molinas 2001). To this end, the mass flow rate was measured by placing helium bottle(s) on a sensitive digital scale $( \pm 0.01 \mathrm{lbs})$ and recording the change in weight of the bottle with time.

Figure 2.2 shows a schematic of the setup used for controlling and metering the mass flow rate of helium into the gas channel. Two high pressure regulators, $R 1$ and $R 2$ (TESCOM, Inc.), are used to control the pressure drop from a supply pressure of $\sim 2100$ psig to the ambient pressure inside the channel. Two high pressure gauges (Swagelock, Inc.) are connected as shown in the schematic to accurately read the pressure in the line at two different downstream locations to ensure that the flow chokes at the orifice and not at either of the regulators. Flexible $1 / 2$ " diameter stainless steel tubing is used to connect all components. An orifice plate is placed after the downstream pressure gauge and held in position by the flow control valve. Initially, the flow control valve is closed and the pressure regulators are adjusted: the upstream regulator is fixed at $1050 \mathrm{psig}$ to ensure that the pressure ratio across $R l$ exceeds the critical pressure ratio and thus the flow is not choked at $R 1$; and the downstream pressure regulator $R 2$ is set at $550 \mathrm{psig}$ to ensure the flow is not choked at $R 2$. Thus, when the flow control valve is opened, the flow is immediately choked at the orifice and a constant mass flow rate of helium until the pressure in the bottles dropped below the set pressure (550 psig in this case). Table 2.2 shows the results of the mass flow rate calibrations for different orifices of diameters. The theoretical mass flow rate was calculated based on equations for sub-critical flow through the orifice (John 1984). 
Figure 2.3 shows the mass flow rates of helium measured on the digital scale with three different orifices. A straight line fit was performed through the data points obtained and the mass flow rates were tabulated. The $R^{2}$ values for the fit in these cases vary between 0.9992 and 0.9998. The Atwood number range given in Table 2.2 was calculated based on the experimental mass flow rate for a given mean velocity (to achieve the parabolic approximation). This facility arrangement empties $90 \%$ of the Helium tanks at constant mass flow rate before the threshold of 550 psig is reached. However, for the higher mass flow rates required for the high Atwood runs $\left(A_{\mathrm{t}} \geq 0.5\right)$, the flow rates exceed the maximum flow rates that can be accommodated by the regulators. Hence, two helium metering units were connected in parallel to accommodate the higher flow rate demands. Thus for $A_{\mathrm{t}} \geq 0.25$, both the systems are used in parallel to give larger run times for the experiment. A Kline McClintock uncertainty analysis (1953) was performed to calculate the uncertainty in the Atwood number for the experiment and is provided in Appendix B. The Atwood number range tabulated below was calculated based on the experimental mass flow rate and varying the angle of spread in the mix between $10^{\circ}-15^{\circ}$. 


\section{VISUALIZATION DIAGNOSTICS *}

\subsection{Visualization Technique - Calibration}

The lighter fluid (air-helium mixture at the bottom) was colored with dark green smoke (RC105G, Regin HVAC Products) to visualize the mixing layer. A row of 35 fluorescent lamps backlit the entire channel test section while Matte (frosted) Acetate paper (MisterArt.com) serves as the white background and helped diffuse the light. Each experiment was photographed using a Canon Powershot A80 digital camera. The digital camera stored the pictures in JPEG format. Pictures were captured continuously at the rate of 80 images per minute. The camera settings were manually chosen to eliminate variations between images. Before the experiment was run, the shutter speed, aperture and ISO settings were set so that it did not change during a run. The values used for different Atwood number runs were given in Table 3.1. A manual focus was also used so that the camera did not try to auto-focus on moving structures during the experiment. The images were then cropped at the same location using a marker near the exit plenum so that the mix width spanned the entire width of the image. The images were processed and analyzed using MATLAB ${ }^{\odot}$. The relation between concentration and pixel intensity was determined by using a calibration wedge. A calibration wedge is a triangular Plexiglass container filled with the same green smoke. The wedge has a depth (a) of 22 inches, width (b) of 24 inches and height (c) of 6 inches. It was found from a wedge

\footnotetext{
*. Parts of this section including Figures 3.1, 3.2, 3.3, 3.4 and 3.5 have been reprinted with permission from Banerjee A, Andrews MJ. 2006. Statistically steady measurements of Rayleigh-Taylor mixing in a gas channel. Phys. Fluids 18:1-13
} 
TABLE 3.1 Camera (Canon Powershot A80) settings at different Atwood numbers (Note: For measurement uncertainties of Atwood number and mean velocity, see Appendix B).

\begin{tabular}{|c|c|c|c|c|}
\hline Atwood Number & $\boldsymbol{U}_{\mathrm{m}}(\mathbf{m} / \mathbf{s})$ & Shutter Speed & Aperture & ISO \\
\hline 0.035 & 0.6 & $1 / 100 \mathrm{~s}$ & $\mathrm{~F} / 8.0$ & 50 \\
\hline 0.259 & 1.2 & $1 / 100 \mathrm{~s}$ & $\mathrm{~F} / 8.0$ & 100 \\
\hline
\end{tabular}




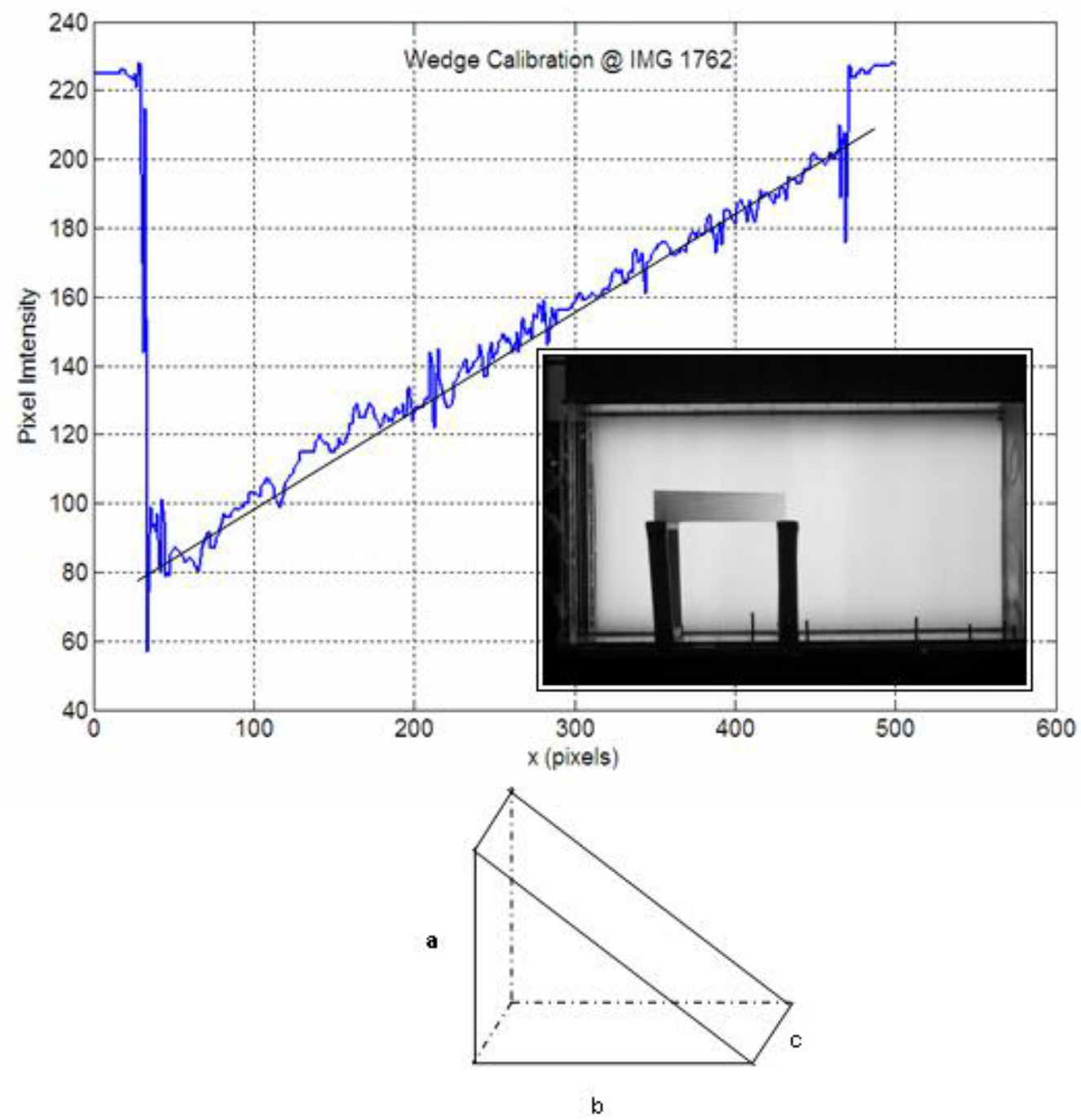

Figure 3.1 Intensity as a function of height for calibration wedge (Inset shows actual calibration image) [wedge dimensions: $\mathrm{a}($ depth $)=22$ inches, $\mathrm{b}$ (width) $=24$ inches, $\mathrm{c}$ (height) $=6$ inches) $]$. 
calibration (Banerjee \& Andrews 2006; Snider \& Andrews 1994) that the concentration of smoke must be kept low to maintain a linear relation between the concentration and measured intensity. Figure 3.1 shows that the camera response was linear for a dynamic range over 100 pixel intensity values. For grayscale values less that 80 (lower means darker), it was found the camera response became non-linear. Thus, care was taken to ensure that the calibrated linear dynamic range from 100 to 200 was used during an experimental run.

\subsection{Correction for Non-Uniform Backlight}

Extinction of light from source $I_{\mathrm{o}}$ across the path $z$ is given by Beer Lambert's Law, where $\kappa$ is the monochromatic extinction coefficient (see Equation 3.1). Expanding the exponential in a series and retaining the first term gives a linear relationship between the applied and transmitted intensities along path $z$.

$$
I_{m}(x, y)=I_{0} \exp \left(-\int_{0}^{z} \kappa d z\right) \approx I_{0}\left[1-\int_{0}^{z} \kappa d z\right]=I_{0}(1-\omega)
$$

where, $\omega=\int_{0}^{z} \kappa d z$ is the absolute extinction coefficient of the medium. For this experiment, the extinction coefficient is a function of the volumetric concentration of smoke and the optical path length of light traveled. The calibration (Figure 3.1) shows that this approximation is valid from $0-60 \%$ extinction of the light.

In an ideal experiment, the test section is irradiated with a uniform backlight, and then any two points in the image with the same intensity is a result of an identical attenuation of light. Using a linear relationship between the smoke concentration and 
intensity, the concentration of the flow field can be defined from the intensity distribution. The images of the channel during the run were found to be bright at the center of the photograph and darker towards the edges. On first look, this effect may be misinterpreted as optical vignetting. A photograph or drawing whose edges gradually fade into the surrounding paper is called a vignette (Smith 2000). Most lenses exhibit optical vignetting to some degree. The effect is strongest when the lens is used at wide open apertures. Thus, the effect of vignetting was avoided by using a smaller aperture (see Table 3.1). The gradual darkening of the edges was an effect on the non-uniform light intensity of the fluorescent tubes which were bright at the center and darker towards the edges. So, a background image (see Figure 3.2) was used to correct for nonuniformities in the background intensity to a uniform intensity.

The background image was taken from a photograph of the test section without the smoke. The present work used the method of Snider and Andrews $(1994,1995)$ for correcting the linear intensity to extinction approximation. The corrected measured intensity $I_{\text {corr }}$ that would exist if the background intensity was uniform ( $I_{o}^{\text {uniform }}$ ) can be written as:

$$
I_{\text {corr }}=I_{0}^{\text {uniform }}(1-\omega)
$$

Since $\omega$ is a function of the depth of the channel, it can be determined from the measured intensity $I_{\mathrm{m}}$ and the background intensity $I_{0}$. Thus, eliminating $\omega$ between Equations 3.1 and 3.2 gives:

$$
I_{\text {corr }}=\frac{I_{0}^{\text {uniform }}}{I_{0}} I_{m}
$$




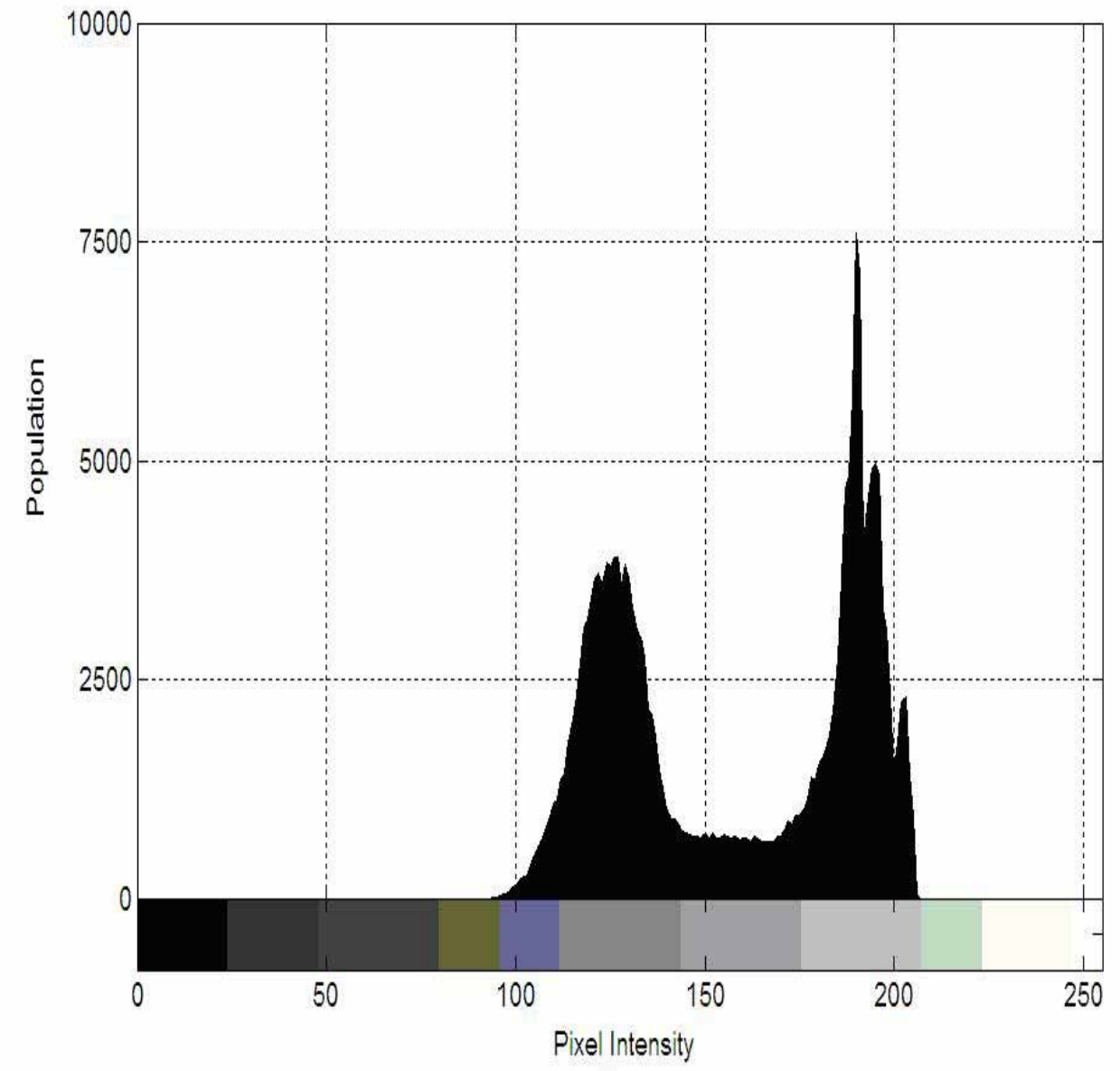

Figure 3.2 Histogram for raw image. 


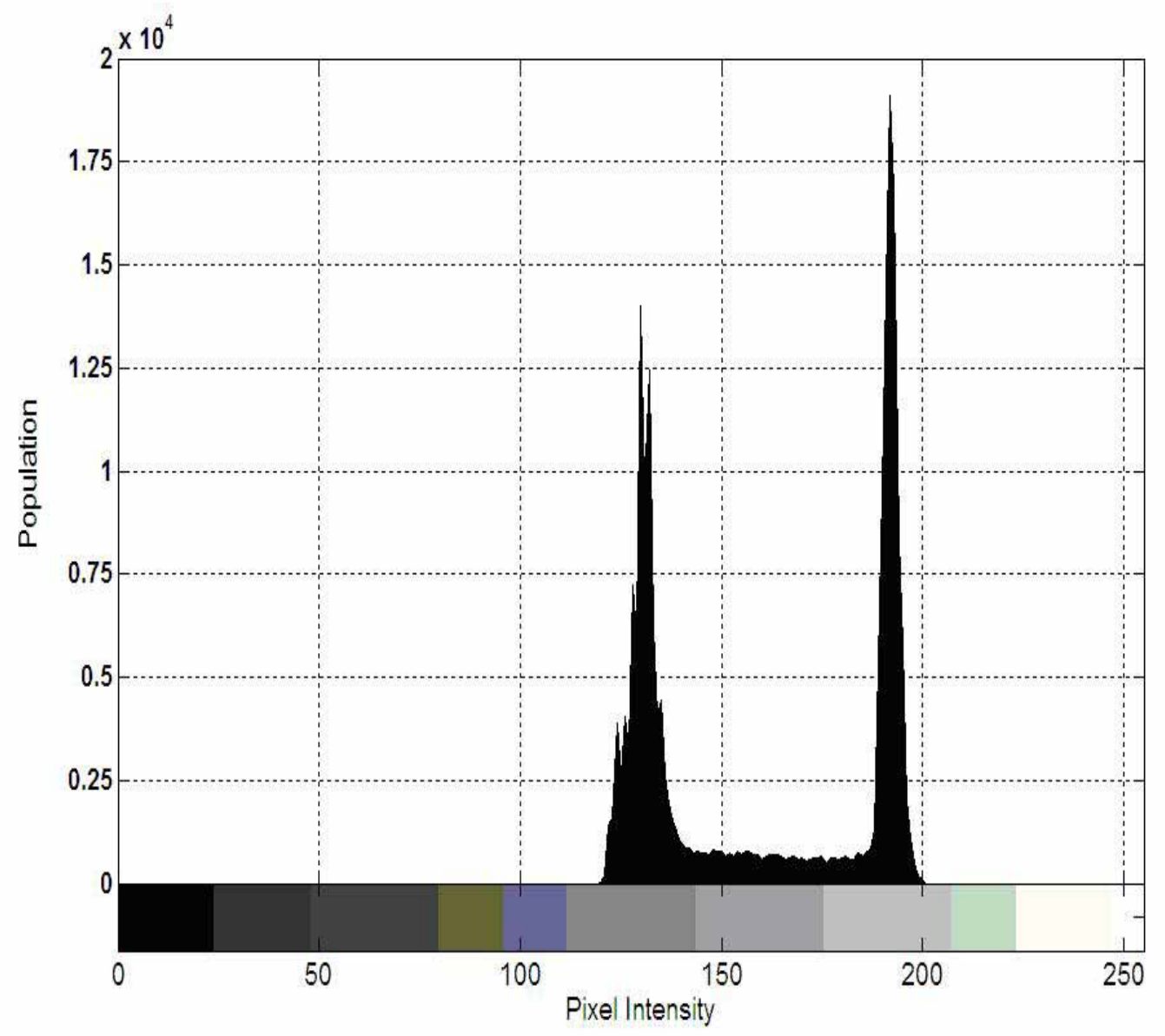

Figure 3.3 Histogram for processed image. 
The corrected intensity, $I_{\text {corr, }}$ is the intensity that would exist if the backlighting is uniform and takes into account the actual background intensity and the measured intensity for each pixel. The peak value of a histogram of the background image was selected as $I_{0}^{\text {uniform }}$. Figures 3.2 and 3.3 shows the intensity population before and after adjusting for non-uniform backlighting. In Figure 3.2, the peaks correspond to regions at the edge or outside the mix, and were thus more prone to non-uniformity in backlighting. The correction for non-uniform backlighting gives narrower peaks for pixel populations at low and high intensities, which correspond to regions where the concentrations were $0 \%$ and $100 \%$. This shows the improvement obtained with the backlighting correction, as these were the regions at the periphery of the mix region where the non-uniformity in backlighting was more pronounced.

\subsection{Steps in Image Processing Diagnostics}

The following steps were followed to capture images of the channel before and during an experimental run:

- Fix digital camera settings manually (refer to Table 3.1). Check image histogram to ensure that the peaks were within the linear range of calibration.

- Take background image before and after completion of run.

- Run channel and take pictures in "continuous-burst" mode.

Once the run was completed, the images were transferred from the camera memory to a PC. To process the background images, the following steps were adopted:

- Process the background images and store an averaged background image. 
- Locate 3 physical points on the averaged background image and crop image. If the image was tilted, rotate background image. Pick an origin in the image. Pick 2 locations on the image of known distance to calculate physical distance per pixel.

- Check histogram of background image and find the pixel intensity value corresponding to the peak of the histogram. Denote this value as $I_{o}^{\text {uniform }}$ in the background correction (see Equation 3.3).

- Store processed background image.

Once the background Image has been processed and stored, the images taken during the run were first converted to grayscale and then processed as follows:

- Average all images during the run. Locate 3 physical points on the averaged image and crop image. If the image was tilted, rotate the image.

- Check histogram of averaged image.

- Perform background correction based on Equation 3.3.

- Check histogram of corrected image.

- Store processed average image.

This averaged image can be used for drawing fraction profiles; image contours as well as computing the mix widths and the growth constant $\alpha$ for the experimental run (see Section 6). 


\subsection{Qualitative Measurements}

Figures $3.4 \mathrm{a}-\mathrm{b}$ show photographs of experimental runs at Atwood numbers of 0.04 $\left(U_{\mathrm{m}}=0.5 \mathrm{~m} / \mathrm{s}\right)$ and $0.097\left(U_{\mathrm{m}}=0.85 \mathrm{~m} / \mathrm{s}\right)$. Two gas streams, one containing pure air (top half) and the other consisting of air-helium mixture colored with green smoke (bottom half), flow parallel to the splitter plate. The photograph shows the formation and evolution of buoyancy driven mixing layer. As described previously, care was taken to ensure that there was no shear between the two streams. Figures 3.2 and 3.3 show a lack of fluctuations or variation of smoke outside the mixing layer. This indicates that large scale plumes and small disturbances were confined to the mixing layer. This behavior was common in plane shear flow where turbulence was associated with shearing of the stream-wise flow (Brown \& Roshko 1974; Tennekes \& Lumley 1994) and was also apparent in the present buoyancy driven flow. The mixing process observed is one of engulfment of air-helium from below and air from above. This resulted in a mix region, whose height increased with downstream distance. Figures $3.5 \mathrm{a}-\mathrm{b}$, are close up views of the mix, and show that that the buoyant mixing layer grows as a front of rising and falling plumes ("bubble and spikes"). Multiple plumes form span-wise across the channel. Vortices form around the edge of the mushroom head of light or heavy fluid as they penetrate through each other. Four major structures are observed at regular frequency along the stream-wise direction (right to left). The last two structures in the series (extreme left) are seen to pair. The pictures show that an advanced plume (extreme left) pairs with an earlier plume (second from left). Such "bubble competition" is observed throughout the length of the mix. 


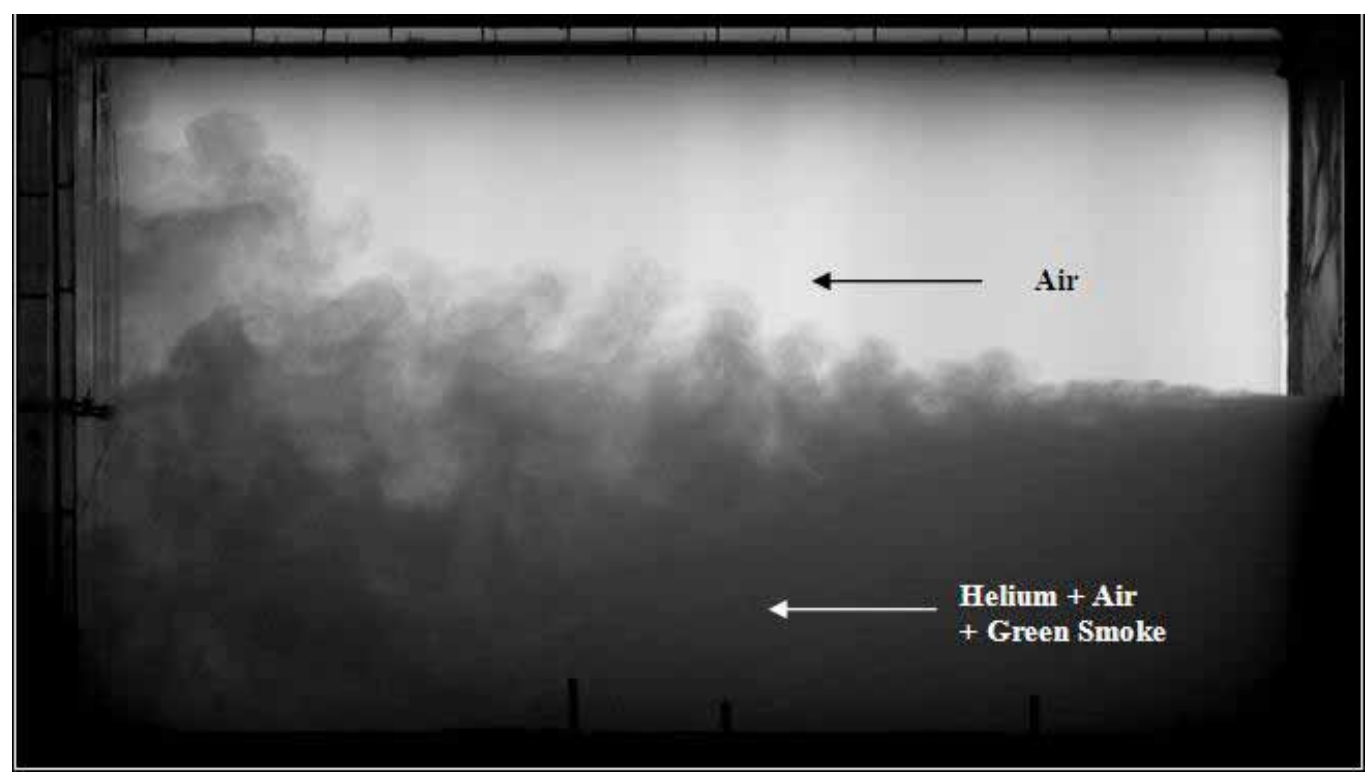

(a)

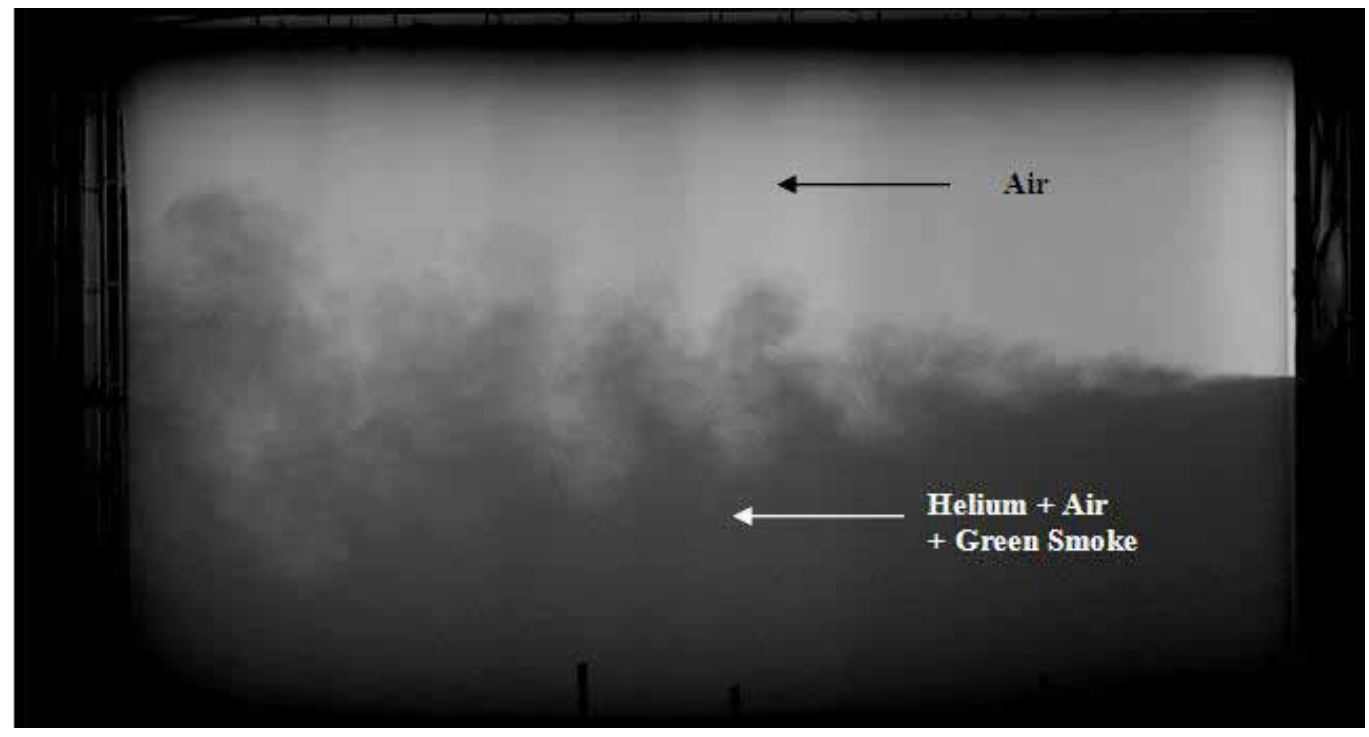

(b)

Figure 3.4 View of the mixing process in the channel at (a) $A_{\mathrm{t}} \# 0.04\left(U_{\mathrm{m}}=50 \mathrm{~cm} / \mathrm{s}\right)$ and (b) $A_{\mathrm{t}} \# 0.097\left(U_{\mathrm{m}}=85 \mathrm{~cm} / \mathrm{s}\right)$. 


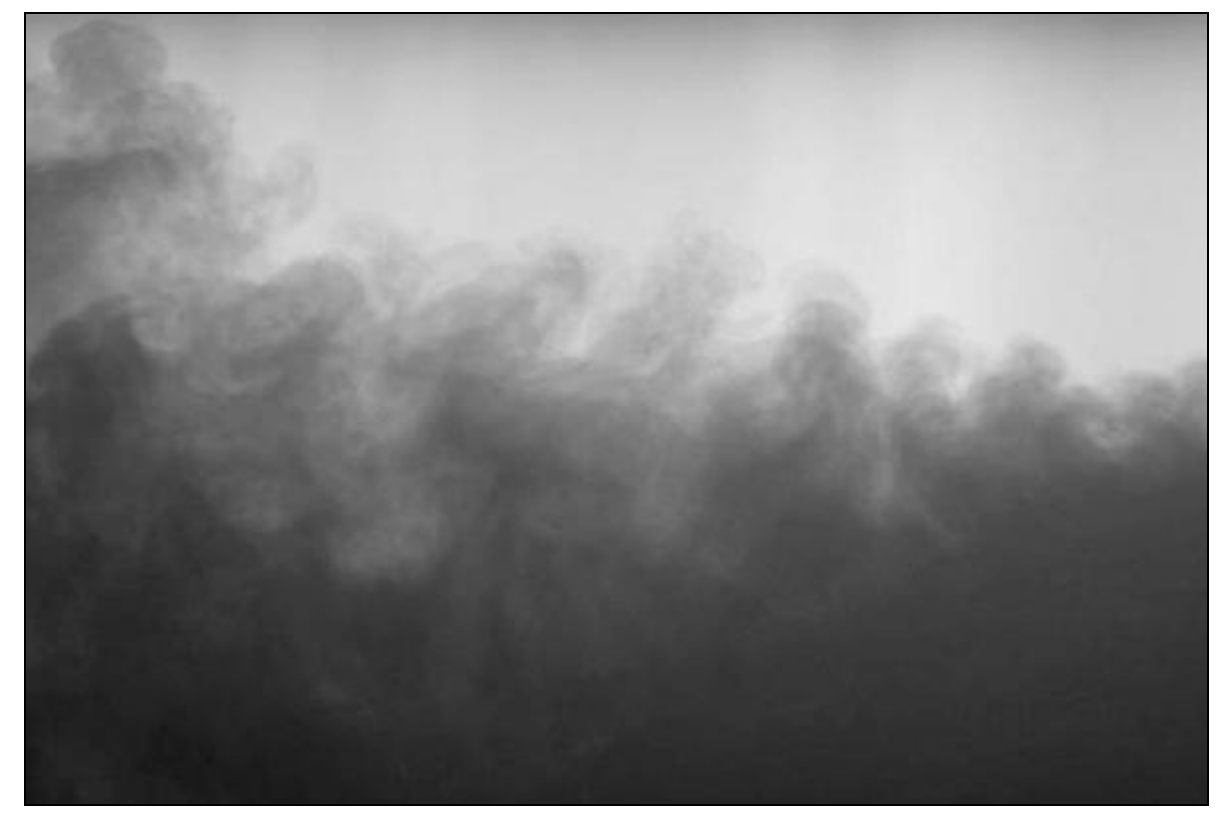

(a)

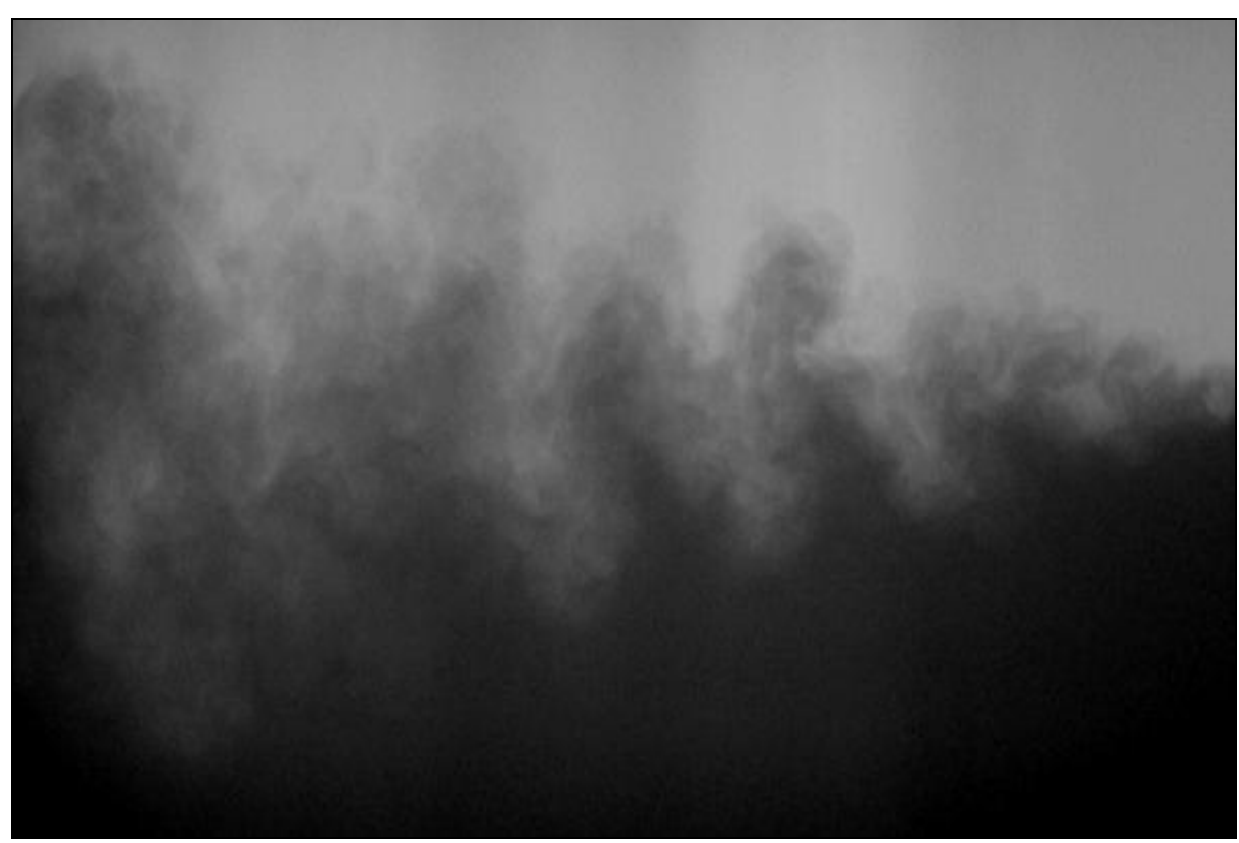

(b)

Figure 3.5 Close-up view of the three dimensional plumes across the channel for (a) $A_{\mathrm{t}} \#$ $0.04\left(U_{\mathrm{m}}=50 \mathrm{~cm} / \mathrm{s}\right)$ and $(\mathrm{b}) A_{\mathrm{t}} \# 0.097\left(U_{\mathrm{m}}=85 \mathrm{~cm} / \mathrm{s}\right)$. 


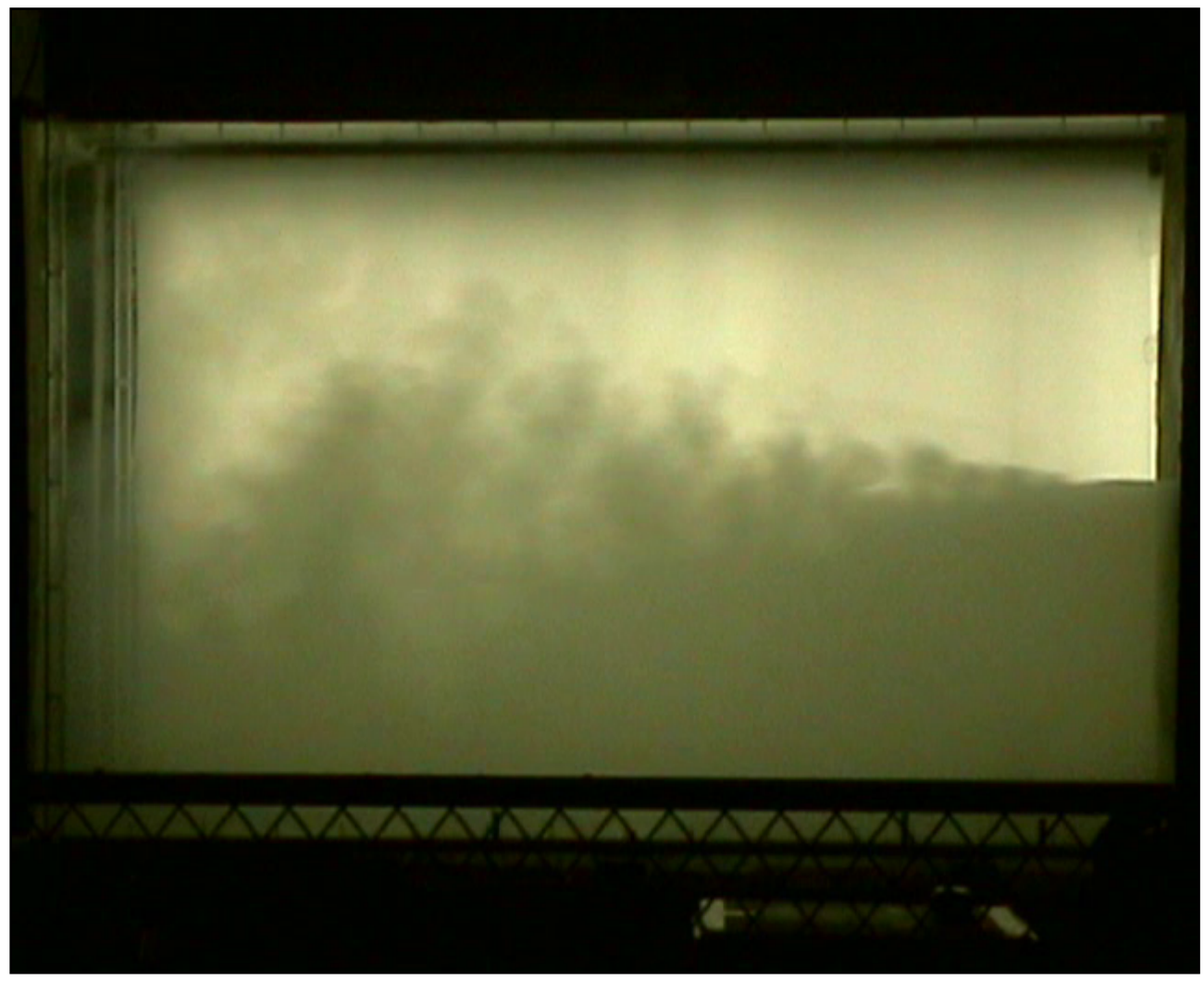

Figure 3.6 Movie of the mixing process for $A_{\mathrm{t}} \# 0.259\left(U_{\mathrm{m}}=1.2 \mathrm{~m} / \mathrm{s}\right)$. 


\section{HOT-WIRE DIAGNOSTICS}

Velocity measurements were made using a hot-wire Probe of diameter $5 \mu \mathrm{m}$ (single wire: 55P16) coupled to a Constant Temperature Anemometer (CTA) unit (MiniCTA, Dantec Dynamics). Temperature measurements were made using the Single Normal (SN) wire probes (55P16) as cold wire probes coupled to a Constant Current Anemometer unit (A.A. Laboratories, Inc.). Although our use of CTA and CCA was typical, we briefly review its application to measurement of our R-T mix, and note refinements and differences from the typical set up.

\subsection{Constant Temperature Anemometry (CTA) for Measuring Velocity Fluctuations}

The CTA unit is an analog instrument designed for measuring velocity in gases and works on the basis of convective heat transfer from a heated sensor to the surrounding fluid, the heat transfer being primarily related to the fluid velocity. The CTA is suited for measurement of fast gas velocity fluctuations of fine scales and high frequencies (Jørgensen 1996). The complete measuring system used in the present study consists of (a) Single Normal Hot-wire probe (SN probe: 55P16) with support and 4-m BNC-BNC probe cable, (b) Mini-CTA anemometer (54T30) with built-in signal conditioner and power adapter, (c) SC 2040 Sample and Hold Board (National Instruments) connected to a PCI-MIO-16E-4 A/D board (National Instruments) mounted in a Pentium 4 computer. NI-DAQ driver software and Lab-View DAQ Software are used for data collection at 


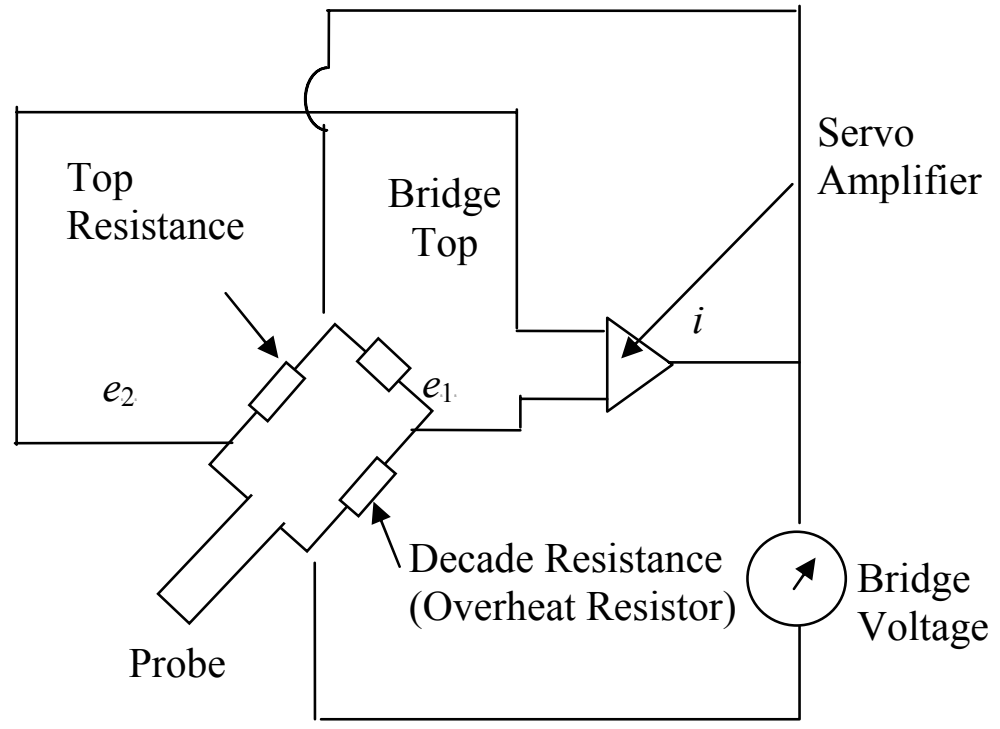

Figure 4.1 The constant temperature anemometer (CTA) circuit. 
TABLE 4.1 Sample operating parameters for SN wire coupled to a mini-CTA unit $($ overheat ratio $=1.6)$

\begin{tabular}{|l|c|c||}
\hline \multicolumn{3}{|l|}{ Probe specific parameters. } \\
\hline Sensor resistance, $R_{20}$ & 3.49 & $\Omega$ \\
\hline Sensor lead resist., $R_{\mathrm{L}}$ & 0.9 & $\Omega$ \\
\hline Support resistance, $R_{\mathrm{s}}$ & 0.44 & $\Omega$ \\
\hline Cable resistance, $R_{\mathrm{c}}$ & 0.2 & $\Omega$ \\
\hline Sensor TCR, $\alpha_{20}$ & 0.0036 & $/ \mathrm{K}$ \\
\hline Wire operating parameters & \\
\hline Desired wire temp., $T_{\mathrm{w}}$ & 191.43 & ${ }^{\circ} \mathrm{C}$ \\
\hline Operating resistance, $R_{\mathrm{w} .}$ & 5.64 & $\Omega$ \\
\hline Total resistance, $R_{\mathrm{T}}$ & 7.18 & $\Omega$ \\
\hline Decade resistance, $R_{\mathrm{D}}$ & 142.48 & $\Omega$ \\
\hline Bridge ratio, $M$ & $1: 20$ & \\
\hline Overheat ratio, a $\left(=R_{\mathrm{w}} / R_{\mathrm{a}}\right)$ & 1.6 & \\
\hline
\end{tabular}


desired frequencies. Figure 4.1 shows the CTA circuit that was used. A hot-wire probe was placed in a Wheatstone bridge. As the flow conditions vary, the error voltage $e_{2}-e_{1}$ is a measure of the corresponding change in the wire resistance. These two voltages form the input to the operational servo amplifier. The selected amplifier has an output current, $i$, which is inversely proportional to the resistance change of the hot-wire sensor. Feeding this current back to the top of the bridge restores the sensor's resistance to its original value. Modern amplifiers used in anemometers have a very fast response time $(\sim 450$ $\mathrm{kHz}$ ). Furthermore, in the $\mathrm{CT}$ mode, the sensor is maintained at a constant temperature except for very high frequency fluctuations. The CTA circuit operates on a bridge ratio of 1:20 and has a frequency response of $10 \mathrm{kHz}$ (3 dB limit). Various CTA and probe parameters used are listed in Table 4.1. A 55P16 single normal probe was used for all the single wire measurements. Various parameters given in Table 4.1 correspond to specifications for the SN probe (Dantec Dynamics), wherein the overheat ratio determines the working temperature $\left(T_{\text {wire }}\right)$ of the sensor. An overheat adjustment is based on the measured wire resistance at the ambient fluid temperature. The probe-CTA combination used during calibration was also not changed during the run to preserve the calibration. A variation as large as $25 \%$ in the bridge voltage was obtained if the probecircuit combination was altered.

\subsubsection{Calibration of a Single-Normal Hot-Wire Probe}

A calibration was performed to establish the relation between CTA output and flow velocity by exposing the probe to a set of known velocities, $U$, and then recording the voltages, $E$. A curve fit through the points $(E, U)$ gives the transfer function for 
converting data records from voltages into velocities. Due to individual features of each probe, variations in anemometer circuits and settings; and variations in the flow temperature, the hot-wire calibration may change. The calibration was thus checked at regular intervals and was re-performed if necessary. As a quick check before an experiment, a reference voltage reading was recorded by placing the probe in a known fluid velocity. The temperature of the flow as well as the ambient room temperature was logged. This was done to ensure that the reference point (voltage reading) during the test run matched with the calibration. Typically, calibration was either carried out in a dedicated probe calibrator or in a wind tunnel with a pitot-static tube as the velocity reference (Bruun 1996; Bruun \& Tropea 1985; Saddoughi \& Veeravalli 1996). The velocity was evaluated from a difference in the pressure reading, $\Delta P$, across the pitot static tube or a calibration nozzle. However at low speeds $(\leq 3 \mathrm{~m} / \mathrm{s})$, the value of $\Delta P$ in air becomes small $(<1 \mathrm{~mm}$ of water) and it was difficult to obtain accurate estimates of velocities by this method.

For the present study, a separate jet-flow calibration was used as shown in Figure 4.2. Similar methods has been used before in a number of investigations (Almquist \& Legath 1965; Andrews et al. 1972; Koppius \& Trines 1976; Lee \& Budwig 1991; Manca et al. 1988). A pressure regulator was used to control the supply from the compressed air line ( 100 psig). The air supply was fed to a proportioner meter (Model \# P21A1-BA2, Aalborg Corp.) which could control air flow rates upto $60 \mathrm{~L} / \mathrm{min}$. A 1/2" diameter PVC pipe was used to create a jet. The hot-wire was inserted towards the rear end so that it was well within the fully developed velocity profile. The temperature was 


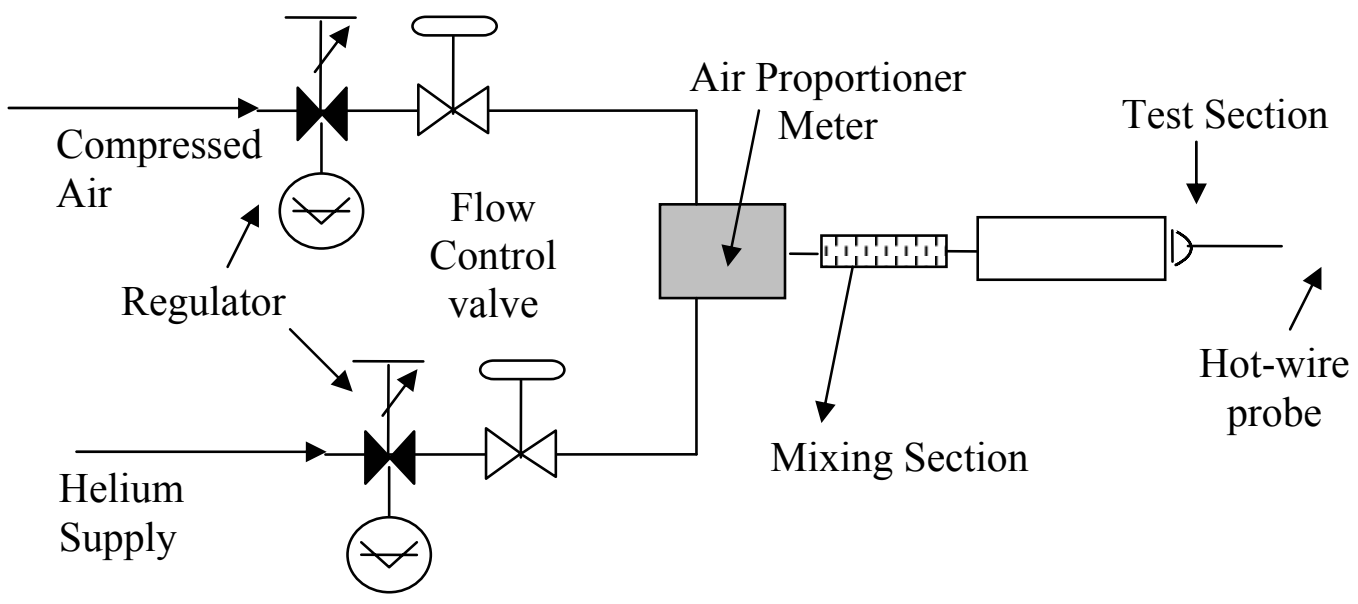

Figure 4.2 Schematic of setup used for hot-wire calibration. 
also recorded during calibration (Betti et al. 2001) by using a E-type thermocouple. Later, during the experiment, if the mean temperature of the flow ( $\left.T_{\text {fluid }}\right)$ during the experiment varied from the temperature during calibration, the CTA data records were corrected for temperature variations using Equation 4.1 (Kanevce \& Oka 1973).

$$
E_{\text {corr }}=\left(\frac{T_{\text {wire }}-T_{\text {fluid }}}{T_{\text {wire }}-T_{\text {calib }}}\right)^{0.5} \cdot E_{a}
$$

\subsubsection{Hot-Wire Calibration Equations}

In selecting the hot-wire response equation, we faced dual objectives of high accuracy and ease of use. Based on historical and physical considerations, we considered using a polynomial curve fit in the form $U=F(E)$ or a more traditional relationship from the original investigation by King (1914) which assumed a form $E=F(U)$.

(a) Polynomial curve fits: A 4th order polynomial plot was fitted to this data and found to have a $R^{2}$. value of 0.9999 . Errors associated with the 4 th order fit in Figure $4 \mathrm{~b}$ can be attributed to errors in velocity measurement inside the gas channel. For comparison purposes, the goodness of fit of the selected hot-wire response equation was described by the related normalized standard deviation, $\varepsilon_{\mathrm{u}, \text {, which was defined as: }}$

$$
\varepsilon_{u}=\left[\frac{1}{N} \sum_{i=1}^{N}\left(1-\frac{U}{U_{\text {calib }}}\right)^{2}\right]^{\frac{1}{2}}
$$

The value of $\varepsilon_{\mathrm{u}}$ was tabulated in Table 4.2 for polynomial fits of order 2 - 4 .

(b) King's Law Fits: The relationship between voltage and velocity was often assumed to be of the form: 


$$
E^{2}=A+B U^{n}
$$

King's analysis suggested using an exponent of $n=0.5$. However, Collis and Williams (1959) have shown a better curve fit using a value of $n=0.45$ for $0.02<R e<44$. For a small micron sized wire, the velocity range used by Collis and Williams was large. So it was decided to evaluate $n_{\mathrm{opt}}$ for the velocity range of our interest (0-3 m/s). A straight line fit by varying the exponent $\mathrm{n}$ was performed and the parameter $\varepsilon_{\mathrm{u}}$ was evaluated over the range. $n_{\mathrm{opt}}$ was chosen based on the exponent which returned the lowest value of the normalized standard deviation. The results were plotted in Figure 4.3. It should be noted here that since we use a smaller velocity range for our calibration, we obtained a value of $n_{\mathrm{opt}}=0.5$ which was different from the value obtained by Collis and Williams (1959).

Our use of a hot-wire in a Rayleigh-Taylor flow demanded the calibration of the wire in a binary mix of air and helium. An extensive literature survey was also conducted to study the response of a hot-wire in a flow consisting of a variable concentration of binary gas mixture (Aihara et al. 1967; Chassaing et al. 1994; Corrisin 1949; Harion et al. 1996; Pitts \& McCaffrey 1986; Stanford \& Libby 1974). However, there was a lack of heat transfer correlations in the literature for a binary air-helium gas mixture. So we decided to formulate basic heat transfer correlations to fit our air-helium calibration data. The basis of these measurements was a modification of the Collis and Williams correlation (1959) that accounts for heat transfer on hot-wire performance. The modification accounts for the effects of velocity and density simultaneously in a binary (Helium/Air) gas mixture. The modified Collis Williams equation, illustrates that 


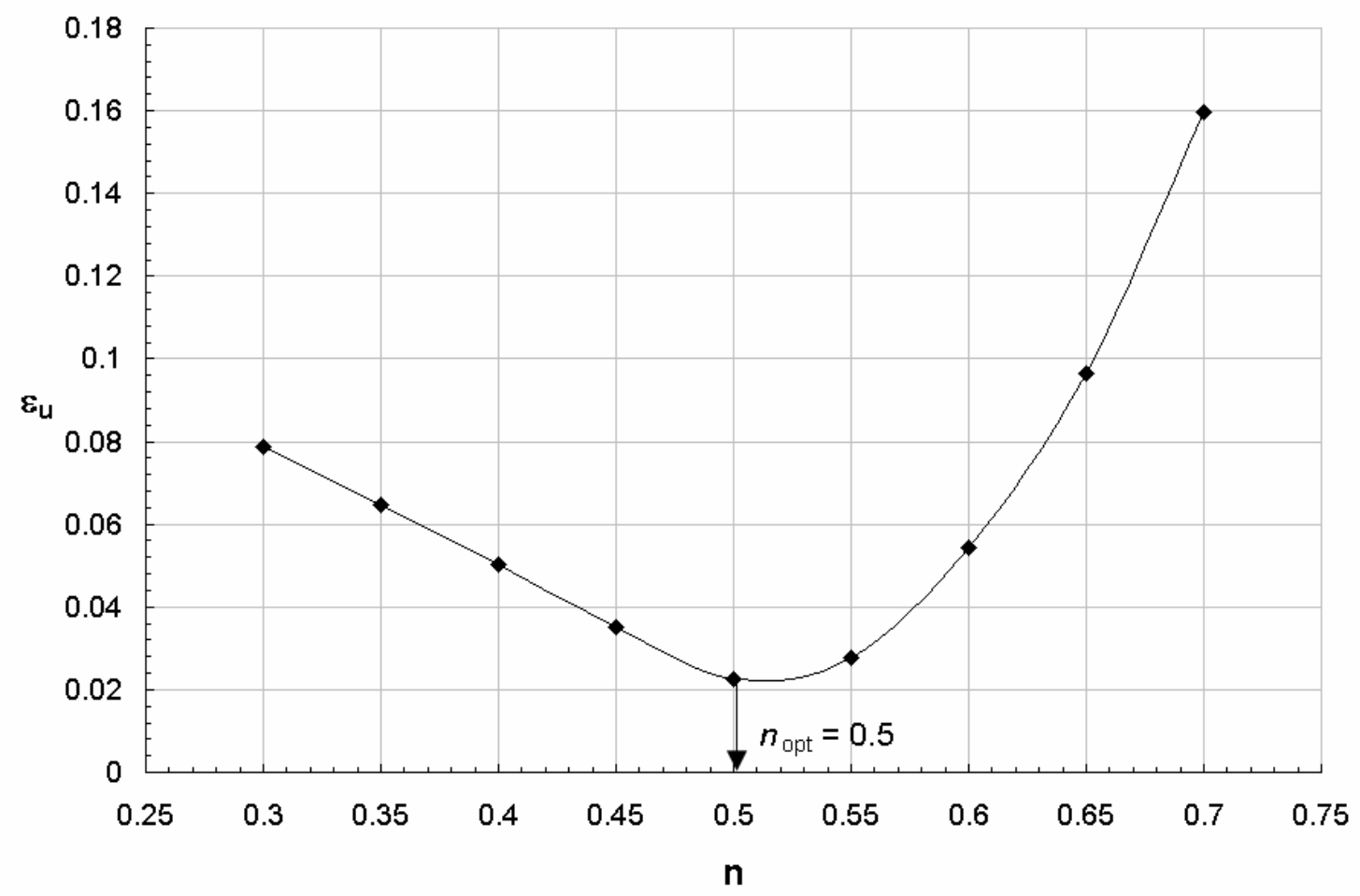

Figure 4.3 Variation in $\varepsilon_{\mathrm{u}}$ (goodness of fit) with exponent in power law relationship from $\mathrm{SN}$ wire calibration data in air at overheat ratio 0.6 over a velocity range of $0.2-3 \mathrm{~m} / \mathrm{s}$. 
TABLE 4.2 Curve fits in the velocity range $0-3 \mathrm{~m} / \mathrm{s}$ (calculated at overheat ratio 1.6)

\begin{tabular}{|l|c|c||}
\hline \multicolumn{1}{|c|}{ Equation } & $\varepsilon_{\mathbf{u}}$ & $\boldsymbol{R}^{2}$ \\
\hline King's Law Fit: $E=A+B U^{n} \cdot\left(n=n_{\mathrm{opt}}=0.5\right)$ & 0.02259 & 0.9992 \\
\hline Polynomial Fit : $U=A+B E+C E^{2}$ & 0.05466 & 0.9998 \\
\hline Polynomial Fit : $U=A+B E+C E^{2}+D E^{3}$ & 0.01337 & 0.9999 \\
\hline Polynomial Fit : $U=A+B E+C E^{2}+D E^{3}+F E^{4}$ & 0.00868 & 0.9999 \\
\hline
\end{tabular}




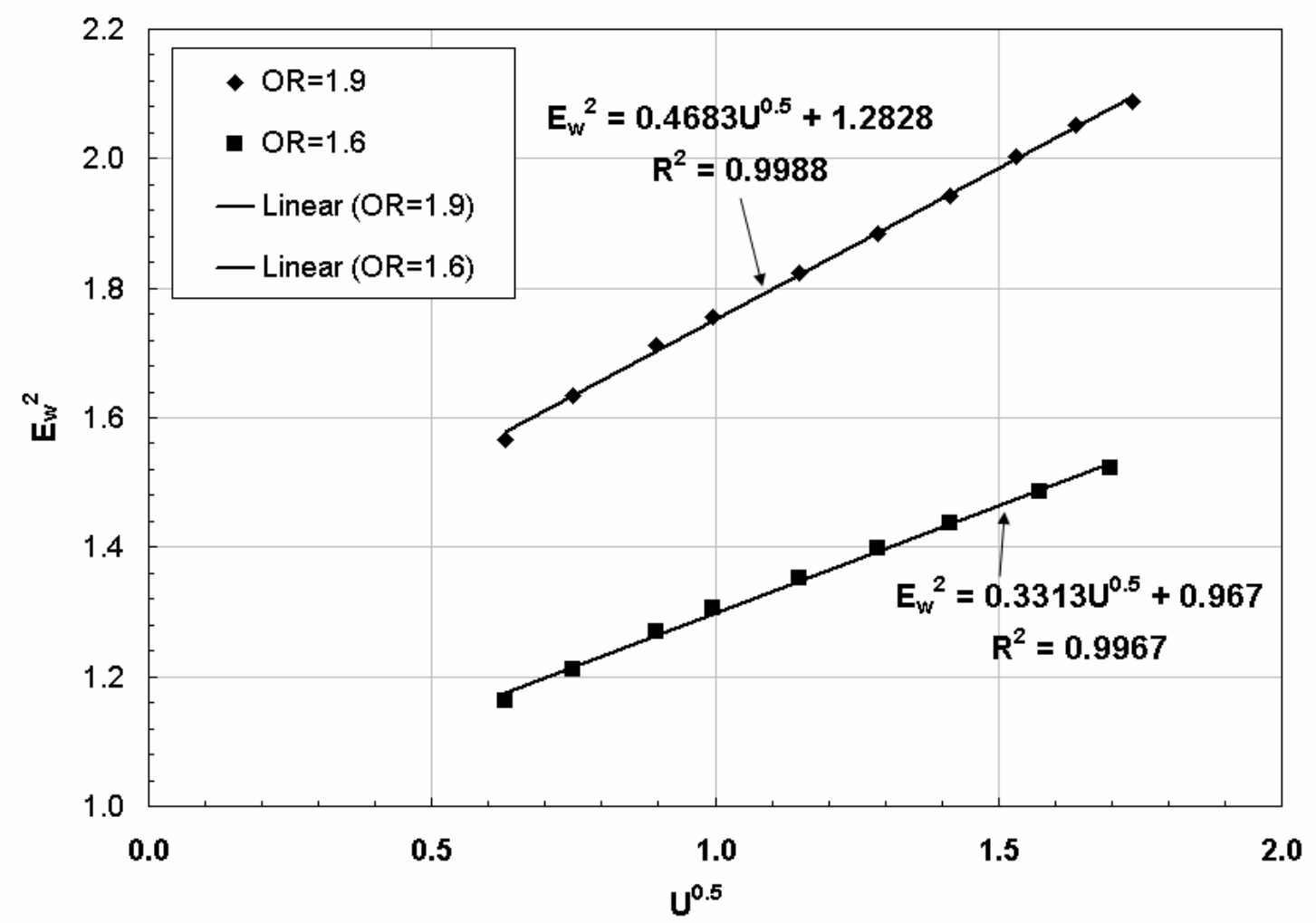

Figure 4.4 Hot-wire calibration data (King's law fit) in air at overheat ratio 1.9 $\left(\Delta T=257.14^{\circ} \mathrm{C}\right)$ and $1.6\left(\Delta T=171.43^{\circ} \mathrm{C}\right)$. 


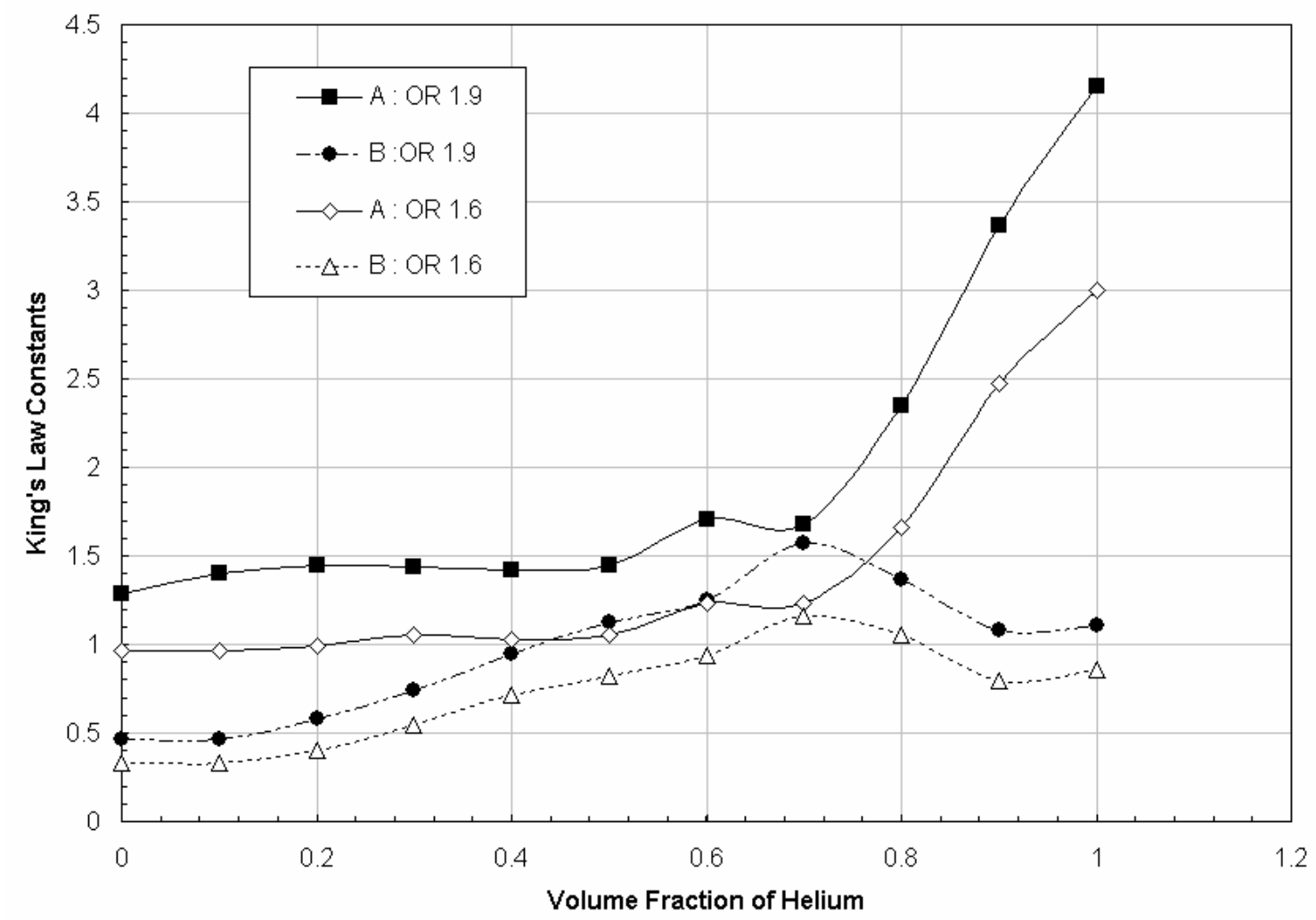

Figure 4.5 Variation in King's Law constants for different volume fractions of helium in a binary air-helium mixture. The plots are for overheat Ratio $1.9\left(\Delta T=257.14^{\circ} \mathrm{C}\right)$ and 1.6 $\left(\Delta T=171.43^{\circ} \mathrm{C}\right.$ ). (Note: The constants $A, B$ were obtained from a linear fit of $E^{2}$.vs. $U^{0.5}$. for a volume fraction range of $\phi=0 \rightarrow 1 . R^{2}$. values for these fits range from 0.9930 to 0.9993. A representative calibration plot for $E^{2}$.vs. $U^{0.5}$ for air is provided in Figure 4.4) 
the rate of heat transfer from the wire is dependent on the velocity as well as the properties of the cooling fluid, and is written as:

$$
N u=\left(T_{\text {film }} / T_{\text {fluid }}\right)^{0.17}\left[0.24\left(\operatorname{Pr}_{\text {mix }} / \operatorname{Pr}_{\text {air }}\right)^{0.2}+0.56\left(\operatorname{Pr}_{\text {mix }} / \operatorname{Pr}_{\text {air }}\right)^{0.33} \operatorname{Re}_{d}^{0.45}\right]
$$

where, $N u$ is the Nusselt number, $T_{\text {film }}$ is the average of the wire temperature $\left(T_{\text {wire }}\right)$ and the fluid (mix) temperature $\left(T_{\text {fluid }}\right), R e_{\mathrm{d}}$ is the Reynolds number and $\operatorname{Pr}$ the Prandtl number. All the properties were evaluated at $T_{\text {film. }}$. Thus a linear (King's Law) fit was applied to all calibration data as it was easy to use for formulating correlations of the type described in Equation 4.4. Figure 4.4 shows a calibration plot in air at overheat ratio 1.9 and 1.6. It was seen that the response was fairly linear over the range of velocities under consideration. Figure 4.5 plots the slope and intercept obtained from a King's Law fit as a function of the volume fraction of helium in a binary air-helium mixture. Details about the heat transfer correlations can be found in section 5 .

\subsection{Constant Current Anemometry (CCA) for Measuring Temperature Fluctuations}

\subsubsection{The Need for a CCA Unit}

In the present experiment, the density gradient needed to create a R-T Instability was obtained by using a heavy fluid (air) over a light one (air-helium mixture). For the hotwire measurements, it was important to locate the position of the probe in the mix (Banerjee \& Andrews 2006; Mueschke 2004; Ramaprabhu \& Andrews 2004; Wilson \& Andrews 2002). The addition of helium to the mix caused a small temperature gradient $\left(\sim 2^{\circ} \mathrm{C}\right)$ along the vertical $(y)$ direction (i.e. from the air to the air/helium mixture). An electric coil heater $(1500 \mathrm{~W})$ was used to heat the air stream, thus creating an overall 
gradient of $\sim 4^{\circ} \mathrm{C}$. We took advantage of this small temperature gradient and initially used thermocouples to check for the centerline of the mix. The thermocouple temperature diagnostics consisted of an E-type thermocouple (40 gauge wire of nickelchromium and constantan) that was positioned at different downstream locations in the channel to ensure that the center line of the mix remains horizontal (Mueschke 2004; Ramaprabhu \& Andrews 2004; Wilson \& Andrews 2002). However, the time response of the E-type thermocouple was found $\sim 20 \mathrm{~Hz}$. So this was discontinued and a $5 \mu \mathrm{m} \mathrm{SN}$ wire probe (Model \#55P16) was used as a cold wire probe coupled to a Constant Current Anemometer unit (A.A. Laboratories, Inc.). At the start of the experiment, the heater was switched ON and the cold wire was placed close to the geometric centerline of the mixing layer by visual inspection and the data was logged. The centerline offset factor $\phi$ (as given in Equation 4.5) was computed after the logging process as:

$$
\phi=\left[1+\frac{C_{p}^{\text {bottom }}}{C_{p}^{\text {top }}} \frac{\rho_{\text {bottom }}}{\rho_{\text {top }}}\left(\frac{T_{\text {bottom }}-T_{i}}{T_{i}-T_{\text {top }}}\right)\right]^{-1}
$$

where, $C_{p}^{\text {bottom }}$ and $T_{\text {bottom }}$ were the specific heat and temperature of the helium-air mixture (flow at the bottom section of the channel); $C_{p}^{\text {top }}$ and $T_{\text {top }}$ were the specific heat and temperature of air (flow at the top section of the channel); and $T_{i}$ was the temperature of the mix. If $\phi \neq 0.5$, the position of the cold-wire was readjusted and the data logging process repeated. The cold-wire position was adjusted until $\phi$ approaches a value of 0.5 with an accuracy of 5\%. Once the centerline was found, the heater was switched OFF. The hot-wire measurements were then taken at the determined location. 


\subsubsection{The Cold (Resistance) Wire Probe}

Over a limited temperature range, the resistance of a wire can be approximated as a linear function of temperature, i.e.,

$$
R_{\text {wire }}=R_{20}\left[1+\alpha_{20}\left(T_{\text {wire }}-20\right)\right]
$$

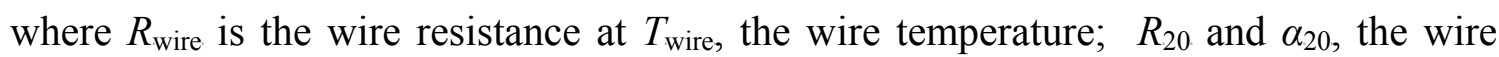
resistance and the temperature coefficient of resistivity at a reference temperature of $20^{\circ} \mathrm{C}$. To maintain a linear relationship as depicted by Equation 4.6a, the reference temperature should be chosen in the range of interest. The resistance $R_{20}$ is related to the sensor dimensions, $l_{\text {wire }}$ (length of the wire) and $d_{\text {wire }}$ (wire diameter) based on the relation:

$$
R_{20}=\frac{\sigma_{20} l_{\text {wire }}}{\pi d_{\text {wire }}^{2} / 4}
$$

The resistance fluctuations sensed by the wire as the fluid flows over it was converted to voltage fluctuations by passing a small current, $I$, through the sensor. The produced voltage fluctuations, $e(t)$, are related to the temperature fluctuations in the fluid by the relation:

$$
e(t)=\alpha_{20} I R_{20}\left[T_{\text {wire }}(t)-T_{20}\right]
$$

Measurement of low amplitude, small scale and high frequency temperature fluctuations are important in turbulence research. Such measurements have been made possible by use of low noise, high frequency AC Wheatstone bridges (LaRue et al. 1975). The temperature calibration of a resistance-wire was used to determine the calibration constants in the relationship as shown in Figure 4.6. 


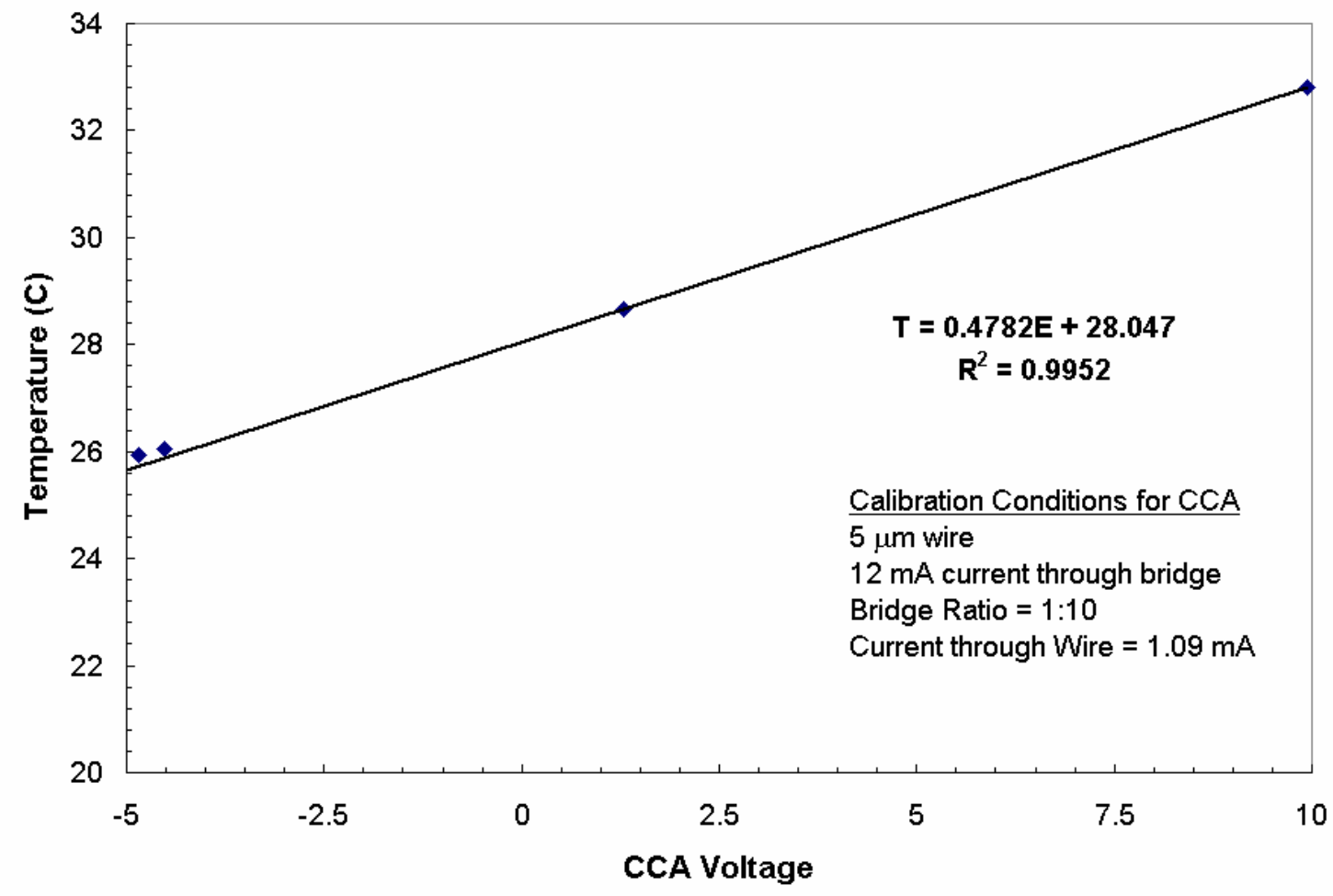

Figure 4.6 Calibration curve for cold wire (resistance wire) probe. 


\subsection{Single Wire Measurements}

For the Rayleigh-Taylor mix experiments under consideration, velocity fluctuations in all three mutually perpendicular directions are significant. When using a hot-wire probe with a CTA unit, there are a number of methods by which the velocity fluctuations can be resolved and measured accurately. One method uses a 3-wire probe to resolve all 3 components. However, besides the probe being expensive, the 3-wire method requires an accurate calibration (velocity, density and directional) of the probe. An alternate method resolves the three velocity components by using a multi-position single wire technique (Bruun 1972). This method does not require complicated calibration and is easy to implement. However, Bruun's method does not incorporate density effects. Measurements using this method is thus limited only to the low Atwood measurements $\left(A_{t} \sim 0.035\right)$ which contains less than $3.3 \%$ by volume. This was used as a first trial. Errors associated with this assumption are described in Appendix B.7.1. A multiposition multi-overheat (mpmo) single wire technique, which incorporates the effect of a binary gas mixture of air and helium is used (Corrisin 1949; Kovasznay 1950). This technique requires extensive calibration to take into account effects of density variation in our flow. Both the techniques are described in details in the following sections.

\subsubsection{Multi-position Single Wire Technique}

H.H. Bruun (1972) used a multi-position single-wire technique by holding a SN wire at different orientations to the flow to measure all the 3 velocity components. The components were not measured simultaneously but by orienting the hot-wire (directions given in Table 4.3) and ensuring that the probe was at the same spatial location. In his 
analysis, Bruun used a flow that was two-dimensional $(W=0)$ with three dimensional fluctuations. However, since the mean flow field used in the present experiment was one-dimensional ( $V=W=0)$, the technique of Bruun reduced to three measurements instead of the six roll positions required to resolve the two dimensional mean flow. Following Bruun, the velocity vector $\vec{V}$ was taken to have the velocity components $\left(U+u^{\prime}, v^{\prime}, w^{\prime}\right)$. Taking into consideration a contribution from the angle of incidence, the equations were resolved to determine the 3 different orientations as given in Table 4.3. For a wire normal to the mean flow direction, with wire support parallel to the flow (position 1), the mean component $U_{\mathrm{m}}$, is given by:

$$
U_{m}=U\left[1+\frac{1}{2} \frac{W^{2}}{U^{2}}+\frac{1+2 b}{2} \frac{\overline{w^{\prime 2}}}{U^{2}}-\frac{W}{U} \frac{\overline{u^{\prime} w^{\prime}}}{U^{2}}-\frac{1+2 b}{2} \frac{\overline{u^{\prime} w^{\prime 2}}}{U^{3}}\right]
$$

Since $W \approx 0$ and ignoring 3rd order terms, Equation 4.8 simplifies to the form for the mean component of the flow as:

$$
U_{m}=U\left[1+\frac{1+2 b}{2} \frac{\overline{w^{\prime 2}}}{U^{2}}\right]
$$

In Equations 4.8 and 4.9, ' $b$ ' is a constant of calibration for the probe and is calculated from the following relation:

$$
U_{ \pm \gamma}=U\left(1+b \cdot \sin ^{2} \gamma\right)
$$

where $U_{ \pm \gamma}, U$ are the velocities measured during calibration in air. The fluctuating component measured by a hot-wire placed in position 1 (see Table 4.3 and Figure 4.7) can be written as: 


$$
\overline{u_{m}^{\prime 2}}=\overline{u^{\prime 2}}\left[1+2 \frac{W}{U} \frac{\overline{u^{\prime} w^{\prime}}}{\overline{u^{\prime 2}}}+(1+2 b) \frac{\overline{u^{\prime} w^{\prime 2}}}{\overline{u^{\prime 2} U}}-(1+2 b) \frac{\overline{u^{\prime 2} w^{\prime 2}}}{\overline{u^{\prime 2}} U^{2}}\right]
$$

Again, since $W \approx 0$ and ignoring higher order terms, Equation 4.11 simplifies to:

$$
\overline{u_{m}^{\prime 2}}=\overline{u^{\prime 2}}
$$

For a wire yawed to mean flow direction at an angle $\pm \gamma$ (position 2 and 3, see Table 4.3 and Figure 4.5), the mean component can be expressed as:

$$
U_{ \pm \gamma}=U\left(\cos ^{2} \gamma+k^{2} \sin ^{2} \gamma\right)^{\frac{1}{2}}\left[1+\frac{1+2 b+A \tan ^{2} \gamma}{2} \frac{\overline{w^{\prime 2}}}{U^{2}}-\frac{1+2 b+A \tan ^{2} \gamma}{2} \frac{\overline{u^{\prime} w^{\prime 2}}}{U^{3}}\right]
$$

where $b$ is the constant of calibration defined earlier, $k$ the yaw coefficient and, $A$, a coefficient which accounts for the effect of yaw dependence on measurements.

$$
\begin{gathered}
k=\frac{1}{\sin \gamma}\left[\left(\frac{E_{\gamma= \pm 45^{\circ}}^{2}-E_{U=0}^{2}}{E_{\gamma=0^{\circ}}^{2}-E_{U=0}^{2}}\right)^{\frac{2}{0.5}}-\cos ^{2} \gamma\right]^{\frac{1}{2}} \\
A=\frac{\cos ^{2} \alpha \cdot\left(1-k^{2}\right)}{\cos ^{2} \alpha \cdot\left(1-k^{2}\right)+k^{2}}
\end{gathered}
$$

Ignoring 3rd order terms, and simplifying, gives:

$$
U_{ \pm \gamma}=U\left(\cos ^{2} \gamma+k^{2} \sin ^{2} \gamma\right)^{\frac{1}{2}}\left[1+\frac{1+2 b+A \tan ^{2} \gamma}{2} \frac{\overline{w^{\prime 2}}}{U^{2}}\right]
$$



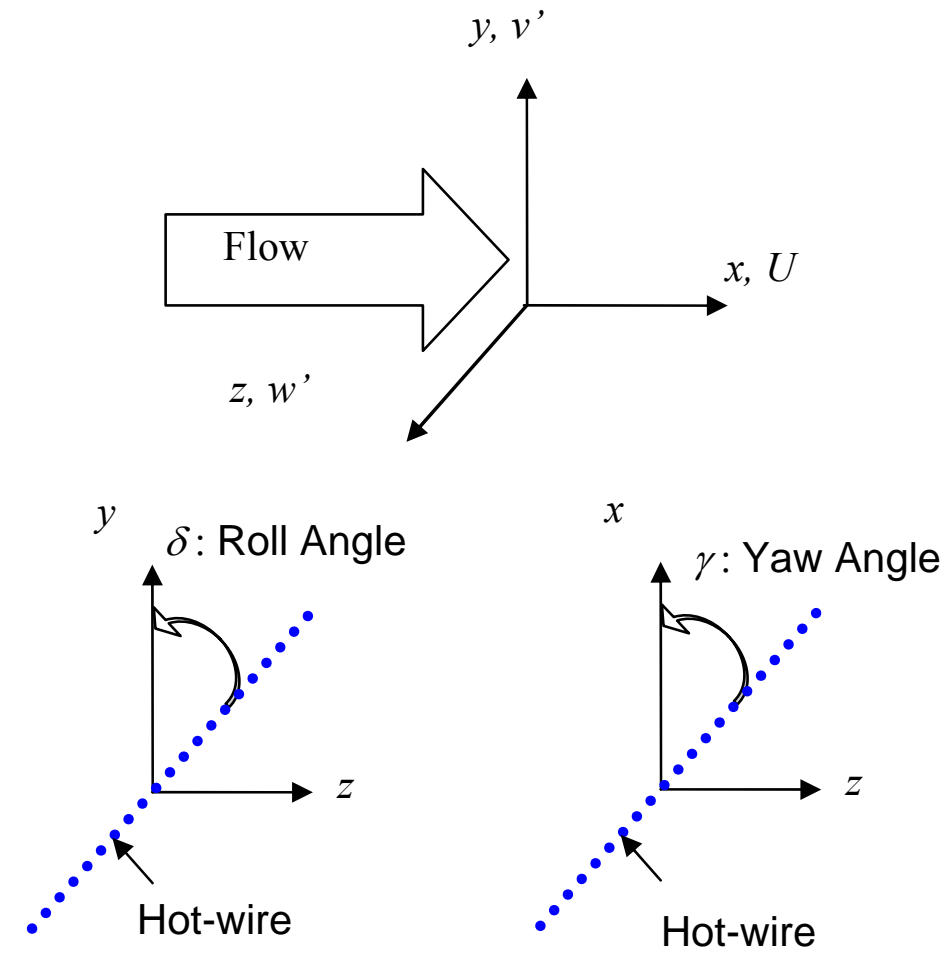

Figure 4.7 Co-ordinate system for measurements and various orientations of hot-wire used for measurements (probe axis was normal to the dotted line). 
TABLE 4.3 Various measurement orientations for multi-position measurement technique. The measurement uncertainty of the positions is $\pm 2.5^{\circ}$

\begin{tabular}{|c|c|c|}
\hline Position & $\boldsymbol{\gamma}$ & $\boldsymbol{\delta}$ \\
\hline 1 & $0^{\circ}$ & $0^{\circ}$ \\
\hline 2 & $45^{\circ}$ & $90^{\circ}$ \\
\hline 3 & $-45^{\circ}$ & $90^{\circ}$ \\
\hline
\end{tabular}


In addition, the fluctuating component (ignoring 3rd and 4th order terms) can be written as:

$$
\overline{u_{ \pm \gamma}^{\prime 2}}=\left(\cos ^{2} \gamma+k^{2} \sin ^{2} \gamma\right)\left(\overline{u^{\prime 2}}+A^{2} \tan ^{2} \gamma \cdot \overline{v^{\prime 2}} \mp 2 A \tan \gamma \cdot \overline{u^{\prime} v^{\prime}}\right)
$$

A value for $\overline{v^{2}}$ was obtained by adding Equations 4.16 for $\gamma=+45^{\circ}$ and $-45^{\circ}$ to get:

$$
\overline{v^{\prime 2}}=\frac{\overline{u_{+45}^{\prime 2}}+\overline{u_{-45}^{\prime 2}}-\left(1+k^{2}\right) \overline{u^{\prime 2}}}{\left(1+k^{2}\right) A^{2}}
$$

Thus, using Equations 4.12, 4.15 and 4.17, the values of $\overline{u^{\prime 2}}, \overline{v^{\prime 2}}$ and $\overline{w^{\prime 2}}$ can be evaluated. The values of $k$ and $A$ were evaluated by performing a yaw-calibration for the wire (Bruun 1972; Bruun \& Tropea 1985).

\subsubsection{Multi-position Multi-Overheat Single Wire Technique}

The mean voltage across a wire oriented at an angle to the flow can be written as:

$$
E=f(u, v, w, \rho)
$$

The total change in the mean voltage, $\mathrm{E}$, due to change in the independent variables is:

$$
\begin{aligned}
d E & =\left(\frac{\partial E}{\partial u}\right)_{v, w, \rho} d u+\left(\frac{\partial E}{\partial v}\right)_{u, w, \rho} d v+\left(\frac{\partial E}{\partial w}\right)_{u, v, \rho} d w+\left(\frac{\partial E}{\partial \rho}\right)_{u, v, w} d \rho \\
& =\Delta e_{U} d u+\Delta e_{v} d v+\Delta e_{w} d w+\Delta e_{\rho} d \rho
\end{aligned}
$$

For small fluctuations in velocity and density, it was assumed that the wire fluctuation can be written as (Rose 1973):

$$
\frac{e^{\prime}}{E}=\Delta e_{U} \frac{u^{\prime}}{\bar{U}}+\Delta e_{V} \frac{v^{\prime}}{\bar{U}}+\Delta e_{w} \frac{w^{\prime}}{\bar{U}}+\Delta e_{\rho} \frac{\rho^{\prime}}{\bar{\rho}}
$$


The wire sensitivities are evaluated based on calibration of the wires and are defined as follows:

$$
\begin{aligned}
& \Delta e_{U}=\left.\frac{\partial \ln E}{\partial \ln U}\right|_{\theta, \Delta T_{w}, \rho}=\left.\frac{U}{E} \frac{d E}{d U}\right|_{\theta, \Delta T_{w}, \rho} \\
& \Delta e_{v}=\left.\frac{\partial \ln E}{\partial \theta}\right|_{u, \Delta T_{w}, \rho}=\left.\frac{1}{E} \frac{d E}{d \theta}\right|_{u, \Delta T_{w}, \rho} \\
& \Delta e_{\rho}=\left.\frac{\partial \ln E}{\partial \rho}\right|_{u, \Delta T_{w}, \theta=0}=\left.\frac{\rho}{E} \frac{d E}{d \rho}\right|_{u, \Delta T_{w}, \theta=0}
\end{aligned}
$$

Squaring and taking time-average of Equation 4.20a, we get:

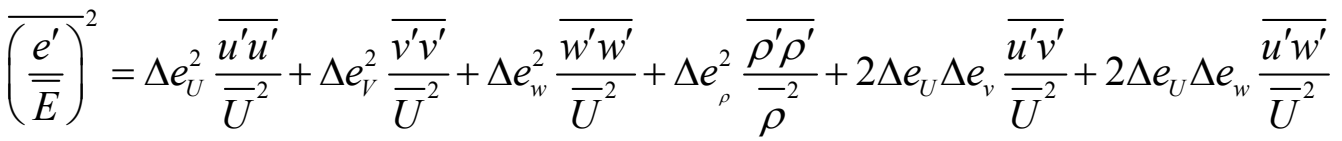

$$
\begin{aligned}
& +2 \Delta e_{w} \Delta e_{v} \frac{\overline{w^{\prime} v^{\prime}}}{\bar{U}^{2}}+2 \Delta e_{U} \Delta e_{\rho} \frac{\overline{\rho^{\prime} u^{\prime}}}{\bar{\rho} \bar{U}}+2 \Delta e_{V} \Delta e_{\rho} \frac{\overline{\rho^{\prime} v^{\prime}}}{\bar{\rho} \bar{U}}+2 \Delta e_{w} \Delta e_{\rho} \frac{\overline{\rho^{\prime} w^{\prime}}}{\bar{\rho} \bar{U}}
\end{aligned}
$$

Equation 4.21 has 10 independent terms on the right hand side. Thus 10 independent measurements are needed to evaluate all these variables. By using the current technique, $\overline{u^{\prime 2}}$ and $\overline{v^{\prime 2}}$ are measured and $\overline{u^{\prime 2}}=\overline{w^{\prime 2}}$ is taken from symmetry of the coherent structures. We also assume $\overline{\rho^{\prime} u^{\prime}}=\overline{\rho^{\prime} w^{\prime}}$ from symmetry. The cross terms in the Reynolds stress tensor is assumed to be of the same order, i.e. $\overline{v^{\prime} w^{\prime}} \sim \overline{u^{\prime} w^{\prime}} \sim \overline{u^{\prime} v^{\prime}}$. Thus Equation 4.21 can be simplified to:

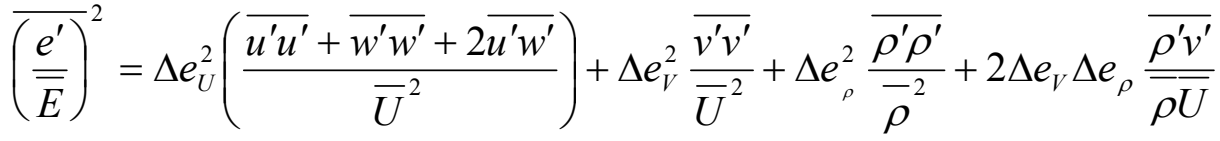

$$
\begin{aligned}
& +2 \Delta e_{U} \Delta e_{\rho}\left(\frac{\overline{\rho^{\prime} u^{\prime}}+\overline{\rho^{\prime} w^{\prime}}}{\bar{\rho} \bar{U}}\right)+2 \Delta e_{U} \Delta e_{v}\left(\frac{\overline{u^{\prime} v^{\prime}}+\overline{w^{\prime} v^{\prime}}}{\bar{U}^{2}}\right)
\end{aligned}
$$


Upon further simplification:

$$
\begin{aligned}
\overline{\left(\frac{e^{\prime}}{\bar{E}}\right)^{2}}= & \left(\frac{4 \Delta e_{U}^{2}}{\bar{U}^{2}}\right) \overline{u^{\prime} u^{\prime}}+\left(\frac{\Delta e_{V}^{2}}{\bar{U}^{2}}\right) \overline{v^{\prime} v^{\prime}}+\left(\frac{\Delta e_{\rho}^{2}}{\bar{\rho}^{2}}\right) \overline{\rho^{\prime} \rho^{\prime}}+\left(\frac{2 \Delta e_{V} \Delta e_{\rho}}{\bar{\rho} \bar{U}}\right) \overline{\rho^{\prime} v^{\prime}} \\
& +\left(\frac{2 \Delta e_{U} \Delta e_{\rho}}{\bar{\rho} \bar{U}}\right) \overline{\rho^{\prime} u^{\prime}}+\left(\frac{2 \Delta e_{U} \Delta e_{v}}{\bar{U}^{2}}\right) \overline{u^{\prime} v^{\prime}}
\end{aligned}
$$

Equation 4.23 has 6 independent terms. 6 independent measurements are needed to evaluate all these variables. Thus:

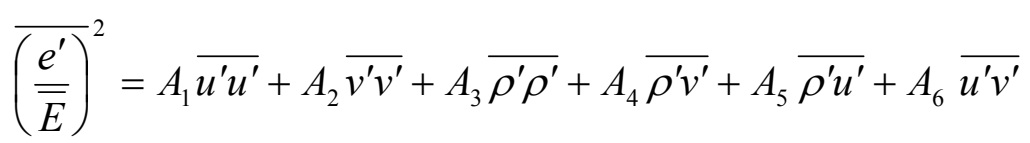

where, the constants $A_{\mathrm{i}}$ 's $(i=1-6)$ were defined as follows:

$$
\begin{gathered}
A_{1}=\frac{4 \Delta e_{U}^{2}}{\bar{U}^{2}}=\frac{1}{U^{2}}\left(\frac{U}{E} \frac{d E}{d U}\right)^{2}=\left(\frac{1}{E} \frac{d E}{d U}\right)^{2} \\
A_{2}=\frac{\Delta e_{V}^{2}}{\bar{U}^{2}}=\frac{1}{U^{2}}\left(\frac{1}{E} \frac{d E}{d \theta}\right)^{2}=\left(\frac{1}{E U} \frac{d E}{d \theta}\right)^{2} \\
A_{4}=\frac{2 \Delta e_{v} \Delta e_{\rho}}{\bar{\rho} \bar{U}}=\frac{2}{\bar{\rho} \bar{U}}\left(\frac{\rho}{E} \frac{d E}{d \rho}\right)\left(\frac{1}{E} \frac{d E}{d \theta}\right)=\frac{1}{\bar{\rho}^{2}}\left(\frac{\rho}{E} \frac{d E}{d \rho}\right)^{2}=\left(\frac{1}{E} \frac{d E}{d \rho}\right)^{2} \\
A_{5}=\frac{4 \Delta e_{U} \Delta e_{\rho}}{\bar{\rho} \bar{U}^{2}}=\frac{4}{\bar{\rho} \bar{U}}\left(\frac{U}{E} \frac{d E}{d U}\right)\left(\frac{\rho}{E} \frac{d E}{d \rho}\right)=\frac{4}{E^{2}}\left(\frac{d E}{d U}\right)\left(\frac{d E}{d \rho}\right) \\
A_{6}=\frac{4 \Delta e_{U} \Delta e_{v}}{\bar{U}^{2}}=\frac{4}{\bar{U}^{2}}\left(\frac{U}{E} \frac{d E}{d U}\right)\left(\frac{1}{E} \frac{d E}{d \theta}\right)=\frac{4}{E^{2} U}\left(\frac{d E}{d U}\right)\left(\frac{d E}{d \theta}\right)
\end{gathered}
$$


In a R-T mix, the cross correlation term $\overline{u^{\prime} v^{\prime}}$ is negligible since the mushroom-shaped structures have left-right symmetry about the center, so that $\left.u^{\prime} v^{\prime}\right|_{\text {right }}=-\left.u^{\prime} v^{\prime}\right|_{\text {left }}$ (Ramaprabhu \& Andrews 2004). Similarly, for $u$ - and $w$-components, $\overline{v^{\prime} w^{\prime}}$ and $\overline{u^{\prime} w^{\prime}} \sim 0$. Thus, $\quad \overline{u_{i} u_{j}}=\left(\begin{array}{ccc}\overline{u^{\prime 2}} & 0 & 0 \\ 0 & \overline{v^{\prime 2}} & 0 \\ 0 & 0 & \overline{w^{\prime 2}}\end{array}\right)$

Using the assumptions for the RT flow reduces the total number of independent measurements needed to 5. Thus, by using a multi (3)-position (see Figure 4.8) and multi (2)-Overheat method, we can get 6 independent traces from which we can calculate the quantities: $\overline{u^{\prime 2}}, \overline{v^{\prime 2}}, \overline{\rho^{\prime 2}}, \overline{\rho^{\prime} u^{\prime}}$ and $\overline{\rho^{\prime} v^{\prime}}$. It should be noted that the choice of overheat ratio of the wire and the orientations must be independent enough to be able to measure the correlations effectively. The details of the calibration techniques are discussed in details in the following section. Since the method required measurements at multiple overheats and multiple orientations, an extensive velocity, density and directional calibration was undertaken at each overheats. Since the technique involves solving linear equations, the coefficients ( $A_{\mathrm{i}}$ 's) for each measurement should be sufficiently independent. The choice of overheats (1.9 and 1.6) was dependent on this criteria. The wire properties at these overheat and the jumper settings for the CTA Bridge are given in Table 4.4. To evaluate the sensitivities $d E / d U$ and $d E / d \rho$, the density of the fluid is varied by controlling the proportion of the two gases (air and helium) by using the air 
TABLE 4.4 Wire properties at overheat ratios 1.9 and 1.6

\begin{tabular}{|c|c|c|}
\hline \multicolumn{3}{|l|}{ Probe specific parameters } \\
\hline Sensor resistance, $R_{20}(\Omega)$ & \multicolumn{2}{|c|}{3.38} \\
\hline Sensor lead resist., $R_{\mathrm{L}}(\Omega)$ & \multicolumn{2}{|c|}{0.9} \\
\hline Support resistance, $R_{\mathrm{S}}(\Omega)$ & \multicolumn{2}{|c|}{0.44} \\
\hline Cable resistance, $R_{\mathrm{c}}(\Omega)$ & \multicolumn{2}{|c|}{0.2} \\
\hline Sensor TCR, $\alpha_{20}(/ \mathrm{K})$ & \multicolumn{2}{|c|}{0.0036} \\
\hline \multicolumn{3}{|l|}{ Wire operating parameters } \\
\hline Overheat Ratio & 1.9 & 1.6 \\
\hline$\Delta T=T_{\mathrm{w}}-T_{a m b}\left({ }^{\circ} \mathrm{C}\right)$ & 257.14 & 171.43 \\
\hline Operating resistance, $R_{\mathrm{w}}(\Omega)$ & 6.51 & 5.47 \\
\hline Total resistance, $R_{\mathrm{T}}(\Omega)$ & 7.99 & 6.98 \\
\hline Decade resistance, $R_{\mathrm{D}}(\Omega)$ & 159.83 & 139.55 \\
\hline Jumper settings & dduu-uuud & ddud-uuud \\
\hline Bridge ratio, $M$ & \multicolumn{2}{|c|}{$1: 20$} \\
\hline
\end{tabular}



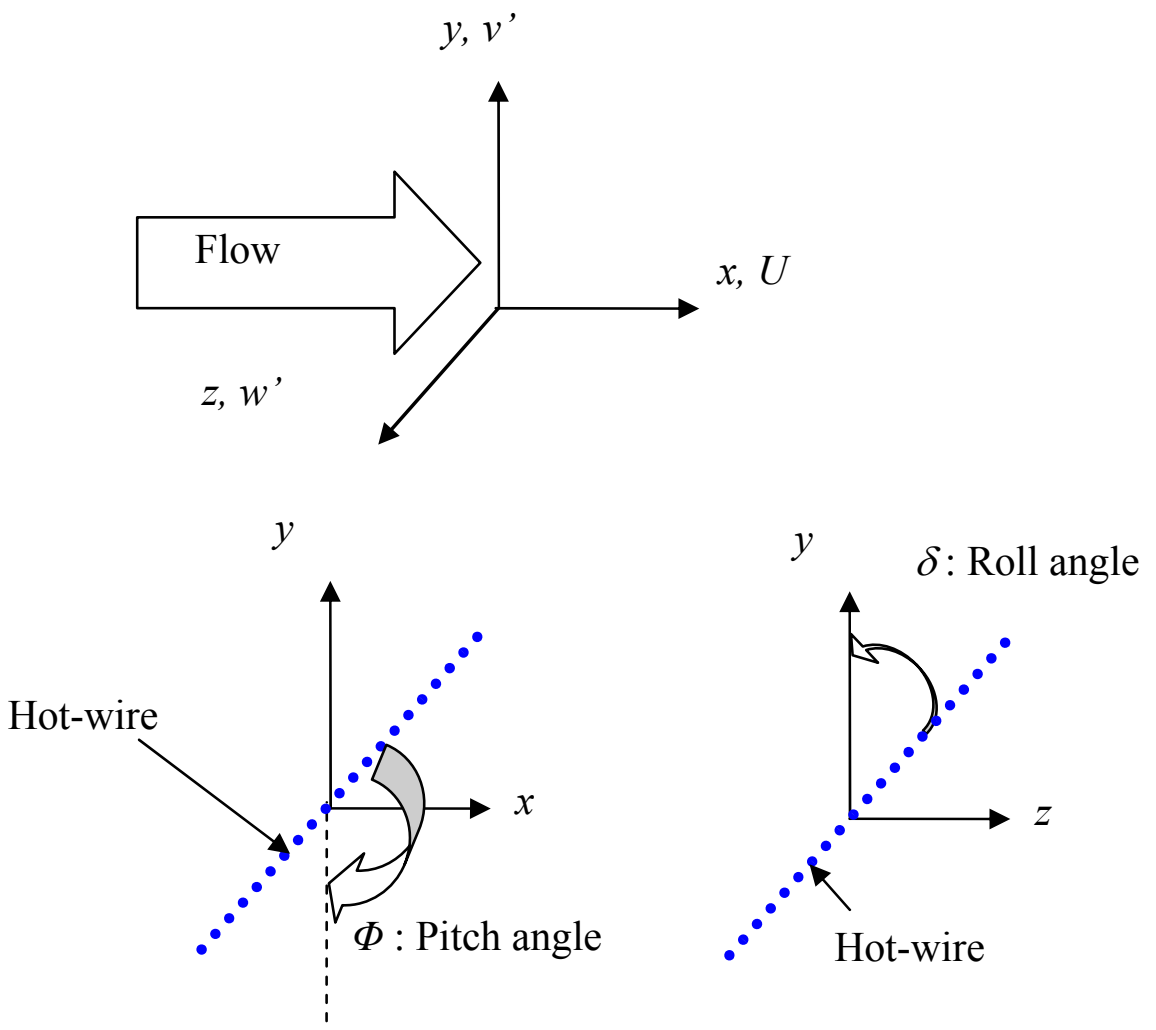

Figure 4.8 Wire orientations for multi-overheat multi-position technique. 
TABLE 4.5 Various measurement orientations for multi-position measurement technique. The measurement uncertainty of the positions is $\pm 2.5^{\circ}$.

\begin{tabular}{|c|c|c|}
\hline Position & $\boldsymbol{\Phi}$ & $\boldsymbol{\delta}$ \\
\hline 1 & $90^{\circ}$ & $0^{\text {o }}$ \\
\hline 2 & $60^{\circ}$ & $0^{\text {o }}$ \\
\hline 3 & $30^{\circ}$ & $0^{\text {o }}$ \\
\hline
\end{tabular}


proportioner meter. To evaluate $d E / d \theta$, a directional calibration was performed by orienting the probe at different angles to the mean flow. The co-ordinate system for the probe-orientation is explained in Figure 4.6. An orientation of $\Phi=90^{\circ}$ corresponds to a wire normal to the flow. For the current measurements, values of $\Phi=90^{\circ}, 60^{\circ}$ and $45^{\circ}$ were chosen for the directional orientations (see Table 4.5). The wires were calibrated by varying the angle from $\Phi=30^{\circ}$ to $150^{\circ}$ in steps of $5^{\circ}$. Once the calibration data was obtained, the sensitivities were evaluated by using TableCurve3D software (SYSTAT Inc.). Figure 4.9 shows the calibration $3 \mathrm{D}$ surfaces based on the calibration data for a $\mathrm{SN}$ wire. The bridge voltage $(E)$ was plotted as a function of the fluid velocity $(U)$ and the mixture density $(\rho)$. A 3-dimensional surface was fitted to the calibration data and it was ensured that fit gave a smooth and continuous surface over the entire operational range. The fitted curve (see Equation 4.28) had a $R^{2}$. value of 0.9977 was chosen so that an analytical derivative of the function could be easily obtained.

$$
E=\frac{a+b U+c \ln \rho+d(\ln \rho)^{2}+e(\ln \rho)^{3}}{1+f U+g U^{2}+h \ln \rho+i(\ln \rho)^{2}}
$$

The velocity and density sensitivities were plotted in Figures 4.10 and 4.11. The analytical derivatives $(d E / d U \& d E / d \rho)$ of Equation 4.28 were evaluated at each operating point to ensure that the wire sensitivities were defined at the operating points. A similar 3D fit was done for the directional calibration data and was shown in Figure 4.13. The constants in Equation 4.28 were provided in Appendix D. Figure 4.12 shows the errors in bridge voltage (\%). Figures $4.14-4.18$ shows the calibration curves at the overheat ratio of 1.6 . 


\section{Wire \# 1 Overheat Ratio $=1.9$}

Rank 72 Eqn $1045 z=\left(a+b x+c \mid n y+d(\mid n y)^{2}+e(\mid \ln y)^{3}\right) /\left(1+f x+g x^{2}+h \mid n y+i(\mid n y)^{2}\right)$

$r^{2}=0.99771135$ DF Adj $r^{2}=0.99742124 \quad$ FitStdErr $=0.020107227 \quad$ Fstat $=3923.4514$ $a=1.5975671 \quad b=0.14633769 \quad c=1.3360986 d=0.28300571 \quad e=-0.34066712$ $f=-0.01302054 \mathrm{~g}=0.010817117 \mathrm{~h}=1.1282561 \mathrm{i}=0.56214555$

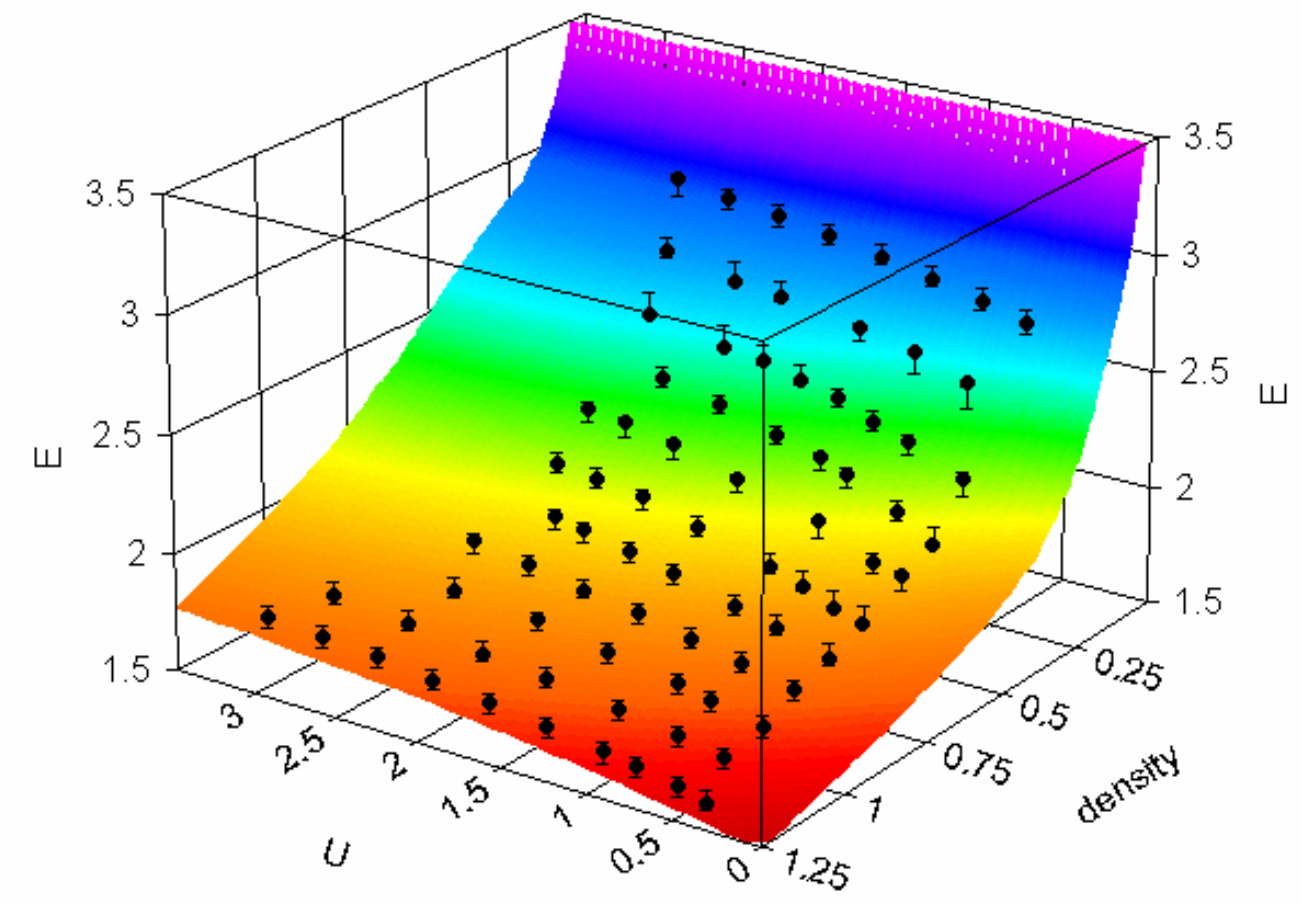

Figure 4.9 Velocity- density calibration for wire 1 at overheat ratio 1.9. 


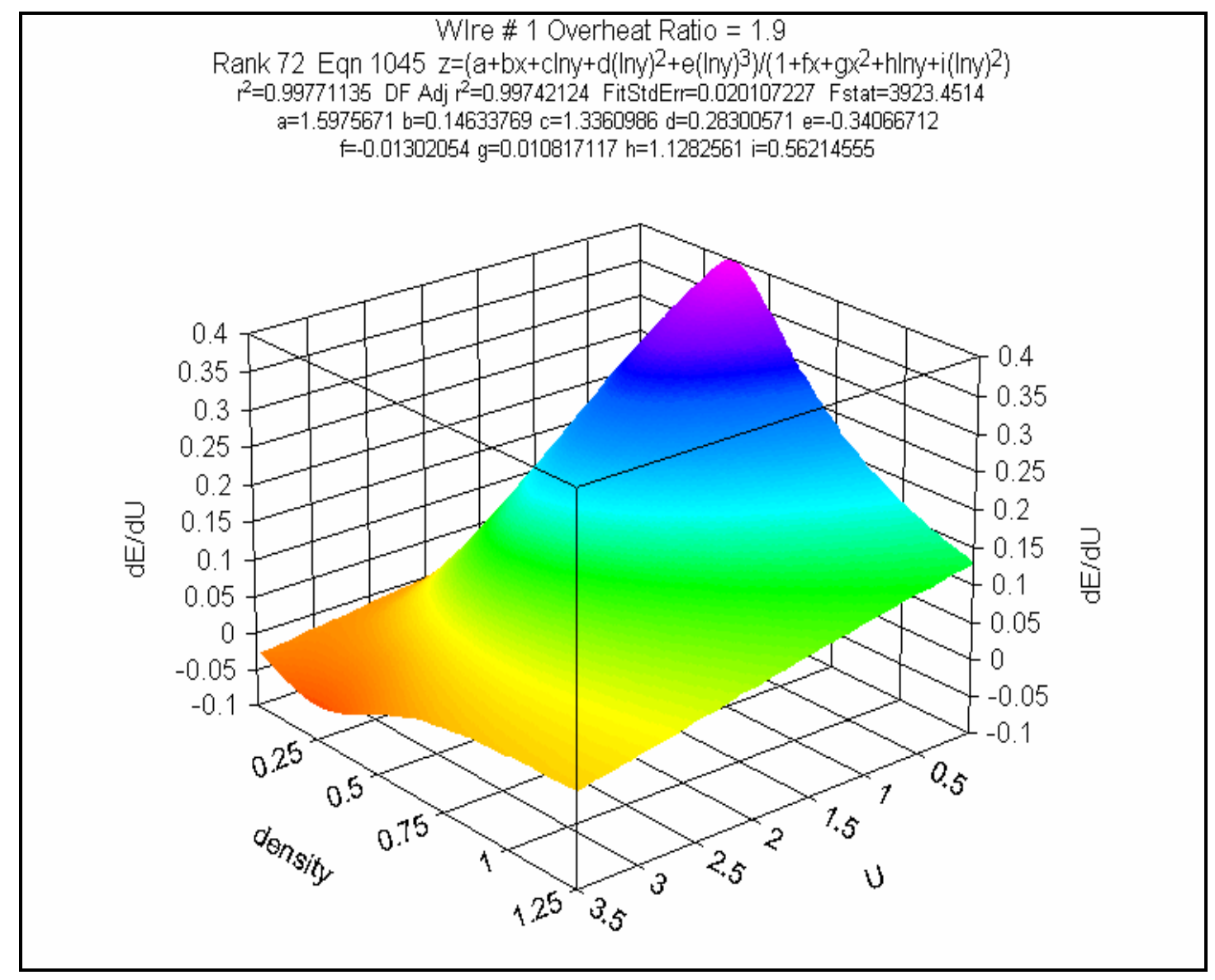

Figure 4.10 $d E / d U$ for wire 1 at overheat ratio 1.9. 
Wire \# 1 Overheat Ratio $=1.9$

Rank 72 Eqn $1045 z=\left(a+b x+c \mid n y+d(\mid n y)^{2}+e(\mid n y)^{3}\right) /\left(1+f x+g x^{2}+h \mid n y+i(\mid n y)^{2}\right)$ $r^{2}=0.99771135$ DF Adj $r^{2}=0.99742124 \quad$ FitStdE $r=0.020107227$ Fstat $=3923.4514$ $a=1.5975671 b=0.14633769 c=1.3360986 d=0.28300571 \quad e=-0.34066712$ $\mathrm{f}=-0.01302054 \mathrm{~g}=0.010817117 \mathrm{~h}=1.1282561 \mathrm{i}=0.56214555$

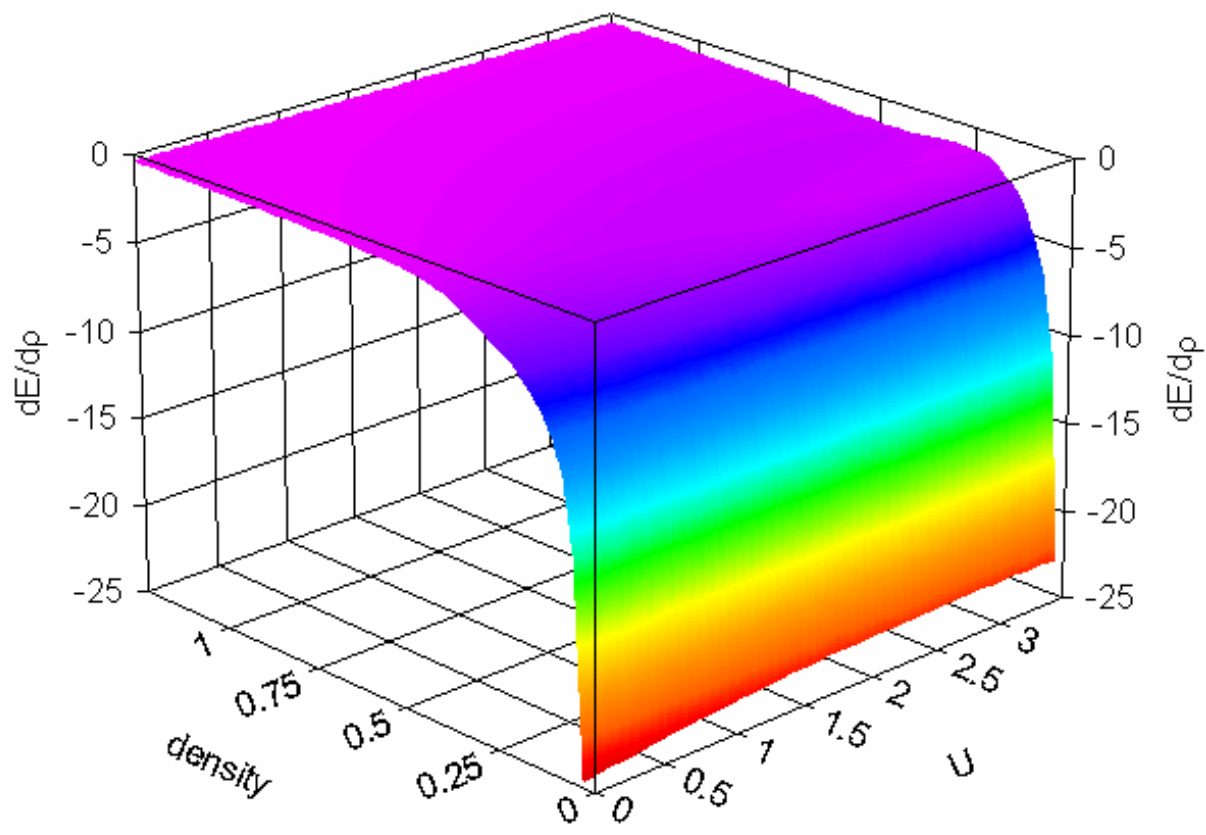

Figure 4.11 $d E / d \rho$ for wire 1 at overheat ratio 1.9. 


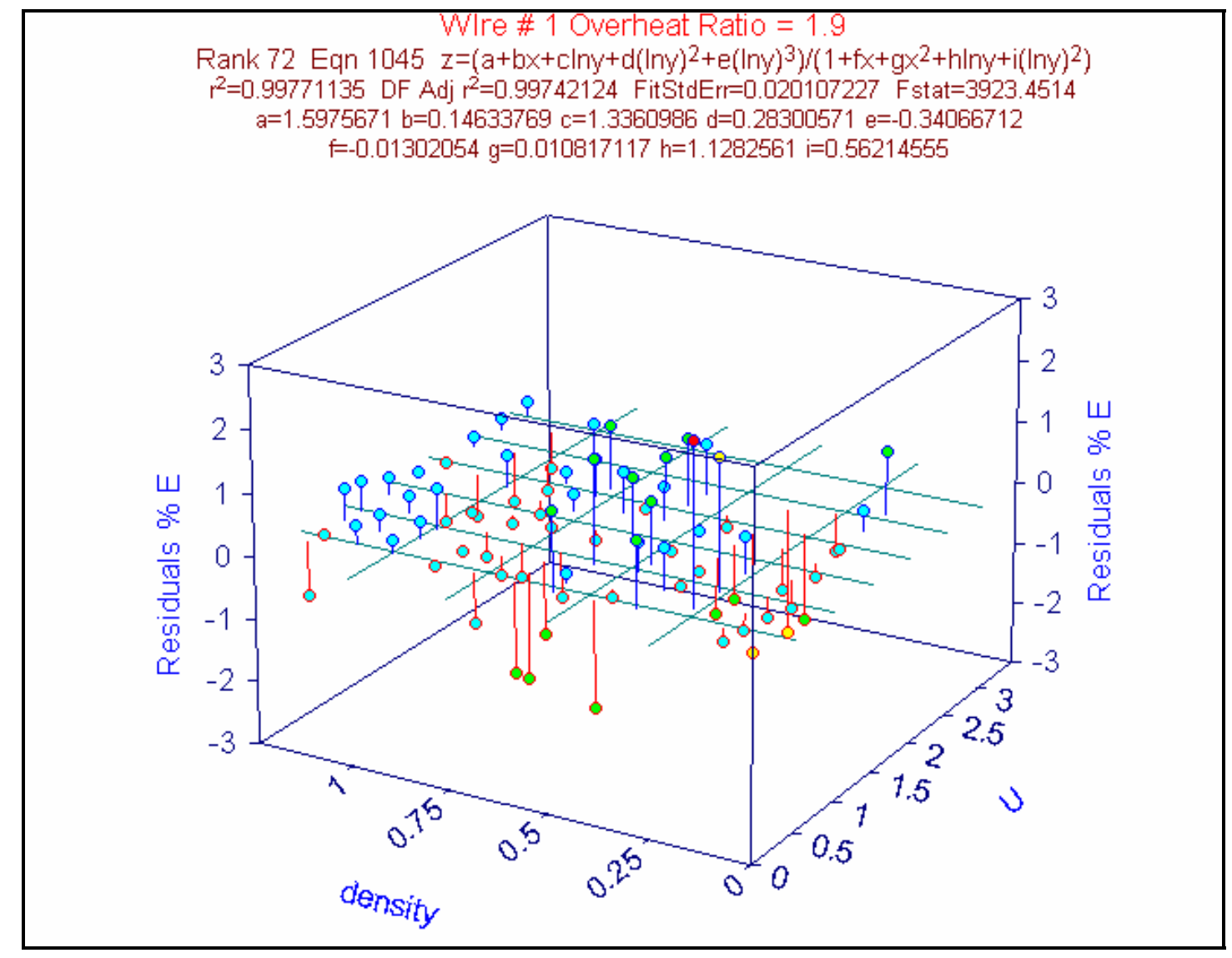

Figure 4.12 Errors in voltage (\%) for wire 1 at overheat ratio 1.9. 


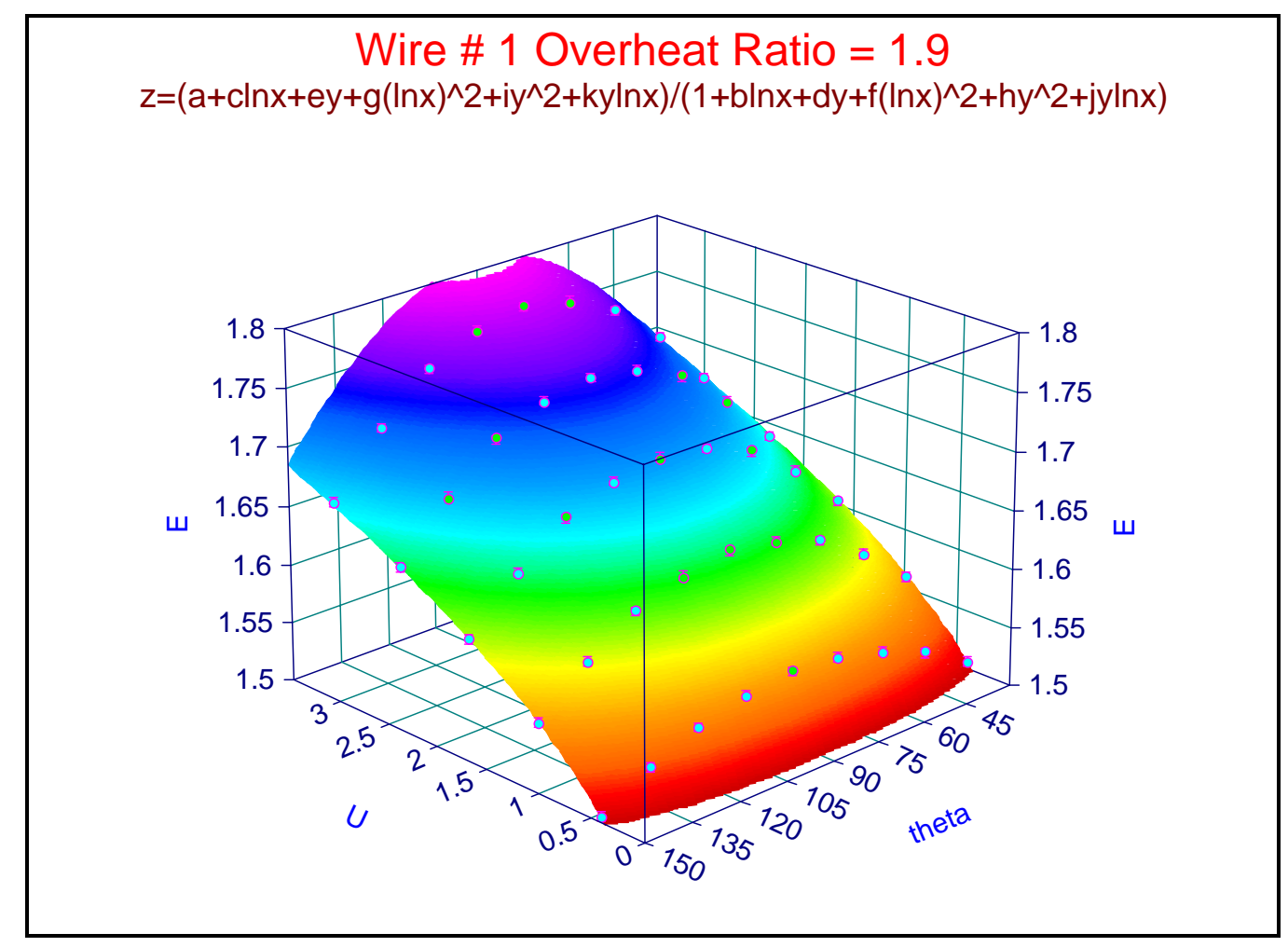

Figure 4.13 Directional calibration for wire 1 at overheat ratio 1.9. 


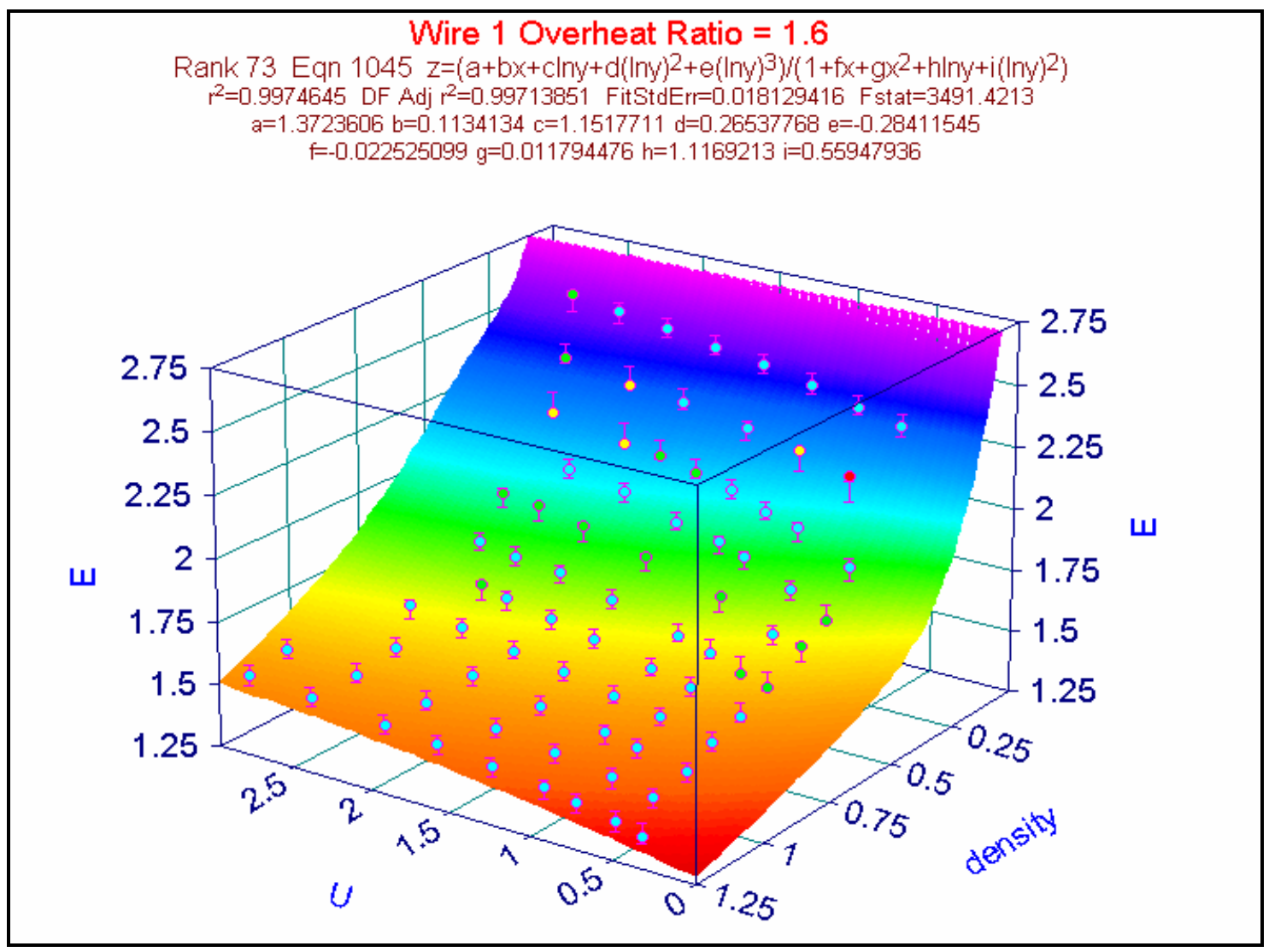

Figure 4.14 Velocity- density calibration for wire 1 at overheat ratio 1.6. 




Figure 4.15 $d E / d U$ for wire 1 at overheat ratio 1.6. 


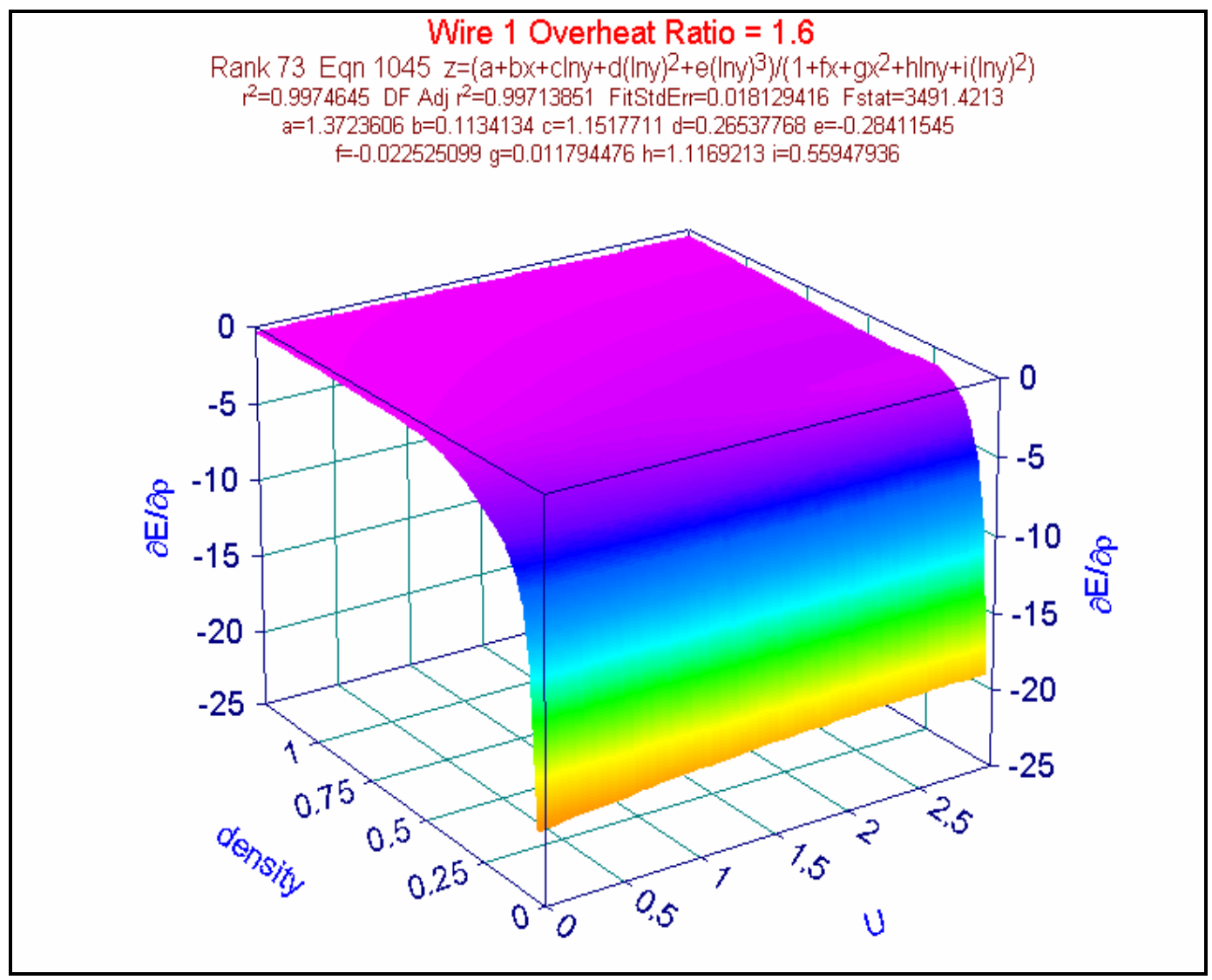

Figure 4.16 $d E / d \rho$ for wire 1 at overheat ratio 1.6. 
Wire 1 Overheat Ratio $=1.6$

Rank 73 Eqn $1045 \mathrm{z}=\left(a+b x+c \mid \ln y+d(\ln y)^{2}+e(\mid \ln y)^{3}\right) /\left(1+f x+g x^{2}+h \mid n y+i(\mid \ln y)^{2}\right)$ $r^{2}=0.9974645$ DF Adj $r^{2}=0.99713851$ FitStdErr $=0.018129416$ Fstat $=3491.4213$ $a=1.3723606 b=0.1134134 c=1.1517711 d=0.26537768 \quad e=-0.28411545$ $f=-0.022525099 \mathrm{~g}=0.011794476 \mathrm{~h}=1.1169213 \mathrm{i}=0.55947936$

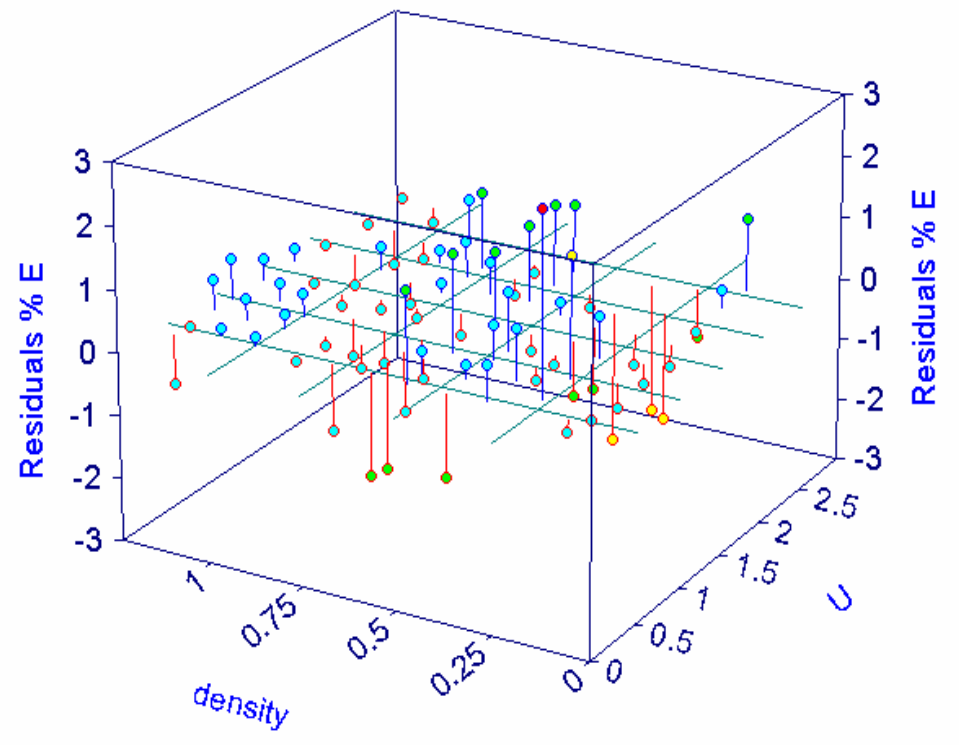

Figure 4.17 Errors in voltage (\%) for wire 1 at overheat ratio 1.6. 
Wire \# 1 Overheat Ratio $=1.6$

$z=\left(a+c \ln x+e y+g(\ln x)^{\wedge} 2+i y^{\wedge} 2+k y \ln x\right) /\left(1+b \ln x+d y+f(\ln x)^{\wedge} 2+h y^{\wedge} 2+j y \ln x\right)$

ш

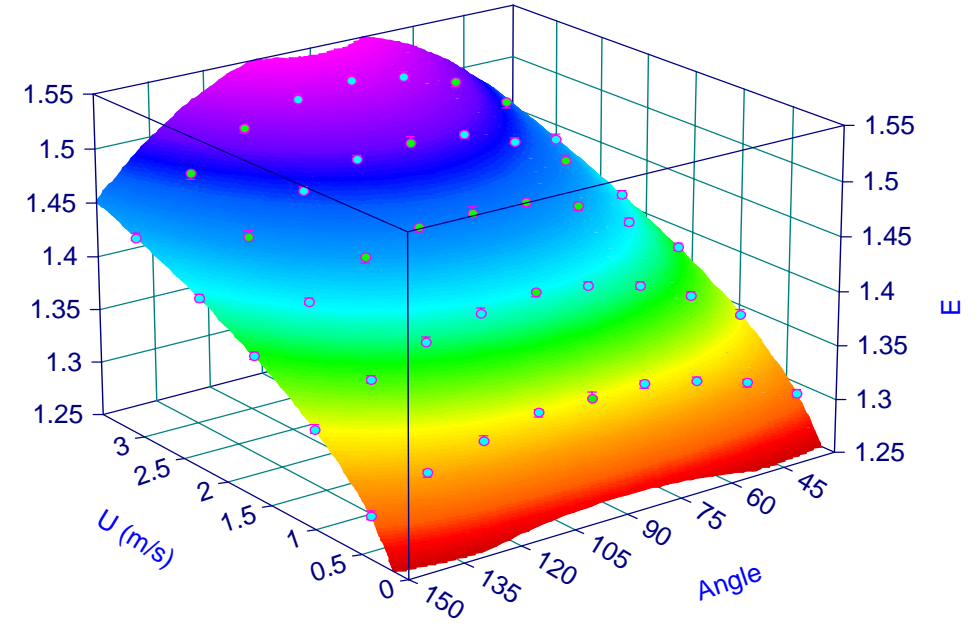

Figure 4.18 Directional calibration for wire 1 at overheat ratio 1.6. 


\section{CONVECTION CORRELATIONS FOR A HEATED WIRE}

\subsection{Heat Transfer Correlations from Heated Wires at Low Reynolds Number}

The use of a hot-wire probe for measuring velocity fluctuations is dependent on laws governing convective heat transfer from a cylinder. These laws are generally too complicated to allow an exact theoretical evaluation for a given fluid velocity. A relation was thus sought to experimentally determine a correlation using similarity principles (Baid 1967; Collis \& Williams 1959; Corrisin 1949). Heat transfer from a wire at a constant temperature is usually by natural convection to the fluid, conduction to the fluid and also to the supports/prongs, and by radiation with the surroundings. However, for the temperature and pressures involved in the present work $\left(T_{\text {wire }}<300^{\circ} \mathrm{C}\right.$ and $\mathrm{P} \sim 1$ atm), radiation effects in heat transfer to the wire was neglected (Hinze 1959). A dimensional analysis on the equations governing convection heat transfer by an incompressible fluid (Comte-Bellot 1976) showed that the dimensionless heat transfer coefficient or Nusselt Number, $\mathrm{Nu}$, for an electrically heated hot-wire has the general form:

$$
N u=N u(\operatorname{Re}, G r, K n, \operatorname{Pr}, \Theta)
$$

where the parameters involved are as follows:

$$
\begin{array}{ll}
\mathrm{Nu} & : \text { Nusselt number based on wire diameter }\left(=h d_{\text {wire }} / k\right) ; \\
\operatorname{Re} & : \text { Reynolds number based on wire diameter }\left(=\rho U d_{\text {wire }} / \mu\right) ; \\
\mathrm{Gr} & : \text { Grashof number based on wire diameter }\left(=(\Delta T / T) g d_{\text {wire }}^{3} / v^{2}\right) ;
\end{array}
$$




$$
\begin{array}{ll}
\operatorname{Pr} & : \text { Prandtl number of the fluid }\left(=\mu C_{p} / k\right) ; \\
K n & : \text { Knudsen number of the fluid }\left(=\lambda / d_{\text {wire }}\right) ; \\
\Theta & : \text { Overheat Ratio }=\left(T_{\text {wire }}-T_{\text {fluid }}\right) / T_{\text {fluid }} ;
\end{array}
$$

King (1914) was perhaps the first to carry out both a rigorous theoretical treatment and a detailed experimental investigation on the heat loss from platinum anemometer wires. He used a whirling arm in air and showed that the heat loss was given by:

$$
N u=A+B \operatorname{Re}^{0.5}
$$

where, $A$ and $B$ were constants which depend on the temperature and dimensions of the wire. The properties of the fluids were evaluated at the free stream temperature. King obtained an analytical solution to the problem by assuming potential flow across the cylinder. However, King's experiments were subject to interference from draughts, both natural and induced and the results show fairly large systematic errors (Collis \& Williams 1959). Forced convection results have been collected and correlated over a wide range of Reynolds numbers (McAdams 1954b). A correlation was suggested for a wide range of Reynolds number $(0.1<R e<1000)$ as follows:

$$
N u=0.32+0.43 \operatorname{Re}^{0.52}
$$

In Equation 5.3, the density $(\rho)$ was evaluated at the free stream temperature while thermal conductivity $(k)$ and absolute/dynamic viscosity $(\mu)$ was evaluated at the mean film temperature $\left(T_{\text {film }}=\frac{T_{\text {wire }}+T_{\text {fluid }}}{2}\right)$. Collis and Williams (1959) analyzed all the available data and proposed a correlation for the range of $0.02<R e<140$. 


$$
N u\left(\frac{T_{\text {film }}}{T_{\text {fluid }}}\right)^{-0.17}=A+B \operatorname{Re}^{n}
$$

Collis and Williams (1959) have shown that molecular effects reduce the heat transfer from very fine wires below the continuum value by an amount which can be estimated by assuming that the temperature difference was reduced by a temperature jump which can be pre-determined from Kinetic Theory. The values of $n, A$ and $B$ can be found in Table 5.1. As may be seen from the table, Collis and Williams propose a linear dependence of the Nusselt number with a 0.45 power of Reynolds number for $R e<44$. For higher values of Re, they use a 0.51 power of Reynolds number. Their results were consistent as they show the same form of interdependence as previous correlations.

It should be noted that the law of convective heat transfer from a wire at a uniform temperature will be modified because of non-uniform wire temperature arising from end cooling (conduction end losses). There has been extensive work computing theoretical temperature distributions with end cooling and correction procedures have been devised to account for these effects (Aihara et al. 1967; King 1914; Preckshot \& Gorman 1958). However such correlations are difficult to apply and are not used in most calibrations (Andrews et al. 1972). Earlier findings in air (Collis \& William 1959) reveals that the major effect of the end cooling in the apparent value of $N u$, was to alter the constants $A$ and $B$ in Equation 5.4 but not the power of Re. In various experiments done with hotwires, the wire diameter was sufficiently small for the effects of molecular nature of the fluid to be experienced. Molecular effects take the form of changed boundary conditions as compared with continuum flow - there is a jump in temperature between the surface 
of the wire and the fluid adjacent to it (Collis \& Williams 1959). The fluid slips or moves over the hot-wire surface with a finite velocity. In rarefied gas dynamics, the ratio of mean free path to wire diameter, which was defined as the Knudsen Number $(K n)$ takes into account the molecular effects. However, there was still insufficient knowledge about all the parameters defined in Equation 5.1. Formulation of a correlation using all these parameters was impractical and thus various assumptions were made. Free and forced convections have been treated separately (Collis \& Williams 1959).

Considerable work has been reported on free convection from cylinders (Cole \& Roshko 1954; Collis \& Williams 1959; Van der Hegge Zijnen 1956). The correlation takes the form of

$$
N u=N u(G r, \operatorname{Pr}, \Theta)
$$

A theoretical solution with a boundary layer approximation was provided (McAdams 1954). Van der Hegge Zijnen (1956) reviewed all available data and proposed a correlation (see Table 5.1). The physical properties were evaluated at the mean film temperature. This correlation fits very well with other correlations (Cole \& Roshko 1954). Relatively few studies have been made to analyze the combined effects of free and forced convection. Van der Hegge Zijnen (1956) suggested combining the two types of heat transfer vectorially. Subsequently, more fundamental analysis to the problem has been made (Acrivos 1966; Chen \& Mucoglu 1975; Gebhart \& Pera 1970) and the findings were summarized as follows:

(a) The two effects were non-additive. 
TABLE 5.1 Convection heat transfer correlations for flow over a heated cylinder

\begin{tabular}{|c|c|c|c|c|}
\hline Author & Mode of Convection & Correlation Equation & $K n$ & $\mathbf{R e}$ \\
\hline King & Forced & $N u=0.318+0.69 \mathrm{Re}^{0.50}$ & $<0.01$ & $0.055-55$ \\
\hline Hilpert & Forced & $N u=0.891+\left[\operatorname{Re}\left(T_{w} / T_{a m b}\right)^{0.25}\right]^{0.33}$ & $<0.005$ & $1-4$ \\
\hline McAdams & Forced & $N u=0.32+0.43 \mathrm{Re}^{0.52}$ & & $0.1-10^{3}$ \\
\hline V. D. H. Zijnen & $\begin{array}{c}\text { Forced } \\
\text { Free }\end{array}$ & $\begin{array}{l}N u=0.38 \operatorname{Pr}^{0.2}+\left(0.56 \mathrm{Re}^{0.50}+0.001 \mathrm{Re}\right) \operatorname{Pr}^{0.33} \\
N u=0.35+0.25(G r \cdot \operatorname{Pr})^{1 / 8}+0.45(G r \cdot \operatorname{Pr})^{1 / 4}\end{array}$ & $<<0.01$ & $0.01-10^{4}$ \\
\hline Collis \& Williams & Forced & $\begin{aligned} N u\left(T_{m} / T_{\text {amb }}\right)^{-0.17} & =0.24+0.56 \operatorname{Re}^{0.45} \\
& =\quad 0.48 \operatorname{Re}^{0.51}\end{aligned}$ & $\begin{array}{c}0.001- \\
0.03\end{array}$ & $\begin{array}{r}0.02-44 \\
44-140\end{array}$ \\
\hline Churchill \& Brier & Forced & $N u=0.535 \operatorname{Re}^{0.50}\left(T_{w} / T_{a m b}\right)^{0.12}$ & & $300-2300$ \\
\hline Davies and Fisher & Combined & $N u=(2.6 / \gamma \pi) \operatorname{Re}^{0.33}$ & $<0.01$ & $0-50$ \\
\hline Parnas & Forced & $N u=0.823 \operatorname{Re}^{0.50}\left(T_{w} / T_{a m b}\right)^{0.085}$ & 0.0015 & $10-60$ \\
\hline Ahmad & Forced & $N u\left(v_{a m b} / v_{m}\right)^{0.15}=0.21+0.497 \mathrm{Re}^{0.45}$ & $<0.01$ & $4-40$ \\
\hline Andrews et al. & Combined & $N u_{c}=0.34+0.65 \mathrm{Re}^{0.45}$ & $\begin{array}{c}0.01- \\
0.03\end{array}$ & $0.015-20$ \\
\hline
\end{tabular}


TABLE 5.2 List of convection heat transfer correlations involving hot-wire anemometry (small cylinders)

\begin{tabular}{|c|c|c|c|c|c|c|}
\hline Author & $T_{\text {fluid }}$ & $\begin{array}{c}l / d \\
\text { ratio }\end{array}$ & Correlation Equation & $T_{\mathrm{w}} / T_{\mathrm{amb}}$ & $K n$ & $\mathbf{R e}$ \\
\hline King & $\begin{array}{l}\text { Independent } \\
\text { of } T\end{array}$ & $>1000$ & $N u=0.318+0.69 \mathrm{Re}^{0.50}$ & $1.5-4.50$ & $<0.01$ & $\begin{array}{c}0.055- \\
55\end{array}$ \\
\hline Hilpert & $T_{\mathrm{m}}$ & $\begin{array}{l}539- \\
5130 \\
\end{array}$ & $N u=0.891+\left[\operatorname{Re}\left(T_{w} / T_{a m b}\right)^{0.25}\right]^{0.33}$ & $1.14-4.5$ & $<0.005$ & $1-4$ \\
\hline $\begin{array}{l}\text { V. D. H. } \\
\text { Zijnen }\end{array}$ & $T_{\mathrm{m}}$ & 553 & $N u=0.38 \operatorname{Pr}^{0.2}+\left(0.56 \operatorname{Re}^{0.50}+0.001 \mathrm{Re}\right) \operatorname{Pr}^{0.33}$ & 1.34 & $<<0.01$ & $\begin{array}{c}0.01- \\
10^{4} \\
\end{array}$ \\
\hline $\begin{array}{l}\text { Collis \& } \\
\text { Williams }\end{array}$ & $T_{\mathrm{m}}$ & $\begin{array}{c}2070- \\
8660\end{array}$ & $\begin{aligned} N u\left(T_{m} / T_{\text {amb }}\right)^{-0.17} & =0.24+0.56 \operatorname{Re}^{0.45} \\
& =0.48 \operatorname{Re}^{0.51}\end{aligned}$ & $1.1-2$ & $\begin{array}{l}0.001- \\
0.03\end{array}$ & $\begin{array}{l}0.02-44 \\
44- \\
140\end{array}$ \\
\hline $\begin{array}{l}\text { Davies and } \\
\text { Fisher } \\
\end{array}$ & $T_{\mathrm{amb}}$ & $\begin{array}{l}400- \\
1200 \\
\end{array}$ & $N u=(2.6 / \gamma \pi) \operatorname{Re}^{0.33}$ & $<2$ & $<0.01$ & $0-50$ \\
\hline Parnas & $T_{\mathrm{amb}}$ & 1000 & $N u=0.823 \operatorname{Re}^{0.50}\left(T_{w} / T_{a m b}\right)^{0.085}$ & $1.2-3.5$ & 0.0015 & $10-60$ \\
\hline Andrews et al. & $T_{\mathrm{m}}$ & $>400$ & $N u_{c}=0.34+0.65 \mathrm{Re}^{0.45}$ & $1.05-3.5$ & $\begin{array}{c}0.01- \\
0.03 \\
\end{array}$ & $\begin{array}{c}0.015- \\
20 \\
\end{array}$ \\
\hline Present Work & $T_{\mathrm{m}}$ & 250 & $N u=0.2719+0.0 .3763 \mathrm{Re}^{0.50}$ & $1.4-1.9$ & 0.01 & $0.1-2$ \\
\hline
\end{tabular}


(b) The transition from one effect to the other was a gradual one and was guided by the parameter: $G r / \operatorname{Re}^{2}$. However because of the gradual shift from one mode to the other, it was very difficult to predict the value of the parameter at which each convection mode would dominate.

Collis and Williams (1959) chose the transition point as the regime where $N u$ for mixed convection equals that for pure convection at the same $G r$. The generally accepted conclusion was that buoyancy effects were negligible for $\operatorname{Re}>G r^{1 / 3}$. Table 5.1 provides an assortment of correlations available over the entire regime (free to forced via mixed convection), An excellent review work on heat transfer correlations was provided by Andrews et al. (1972). A review of the heat transfer correlations used in hot-wire anemometry was provided in Table 5.2. Various parameters critical to measurement like overheat ratio, $l / d$ ratio, $K n, R e$ were tabulated.

\subsection{Behavior of Hot-Wire/Film in Gas Mixtures}

Hot-wire anemometry has been used in the last 50 years to measure instantaneous velocity and species concentration in a flow (Corrisin 1949). The basis for this measurement is the use of a modified form of Equation 5.4 (Collis \& William 1959) which is written in the form:

$$
N u\left(\frac{T_{\text {film }}}{T_{\text {fluid }}}\right)^{-0.17}=A\left(\frac{\operatorname{Pr}_{\text {mix }}}{\operatorname{Pr}_{\text {air }}}\right)^{0.2}+B\left(\frac{\operatorname{Pr}_{\text {mix }}}{\operatorname{Pr}_{\text {air }}}\right)^{0.33} \mathrm{Re}^{n}
$$

All the properties are evaluated at the mean film temperature $T_{\text {film. }}$. Equation 5.5 indicates that the rate of heat transfer is dependent on the properties and the velocity of the fluid. 
Thus, if the exact composition of the mixture passing by the wire was known, the velocity can be estimated. The technique was to use two probes (combinations of hot film and hot-wire) at two different temperatures (or overheat ratios) close to each other which provides two signals simultaneously for measuring velocity and concentration simultaneously (Simpson \& Wyatt 1973; Way \& Libby 1970; 1971). This arrangement has been used for measure the turbulence structure of a helium jet in air (Way \& Libby 1970; 1971). Simpson and Wyatt (1973) reported behavior of hot film probes in heliumair and argon-air mixtures. Wasan and Baid (Wasan \& Baid 1971) have performed extensive validation checks of the modified Collis and Williams equation in a AirCarbon-dioxide mixture. We thus decided to formulate a correlation in the form of Equation 5.4 to predict the heat transfer from a hot-wire (cylinder) for a binary gas mixture of air and helium. The correlation takes into account effects of thermal slip (Wu \& Libby 1971) as we fit direct calibration data into the correlation by taking into account errors arising from these effects.

\subsection{Effect of Temperature Jump on the Heat Transfer Coefficient}

The use of small diameter wires $\left(d_{\text {wire }} \leq 5 \mu \mathrm{m}\right)$ in hot-wire anemometry means that non-continuum effects cannot be ignored even at a pressure of one atmosphere (Andrews et al. 1972). There is a discontinuity in the temperature distribution at the wall when heat transfer (by conduction) takes place between a rarefied gas and the heated bounding wall (the wire in this case). Assuming that $T_{\text {wire }}$ as the wall temperature and $T_{\mathrm{s}}$ as the gas temperature if the temperature gradient remained unchanged up to the surface, the discontinuity or temperature jump at the interface is given by: 


$$
T_{\text {wire }}-T_{s}=-\xi \frac{\partial T}{\partial r}
$$

where $\xi$ is a constant and has dimensions of length and is known as the temperature jump distance. Kennard (1938) derives an expression for $\xi$ in terms of the properties of the gas and the surface as:

$$
\xi=\frac{2-\chi}{\chi} \frac{2 \gamma}{\gamma+1} \frac{\lambda}{\operatorname{Pr}}
$$

where $\chi$ is the thermal accommodation coefficient of the wire surface for a particular gas; $\gamma=C_{p} / C_{v}$ is the ratio of specific heats; and $\lambda$ is the mean free-path of the gas molecule. The accommodation coefficient is defined as the fractional extent to which molecules which falls on the surface, and are reflected or re-emitted, have their mean energy adjusted (or "accommodated") towards the values which it would obtain if the returning molecules are to issue as a stream from a mass of gas at the wall temperature (Andrews et al. 1972). For most gas-surface combinations, $\xi$ is of the same order as $\lambda$. A slip parameter $\beta$ (Kassoy 1967) is given by:

$$
\beta=\frac{\xi}{d_{\text {wire }}}=\frac{2-\chi}{\chi} \frac{2 \gamma}{\gamma+1} \frac{K n}{\operatorname{Pr}}
$$

Equation 5.7 is a first order approximation which is valid if the velocity and temperature profiles are linear over a distance equal to the mean free path of the gas molecules (Deissler 1964). However at high values of Knudsen number, the profiles are significantly non-linear and this assumption is no longer valid (Andrews et al. 1972). However for the present work, the values of Knudsen number are small $(<0.1)$ and the 
use of a linear approximation is justified. The heat flux $q^{\prime \prime}$ from the wire can be written as follows:

$$
q^{\prime \prime}=h_{s}\left(T_{s}-T_{g}\right)=h_{w}\left(T_{\text {wire }}-T_{g}\right)
$$

Equation 5.9a can be re-written as:

$$
h_{s}=h_{w}\left(1+\frac{T_{\text {wire }}-T_{s}}{T_{s}-T_{g}}\right)
$$

The heat loss by conduction from the wire can be written as:

$$
q^{\prime \prime}=-\left.k\left(\frac{\partial T}{\partial r}\right)\right|_{T=T_{s}}
$$

Equations 5.6, 5.9a and 5.9c, we can be combined to get:

$$
\frac{T_{\text {wire }}-T_{s}}{T_{s}-T_{g}}=\frac{\xi_{T_{s}} h_{s}}{k_{T_{s}}}
$$

The Nusselt number $N u$ is defined as $N u=h_{w} d_{\text {wire }} / k_{T_{\text {fllm }}}$ and the continuum Nusselt number $N u_{\mathrm{c}}$ is defined as $N u_{c}=h_{s} d_{\text {wire }} / k_{T_{\text {film }}}$. Combining Equations $5.9 \mathrm{~b}$ and 5.10 with the definition of $N u$ and $N u_{\mathrm{c}}$, we get:

$$
N u_{c}=\frac{N u}{1-N u \frac{\xi_{T_{s}} k_{T_{\text {flim }}}}{k_{T_{s}} d_{\text {wire }}}}
$$

For a perfect gas at constant pressure, given that: $K n \propto T^{0.5+x}, \mu \propto T^{x}$ and $k \propto T^{y}$ (Andrews et al. 1972), where $x$ is the power dependence of $T$ for viscosity and $y$ is the power dependence of $T$ for thermal conductivity $(x=0.68 ; y=0.8)$. Equation 5.11 can thus be written as: 


$$
N u_{c}=\frac{N u}{1-\sigma K n N u}
$$

$K n$ is evaluated at $T_{f i l m}$ and $\sigma$ was given by:

$$
\sigma=\left.\frac{2-\chi}{\chi} \frac{2 \gamma}{\gamma+1} \frac{1}{\operatorname{Pr}}\right|_{T_{s}}\left(\frac{T_{s}}{T_{\text {film }}}\right)^{0.5+x-y}
$$

A major hindrance in the use of Equation 5.13 was the determination of the thermal accommodation coefficient $\chi$. A number of reviews indicate that there is a no agreement of the values of $\chi$ for a given metal-gas interface. The value of $\chi$ is a strong function of the properties of the wire surface and varied by a factor of 10 between a clean surface and one which was contaminated (Andrews et al. 1972; Kaminsky 1965). Andrews et al. (1972) lists a comparison of accommodation coefficients of different gas-metal combinations. For the present study, the SN wire probes used were made of platinum plated tungsten (Dantec Dynamics). So we choose an accommodation coefficient of 0.02 for $\mathrm{He}-\mathrm{Pt} / \mathrm{W}$ interface and 0.90 for the air-Pt/W interface based on values available in the literature (Andrews et al. 1972).

\subsection{Convective Correlations for Binary Air-Helium Mixture}

The identification of a correct non-dimensional relationship for hot-wire probes is complicated because of the following factors:

- The values of fluid properties $\rho, \mu$ and $k$ vary with temperature and must be evaluated at a suitable reference temperature. The choice of this reference temperature is not well defined. A popular choice is the mean film temperature $T_{\text {film }}=\left(T_{\text {wire }}+T_{\text {fluid }}\right) / 2$. 
- The heat transfer relationship corresponds to voltage drop across the wire, $E_{\mathrm{w}}$. Details of the Wheatstone-bridge (CTA) circuit are essential to relate $E_{\mathrm{w}}$ to the anemometer output voltage $E$. The wire properties $R_{\mathrm{w}}$ and $\alpha$ must be known accurately.

For the Nusselt number to be calculated, the heat loss by conduction from the wire must be known. The electrical energy supplied to a hot-wire is dissipated by convection and conduction to the prongs. An accurate estimate of conduction heat loss requires knowledge of the temperature gradient of the wire at the supports which is difficult to obtain. For calibration / correlation purposes, an absolute value of Nusselt number is not required. The need is to formulate a correlation which will hold under experimental conditions. It was thus a common practice to correlate the Reynolds number with an apparent Nusselt number (Andrews et al. 1972). The results of the calibration are presented in the form:

$$
N u_{c}=A+B \operatorname{Re}^{0.5}
$$

Figure 5.1 shows that $E$ is best correlated to $U$ by a relationship as given by King's law in which the exponent $n=0.5$. Collis and Williams (1959) used an exponent of 0.45 . However, their data was fitted for a large Reynolds number range. The calibration was performed in a facility described in section 4.1.1. It is seen that the response is fairly 


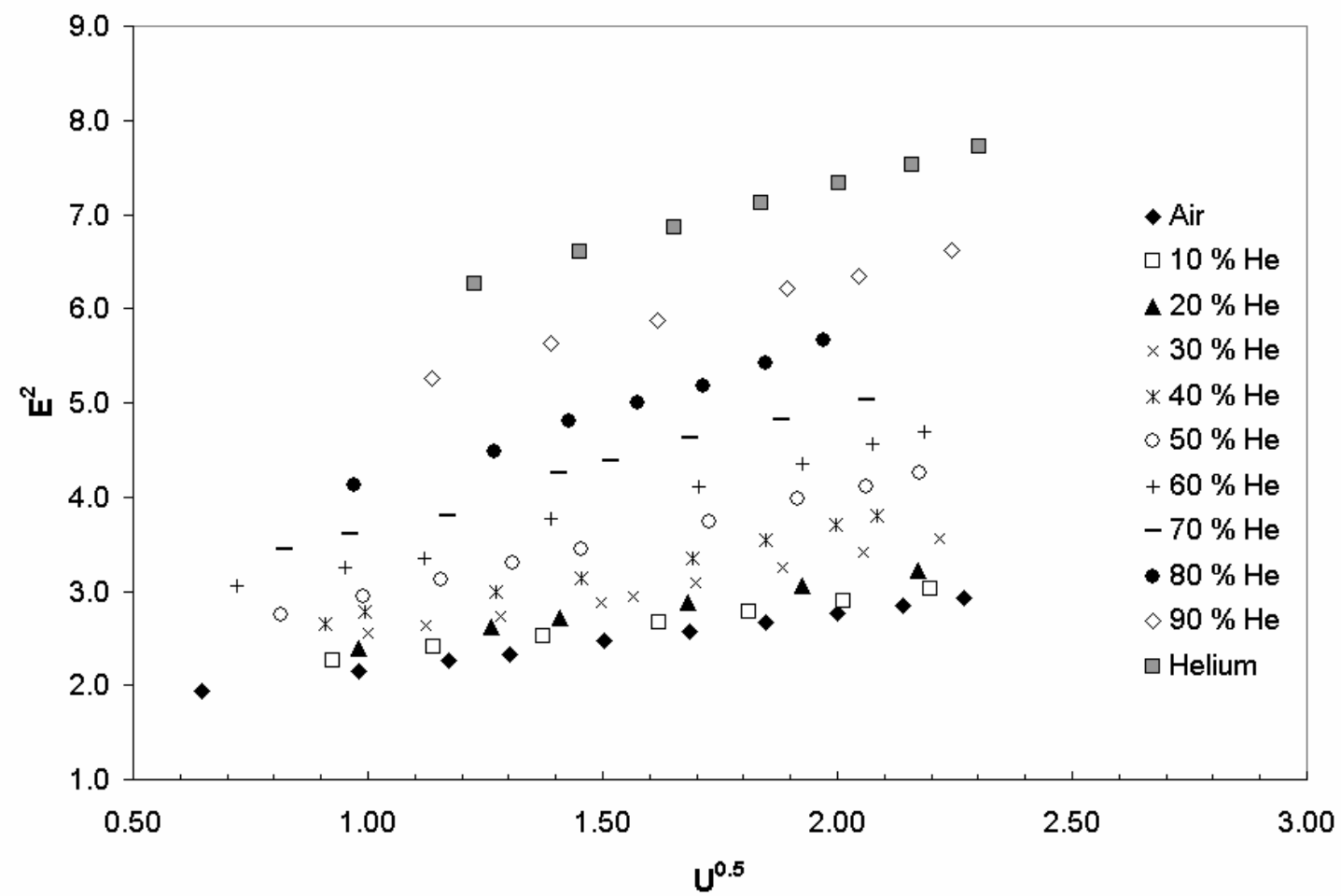

Figure 5.1 Hot-wire calibrations in different volume fractions of helium in a binary airhelium mixture. (Note: Uncertainty in voltage measurements is $0.5 \%$.) 
linear over the entire velocity range. However, as the volume fraction of helium in the flow increases, the slope increases.

\subsubsection{Properties of Gas Mixtures}

The properties necessary for calculating the heat transfer correlations are evaluated based on a binary gas mixture with one component as air and the second as helium. The density is determined from thermodynamic principles and is a linear combination of the mole (volume) fraction of the components (1: air, 2: helium).

$$
\rho_{\text {mix }}=\rho_{1} x_{1}+\rho_{2} x_{2}
$$

The viscosity of the mixture is determined from a method by Wilke (1950) and is one of the more accurate methods of estimation of viscosities of gas mixtures. The viscosity of the mixture is defined as:

$$
\begin{aligned}
\mu_{\text {mix }}=\mu_{1}\left[1+\frac{x_{2}}{x_{1}} \Phi_{12}\right]^{-1}+\mu_{2}\left[1+\frac{x_{1}}{x_{1}} \Phi_{21}\right]^{-1} \\
\text { where, } \quad \Phi_{i j}=\left[1+\left(\frac{\mu_{i}}{\mu_{j}}\right)^{\frac{1}{2}}\left(\frac{M_{j}}{M_{i}}\right)^{\frac{1}{4}}\right]^{2}\left[8\left(1+\frac{M_{i}}{M_{j}}\right)\right]^{\frac{1}{2}}, i \neq j
\end{aligned}
$$

$x_{1}, x_{2}$ are the mole (volume) fraction of the individual components in the mixture; $M_{1}$ and $M_{2}$ are the molecular weights of the pure gases.

The thermal conductivity of the gas mixture is obtained by using a formula by Mason \& Saxena (1958) which is derived from kinetic theory: 

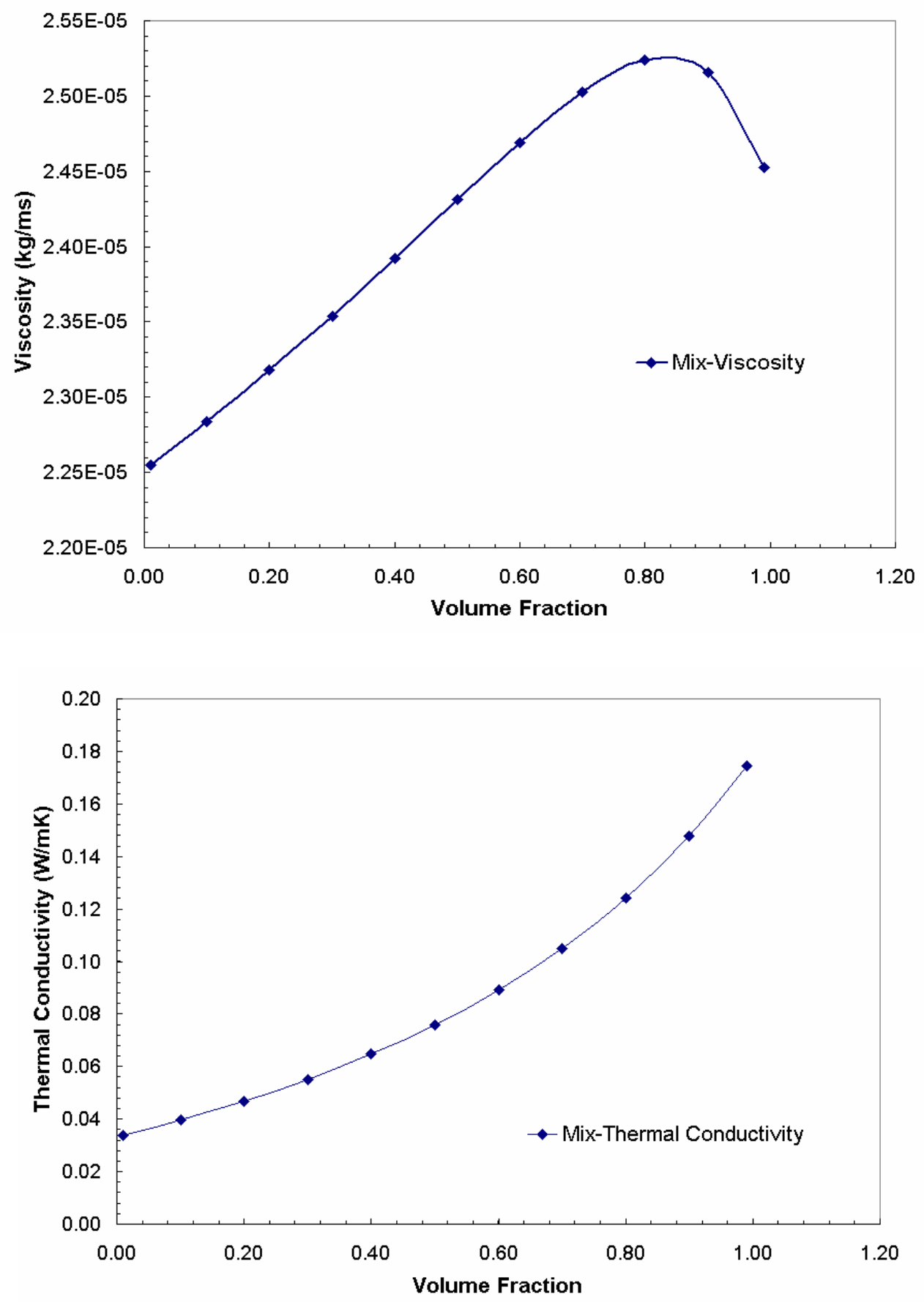

Figure 5.2 Absolute viscosity and thermal conductivity of air-helium mixture. The properties of the pure gases are evaluated at: $T_{\text {film }}=\left(T_{\text {wire }}+T_{\text {fluid }}\right) / 2$. The properties are obtained using theoretical formulations by Wilke(Equation 5.16) and by Mason $\&$ Saxena (Equation 5.17). 


$$
k_{m i x}=k_{1}\left[1+\frac{x_{2}}{x_{1}} \Phi_{12}^{\prime}\right]^{-1}+k_{2}\left[1+\frac{x_{1}}{x_{1}} \Phi_{21}^{\prime}\right]^{-1}
$$

where, $\quad \Phi_{i j}^{\prime}=1.065\left[1+\left(\frac{k_{i} P_{j}}{k_{j} P_{i}}\right)^{\frac{1}{2}}\left(\frac{M_{j}}{M_{i}}\right)^{\frac{1}{4}}\right]^{2}\left[8\left(1+\frac{M_{i}}{M_{j}}\right)\right]^{-\frac{1}{2}}, i \neq j$

and,

$$
P_{i}=0.115+0.354\left(c_{p i} / R_{i}\right) ;
$$

Figure 5.2 shows the variation in viscosity and thermal conductivity for different volume fractions of helium.

The specific heat at constant volume $\left(C_{\mathrm{v}}\right)$ is determined from thermodynamic principles and is a linear combination of the mole (volume) fraction of the components (1: air, 2: helium).

$$
C_{v, \text { mix }}=C_{v 1} x_{1}+C_{v 2} x_{2}
$$

The specific heat at constant pressure $\left(C_{\mathrm{p}}\right)$ is also determined from thermodynamic principles and is a linear combination of the mass fractions of the components

$$
C_{p, m i x}=C_{p 1} m_{1}+C_{p 2} m_{2}
$$

The values of $C_{\mathrm{p}}$ and $C_{\mathrm{v}}$ used for the 2 gases can be found in Appendix C.

\subsubsection{Effect of Binary Air-Helium Mixture}

The results of calibrations in air at atmospheric pressure for various values of overheat ratio from 1.6 to 1.9 are shown in Figure 5.3. The aspect ratio for the probe $\left(l_{\text {wire }} / d_{\text {wire }}\right)$ was 250 . The results are similar to those by Andrews et al. (1972) and it is observed that by evaluating gas properties at the mean film temperature, $T_{\text {film }}$, a separate 


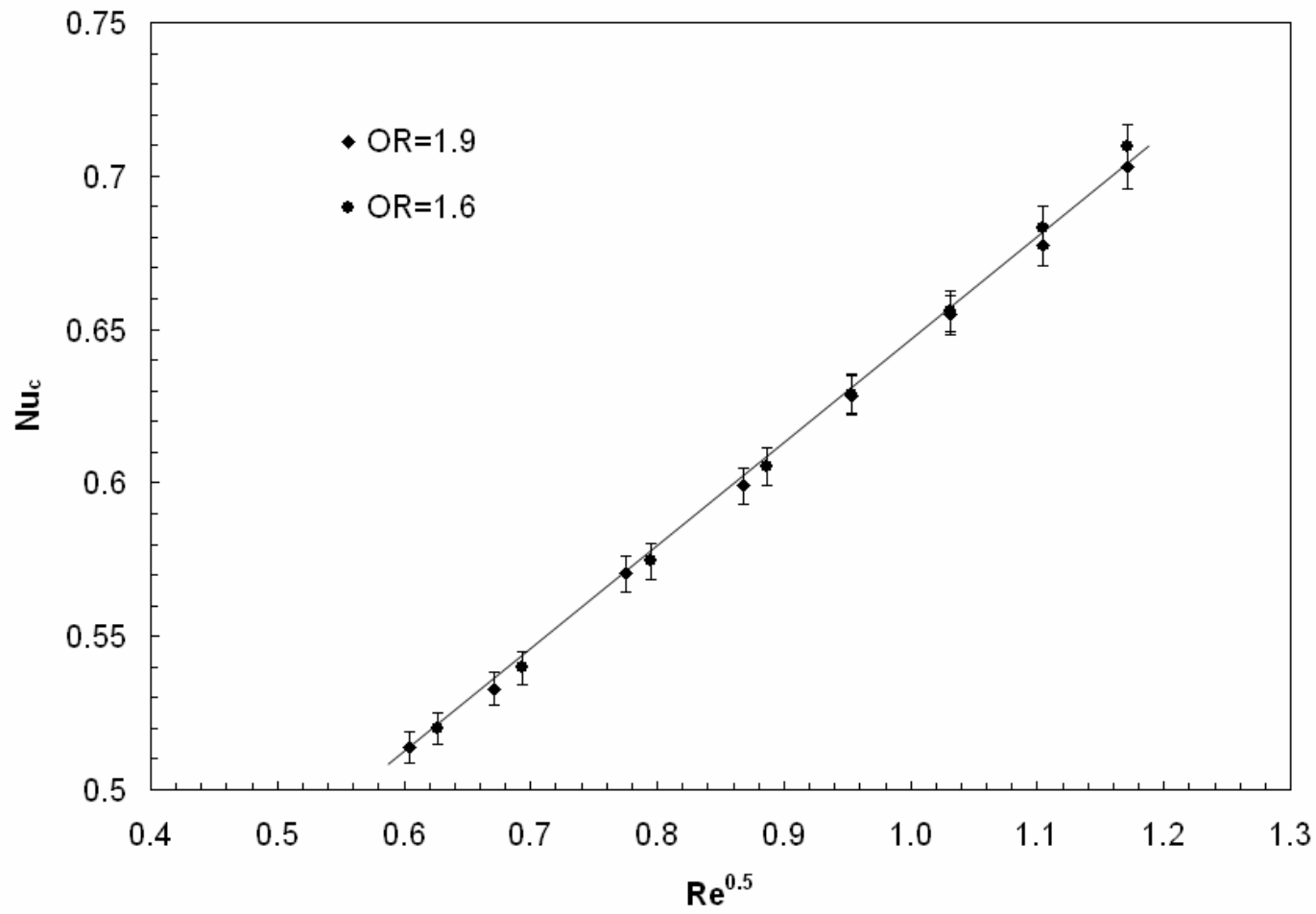

Figure 5.3 Calibration in air at different overheat ratios. 
temperature loading term is not required. From a viewpoint of computing the wire continuum Nusselt Number, Equation 5.14 should be stated in terms of wire properties and the properties of the gas mixture. Thus using a variation of fluid properties (density for our case), a family of curves displaying anemometer output voltage as a function of species concentration was generated as shown in Figure 5.1. The heat transfer from flow over a cylinder is given by:

$$
q^{\prime \prime}=h\left(T_{\text {wire }}-T_{\text {fluid }}\right)=\frac{E_{w}{ }^{2}}{\pi l_{w} d_{w} R_{w}}=\frac{k}{d_{w}} N u\left(T_{\text {wire }}-T_{\text {fluid }}\right)
$$

After the Nusselt number is evaluated, the continuum Nusselt number is calculated as discussed in Section 5.3. Sample calculations for the various parameters are given in Table 5.3. The results are plotted in Figure 5.4. It is seen that the helium-air calibration data for the entire range of volume fraction collapse to a straight line. The form of the calibration is as follows:

$$
N u_{c}=0.2719+0.3763 \mathrm{Re}^{0.5}
$$

Certain points in the lower Reynolds number range tend to show a high degree of scatter. This scatter is because of components of velocity, induced in the heated fluid adjacent to the cylinder by the buoyancy forces. The buoyancy forces are found to become more significant as the proportion of helium in the mixture increases. 
TABLE 5.3 Sample calculations for various correlation parameters for air (at overheat ratio 1.7)

\begin{tabular}{|c|c|}
\hline Parameter & Value \\
\hline$P_{0}$ & $76 \mathrm{~mm}$ of $\mathrm{Hg}=101.3 \mathrm{kPa}$ \\
\hline$T_{\text {wire }}$ & $493.15 \mathrm{~K}$ \\
\hline$\lambda=\frac{R T}{\sqrt{2} \pi d^{2} N_{\text {air }} P}$ & $0.1309 \mu \mathrm{m}$ \\
\hline$K n=\lambda / d$ & 0.0262 \\
\hline$\chi$ & 0.9 \\
\hline$\beta$ & 0.0299 \\
\hline$x$ & 1.9 \\
\hline$y$ & 0.86 \\
\hline$\sigma$ & 3.24 \\
\hline
\end{tabular}




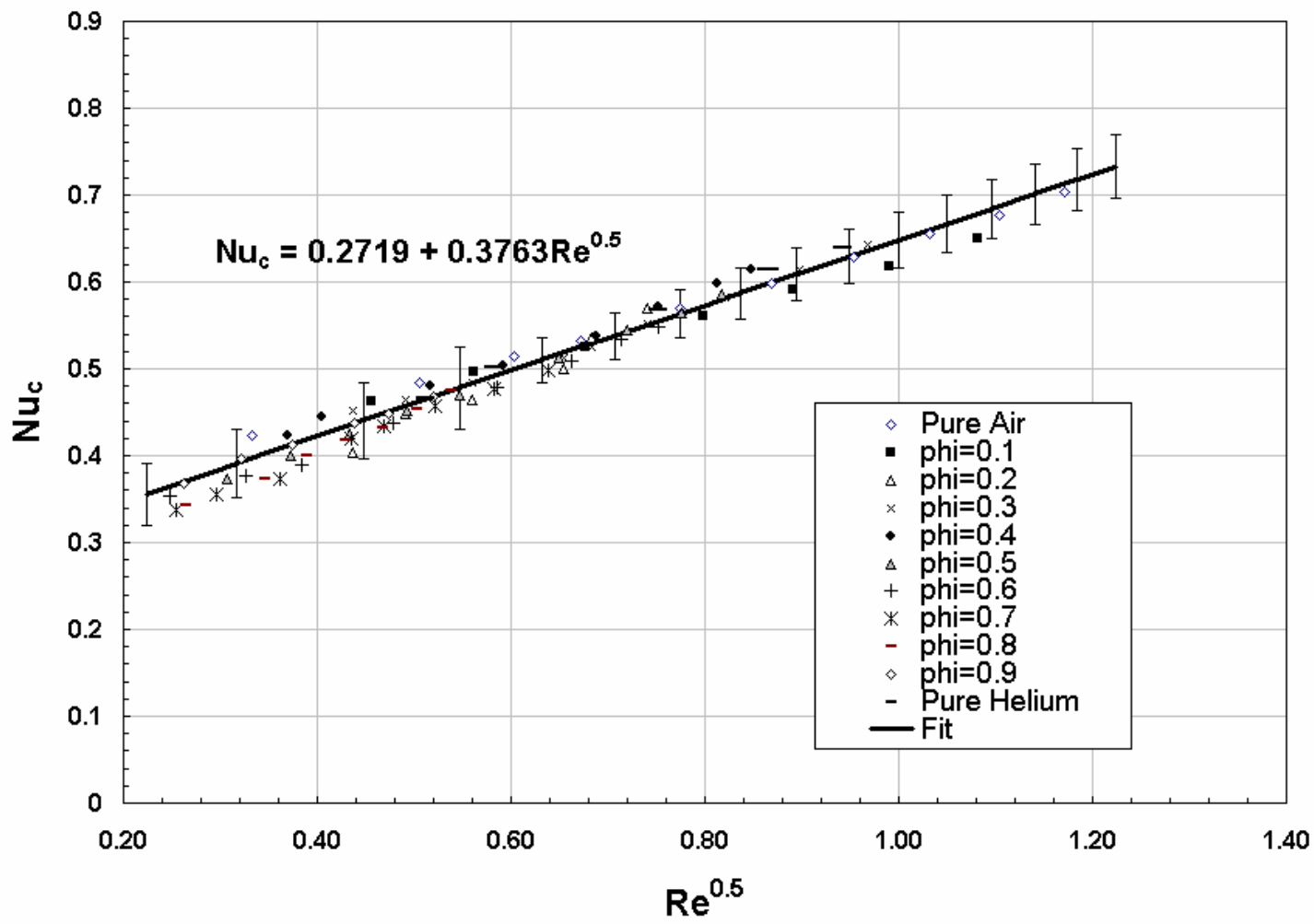

Figure 5.4 Heat transfer correlation in a binary gas mixture of air and helium. 


\section{EXPERIMENTAL RESULTS *}

\subsection{Preliminaries}

The following sections contain a discussion of the velocity and density data obtained (statistical only) using the Image and Hot-wire diagnostics techniques described in Sections 3 and 4. A wide range of Atwood number experimental runs $(0.035-0.25)$ were performed. Details about the various measurements can be found in Table 6.1. We define a dimensionless time, $\tau$, similar to the one defined by Dalziel et al. 1999; Ramaprabhu \& Andrews 2004; Snider and Andrews 1994. The downstream distance $x$ was converted to time $t$, using the Taylor hypothesis, $t=x / U$ (Taylor 1938 ).

$$
\tau=t\left(\frac{A_{t} g}{H}\right)^{1 / 2}=\frac{x}{U_{m}}\left(\frac{A_{t} g}{H}\right)^{1 / 2}
$$

The definition of $\tau$ incorporates the self-similar nature of the flow (here, $g$ is the acceleration due to gravity, $x$ is the downstream distance, $U_{m}$ is the mean convective velocity, and $H$ the depth of the channel). Hot-wire data was taken at various downstream locations from the splitter plate. The data presented here describes the more fully developed R-T mix. Various velocity, density and density-velocity statistics were measured using the hot-wire diagnostics. An extensive error/uncertainty analysis for the parameters measured was provided in Appendix B.

\footnotetext{
${ }^{*}$ Parts of this section including Figures $6.1,6.2,6.3,6.5,6.6$ and 6.9 have been reprinted with permission from Banerjee A, Andrews MJ. 2006. Statistically steady measurements of Rayleigh-Taylor mixing in a gas channel. Phys. Fluids 18:1-13
} 
TABLE 6.1 List of imaging and hot-wire experiments (Note: y-location of 0 corresponds to the centerline of the mix).

\begin{tabular}{|c|c|c|c|}
\hline Diagnostic & $\begin{array}{l}\text { x-location } \\
\text { (cm) }\end{array}$ & $\begin{array}{l}\text { Atwood } \\
\text { Number }\end{array}$ & $\begin{array}{l}\text { y-location } \\
\text { (cm) }\end{array}$ \\
\hline Imaging & $5-185$ & $\begin{array}{c}0.035 \text { and } \\
0.25\end{array}$ & - 45 to 45 \\
\hline $\begin{array}{l}\text { Multi-position } \\
\text { method }\end{array}$ & $\begin{array}{c}100,175 \text { and } \\
195\end{array}$ & 0.035 & 0 \\
\hline \multirow{2}{*}{$\begin{array}{c}\text { Multi-position } \\
\text { Multi- } \\
\text { Overheat } \\
\text { Method }\end{array}$} & $\begin{array}{c}50,75,100 \\
150,195\end{array}$ & \multirow[t]{2}{*}{0.04} & 0 \\
\hline & 175 & & $\begin{array}{c}-36 \text { to } 36 \mathrm{~cm} \\
\text { (in } 9 \mathrm{~cm} \\
\text { steps) }\end{array}$ \\
\hline
\end{tabular}




\subsection{Measurements with Visualization Analysis}

\subsubsection{Mixture Fraction Measurement}

Figures $6.1 \mathrm{a}-\mathrm{b}$ show a photograph of the buoyancy driven mixing layer with green smoke introduced in the helium-air mixture. Inspection of the figure reveals that large coherent structures in the light (air) and dark (air-helium-smoke) regions co-exist with finer scales of turbulent mixing. The figure also shows that small structures initially form (on the far right) and develop coherently to larger structures downstream (far left). The minimum intensity (dark) corresponds to an air-helium mixture (dyed with green smoke) fraction of zero and the maximum intensity corresponds to a pure air with a mixture fraction of 1. Measured mixture fractions were based on the linear relationship between the dye concentration and pixel intensity as described earlier. In particular, to calculate the fluid mixture fraction, peaks on the corrected image histogram were determined. These peaks represent the low $\left(\overline{I_{\min }}\right)$ and the high $\left(\overline{I_{\max }}\right)$ intensity for the test section and were ensemble averages. Once the minimum and maximum intensities corresponding were established, the fluid mixture fraction was determined from the linear relationship of concentration to intensity as:

$$
\overline{f_{V}}(x, y)=\frac{I(x, y)-\overline{I_{\min }}}{\overline{I_{\max }}-\overline{I_{\min }}}
$$

Figure 6.2 shows a plot of the $5 \%, 20 \%, 50 \%, 80 \%$ and $95 \%$ fraction contours superimposed on the average image ( $N=400$ images) taken during the Atwood number 0.035 run. It can be seen that the centerline ( $50 \%$ contour profile) was horizontal across 


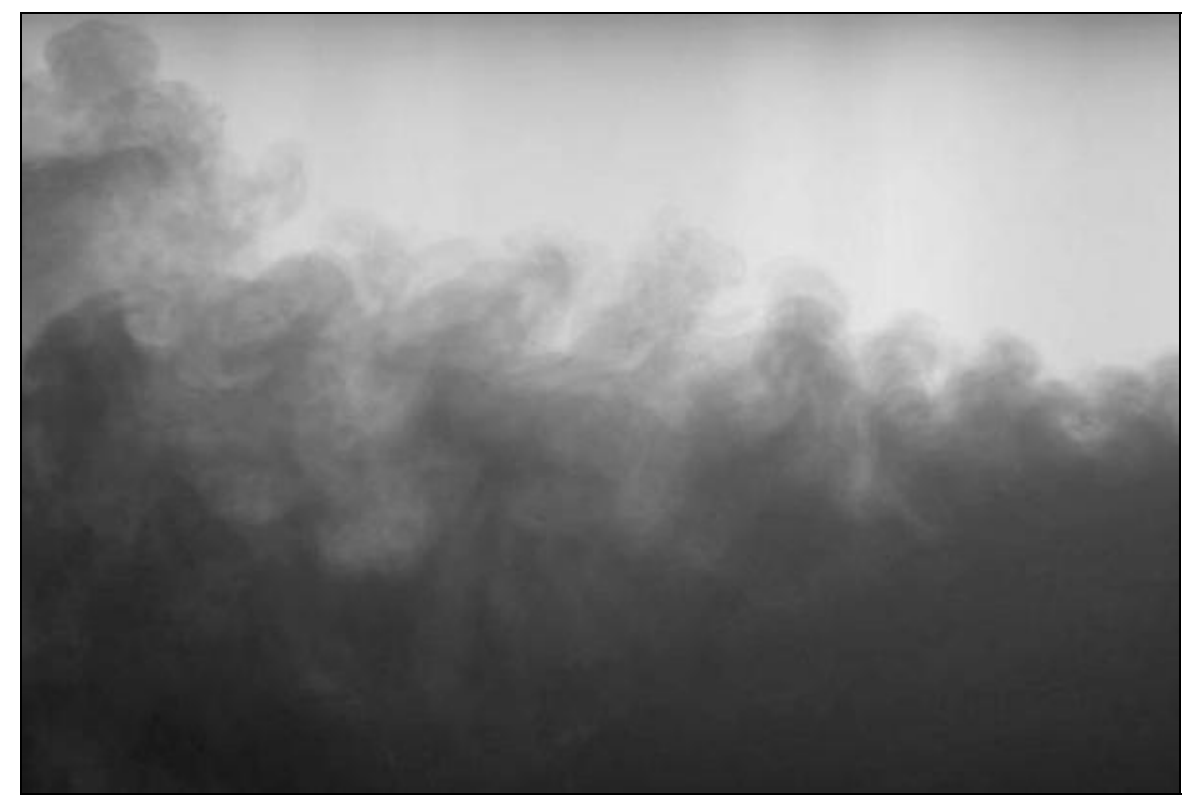

(a)

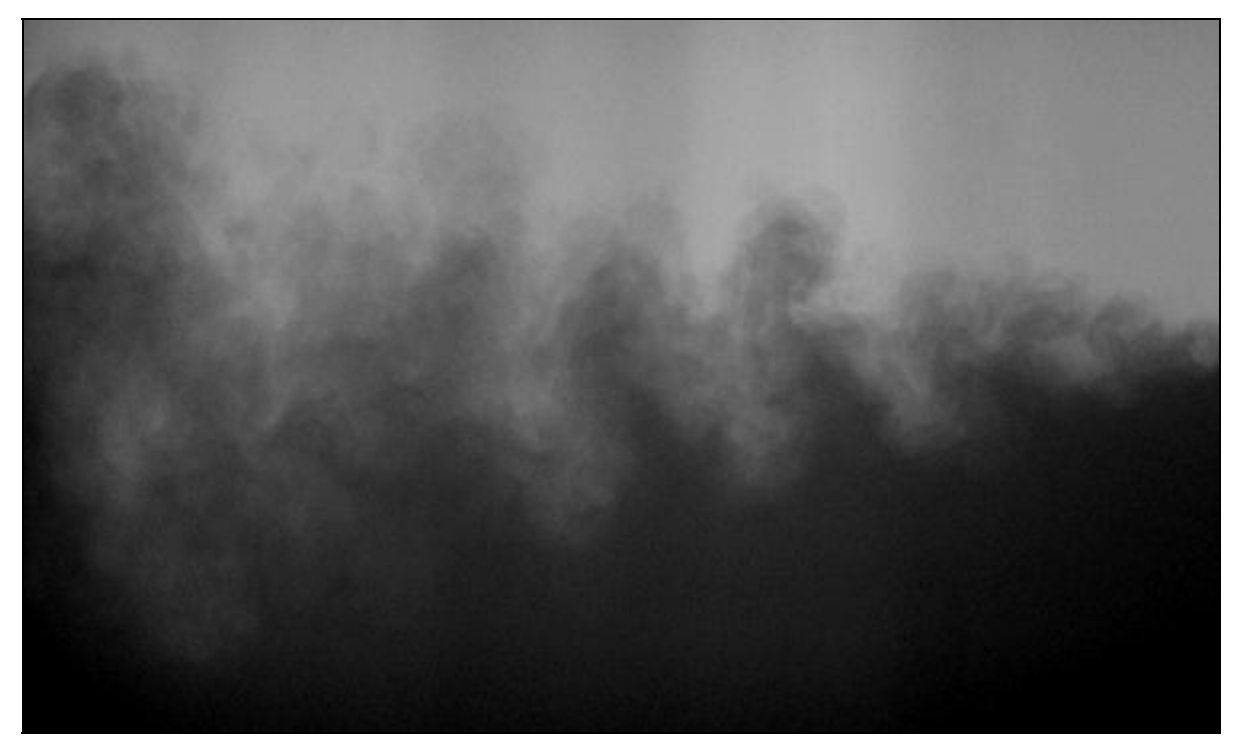

(b)

Figure 6.1 Close-up view of the three dimensional plumes across the channel for (a) $A_{\mathrm{t}} \#$ $0.04\left(U_{\mathrm{m}}=50 \mathrm{~cm} / \mathrm{s}\right)$ and $(\mathrm{b}) A_{\mathrm{t}} \# 0.097\left(U_{\mathrm{m}}=85 \mathrm{~cm} / \mathrm{s}\right)$. 


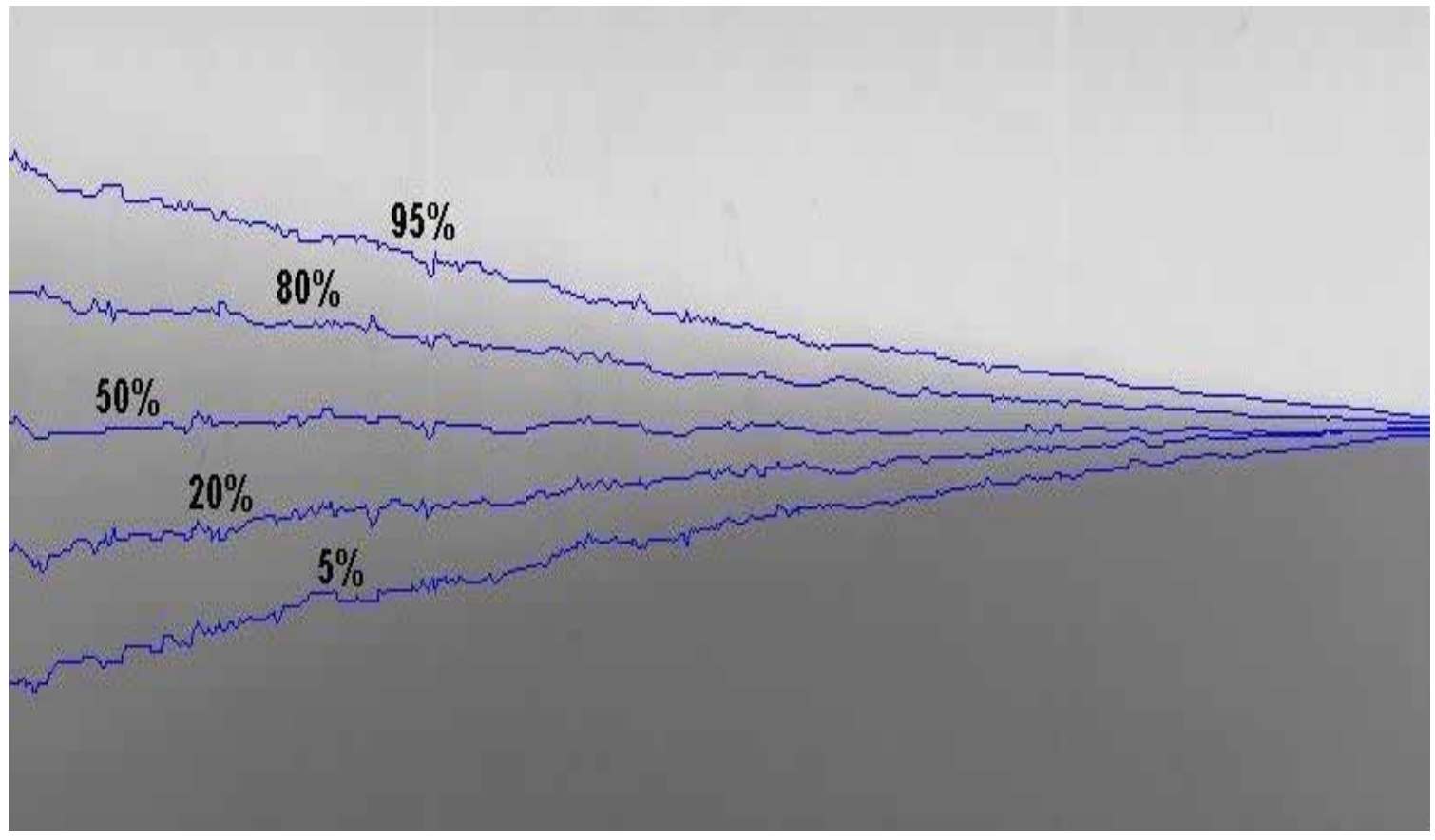

Figure 6.2 Contour levels $(5 \%, 20 \%, 50 \%, 80 \%$ and $95 \%)$ plotted on average image ( $N$ $=400)$ for an experimental run at $A_{\mathrm{t}} \# 0.035\left(U_{m}=0.6 \mathrm{~m} / \mathrm{s}\right)$. 
the length of the channel. The $5 \%$ and $95 \%$ contours show the spread of the mixing layer down the length of the channel. Figure 6.3 shows the measured fluid mixture fraction variation at three axial locations of $0.75 \mathrm{~m}, 1.4 \mathrm{~m}$ and $1.75 \mathrm{~m}$ (at three times) taken during an experimental run at Atwood number 0.035. The linear variation of mixture fraction suggests that the air and helium-air mixture were near linearly distributed across the mixing layer in the ensemble average of the turbulent buoyancy driven R-T mixing process. The results compare well with earlier experiments (Andrews, 1986 ; Dimonte, 1996 ; Snider and Andrews, 1994; Youngs, 1984) and also with numerical models (Dimotakis 2000; Youngs 1984). Figure 6.4 shows the mixture fraction profiles during an Atwood number run of 0.25 . Measured fluid mixture fraction variation at two axial locations of $0.5 \mathrm{~m}$ and $1.50 \mathrm{~m}$ are shown. It can be seen that the mix was slightly asymmetric with the heavier fluid penetrating more (spike) into the lighter stream.

\subsubsection{Mix Width Measurement}

There are a number of methods to measure the mixing width using a passive scalar. Following Andrews and Spalding(1990), the mix width can be measured as:

$$
2 h=6 \int_{0}^{H} \overline{f_{v}}\left(1-\overline{f_{v}}\right) d y
$$

where, the integral can be evaluated from mixture fraction data measured over the width of the channel as in Equation 6.3 the mixing layer. However, this method does poorly at narrow mixture widths (close to the splitter plate) and was sensitive to noise (Snider \& Andrews 1994). A more accurate method to measure the mixing width was to measure the distance between the 0.05 and 0.95 fraction profiles (Andrews \& Spalding 1990). 


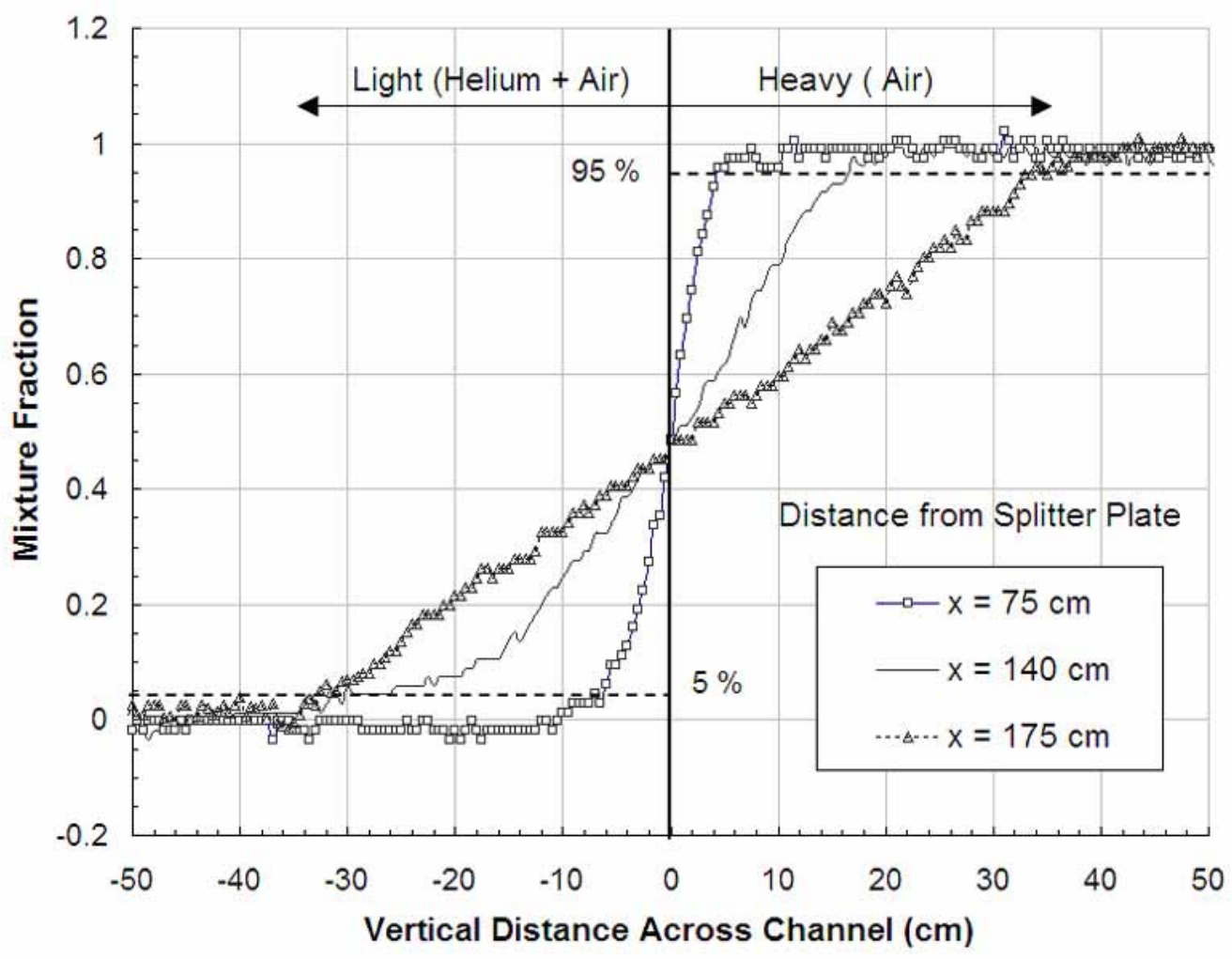

Figure 6.3 Mixture fraction distributions across the mixing layer for $A_{\mathrm{t}} \# 0.035$. (Note: For errors in mixture fraction measurements, see Appendix B.4) 


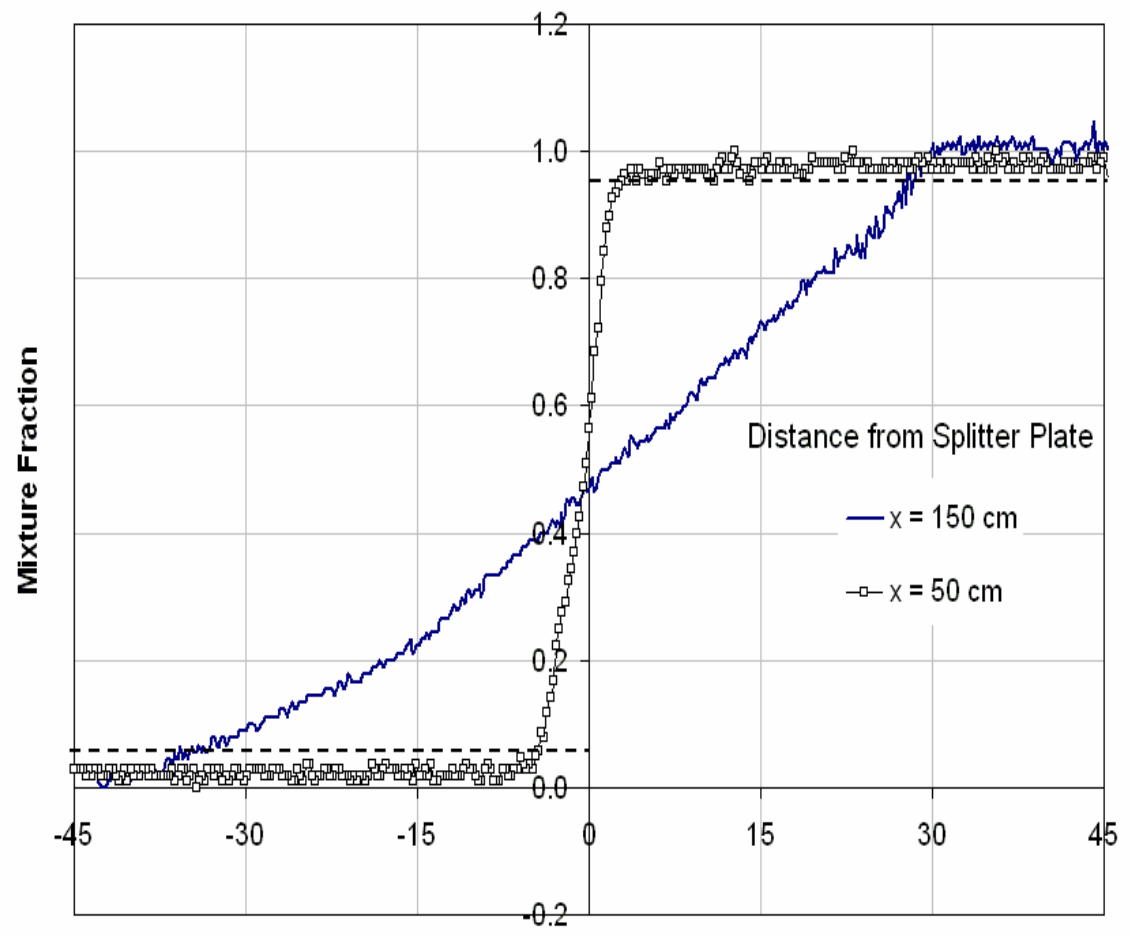

Vertical Distance Across Channel (cm)

Figure 6.4 Mixture fraction distribution across the mixing layer for $A_{\mathrm{t}} \#$ 0.259. (Note: For errors in mixture fraction measurements, see Appendix B.4) 


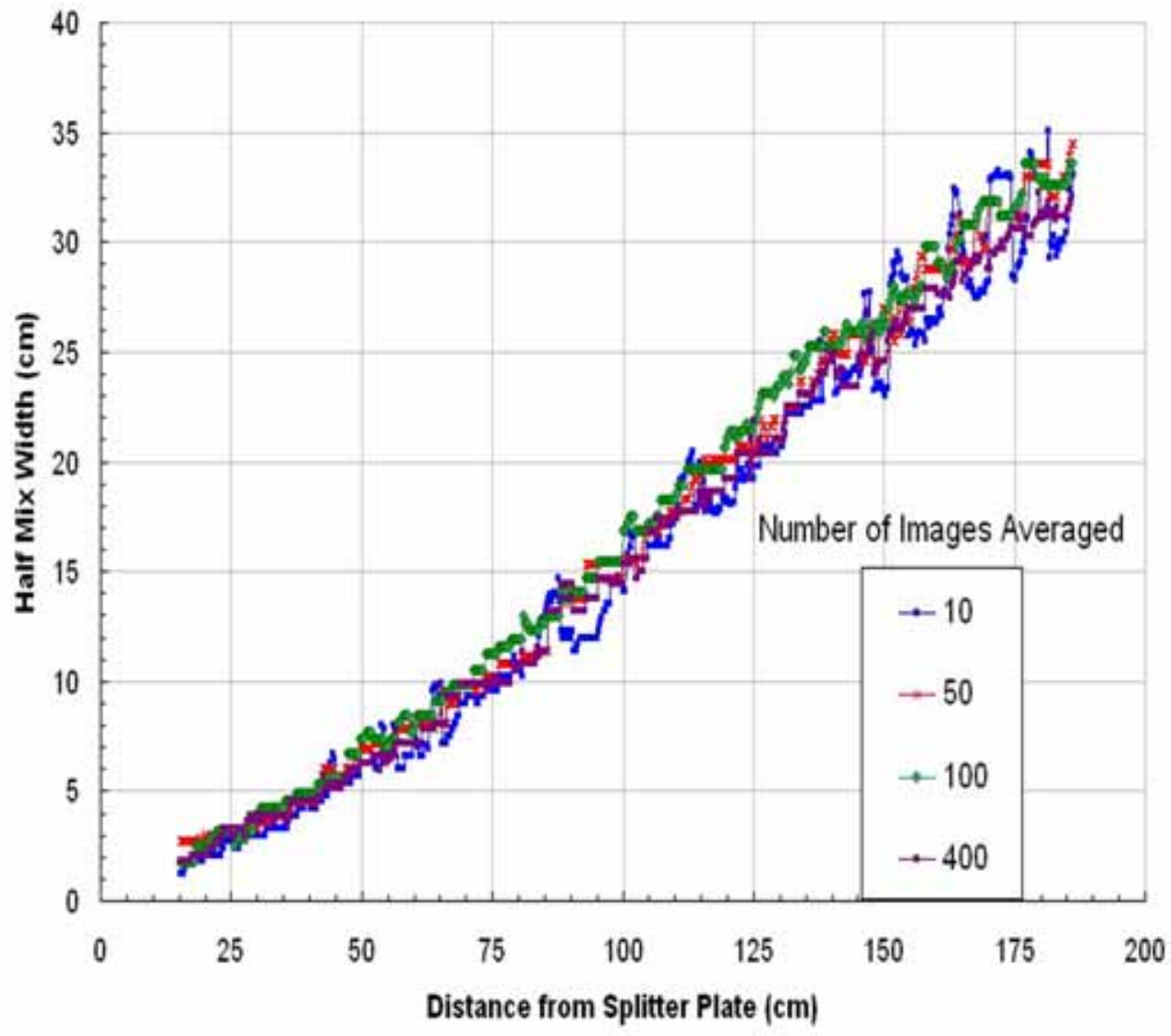

Figure 6.5 Effect of number of images in average on the mixing width $\left(A_{\mathrm{t}} \# 0.035\right)$. 
During an experiment, the velocities were set to let the mix reach the top and bottom walls of the channel close to the exit plenum, thus keeping the mix spread angle below $15^{\circ}$ and thus parabolic (Snider \& Andrews 1994). The mixture width was measured from ensemble averaged images. The sensitivity to the number of images used on the average was evaluated by comparing mixture widths from 10, 50, 100 and 400 images. Figure 6.5 shows that the average of 400 images compares well with that from the data of 100 images. It was found that an ensemble average of 100 to 400 images produced a consistent mixture width measurement.

\subsubsection{Test of Convergence}

Uncertainties in density profiles occur due to non uniform smoke intensity as can be seen near the edge of the mixing layer. To verify that these errors were indeed measurement errors and not as a result of lack of statistical convergence, a detailed statistical convergence test was performed (Ramaprabhu \& Andrews 2003) . Assuming that the errors in the mixture fraction (density) measurements were purely random, it was observed that the standard deviation of the density at the edge of the mix falls on a $\chi^{2}$ distribution (Ramaprabhu \& Andrews 2003). If $n$ denotes the number of degrees of freedom in each sample, i.e. $n=N-1$, then the relationship between the sample mean $\sigma^{2}$ and the population mean $s_{0}^{2}$ can be written as:

$$
s_{0}^{2}=\frac{\sigma^{2}}{n} \cdot \chi_{n}^{2}
$$


where, $\chi_{n}^{2}$ was the $\chi^{2}$. function based on $n$ degrees of freedom. An interrogation window far downstream and close to the edge of the mix was considered. Thus for a given sample size $N$ and a $95 \%$ confidence level, the $\chi^{2}$. distribution was evaluated which gave an estimate of the error in determining density as shown in Figure 6.6. The convergence of more than 150 images was also confirmed from the plot of the mix width (Figure 6.5). Thus the errors associated with the experimental data from the images were a result of the inherent randomness of the measurement process. The size of the interrogation window also affects the error. An argument similar to the one used in continuum hypothesis was used. A small window would make the local spatial average statistically unreliable as it was based on too few data points. On the other hand, a large window would cause significant flow structures in the field to alter the averages. Thus a plot of intensity versus interrogation window size was plotted at the edge of the mix. It can be seen in Figure $6 b$ that for a window size of 10 to 18 , the computed averages were not dependent on the size of the interrogation window as it falls in the plateau region in the plot. Thus an interrogation window size of $16 \times 16$ was selected for testing of convergence.

The results were compared with a similar analysis performed with PIV images (Ramaprabhu \& Andrews 2004). The current analysis was performed by digital image analysis of the flow section where each image contains information from a number of mix planes along the depth of the channel (image). Thus each image contains information from a number of large structures, both along the direction of flow and also 


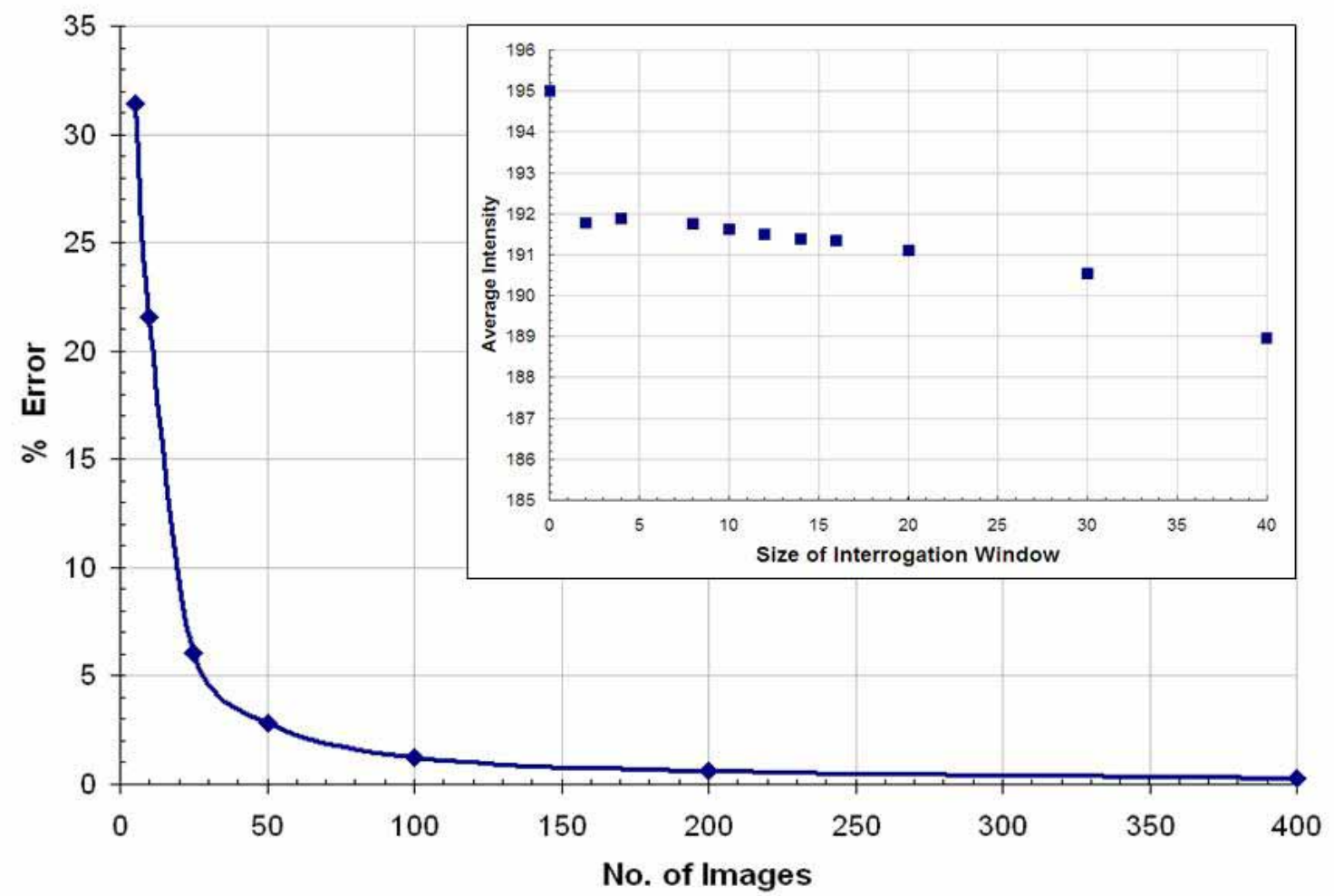

Figure 6.6 Percentage (\%) root mean square error of density as a function of number of images $(16 \times 16$ interrogation window) [Inset shows average intensity within an interrogation window plotted as a function of the window size (in pixels)]. 
across the depth of the channel. In the current study, the images were captured at a rate of 80 images/ minute over a 5 minute period, so that a large number of large structures were captured in the 400 images used for convergence. In comparison, the PIV image analysis (Ramaprabhu \& Andrews 2004) was inherently two dimensional and the average contained less information. More importantly, the PIV images were captured at a frequency of $30 \mathrm{~Hz}$ and a set of 2100 images over a run time of $\sim 70$ seconds. Since the flow speed was significantly less $(\sim 5 \mathrm{~cm} / \mathrm{s})$, the number of large structures required to obtain statistical convergence was obtained by averaging over 2000 images. Although the statistical convergence in the present study was attained with a lesser number of images, it was concluded that the statistical convergence was a direct consequence of the number of different large 3D structures that were averaged over the duration of the run. So the results were consistent with earlier findings (Ramaprabhu \& Andrews 2004).

\subsubsection{Growth Constant $(\alpha)$ Measurement}

In far-field (i.e. at late time), the ensemble averaged half mixing width $h$ was expected to grow as:

$$
h=\alpha_{b} A_{t} g t^{2}=\alpha_{b} A_{t} g\left(\frac{x}{U_{m}}\right)^{2}
$$

where $\alpha_{b}$. was expected to be constant at the low Atwood studies performed here. From Figure 6.4 , it can be seen that although the data follows a near non-linear trend, the mixing half width does not go to zero at the start of the test section (end of splitter plate). This implies a virtual origin at the start of the splitter plate that accounts for the effect of the splitter plate and the non-similarity of the initial mixing layer. Snider (1994) used an 


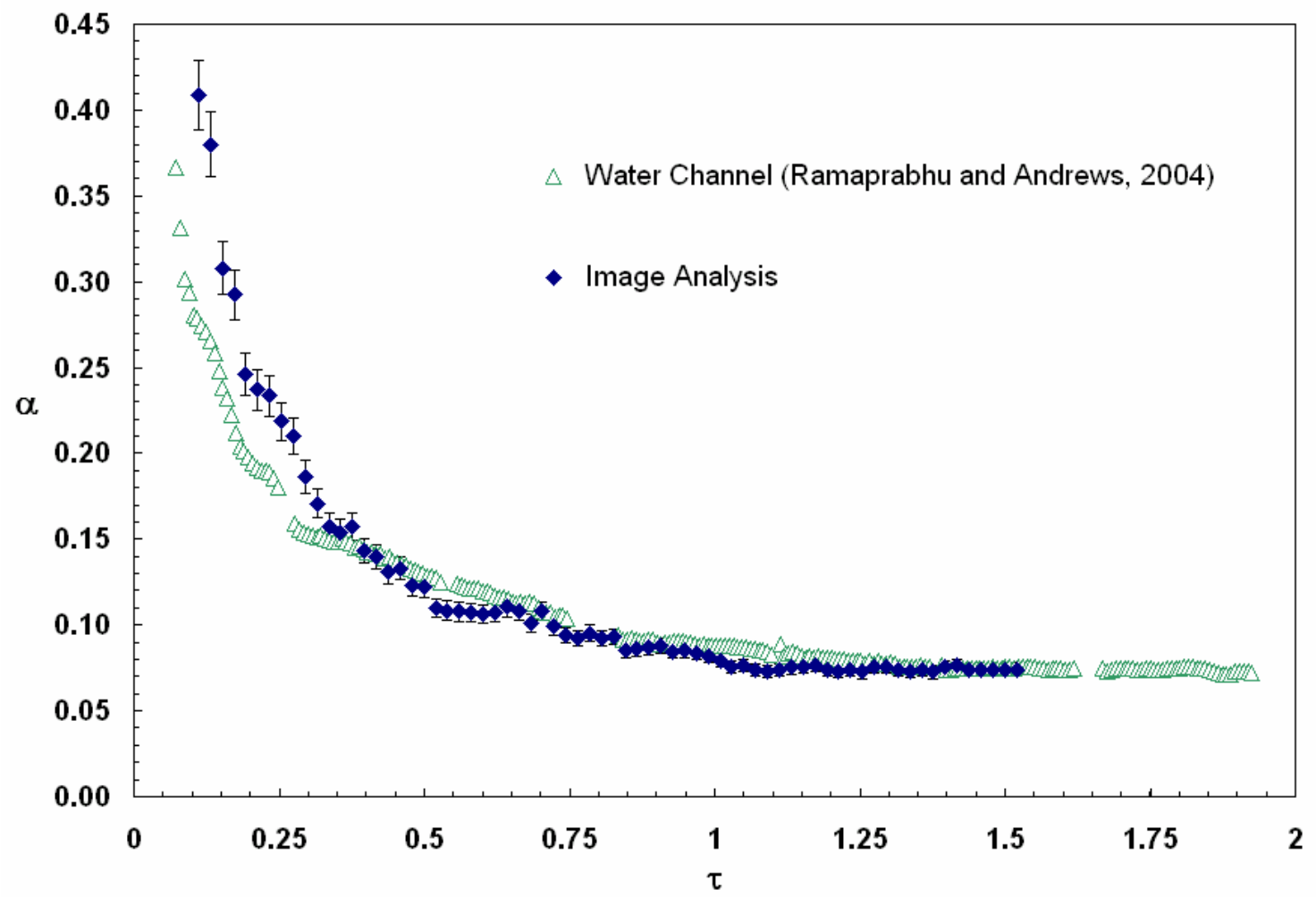

Figure 6.7 Comparison of growth constant $\left(\alpha_{b}\right)$ measured with image diagnostics (gas channel) and PIV (water channel). 
absolute deviation algorithm which defines a virtual origin and fits a linear curve to the mixing-half width to evaluate the growth constant. However, this technique was subject to the accuracy of the fitting technique, but was accurate for getting a late-time saturated estimate of $\alpha_{b}$. In the current study, a program was written that determined $\alpha$ based on a "moving window" calculation. This technique used the half-mix width data as discussed earlier in section $\mathrm{C}$. A window with 10 pixels $(2.5 \mathrm{~cm})$ contained 10 mixing half widths at each pixel location. The window was used to calculate a slope $(d h / d x)$ for the mixing region by determining a $2^{\text {nd }}$ order polynomial best fit line through the data using a method of least squares. The slope of the best fit line was used to calculate $\alpha$ within the "window". The window stepped along the downstream direction 1 pixel at a time and calculated $\alpha$ at each location. The slope $(d h / d x)$ was used here as a measure of $\alpha$ to better compare with the value of growth constant measured from hot-wire measurements using the vertical velocity fluctuations $v^{\prime}$ at the center-line (see Equation 6.5). Figure 6.7 shows a plot of $\alpha$ as a function of non-dimensional time $\tau\left[=x / U_{m} \cdot\left(A_{t} g / H\right)^{0.5}\right]$, where $H$ was the height of the channel $(1.2 \mathrm{~m})$. Inspection of Figure 6.7 reveals that $\alpha$ asymptotes to a value of $0.065-0.07$ suggesting that the flow reaches self-similarity in this experiment $\left(A_{\mathrm{t}} \# 0.035\right)$. Similar observations were recorded in earlier small Atwood $\left(A_{\mathrm{t}} \sim 10^{-3}\right)$ studies (Ramaprabhu \& Andrews 2004). The value of $\alpha$ measured at the various Atwood number runs in the Gas Channel were compared with the LEM (Linear Electric Motor) experiments of Dimonte (1996, 1999). As seen in Figure 6.7, our measurements agree reasonably. 


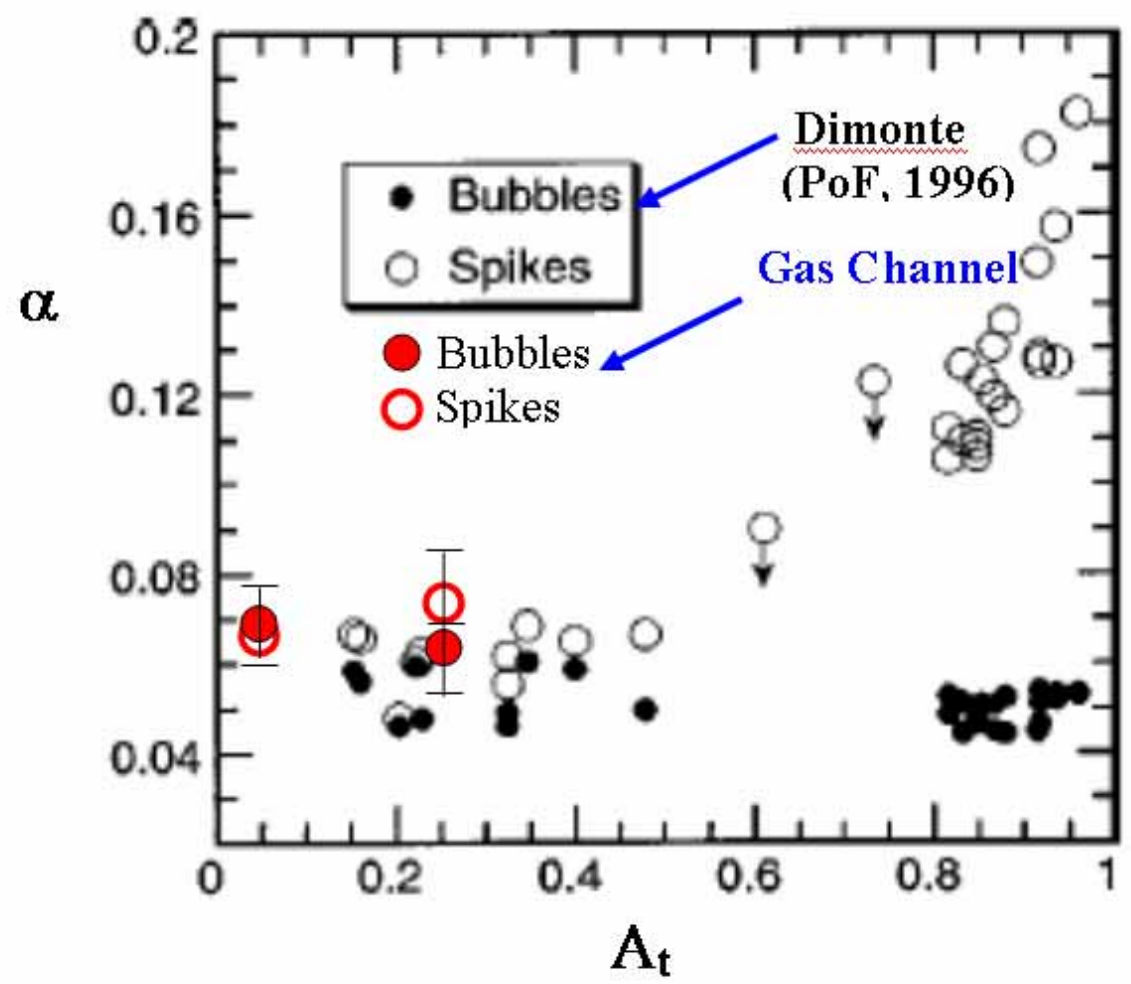

Figure 6.8 Comparison of growth constant $(\alpha)$ obtained by image analysis (at gas channel) with LEM (Linear Electric Motor) experimental measurement of Dimonte \& Schneider (1996) 


\subsection{Hot-Wire Measurements}

\subsubsection{Measurements with Multi-position SN-Wire Technique}

The density gradient needed to create a RT Instability was obtained by using a heavy fluid (air) over a light one (air-helium mixture). This was different from the low Atwood water channel experiments where the instability was created using cold water (heavy) over hot water (light). However, the addition of helium to the mix caused a small temperature gradient $\left(\sim 2^{\circ} \mathrm{C}\right)$ along the vertical $(y)$ direction (i.e. from the air to the air/helium mixture). As already mentioned before, we took advantage of this small temperature gradient and used a cold wire probe couple to a CCA unit to check for the centerline of the mix. Initially, the cold-wire was placed close to the centerline of the mixing layer by visual inspection and the data was logged. The centerline offset factor $\phi$ was computed after the logging process as:

$$
\phi=\left[1+\frac{C_{p}^{\text {bottom }}}{C_{p}^{\text {top }}} \frac{\rho_{\text {bottom }}}{\rho_{\text {top }}}\left(\frac{T_{\text {bottom }}-T_{i}}{T_{i}-T_{\text {top }}}\right)\right]^{-1}
$$

where, $C_{p}^{\text {bottom }}$ and $T_{\text {bottom }}$ were the specific heat and temperature of the helium-air mixture (flow at the bottom section of the channel); $C_{p}^{\text {top }}$ and $T_{\text {top }}$ were the specific heat and temperature of air (flow at the top section of the channel); and $T_{i}$ was the temperature of the mix. If $\phi \neq 0.5$, the position of the cold-wire was readjusted and the data logging process repeated. The cold-wire was adjusted until $\phi$ approaches a value of 0.5 with an accuracy of $5 \%$. 
The hot-wire was placed at the center-line location at various downstream positions from the splitter plate $(x=1.0 \mathrm{~m}, 1.75 \mathrm{~m}$ and $1.95 \mathrm{~m})$. The centerline r.m.s. values of the vertical, $v^{\prime}$, horizontal, $u$ ', and cross wise, $w^{\prime}$ velocities were determined using the multiposition single wire technique discussed in Section 4.3.1 at $\tau$ locations of $0.8126,1.422$ and 1.5845 (for $x=1.0 \mathrm{~m}, 1.75 \mathrm{~m}$ and $1.95 \mathrm{~m}$ ) from the splitter plate. The results were tabulated in Table 6.2. Ramaprabhu and Andrews (2004) found that the vertical velocity fluctuation $\left(v^{\prime}\right)$ at the centerline can be related to the centerline of the mix width by:

$$
v^{\prime}=\frac{d h}{d t}=2 \alpha A_{t} g t=2 \alpha A_{t} g \frac{x}{U_{m}}
$$

Thus, by using Equation 6.7 and the centerline value of v', we imply that the expansion of the mix was driven by velocity fluctuations that occur across the whole mix and not just the edge. This characterization was also supported by observations of the mixing layer shown in Figure 6.1, where large-scale structures span the mix and dominate the velocity fluctuations. Statistics measured in this region remain practically constant across the mix. Thus it was convenient to determine the growth constant $\alpha$ by computing the ratio, $v^{\prime} /\left\{2 A_{t} g\left(x / U_{m}\right)\right\}$. The measured ratio at the 3 downstream locations was plotted as a function of non-dimensional time $\tau$ in Figure 6.9. It was seen that the results agree with the value of $\alpha$ as found from image analysis by using a moving window technique. Since the Atwood number of 0.035 used for the study was low, it was expected that the dynamics of the R-T mix should be similar to that of the small Atwood 
TABLE 6.2 Velocity fluctuations $(\mathrm{m} / \mathrm{s})$ measured at $A_{\mathrm{t}} \# 0.035\left(U_{\mathrm{m}}=0.6 \mathrm{~m} / \mathrm{s}\right)$

\begin{tabular}{|c|c|c|c||}
\hline Distance from & 1.0 & 1.75 & 1.95 \\
splitter plate: $x(\mathrm{~m})$ & & & \\
\hline$u^{\prime}$ & $0.0879 \pm 0.002$ & $0.0724 \pm 0.001$ & $0.0837 \pm 0.0015$ \\
\hline$v^{\prime}$ & $0.1051 \pm 0.001$ & $0.1286 \pm 0.0012$ & $0.1440 \pm 0.0014$ \\
\hline$w^{\prime}$ & $0.0729 \pm 0.002$ & $0.0709 \pm 0.001$ & $0.0753 \pm 0.0015$ \\
\hline$u^{\prime} / U_{m}$ & 0.1353 & 0.1114 & 0.1287 \\
\hline$v^{\prime} / U_{m}$ & 0.1617 & 0.1978 & 0.2216 \\
\hline$v^{\prime} / u^{\prime}$ & $1.1951 \pm 0.029$ & $1.7747 \pm 0.0369$ & $1.7218 \pm 0.0339$ \\
\hline$\alpha$ & $0.1004 \pm 0.02$ & $0.0702 \pm 0.012$ & $0.0706 \pm 0.011$ \\
\hline$\tau=\frac{x}{U_{m}}\left(\frac{A_{t} g}{H}\right)^{0.5}$ & 0.8126 & 1.4220 & 1.5845 \\
\hline & & & \\
\hline & & & \\
\hline
\end{tabular}




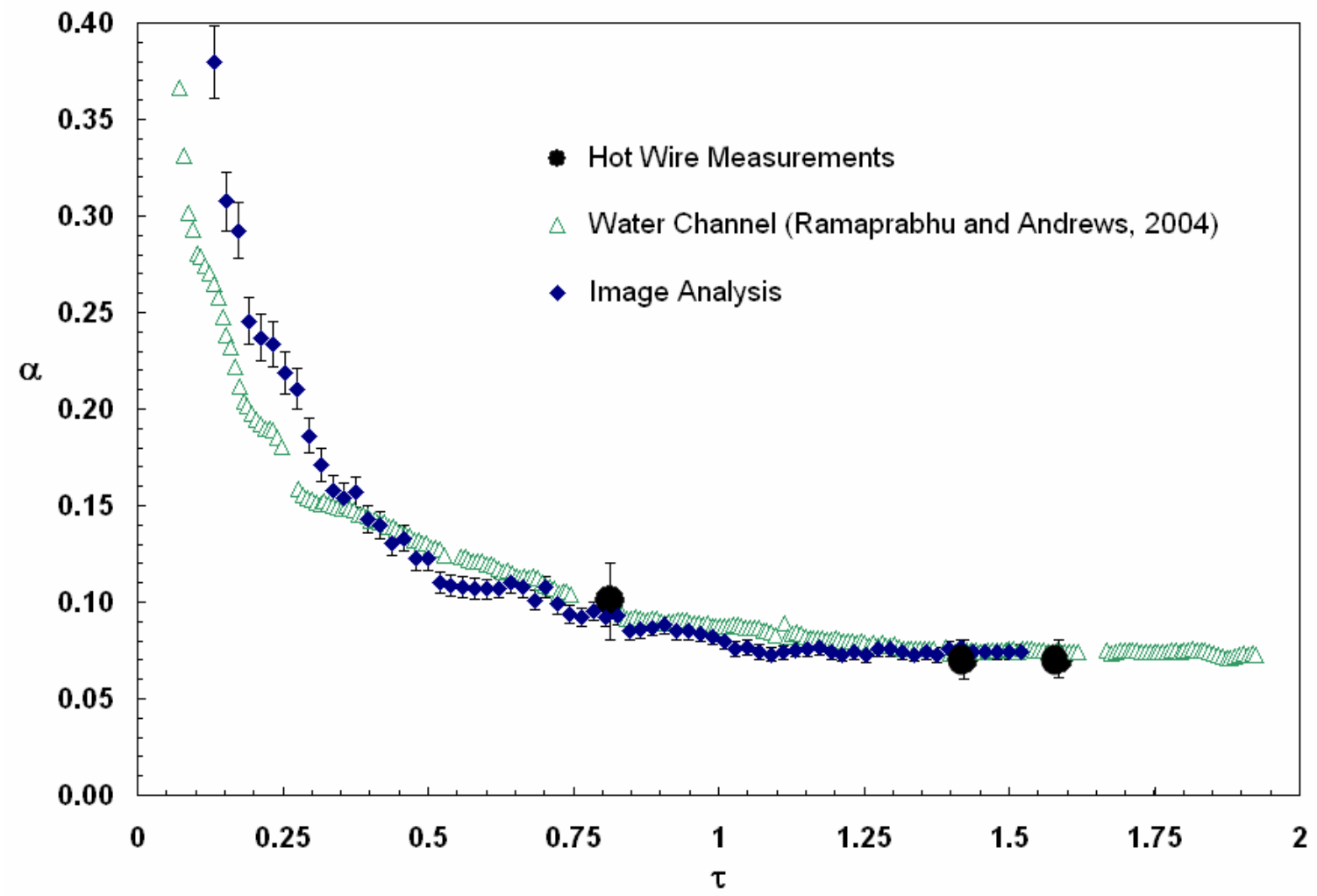

Figure 6.9 $\alpha_{\mathrm{b}}$ at the centerline plotted as a function of $\tau$. 
number runs in the water channel facility. Thus the growth constant obtained by PIV measurements for a small Atwood run in the water channel was also plotted in Figure 6.9. All three sets of measurements of $\alpha$ agree reasonably well, and give a late time value of $0.065-0.07$. This consistency across measurement techniques (visualization, PIV and hot-wire anemometry) and experiments (hot/cold water low Atwood facility, air/heliumair high Atwood facility) demonstrated that the new facility provides consistent statistics for the present experiment. The saturation of $\alpha$ at late time to a constant value of 0.07 was consistent with the measurements made at the small Atwood water channel facility (Ramaprabhu \& Andrews 2004). For low values of Atwood numbers used in the present study, the value of $\alpha_{b}$ is expected to be same for both bubbles and spikes (as the structures were symmetric). However for high Atwood numbers ( $\geq 0.5)$, the value of $\alpha$ would be different as the flow will be no longer symmetric but characterized by rising bubbles and falling spikes (Dimonte 1999; Dimonte \& Schneider 1996). So for higher Atwood number ( $>0.1)$, Equation 6.7 would not give the same $\alpha$ as that based on mix width measurements. However, it provides a well defined method for calculating an $\alpha$ based on centerline velocity fluctuations that may be more convenient for validation purposes (Banerjee \& Andrews 2006).

\subsubsection{Measurements with Multi-position Multi-Overheat Technique}

The multi-position multi-overheat technique takes into account the density variations in the flow and was used to measure velocity correlations $\left(\overline{u^{\prime 2}}, \overline{v^{\prime 2}}, \overline{u^{\prime} v^{\prime}}\right)$; velocitydensity correlations $\left(\overline{\rho^{\prime} u^{\prime}}, \overline{\rho^{\prime} v^{\prime}}\right)$; as well as molecular mix parameters $\left(\theta, B_{0}\right.$ and $\left.B_{2}\right)$. 
Measurements were made at various downstream locations along the channel (see Table 6.1). Snider and Andrews (1994) has reported experiments which show that the side walls do not affect the central region of the mix in the experimental set-up. The range of density fluctuations and the extent of mixing in a Rayleigh-Taylor flow were characterized by the following parameters:

$$
\begin{aligned}
& f_{1}=\lim _{T \rightarrow \infty} \frac{1}{T} \int_{0}^{T} \frac{\rho-\rho_{2}}{\rho_{1}-\rho_{2}} d t=\lim _{T \rightarrow \infty} \frac{1}{T} \int_{0}^{T} \frac{\rho-\rho_{h}}{\rho_{c}-\rho_{h}} d t \\
& f_{2}=1-f_{1} \\
& B_{0}=\lim _{T \rightarrow \infty} \frac{1}{T} \int_{0}^{T}(\rho-\bar{\rho})^{2} d t / \Delta \rho^{2}=\rho^{\prime 2} / \Delta \rho^{2} \\
& B_{2}=f_{1} f_{2}=f_{1}\left(1-f_{1}\right) \\
& \theta=1-\frac{B_{0}}{B_{2}}
\end{aligned}
$$

where, $f_{i}$ is the fraction by volume of the $i^{\text {th }}$. fluid (with $f_{l \cdot}=f_{\text {heavy }}$, and $f_{2}=f_{\text {light }}$ ). $B_{0}$ is the intensity of turbulent density fluctuations and includes the effect of mixing due to molecular diffusion. $B_{2}$ is the corresponding conditioned measure that would result if the two fluids were immiscible and does not account for molecular mixing. Figure 6.10 plots $f_{1 .}$ (defined as $\frac{\bar{\rho}-\rho_{2}}{\rho_{1}-\rho_{2}}$, where $\bar{\rho}$ is the time-averaged density) across the mixing layer. As might be expected at the present low Atwood number run the mix is symmetric about the centerline. The total mix-width, $2 h$, was measured as the distance between the locations where the average density was $5 \%$ and $95 \%$ of the heavier fluid (air).

The parameter $\theta$ (Danckwerts 1952) quantifies the degree of mixing with $\theta=0$ implies no mixing, and $\theta=1$ corresponding to completely molecularly mixed fluids. As 


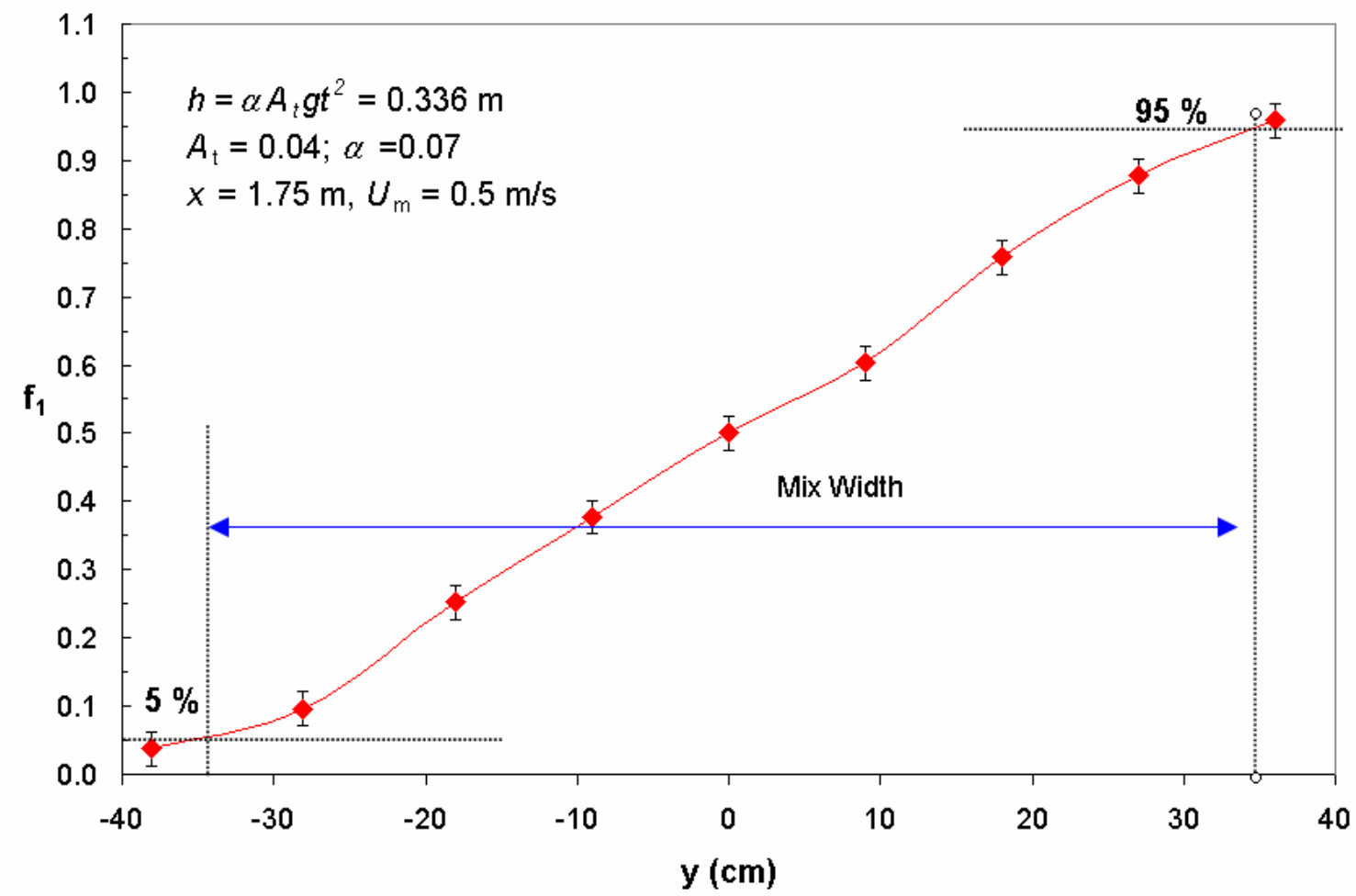

Figure 6.10 Density profiles measured with the multi-position multi-overheat hot-wire technique. 



Figure 6.11 Molecular mix parameters across the mix at $\tau=1.986\left(A_{\mathrm{t}} \# 0.04, x=1.75 \mathrm{~m}\right.$ from the splitter plate). The water channel measurements are by Wilson (2002). 
explained in more detail later, $B_{2}$ may also be interpreted as an intermittency factor based on the density of the fluids. The molecular mix $\theta$ affects the overall growth rate of the mix in the following way. Consider the definitions of $B_{0}$ and $B_{2}$ as the nondimensional turbulent density fluctuations in the presence and absence of molecular diffusion respectively. Then,

$$
B_{0}=\overline{\rho_{0}^{\prime 2}} / \Delta \rho^{2}, \quad B_{2}=f_{1} f_{2}=\overline{\rho_{2}^{\prime 2}} / \Delta \rho^{2}
$$

where the $\overline{\rho_{0}^{\prime 2}}$ and $\overline{\rho_{2}^{\prime 2}}$ refer to the turbulent density fluctuations for the miscible and 2fluid cases respectively, and $\Delta \rho=\rho_{1}-\rho_{2}$. The growth rate of the mix may be rewritten using the modified driving term $2 \sqrt{\overline{\rho_{0}^{\prime 2}}}$ (Ramaprabhu \& Andrews 2004)

$$
h=2 g t^{2} \alpha \sqrt{\overline{\rho_{0}^{\prime 2}}} /\left(\rho_{1}+\rho_{2}\right)
$$

This was a more natural choice for molecularly mixed fluids, since the density difference available to drive the flow $\Delta \rho=\rho_{1}-\rho_{2}$ was reduced in the presence of diffusion. For a 2-fluid case at the centerline where $f_{1 .}=f_{2 .}=0.5$, then

$$
\overline{\rho_{0}^{\prime 2}}=\overline{\rho_{2}^{\prime 2}}=f_{1} f_{2} \Delta \rho^{2}=\left(\rho_{1}-\rho_{2}\right)^{2} / 4
$$

In this case, Equation 6.10 reduces to Equation 1.1.

For miscible fluids, we have

$$
\frac{\overline{\rho_{0}^{\prime 2}}}{\overline{\rho_{2}^{\prime 2}}}=\frac{B_{0}}{B_{2}}=1-\theta
$$

Equation 6.11 thus reduces to

$$
h=\tilde{\alpha} A_{t} g t^{2}
$$




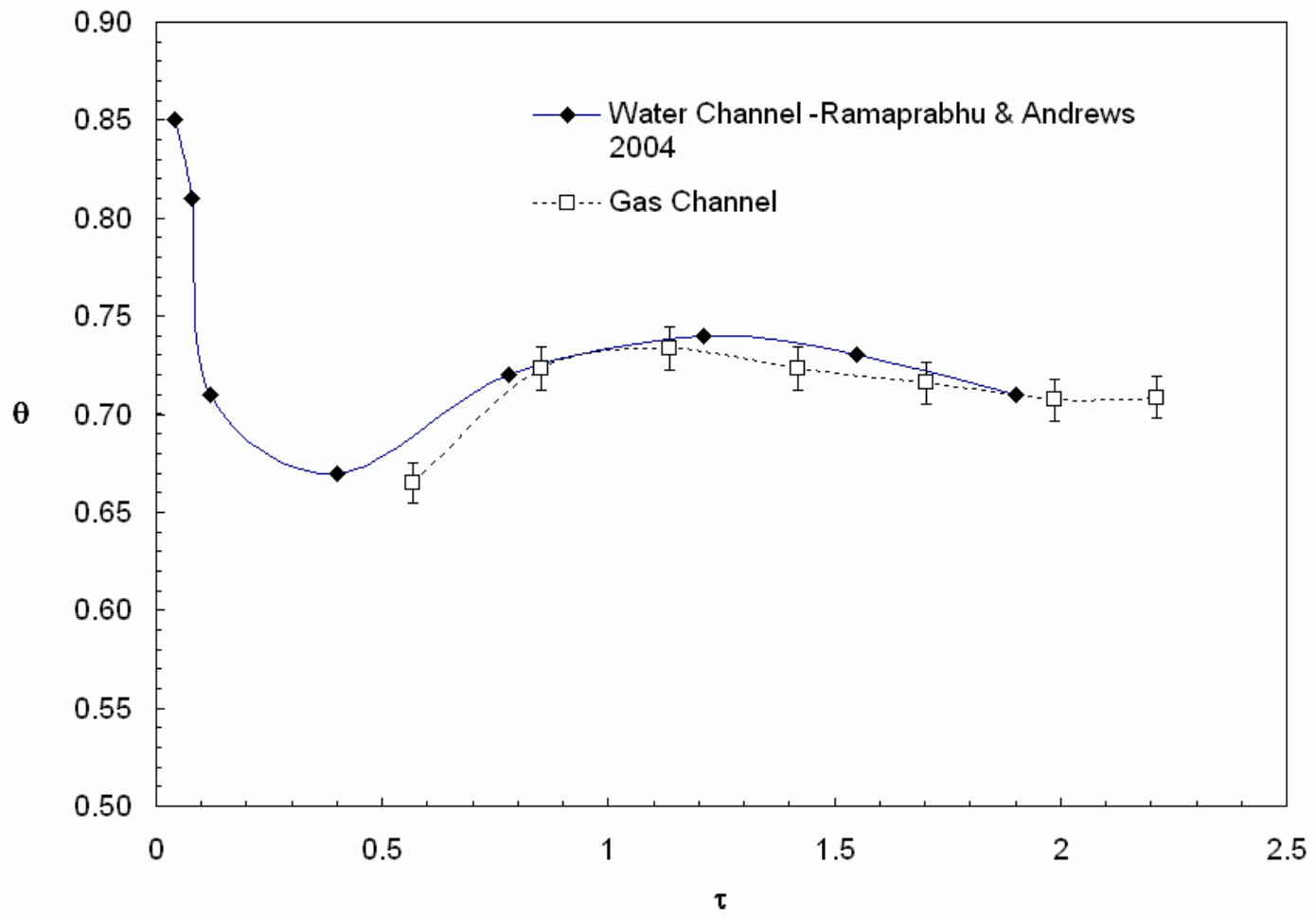

Figure 6.12 Time evolution of scalar turbulence intensity and mix parameters measured at the centerline $\left(A_{t} \# 0.04\right)$. Water channel measurements are by Ramaprabhu and Andrews (2004). 
where $\tilde{\alpha}=\alpha \sqrt{1-\theta}$. At the centerline of the mix, measurements of $\theta$ have been found to vary from 0.7 to 0.8 (Ramaprabhu and Andrews 2004), and remain reasonably constant across the mix (see Figure 6.11). Assuming an intermediate value of $\theta=0.72$, the presence of molecular diffusion serves to reduce the growth rate of the mix by a factor of 0.53. The front tracking calculations of Glimm et al. (2001) suggest a 2-fluid value for $\alpha$ of 0.07 , giving a net growth constant $\widetilde{\alpha}=0.53 \alpha=0.037$.

Figure 6.11 plots the molecular mix parameters across the mix. $\theta$ remains reasonably constant across the mix and this is consistent with earlier results (Ramaprabhu \& Andrews 2004; Wilson 2002). It is seen that towards the edge of the mix, where the probe sees more of one fluid than the other, the value of $\theta$ increases. The centerline timeevolution of the mix-parameters defined above and determined from the hot-wire measurements, were shown in Figure 6.12. Ramaprabhu and Andrews (2004) reported measurements of $\theta$ close to the splitter plate $(\tau \sim 0.034)$. The mixing layer was diffuse and, as a result, $\theta$ values were high in this region (and corresponding $B_{0}$. values were low). As the mix develops farther downstream, the fluctuation levels increase with the onset of the instability, resulting in a slight drop in the value of $\theta$. The measurements made at $x=50 \mathrm{~cm}(\tau \sim 0.567)$ correspond to this region. This effect was the result of stretching of the initial diffuse layer, followed by rolling up into mushroom-shaped structures. Eventually ( $\tau \geq 1.135$ ), turbulent diffusion serves to smooth out some of these fluctuations. As expected, the two-fluid parameter $B_{2}$ remains relatively constant at a value of 0.25 . This further confirms that these measurements were indeed at the 


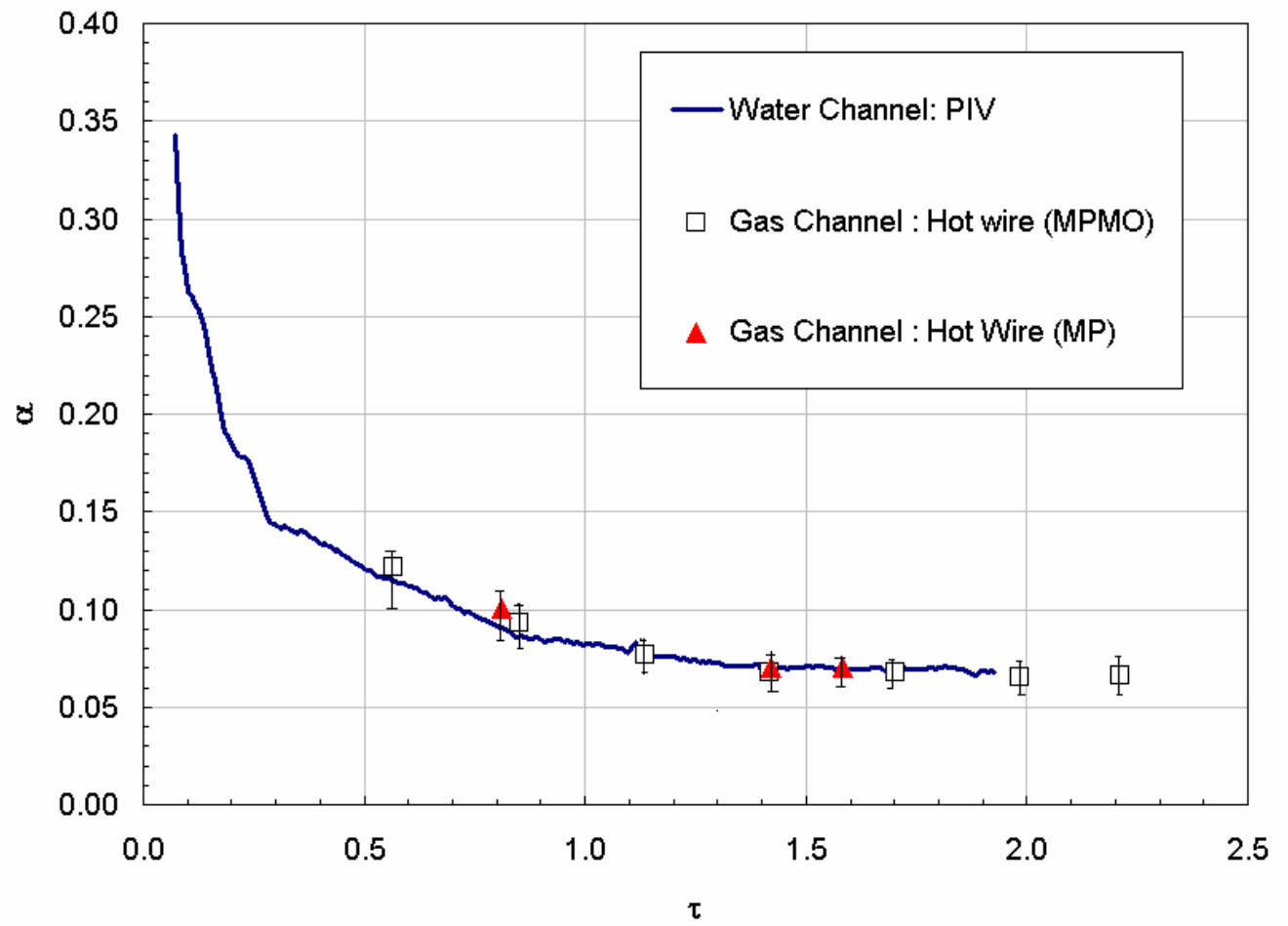

Figure 6.13 Comparison of measured $\alpha$ by different techniques (Hot-wire: MPMO: Multi-position Multi-overheat method; MP: Multi-position method) at $A_{\mathrm{t}} \# 0.04$. Water channel measurement was at $A_{\mathrm{t}} \# 7.5 \times 10^{-4}$. 
centerline of the mix, since at the center $f_{1}=f_{2}=0.5$ and $B_{2}=0.25$ according to Equation 6.8. It was noteworthy that all the parameters change little in the developing self-similar regime of the mix $(\tau>1)$.

The centerline root mean square values of the vertical, $v^{\prime}$ and horizontal, $u$ ' velocities were determined using this technique at 7 downstream locations (for $x=0.5 \mathrm{~m}$, $0.75 \mathrm{~m}, 1.0 \mathrm{~m}, 1.25 \mathrm{~m}, 1.5 \mathrm{~m}, 1.75 \mathrm{~m}$ and $1.95 \mathrm{~m})$. The results were tabulated in Table 6.3 . By using Equation 6.7 and the centerline value of v', we imply that the expansion of the mix was driven by velocity fluctuations that occur across the whole mix and not just the edge. The $v^{\prime}$ values grow linearly with time in the self-similar region. The growth constant $\alpha$ was evaluated by computing the ratio: $v^{\prime} /\left\{2 A_{t} g\left(x / U_{m}\right)\right\}$. The measured ratio at the 7 downstream locations was plotted as a function of non-dimensional time $\tau$ in Figure 6.13. It is found that these measurements are consistent with the measurement done with the multi-position single wire technique and also with the measurement of $\alpha$ done with the visualization analysis.

Measurements were also made at different locations across the mix at a downstream location of $x=1.75 \mathrm{~m}$ which corresponds to a $\tau$ of 1.986 . The centerline of the mix was found at the start of the run and the probe was moved in discrete steps $(\sim 9 \mathrm{cms})$ to measure various parameters at 9 different locations across the mix. As can be seen with Figure 6.10, the two end points are outside the mixing layer. Since our flow is a channel type flow, we measure a small value of $u^{\prime}$ at these locations. Figure 6.10 plots the velocity correlations across the mix at $x=1.75 \mathrm{~m}(\tau=1.986)$ for an experimental run at Atwood \# 0.04. Since there is no shear in this experiment, $\left\langle v^{\prime 2}\right\rangle$ dominates over 


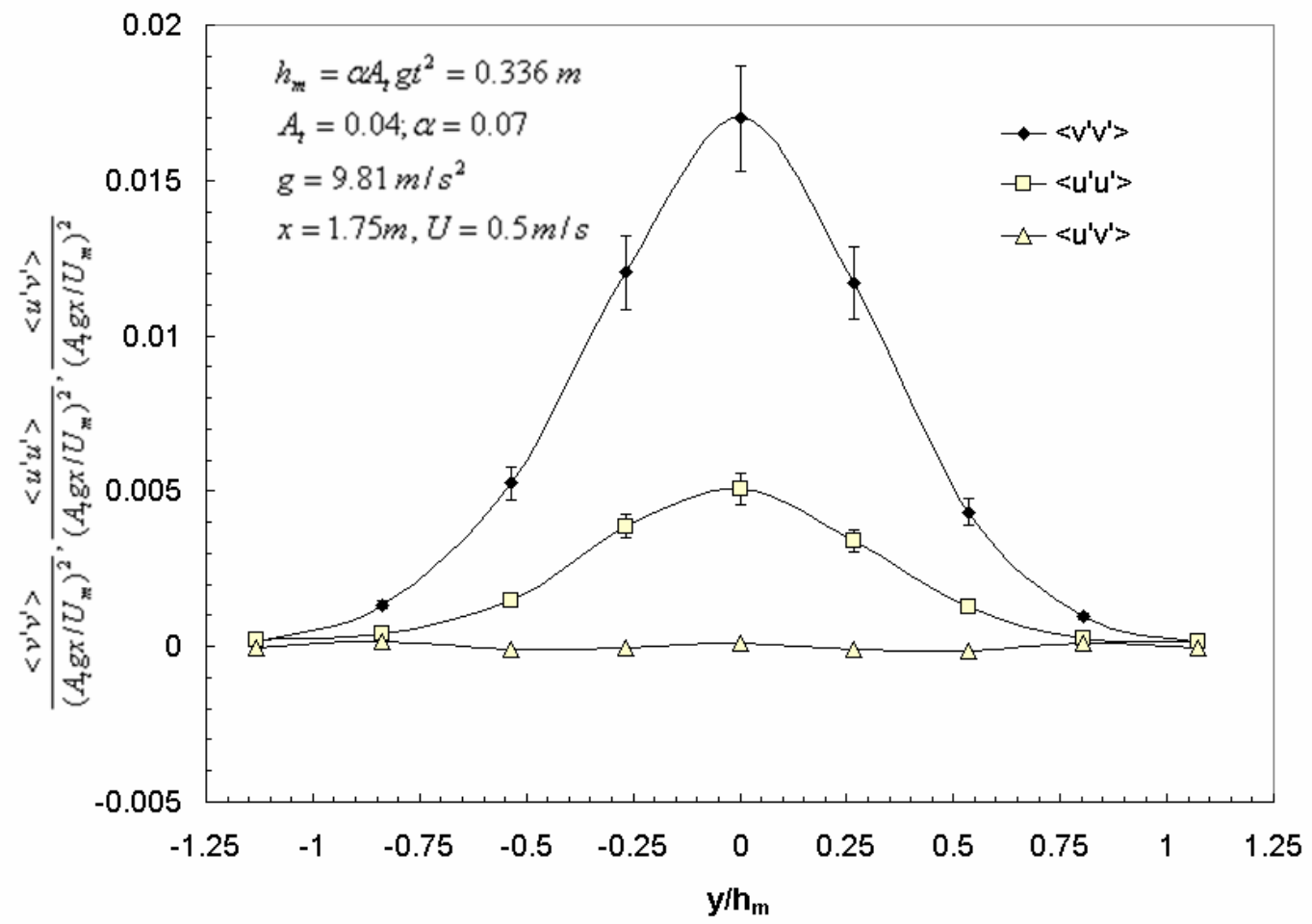

Figure 6.14 Velocity correlations across the mix at $x=1.75 \mathrm{~m}(\tau=1.986)$ for an experimental run at $A_{\mathrm{t}} \# 0.04$. (Note: The error in these measurements of $\langle u$ ' $v$ ' $\rangle$ nondimensionalized as shown is $\pm 2 \%$ ) 


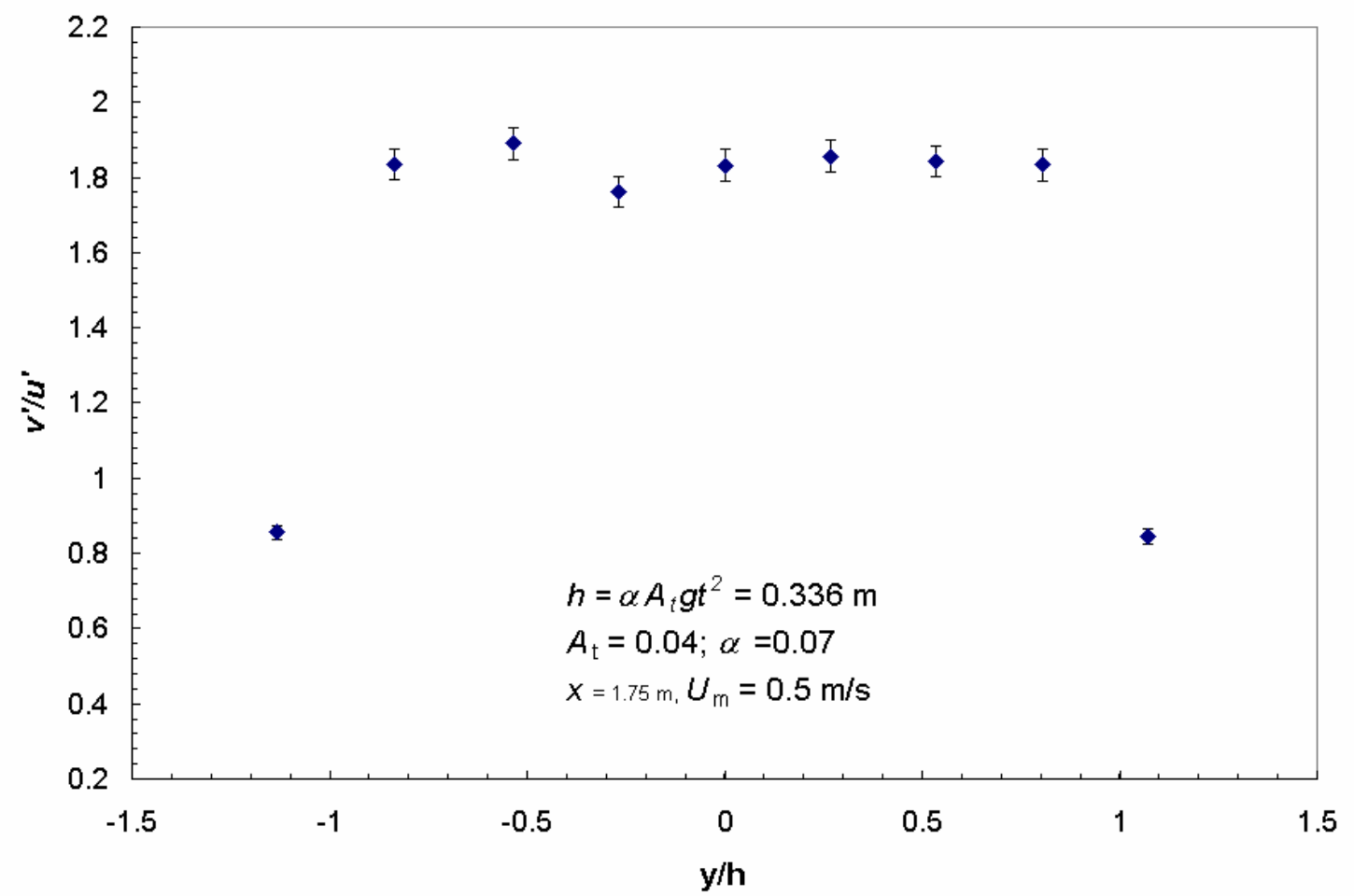

Figure 6.15 Ratio of $\frac{v^{\prime}}{u^{\prime}}$ across the mix at $x=1.75 \mathrm{~m}(\tau=1.986)$ for an experimental run at $A_{\mathrm{t}} \# 0.04$. 
$<u^{\prime 2}>$ everywhere across the mix. The cross correlation term $\overline{u^{\prime} v^{\prime}}$ was also found to be negligible since the mushroom-shaped structures have left-right symmetry about the center, so that $\left.u^{\prime} v^{\prime}\right|_{\text {right }}=-\left.u^{\prime} v^{\prime}\right|_{\text {left }}$ (Ramaprabhu \& Andrews 2004). Furthermore, the ratio $\frac{v^{\prime}}{u^{\prime}}$ is almost constant across the mixing layer as seen in Figure 6.14. The primary transport term in a R-T flow is the mass flux term $\left\langle\rho^{\prime} v>\right.$, which can be computed from the hot-wire data. The evolution of $\left\langle\rho^{\prime} v\right\rangle$ normalized by $\Delta \rho\left(A_{t} g x / U_{m}\right)$ is shown in Figure 6.15. It should be noted here that the term $A_{t} g x / U_{m}$ is the "free-fall velocity". It is seen that the mass flux term reaches a constant value when the flow becomes selfsimilar. Figure 6.16 shows the values of $\left\langle\rho^{\prime} v^{\prime}\right\rangle$ and $\left\langle\rho^{\prime} u>\right.$ across the mix at $\tau=1.986$. It is seen that $\left\langle\rho^{\prime} u\right\rangle$ is negligible across the mixing layer, due once again to the leftright symmetry of the mushroom-shaped structures, but $\left\langle\rho^{\prime} v>\right.$ has a peak that increases in magnitude with distance downstream. The vertical mass flux is negative because a packet of fluid that is lighter than its neighboring fluid particles $\left(\rho^{\prime} \equiv \rho-\bar{\rho}<0\right)$ will travel upwards with a velocity $v^{\prime}\left(v^{\prime}>0\right)$ and vice-versa, giving a negative correlation between $\rho^{\prime}$ and $v^{\prime}$. 


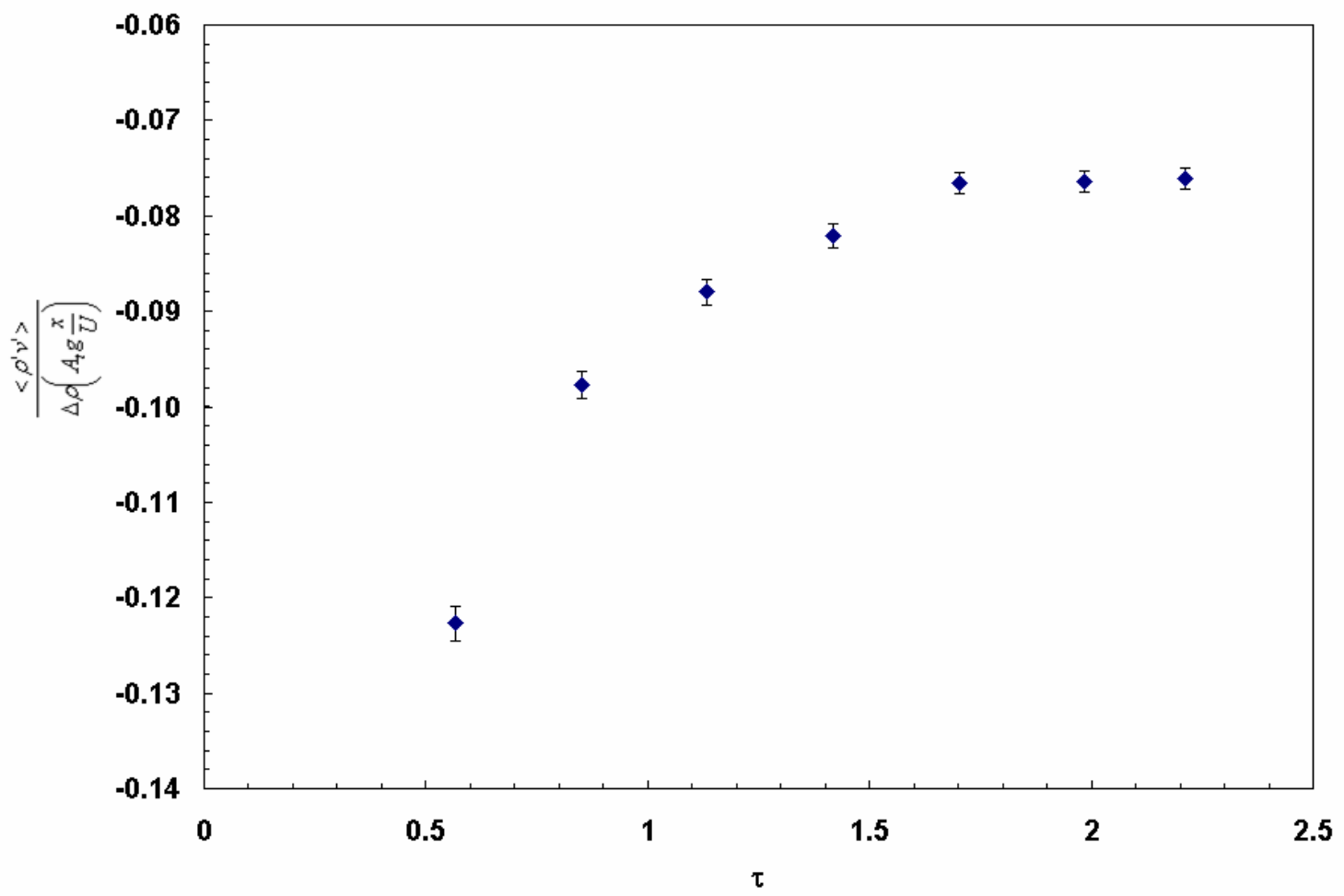

Figure 6.16 Evolution of primary transport term for $A_{\mathfrak{t}} \# 0.04$. 


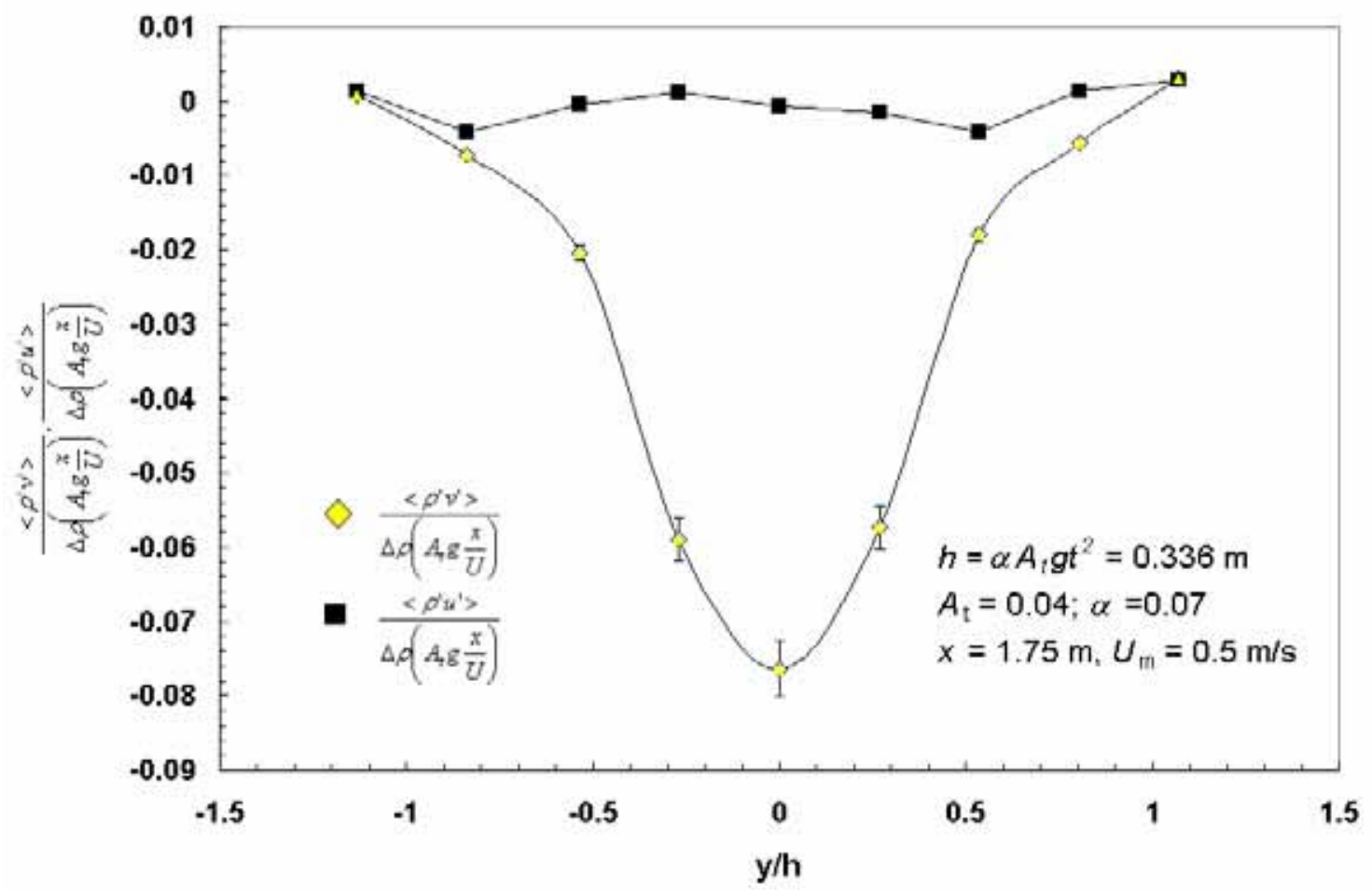

Figure 6.17 Profile of $\left\langle\rho^{\prime} v>\right.$ and $\left\langle\rho^{\prime} u>\right.$ across the mix at $\tau=1.986$ for $A_{\mathrm{t}} \# 0.04$ (Note: The error in these measurements of $\langle\rho$ ' $u$ ' $>$ non-dimensionalized as shown is $\pm 2.5 \%)$ 


\subsection{Energy Budget}

From our simultaneous measurements of velocity and density fields, the net kinetic energy dissipation from the initial state of the flow is calculated based on previously done similar calculations (Ramaprabhu \& Andrews 2004; Youngs 1984). The initial potential energy $P E_{i}$, associated with the flow, is calculated assuming a step-function at $z$ $=H / 2$ for the density profile at $T=0$. Thus,

$$
P E_{i}=\int_{0}^{H} \rho_{\text {step }} z d z=\int_{0}^{H / 2} \rho_{1} g z d z+\int_{H / 2}^{H} \rho_{2} g z d z
$$

Also, at $T=0, K E_{i} \sim 0$, since there is negligible energy associated with velocity fluctuations. Further downstream, the potential energy at $\tau=1.96$ is computed from the measured density profile as

$$
P E_{f}=\int_{0}^{H} \bar{\rho} g z d z \cong \sum_{i=0}^{n} \rho_{i} g z_{i} \Delta z
$$

The potential energy released to the flow by $T=1.96$ is then given by $P E_{\text {released }}=P E_{f}-$ $P E_{i}$. Some of this energy is converted into kinetic energy, which can be directly obtained from our measured velocity profiles of $u^{\prime}$ and $v^{\prime}$. Axisymmetric mushroom structures mean we may take the turbulence as homogeneous with respect to the stream-wise direction $x$ and the span-wise direction $z$, and set $\left\langle u^{\prime 2}\right\rangle=\left\langle w^{\prime 2}\right\rangle$. Then,

$$
K E_{\text {generated }}=\frac{1}{2} \int_{0}^{H} \rho\left(2<u^{\prime 2}>+<v^{\prime 2}>\right) d z
$$

and the net kinetic energy dissipation is given by 


$$
D=P E_{\text {released }}-K E_{\text {generated }}
$$

The net $K E$ dissipation as a fraction of the potential energy released, $D / P E_{\text {released }}$ was determined from our measurements to be 0.48 at $\tau \sim 1.96$. Ramaprabhu and Andrews (2004) obtained a value of 0.49 at $\tau \sim 1.21$. Youngs (1984) reported a value of 0.52 obtained from 3D numerical simulations. The value obtained from 2D simulations was significantly less $\left(D / P E_{\text {released }} \sim 0.06\right)$ since dissipation is primarily a $3 \mathrm{D}$ mechanism. For a self-similar mix, characterized by the length scale $g t^{2}$, it is expected that $D / P E_{\text {released }}$ and $K E_{\text {generated }} / P E_{\text {released }}$ become constant in the self-similar regime. Thus, we find good agreement between the present experiments and related, but higher Atwood number, 3D simulations. 


\section{CONCLUSIONS}

A novel air-helium gas channel experiment was constructed to study the development of high Atwood number Rayleigh-Taylor mixing. This dissertation reports experimental runs for low Atwood numbers from 0.035 to 0.25 . The experiment allows a heavy fluid to be placed over a light fluid, and a long duration to study the mixing process. Diagnostics used included constant temperature hot-wire anemometry and digital image analysis. A multi-position single-wire technique was used to measure the velocity fluctuations in three mutually perpendicular directions. However, this technique did not take into consideration the effect of Helium on the measurements. So an improvement over this technique, a multi-position multi-overheat technique was developed to measure various velocity, density and velocity-density correlations in the flow. Analysis of the measured data was used to explain the mixing as it develops to a self-similar regime in this flow and also to provide for a value of the growth parameter $\alpha$. The significant conclusions of this work are summarized below:

- In this experiment, the Rayleigh-Taylor mix, after a stage of linear growth, evolve through mode-interactions and successive wavelength saturation. The fully-developed mix grows quadratically in time, with a self-similar structure.

- Characterization of the mix width development was done using centerline vertical velocity fluctuations. The quadratic growth rate constant $\alpha$ was measured at 0.065 using hot-wire anemometry. This was further verified with an image analysis technique. The mixture fraction obtained from the visualization analysis 
suggests that the air and helium-air mixture were linearly distributed across the mixing layer. The value of $\alpha$ obtained by the visualization technique agreed well with that obtained by the hot-wire method. To verify that errors were indeed measurement errors and not as a result of lack of statistical convergence, a detailed convergence test was performed. The study revealed that the statistical convergence was a direct consequence of the number of different large 3D structures that were averaged over the duration of the run. These measurements good agreement with previous measurements from similar experiment (water channel) using the mix width based on the 5\% and 95\% threshold for the volume fractions. This verifies the fact that large-scale structures dominate the mixing and contain most of the kinetic energy of the mix.

- The vertical velocity fluctuations dominating the horizontal (stream-wise and transverse) components. The ratio of $v^{\prime} / u^{\prime}$ is $\sim 1.8$, and appears to be constant across the mix, as the mushroom-shaped structures are convected up and down the mix without much change in shape. The internal structure of the self-similar mix is thus anisotropic.

- From an energy budget analysis, it was shown that there is considerable kinetic energy dissipation ( $\sim 50 \%$ of the potential energy loss), which indicates the presence of highly three-dimensional structures necessary for mixing to occur.

- The molecular mix fraction, a local mix parameter, was determined to be $\sim 0.70$ in the self-similar region. The value of the mix fraction was found to be almost constant across the mix. Prandtl number and Schmidt number $\sim 1$ for the facility. 
- A major improvement in the exit plenum design was the introduction of an "exit splitter plate" in the exit section. A flap was also placed on the top half of the exit section and was located about 24 inches from the test section. Since helium is naturally buoyant, it was noticed that with an increase in the amount of helium in the bottom half of the channel (with increase in Atwood number), the center-line of the mix had a tendency to go up. To arrest this behavior and keep the centerline more or less horizontal, an exit splitter plate was introduced and a flap was placed on the top half of the channel. The opening of the flap was adjusted to keep the center-line of the mix more or less horizontal in end of the test section.

- A new convective heat transfer correlation was formulated for variable-density flows (binary gas mixture of air and helium) past a heated cylinder. The wire diameter was sufficiently small for the effects of molecular nature of the fluid to be experienced. Molecular effects take the form of changed boundary conditions as compared with continuum flow - there was a jump in temperature between the surface of the wire and the fluid adjacent to it. A continuum Nusselt number was evaluated and the calibration data non-dimensionalized to obtain a working correlation for variable density flows consisting of air-helium mixture. However, because of the low velocities used in the experiment $\left(U_{\mathrm{m}}<2 \mathrm{~m} / \mathrm{s}\right)$, the correlation was limited to small Reynolds $(R e)$ numbers. Some scatter was observed in the low Re regime which may be attributed to combined effects of forced and natural convection. 
- The multi-position multi-overheat method an "time-averaged" technique and was initially thought to be robust enough to measure the various statistics for a wide range of Atwood numbers for the flow. However, there are some major limitations with this technique to be used in high Atwood number runs $\left(A_{\mathrm{t}}>\right.$ 0.25). As described in section 4.3.2, the technique assumes a value of $d E / d \rho$ for the entire density during the run. In practice, this is accurate if the response of $E$ vs. $\rho$ is linear, which is the case for small density difference $\left(A_{\mathrm{t}} \sim 0.25\right)$. A value of $d E / d \rho$ thus selected encompasses the entire range of densities accurately. However, for large density differences $\left(A_{\mathrm{t}}>0.25\right)$, the response becomes significantly non-linear as can be seen in Figures 4.11 and 4.16. Using a linear assumption (by calculating derivatives/sensitivities) would result in significant errors in the measured quantities. Thus this method was not attempted for any high Atwood measurements. The alternate technique to thus measure all densityvelocity correlations as well as evaluate spectral information is to pursue an "instantaneous" technique by using a combination of a 3 wire probe (operated in CTA mode) with a cold wire probe (operated in CCA mode) placed very close to it. The cold-wire, which works as a resistance wire will measure instantaneous density during a run. This information can then be used to correlate and solve for the three-dimensional velocity fluctuations on an instantaneous basis. This is left as a future work. 


\section{LITERATURE CITED}

Acrivos A. 1966. On the combined effect of forced and free convection heat transfer in laminar boundary layer flows. Chem. Eng. Sci. 21:343-52

Aihara Y, Kassoy DR, Libby PA. 1967. Heat transfer from cylinders at low Reynolds numbers. II. Experimental results and comparison with theory. Phys. Fluids 10:947-52

Allred JC, Blount GH. 1954. Experimental studies of Taylor instability, University of California report LA-I.600

Almquist P, Legath E. 1965. The hot-wire anemometer at low air velocities. DISA Info 2:3-4

Andrews GE, Bradley D, Hundy GF. 1972. Hot-wire anemometer calibration for measurements of small gas velocities. Int. J. Heat Mass Trans. 15:1765-86

Andrews MJ. 1986. Turbulent mixing by Rayleigh-Taylor instability. London: Imperial College

Andrews MJ, Spalding DB. 1990. A simple experiment to investigate two-dimensional mixing by Rayleigh-Taylor instability. Phys. Fluids A 2:922-27

Anuchina NN, Kucherenko YA, Neuvazhaev VE, Ogibina VN, Shibarshov LI, et al. 1978. Turbulent mixing at an accelerating interface between liquids of different densities. Translated from Izv. Akad Nauk. SSSR, Mekh. Zhidk. Gaza 6:157-60

Atzeni S, Meyer-ter-Vehn J. 2004. The physics of inertial fusion: beam plasma interaction, hydrodynamics, hot dense matter. In International Monographs on Physics; ed. J Birman, SF Edwards, R Friend, M Rees, D Sherrington, G Veneziano, pp. 1-458. Oxford: Oxford University Press

Baid KM. 1967. Measurement of velocity in gas mixtures. MS thesis. Illinois Institute of Technology, Chicago

Banerjee A, Andrews MJ. 2006. Statistically steady measurements of Rayleigh-Taylor mixing in a gas channel. Phys. Fluids 18:1-13

Besnard D, Harlow FH, Rauenzahn R, Zemach C. 1990. Spectral transport model for turbulence. Los Alamos Report, LA-11821 
Besnard D, Harlow FH, Rauenzahn R, Zemach C. 1992. Turbulence transport equations for variable density turbulence, and a relationship to two-fluid models. Los Alamos Report, LA-11303

Betti R, Umansky M, Lobatchev V, Goncharov VN, McCrory RL. 2001. Hot-spot dynamics and deceleration-phase Rayleigh-Taylor instability of imploding inertial confinement fusion capsules. Phys. Plasmas 8:5257-67

Brouillette M. 2002. The Richtmyer-Meshkov instability. Annu. Rev. Fluid Mech. $34: 445-68$

Browand FK, Weidman PD. 1976. Large scales in the developing mixing layer. J.Fluid Mech. 76:127-44

Brown GL, Roshko A. 1974. On density effects and large structures in turbulent mixing layers. J. Fluid Mech. 64:775-816

Bruun HH. 1972. Hot-wire corrections in low and high turbulence intensity flows. $J$. Phys. E: Sci. Instr. 5:812-18

Bruun HH. 1996. Hot-wire anemometry : principles and signal analysis. Oxford: Oxford University Press

Bruun HH, Tropea C. 1985. The calibration of inclined hot-wire probes. J. Phys. E: Sci. Inst. 18:405-13

Chandrasekhar S. 1961. Hydrodynamic and hydromagnetic stability. New York: Dover

Chassaing P, Harran G, Joly J. 1994. Density fluctuations in free turbulent binary mixing. J. Fluid Mech. 279:239-78

Chen TS, Mucoglu A. 1975. Buoyancy effects on forced convection along a vertical cylinder. J. Heat Trans. 97C:198-203

Clarke JS, Fisher HN, Mason RJ. 1973. Laser-driven implosion of spherical DT targets to thermonuclear burn conditions. Phys. Rev. Lett. 30:89-92

Cole J, Roshko A. 1954. Heat transfer from wires at Reynolds numbers in the Oseen range. Proc. Heat Trans. Fluid Mech. Inst., University of California, Berkeley. $6: 357-84$

Cole RL, Tankin RS. 1973. Experimental study of Taylor instability. Physics of Fluids 16:1810-15 
Collis DC, William MJ. 1959. Two-dimensional convection from heated wires at low Reynolds numbers. J. Fluid Mech. 6:357-84

Comte-Bellot G. 1976. Hot-wire anemometry. Ann. Rev. Fluid Mech. 8:209-31

Cook AW, Cabot W, Miller PL. 2004. The mixing transition in Rayleigh-Taylor instability. J. Fluid Mech. 511:333-62

Cook AW, Dimotakis PE. 2001. Transition stages of Rayleigh-Taylor instability between miscible fluids. J. Fluid Mech. 443:69-99

Corrisin S. 1949. Extended applications of the hot-wire anemometer, Rev. Sci. Instr. $18: 469-71$

Cui AQ, Street RL. 2004. Large-eddy simulation of coastal upwelling flow. Env. Fluid Mech. 4:197-223

Dalziel SB, Linden PF, Youngs DL. 1999. Self-similarity and internal structure of turbulence induced by Rayleigh-Taylor instability. J. Fluid Mech. 399:1-48

Danckwerts PV. 1952. The definition and measurement of some characteristics of mixtures. Appl. Sci. Res 3:279-96

Deissler RG. 1964. Analysis of second-order slip flow and temperature jump boundary conditions for rarefied gas. Int. J. Heat Mass Trans. 7:681-94

Dimonte G. 1999. Nonlinear evolution of the Rayleigh-Taylor and Richtmyer-Meshkov instabilities. Phys. Plasmas 6:2009-15

Dimonte G, Schneider M. 1996. Turbulent Rayleigh-Taylor instability experiments with variable acceleration. Phys. Rev. E 54:3740-43

Dimotakis PE. 2000. The mixing transition in turbulent flows. J.Fluid Mech. 409:69-98

DiPrima RC, Swinney HL. 1981. Hydrodynamic instabilities and the transition to turbulence. Berlin: Springer.

Emmons HW, Chang CT, Watson BC. 1960. Taylor instability of finite surface waves. $J$. Fluid Mech. 7:177-93

Ezekwe CI, Pierce FJ, McAllister JE. 1978. Measured Reynolds stress tensors in a threedimensional turbulent boundary layer. AIAA J. 16:645-46 
Gebhart B, Pera L. 1970. Mixed convection from long horizontal cylinders. J.Fluid Mech. 45:1-49

Goldstein RJ. 1996. Fluid mechanics measurements. New York:Hemisphere Publishing Corporation

Gull SF. 1975. The X-ray, optical and radio properties of young supernova remnants. Royal Astronom. Soc. Monthly Notices. 171:263-78

Harion JL, Marinot MF, Camano B. 1996. An improved method for measuring velocity and concentration by thermo-anemometry in turbulent helium-air mixtures. Exp. Fluids 22:174-82

Hinze JO. 1959. Turbulence: an introduction to its mechanism and theory: New York: McGrawHill

Jacobs JW, Caton I. 1988. Three-dimensional Rayleigh-Taylor instability. Part 2 Experiment. J.Fluid Mech.187:353-71

Jacobs JW, Dalziel SB. 2005. Rayleigh-Taylor instability in complex stratification. $J$. Fluid Mech. 542:251-79

Jitschin W, Weber U, Hartmann HK. 1995. Convenient primary gas flow meter. Vacuum 46:821-25

John JEA. 1984. Gas dynamics. New York: Prentice Hall

Jørgensen FE. 1996. The computer-controlled constant temperature anemometer: aspects of set-up, probe calibration, data acquisition and data conversion. Meas. Sci. Tech. 7:1378-87

Kaminsky M. 1965. Atomic and ionic impact phenomena on metal surfaces. Berlin: Springer

Kanevce G, Oka S. 1973. Correcting hot-wire readings for influence of fluid temperature variations. DISA Info. 15:21-4

Kassoy DR. 1967. Heat transfer from circular cylinders at low Reynolds numbers. I. Theory for variable property flow. Phys. Fluids 10:938-46

Kennard EH. 1938. Kinetic theory of gases. New York:McGraw Hill 
King LV. 1914. On the convection of heat from small cylinders in a stream of fluid: determination of the convection constants of small platinum wires with applications to hot-wire anemometry. Phil. Trans. Royal Soc. A214:373-432

Kline SJ, McClintock FA. 1953. Describing uncertainties in single-sample experiments. Mech. Eng. 75:3-8

Koop GK. 1976. Instability and turbulence in a stratified shear layer. $\mathrm{PhD}$ dissertation. University of Southern California, Los Angeles

Koppius AM, Trines GRM. 1976. The dependence of hot-wire calibration on gas temperature at low Reynolds numbers. Int. J. Heat Mass Trans. 19:967-74

Kovasznay LSG. 1950. The hot-wire anemometer in supersonic flow. J.Aero.Sci. $17: 565-72$

Kucherenko YA, Balabin SI, Ardashova RI, Kozelkov OE, Dulov AV, Romanov IA. 2003. Experimental study of the influence of the stabilizing properties of transitional layers on the turbulent mixing evolution. Las. Part. Beams 21:369-73

Kucherenko YA, Balabin SI, Cherrey R, Haas JF. 1997. Experimental investigation into inertial properties of Rayleigh-Taylor turbulence. Las. Part. Beams 15:25-31

LaRue JC, Deaton T, Gibson CH. 1975. Measurement of high-frequency turbulent temperature. Rev. Sci. Instr. 46:757-64

Lee T, Budwig R. 1991. Two improved methods for low-speed hot-wire calibration. Meas. Sci. Tech. 1:643-46

Lewis DJ. 1950. The instability of liquid surfaces when accelerated in a direction perpendicular to their planes II. Proc. Royal Soc. London A 202:81-96

Linden PF, Redondo JM, Caulfield CP. 1992. Molecular mixing in Rayleigh-Taylor instability. In Advances in compressible turbulent mixing, ed. WP Dannevik, AC Buckingham, CE Leith, pp. 95-104

Lindl JD. 1998. Inertial confinement fusion: the quest for ignition and energy gain using indirect drive. Berlin: Springer

Loehrke RI, Nagib HM. 1972. Experiments on management of free-stream turbulence. AGARD Report 598, Illinois Institute of Technology, Chicago

Manca O, Mastullo R, Mazzei P. 1988. Calibration of hot-wire probes at low velocities in air with variable temperature. Dantec Info. 6:6-8 
Marmottant P, Villermaux E. 2004. On spray formation. J.Fluid Mech. 498:73-111

Mason EA, Saxena SC. 1958. Approximate formula for the thermal conductivity of gas mixtures. Phys. Fluids 1:361-69

McAdams WH. 1954. Heat transmission. New York: McGraw-Hill

McCarty R. 1973. Thermodynamic properties of helium 4 from 2 to $1500 \mathrm{~K}$ at pressures up to 108 Pa. J. Phys. Chem. Ref. Data 2:923-1042

Meshkov EE. 1969. Instability of the interface of two gases accelerated by a shock wave. Sov. Fluid Dyn. 4:101-108

Molchanov OA. 2003. On the origin of low- and middler-latitude ionospheric turbulence. Phys. Chem.Earth 29:559-67

Mueschke N. 2004. An investigation on the influence of initial conditions on RT mixing. MS thesis. Texas A\&M University, College Station

Mueschke N, Andrews MJ. 2005. Investigation of scalar measurement error in diffusion and mixing process. Exp. Fuids 40:165-76

Pierce FJ, Ezekwe CI. 1976. Comparison of Reynolds stress diagnostics by fixed and rotating probes. AIAA J. 14:412-14

Pitts WM, McCaffrey BJ. 1986. Response behavior of hot-wires and films to flows of different gases. J. Fluid Mech. 169:465-512

Popil R, Curzon FL. 1979. Production of reproducible Rayleigh-Taylor instabilities. Rev. Sci. Instr. 50:1291-95

Preckshot GW, Gorman JW. 1958. Steady state longitudinal and radial temperature distribution in internally heated finite wires. Ind. Eng. Chem. Res. 50:837-48

Ramaprabhu P, Andrews MJ. 2003. Simultaneous measurement of velocity and density in buoyancy driven mixing. Exp. Fluids 34:98-106

Ramaprabhu P, Andrews MJ. 2004. Experimental investigation of Rayleigh- Taylor mixing at small Atwood numbers. J. Fluid Mech. 502:233-71

Ratafia M. 1973. Experimental investigation of Rayleigh-Taylor instability. Phys. Fluids $16: 1207-10$ 
Rayleigh L. 1884. Investigation of the equilibrium of an incompressible heavy fluid of variable density. Proc. London Math. Soc. 14: 170-77

Read KI. 1984. Experimental investigation of turbulent mixing by Rayleigh-Taylor instability. Physica D 12:45-58

Richtmyer RD. 1960. Taylor Instability in shock acceleration of compressible fluids. Comm. Pure Appl. Math. 8:297-319

Roberts PD, Rose SJ, Thompson PC, Wright RJ. 1980. The stability of multiple shell ICF targets. J. Phys D : Appl. Phys. 13:1957-69

Rose WC. 1973. The behavior of a compressible turbulent boundary layer in a shockwave-induced adverse pressure gradient. $\mathrm{PhD}$ dissertation. University of Washington, Seattle

Saddoughi SG, Veeravalli SV. 1996. Hot-wire anemometry behavior at very high frequencies. Meas. Sci.Tech. 7:1297-300

Simpson RL, Wyatt WG. 1973. The behaviour of hot-film anemometers in gas mixtures. J. Phys. E: Sci. Instr.6:981-87

Smarr L, Wilson JR, Barton RT, Bowers RL. 1981. Rayleigh-Taylor overturn in supernova core collapse. Astrophys.J. 246:515-25

Smith WJ. 2000. Modern optical engineering. New York: McGraw Hill

Snider DM. 1994. A study of buoyancy and shear mixing. PhD dissertation. Texas A\&M University, College Station

Snider DM, Andrews MJ. 1994. Rayleigh-Taylor and shear driven mixing with an unstable thermal stratification. Phys. Fluids 6:3324-34

Snider DM, Andrews MJ. 1995. An inexpensive method for quantitative data collection from photographic prints. Meas. Sci. Tech. 6:502-6

Snider DM, Andrews MJ. 1996. The simulation of mixing layers driven by compound buoyancy and shear. J.Fluids Eng. 118:370-76

Stanford RA, Libby PA. 1974. Further applications of hot-wire anemometry to turbulence measurements in helium-air mixtures. Phys. Fluids 17:1353-61

Steinkamp MJ, Clark T, Harlow FH. 1995. Stochastic interpenetration of fluids. Los Alamos Report, LA-131016 
Stillinger DC, Head MJ, Helland KN, Van Atta CW. 1983. A closed loop gravity driven water channel for density stratified flow. J. Fluid Mech. 131:73-89

Swaminathan MK, Rankin GW, Sridhar K. 1986. Evaluation of the basic systems of equation for turbulence measurements using the Monte Carlo technique. J.Fluid Mech. 170:1-19

Tan-Atichat J, Nagib HM, Loehrke RI. 1982. Interaction of free-stream turbulence with screens and grids: a balance between turbulence scales. J.Fluid Mech.114:501-28

Taylor GI. 1938 The spectrum of turbulence. Proc. Royal Soc. London Ser. A 164:47690

Taylor GI. 1950. The instability of liquid surfaces when accelerated in a direction perpendicular to their planes I. Proc.Royal Soc. London Ser.A 201:192-96

Tennekes H, Lumley J. 1994. A first course in turbulence. Cambridge: MIT Press

Thomas GO. 2003. The aerodynamic breakup of ligaments. Atom. Sprays 13:117-29

Van der Hegge Zijnen BG. 1956. Modified correlation formulae for heat transfer by natural and forced convection from horizontal cylinders. Appl. Sci.Res.6:129-40

Wasan DT, Baid KM. 1971. Measurement of velocity in gas mixtures: hot-wire and hotfilm anemometry. AiChE J. 17:729-31

Way J, Libby PA. 1970. Hot-wire probes for measuring velocity and concentration in helium and air mixture AIAA J. 8:976-78

Way J, Libby PA. 1971. Application of hot-wire anemometry and digital techniques to measurements in a turbulent helium jet. AIAA J. 9:1567-73

Wilke CR. 1950. A viscosity equation for gas mixtures. J. Chem. Phys. 18:517-19

Wilson PN. 2002. A study of buoyancy and shear driven turbulence within a closed water channel. PhD dissertation. Texas A\&M University, College Station

Wilson PN, Andrews MJ, Harlow F. 1999. Spectral non-equilibrium in a turbulent mixing layer. Phy. Fluids A 11:2425-33

Wilson PN, Andrews PN. 2002. Spectral measurements of Rayleigh-Taylor mixing at small Atwood number. Phys. Fluids A 14:938-45 
Wu B, Molinas A. 2001. Choked flows through short contractions. J. Hydr. Eng. 127:657-62

Wu P, Libby PA. 1971. Heat transfer to cylinders in helium and helium-air mixtures. Int. J. Heat .Mass Tran. 14:1071-77

Youngs DL. 1984. Numerical simulation of turbulent mixing by Rayleigh-Taylor instability. Physica D 12:32-44

Youngs DL. 1989. Modelling turbulent mixing by Rayleigh-Taylor instability. Physica D 37:270-87 


\section{APPENDIX A}

\section{DESIGN BASIS}

\section{A.1 Design Basis of the Experiment}

In the experiment of buoyant mixing with a stream-wise velocity, $U_{\mathrm{m}}$, the requirement was that downstream behavior does not affect upstream mixing. Unlike shear layers which grow linearly with distance, buoyancy mixing was approximately parabolic and grows as axial distance squared. For an average fluid quantity, $\bar{\varphi}=\frac{1}{T} \int_{0}^{T} \varphi d t$, the steady two dimensional equation of motion can be illustrated as:

$$
U_{m} \frac{\partial \bar{\varphi}}{\partial x}+\bar{v} \frac{\partial \bar{\varphi}}{\partial y}=\Gamma\left(\frac{\partial^{2} \varphi}{\partial x^{2}}+\frac{\partial^{2} \varphi}{\partial y^{2}}\right)
$$

where $U_{m}$ was the convective velocity, $\bar{v}$ was the cross stream mingling velocity and $\Gamma$ was the turbulent plus molecular diffusion constant. Since R-T experiments conducted at our facility has shown that $\bar{v} \sim 0$, the $2^{\text {nd }}$ term drops out. Defining $x=U_{m} t$, Equation A.1 simplifies into:

$$
\frac{\partial \bar{\varphi}}{\partial t}=\Gamma\left(\frac{1}{U_{m}^{2}} \frac{\partial^{2} \varphi}{\partial t^{2}}+\frac{\partial^{2} \varphi}{\partial y^{2}}\right)
$$

This was approximately parabolic if:

$$
\frac{1}{U_{m}^{2}} \frac{\partial^{2} \varphi}{\partial t^{2}}<<\frac{\partial^{2} \varphi}{\partial y^{2}}
$$


Consider $\bar{\varphi}$ to be the volume fraction, $f$. If $h$ was the half width of the mixing region, then $f$ for the upper half of the mixing layer may be approximated with a boundary layer profile:

$$
f=-\frac{y^{2}}{2 h^{2}}+\frac{y}{h}+0.5
$$

At $y=0$ (interface), $f=0.5$ and at $y=h, f=1.0$. Assuming that the mixing layer grows like a parabola, we substitute all relations into the PDE and get

$$
U_{m}^{2}>>4 \alpha^{\prime 2} t^{2}=\left(\frac{d h}{d t}\right)^{2}
$$

We then apply Taylor's Hypothesis (Taylor 1938) to convert the temporal derivative into a spatial one. Noting that $\left(\frac{d h}{d t}\right)=U_{m}\left(\frac{d h}{d x}\right)=U_{m} \tan \theta, \operatorname{then}(\tan \theta)^{2}<<1$. A maximum slope on the mixing width of 0.3 will give the approximate parabolic flow for the buoyant mixing layer. The relation between $U_{m}$, the mean convective velocity and $A_{t}$, the Atwood Number was thus:

$$
\sqrt{\frac{h \alpha g A_{t}}{U_{m}{ }^{2}}}<\frac{1}{3}
$$

Thus for any given Atwood number, the minimum flow velocity which will satisfy the parabolic approximation was tabulated in Table A.1. 
TABLE A.1 Minimum flow velocity to satisfy parabolic approximation in gas channel

\begin{tabular}{|c|c|c|}
\hline Atwood Number & Minimum $\boldsymbol{U}_{\mathbf{m}}(\mathbf{m} / \mathbf{s})$ & $\boldsymbol{U}_{\mathbf{m}}$ for $\boldsymbol{\theta}=\mathbf{1 5}^{\mathbf{0}} \mathbf{( m / s )}$ \\
\hline 0.05 & 0.48 & 0.54 \\
\hline 0.1 & 0.68 & 0.76 \\
\hline 0.25 & 1.07 & 1.21 \\
\hline 0.5 & 1.51 & 1.72 \\
\hline 0.75 & 1.85 & 2.09 \\
\hline
\end{tabular}




\section{A.2 Gravity Current Effects In The Gas Channel}

During initial high Atwood runs $\left(A_{\mathrm{t}}>0.1\right)$, we witnessed problems in starting the channel. This was attributed to a combination of the following factors:

(a) The bottom stream consisting of a lighter mass of air-helium mixture had to push out a heavier mass of air sitting in the test section. To eliminate this, the channel was initially filled with helium and a fan was used to circulate the air inside the channel test section. This helped to reduce the density of the fluid inside the channel. However, at high Atwood number runs $\left(A_{\mathfrak{t}} \sim 0.25\right.$ and higher), it was important that the channel be started at a velocity higher than the calculated $U_{\mathrm{m}}$. Once the denser mass of air in the test section was pushed out, the dampers can be adjusted to set the velocity back to the prescribed mean velocity.

(b) Since helium is naturally buoyant, it was noticed that with an increase in the amount of helium in the bottom half of the channel (with increase in Atwood number), the center-line of the mix had a tendency to go up. To arrest this behavior and keep the center-line more or less horizontal, an exit splitter plate was introduced and a flap was placed on the top half of the channel. The opening of the flap was adjusted to maintain a flat center-line towards the end of the channel.

(c) Gravity currents were found to induce a backflow in the Gas Channel. The exit plenum was redesigned and rebuilt to eliminate the gravity current to flow back into the test sections. A calculation in a square box provides a reasonable estimate of the effects of the gravity current. 
For Configuration 1, the initial Potential Energy, $\mathrm{PE}_{1}$, was given as:

$$
P E_{1}=\int_{0}^{L} \rho_{1} g \frac{L}{2} z d z+\int_{0}^{L} \rho_{2} g \frac{L}{2} z d z=g \frac{L^{3}}{4}\left(\rho_{1}+\rho_{2}\right)
$$

For Configuration 2, the final Potential Energy, $\mathrm{PE}_{\mathrm{f}}$, was given as:

$$
P E_{2}=\int_{0}^{\frac{L}{2}} \rho_{1} g L z d z+\int_{\frac{L}{2}}^{L} \rho_{2} g L z d z=g \frac{L^{3}}{8}\left(\rho_{1}+3 \rho_{2}\right)
$$

The Potential Energy converted to Kinetic Energy was given by:

$$
K E=P E_{1}-P E_{2}=g \frac{L^{3}}{4}\left(\rho_{1}+\rho_{2}\right)-g \frac{L^{3}}{8}\left(\rho_{1}+3 \rho_{2}\right)=g \frac{L^{3}}{8}\left(\rho_{1}-\rho_{2}\right)
$$

The KE in terms of the mean value of the velocity was:

$$
K E=\frac{1}{2}\left(\rho_{1}+\rho_{2}\right) \frac{L^{2}}{2} v^{2}
$$

Equating Equations B.3a and B.3b, we get :

$$
v^{2}=\frac{g L}{2}\left(\frac{\rho_{1}-\rho_{2}}{\rho_{1}+\rho_{2}}\right)=\frac{A_{t} g L}{2}
$$

Using a similar analogy for the Gas Channel and using $L=h=60 \mathrm{~cm}$, the vertical velocity component of the gravity wave falling downwards in calculated for the various run conditions in the channel (see Table A.2). This implies that for the low Atwood run (i.e. $\left.A_{\mathrm{t}} \# 0.05\right)$, the heavy air at the bottom should flow out at a velocity $=0.54-0.38=$ $0.16 \mathrm{~m} / \mathrm{s}$. To facilitate this process, a flexible ducting connected to a bottom suction fan was introduced into the test section to draw out the heavy mass of air. Once the heavy mass was drawn out, the flexible ducting was withdrawn and the fan was switched off. 


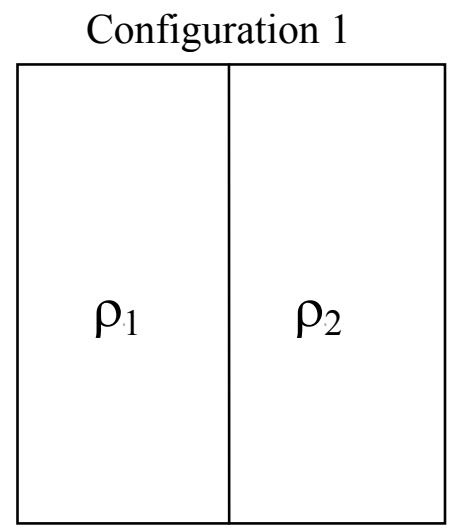

Configuration 2

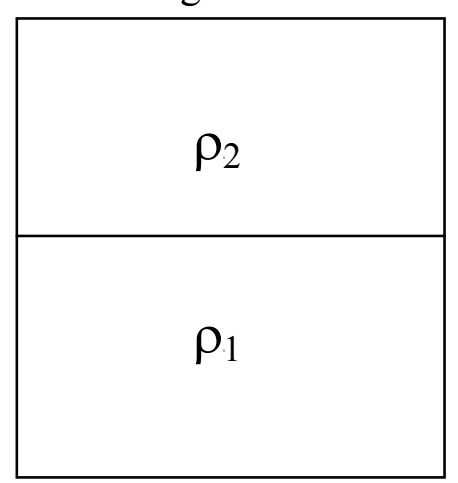

Figure A.1 Stable configurations $\left(\rho_{1}>\rho_{2}\right)$ in a square box $(1 \mathrm{~m} \times 1 \mathrm{~m})$ used to calculate conversion from potential energy to kinetic energy. 
TABLE A.2 Vertical velocity $(v)$ of the gravity wave and mean flow velocity $U_{\mathrm{m}}$ corresponding to different run conditions at a different Atwood numbers $(L=0.6 \mathrm{~m})$

\begin{tabular}{||c|c|c|c|c|}
\hline \multirow{2}{*}{ Facility } & Atwood Number & $\boldsymbol{v}(\mathbf{m} / \mathbf{s})$ & $\boldsymbol{U}_{\mathbf{m}}(\mathbf{m} / \mathbf{s})$ & $\rho_{2} U_{\text {mean }}^{2}-\rho_{1} v^{2}$ \\
\hline \multirow{3}{*}{$\begin{array}{c}\text { Gas } \\
\text { Channel }\end{array}$} & 0.05 & 0.383 & 0.54 & 0.142 \\
\cline { 2 - 5 } & 0.10 & 0.542 & 0.76 & 0.231 \\
\cline { 2 - 5 } & 0.25 & 0.858 & 1.21 & 0.175 \\
\cline { 2 - 5 } & 0.50 & 1.213 & 1.72 & -0.578 \\
\hline \multirow{2}{*}{$\begin{array}{c}\text { Water } \\
\text { Channel }\end{array}$} & 0.75 & 1.486 & 2.09 & -1.941 \\
\hline \hline
\end{tabular}




\section{APPENDIX B}

\section{ERROR ANALYSIS}

\section{B.1 Error in Mass Flow Rate Measurement}

For the experiment, the helium flow rates required for the entire range of Atwood Numbers $(0.035-0.75)$ varied from $0.0066 \mathrm{lbm} / \mathrm{s}$ to $0.282 \mathrm{lbm} / \mathrm{s}$. To meet these flow requirements, a wide range of orifice sizes $(d \sim 0.033 "-0.25$ ”) were calibrated. The least count of the scale used for mass calibration was $\pm 0.01 \mathrm{lbs}$. Since a bottle full of compressed helium ( $2100 \mathrm{psig})$ was about $1.5 \mathrm{lbm}$ heavier than an empty bottle (< 550psig), multiple bottles were used to ensure that the entire calibration run lasts at least 30 seconds. Hence the uncertainty in mass flow rate was evaluated as $w_{\dot{m}_{H e}}=0.01 / 30=$ $1.532 \times 10^{-4} \mathrm{~kg} / \mathrm{s}$.

\section{B.2 Error in Velocity Measurement}

As described in Section 4, a proportioner meter (Aalborg, Inc) was used for calibrating the hot-wire probes. The proportioner meter has two tubes which were separately calibrated for air and helium flow-rates. Each tube has 150 printed volume graduations along the length of the tube and can read upto a maximum volumetric flowrate of $1.1677 \times 10^{-3} \cdot \mathrm{m}^{3} / \mathrm{s}$ for air and $1.1685 \times 10^{-3} \cdot \mathrm{m}^{3} / \mathrm{s}$ for helium. Based on our velocity requirements, we used a $1 / 2$ " PVC pipe for calibration. The uncertainty of velocities was obtained based on the diameter of the pipe used and a value of $w_{U_{m}}=0.0615 \mathrm{~m} / \mathrm{s}$ was taken for the $1 / 2 "$ pipe. 


\section{B.3 Error in Atwood Number}

A Kline-McClintock Uncertainty Analysis (1953) was performed on Equation B.1:

$$
A_{t}=\frac{\rho_{\text {air }}-\rho_{\text {mix }}}{\rho_{\text {air }}+\rho_{\text {mix }}}
$$

The uncertainty in the Atwood number was defined as:

$$
\begin{gathered}
w_{A_{t}}=\left[\left(\frac{\partial A_{t}}{\partial \rho_{\text {air }}} \times w_{\rho_{\text {air }}}\right)^{2}+\left(\frac{\partial A_{t}}{\partial \rho_{\text {mix }}} \times w_{\rho_{\text {amix }}}\right)^{2}\right]^{\frac{1}{2}} \\
=\left[\left(\frac{2 \rho_{\text {mix }}}{\left(\rho_{\text {air }}+\rho_{\text {mix }}\right)^{2}} \times w_{\rho_{\text {air }}}\right)^{2}+\left(\frac{-2 \rho_{\text {air }}}{\left(\rho_{\text {air }}+\rho_{\text {mix }}\right)^{2}} \times w_{\rho_{\text {amix }}}\right)^{2}\right]^{\frac{1}{2}} \\
\rho_{\text {mix }}=\rho_{\text {air }}+\frac{\dot{m}_{H e}}{U_{m} A}\left[1-\frac{\rho_{\text {air }}}{\rho_{H e}}\right]
\end{gathered}
$$

To find uncertainty of $\rho_{m i x}$, we perform Kline-McClintock Analysis on Equation B.3.

$$
w_{\rho_{\text {mix }}}=\left[\begin{array}{l}
\left(\frac{\partial \rho_{\text {mix }}}{\partial \rho_{\text {air }}} \times w_{\rho_{\text {air }}}\right)^{2}+\left(\frac{\partial \rho_{\text {mix }}}{\partial \dot{m}_{H e}} \times w_{\dot{m}_{H e}}\right)^{2}+\left(\frac{\partial \rho_{\text {mix }}}{\partial U_{m}} \times w_{U_{m}}\right)^{2} \\
+\left(\frac{\partial \rho_{\text {mix }}}{\partial A_{c}} \times w_{A_{c}}\right)^{2}+\left(\frac{\partial \rho_{m i x}}{\partial \rho_{H e}} \times w_{\rho_{\text {air }}}\right)^{2}
\end{array}\right]^{\frac{1}{2}}
$$

Individual uncertainties for the various parameters in Equation B.4a were determined. The temperature of the air and helium stream was measured using a E-type thermocouple. Since the uncertainty of the temperature measurement was $\pm 0.01^{\circ} \mathrm{C}$, the uncertainties of the densities of air and helium was determined from their respective equations of state (see Appendix C) by considering a deviation of $\pm 0.01^{\circ} \mathrm{C}$ from a 
temperature of $20{ }^{\circ} \mathrm{C}$. The uncertainties were evaluated as: $w_{\rho_{\text {arr }}}=0.00366 \mathrm{~kg} / \mathrm{m}^{3}$. and $w_{\rho_{H e}}=0.004583 \mathrm{~kg} / \mathrm{m}^{3}$. The uncertainty in velocity was evaluated as $w_{U_{m}}=0.0154 \mathrm{~m} / \mathrm{s}$ while the uncertainty in the cross sectional area was estimated as $w_{A}=0.0001 \mathrm{~m}^{2}$. The uncertainty in the mass flow rate of helium was obtained as $w_{\dot{m}_{H e}}=1.532 \times 10^{-4} \mathrm{~kg} / \mathrm{s}$.

$$
w_{\rho_{\text {mix }}}=\left[\begin{array}{l}
\left\{\left(1-\frac{\dot{m}_{H e}}{U_{m} A \rho_{H e}}\right) \times w_{\rho_{\text {air }}}\right\}^{2}+\left\{\frac{1}{U_{m} A}\left(1-\frac{\rho_{\text {air }}}{\rho_{H e}}\right) \times w_{\dot{m}_{H e}}\right\}^{2} \\
+\left\{\frac{-\dot{m}_{H e}}{U_{m}^{2} A}\left(1-\frac{\rho_{\text {air }}}{\rho_{H e}}\right) \times w_{U_{m}}\right\}^{2}+\left\{\frac{-\dot{m}_{H e}}{U_{m} A^{2}}\left(1-\frac{\rho_{\text {air }}}{\rho_{H e}}\right) \times w_{A}\right\}^{2} \\
+\left\{\left(\frac{\dot{m}_{H e}}{U_{m} A} \frac{\rho_{\text {air }}}{\rho_{H e}^{2}}\right) \times w_{\rho_{H e}}\right\}^{2}
\end{array}\right]^{\frac{1}{2}}
$$

Assuming densities at a temperature of $20{ }^{\circ} \mathrm{C}$, i.e. $\rho_{\text {air }}=1.21 \mathrm{~kg} / \mathrm{m}^{3}$ and $\rho_{\mathrm{He}}=0.16585$ $\mathrm{kg} / \mathrm{m}^{3}$, the uncertainty in Atwood Number of the flow was evaluated and was tabulated in Table B.1. 
TABLE B.1 Uncertainty in Atwood number for various experimental run conditions

\begin{tabular}{|c|c|c|c|c|c|}
\hline$A_{\mathrm{t}}$ & $U_{\mathrm{m}}(\mathrm{m} / \mathrm{s})$ & $\dot{m}_{H e}(\mathrm{~kg} / \mathrm{s})$ & $\rho_{\text {mix }}\left(\mathrm{kg} / \mathrm{m}^{3}\right)$ & $w_{\rho_{\text {mix }}}$ & $w_{A_{t}}$ \\
\hline 0.035 & 0.60 & 0.0030 & 1.1243 & 0.00929 & \pm 0.0024 \\
\hline 0.097 & 0.84 & 0.0106 & 0.9962 & 0.00662 & \pm 0.0036 \\
\hline 0.250 & 1.21 & 0.0345 & 0.7258 & 0.00509 & \pm 0.0036 \\
\hline 0.496 & 1.70 & 0.0805 & 0.4078 & 0.00435 & \pm 0.0042 \\
\hline 0.750 & 2.09 & 0.1287 & 0.1667 & 0.00409 & \pm 0.0053 \\
\hline
\end{tabular}




\section{B.4 Error in Visualization}

Snider and Andrews (1994) reported on the sources of fixed and random errors associated with their dye experiment. Fixed errors such as variations in background lighting were removed by employing the above correction during data analysis. Random errors though often uncontrollable were small in a well designed experiment. The volume fraction $f_{\mathrm{v}}$, was a random variable determined at each pixel location of an image. Since $f_{\mathrm{v}}$ was a line average through the mixing layer, it was also an estimate of the mean volume fraction, $\overline{f_{v}}$, which was obtained by averaging over all images. From the Central Limit Theorem, the variance $\overline{f_{v}}$ was given by

$$
\operatorname{Var}\left(\overline{\mathrm{f}_{\mathrm{v}}}\right)=\sigma_{\text {smoke }}^{2}=\frac{\mathrm{B}_{2}}{\mathrm{~N}}
$$

where $B_{2}$. was the normalized two-fluid density variance and $N$ was the total number of volume fraction measurements. The total number of volume fraction measurements was the product of the number of images acquired in a run (400 in the 0.035 Atwood imaging experiment) and the number of samples in the line average through the mix (361). Through the center of the mix, the line average gave a very large sample because of the relatively large net material flux through the centerline of the mix. The variance in the measured $\overline{f_{v}}$ close to the centerline was thus negligible (Wilson 2002). At the edge of the mixing layer where $\overline{f_{v}}$ was 0.05 or 0.95 , the ratio decreases when total number of image $(\sim 400)$, making the error about $\overline{f_{v}} \pm 0.025$. This was also evident from the 
wiggles observed in the fraction profiles at mixture fraction values of 0 and 1 , shown in Figure 6.3.

\section{B.5 Error in Hot-Wire Measurements}

Studies conducted by Pierce and Ezekwe (1976) and Ezekwe et al. (1978) reveal that errors involved in use of a multi-position single wire probe were comparable to an $\mathrm{X}$ - wire probe. There were two significant elements in the use of multi-position measurements of turbulent fluctuations. Firstly, the method was based on sequential measurement at different orientations and was sensitive to changes in flow condition. Care was taken to ensure that the mean flow remained constant and the probe was placed at the same location for each successive run. Secondly, since the mean flow field was predominately one-dimensional ( $V=W=0$ ), the technique of Bruun (1972) reduced to three measurements instead of the six roll positions required to resolve a three dimensional mean flow. This reduced the errors involved in the measurements as the number of positions was cut in half. A Kline McClintock uncertainty analysis was undertaken for the multi-position multi-overheat method. Swaminathan et al. (1986) carried out an analysis for parallel probe-stem orientation in a flow field similar to the one used here. They plotted the errors associated with the mean velocity as a function of the turbulence intensity of the flow. Their study shows that if the turbulence intensity of the flow varied between $10-12 \%$, the maximum error associated with the SN-wire measurements would be between $0.5-3.0 \%$, which was consistent with our observations. 


\section{APPENDIX C}

\section{PROPERTIES OF FLUIDS}

\section{C.1 Properties of Helium}

(a) Density :Polynomial fit for Equation of State for Helium ( $\rho$ in $\mathrm{kg} / \mathrm{m}^{3}$ and $T$ in ${ }^{\circ} \mathrm{C}$ ) for range of $0-50^{\circ} \mathrm{C}$ at 1 atm (McCarty 1973) :

$$
\rho_{H e}=1.804 \times 10^{-6} T^{2}-6.381320 \times 10^{-4} T+1.774853089 \times 10^{-1}
$$

(b) Viscosity: At $300 \mathrm{~K}$, absolute viscosity $(\mu)$ of helium was $20.1 \times 10^{-6} \mathrm{~kg} / \mathrm{m} \mathrm{s}$.

(c) Thermal conductivity: At $300 \mathrm{~K}$, thermal conductivity $(k)$ of Helium was 0.149 $\mathrm{W} / \mathrm{mK}$.

(d) Specific Heat: At 300K, specific heat at constant pressure $\left(C_{\mathrm{p}}\right)$ was $5200 \mathrm{~J} / \mathrm{kg} \mathrm{K}$.

\section{C.2 Properties of Air}

(a) Density: Polynomial fit for Equation of State for Air for range of $0-50{ }^{\circ} \mathrm{C}$ at 1 atm

$$
\rho_{\text {air }}=-2 \times 10^{-8} T^{4}+4 \times 10^{-8} T^{3}-2 \times 10^{-4} T^{2}-0.0037 T+1.2895
$$

(b) Viscosity: At $300 \mathrm{~K}$, absolute viscosity $(\mu)$ of Air $=18.43 \times 10^{-6} \mathrm{~kg} / \mathrm{m} \mathrm{s}$.

(c) Thermal conductivity: At $300 \mathrm{~K}$, thermal conductivity $(k)$ of Air $=0.0267 \mathrm{~W} / \mathrm{m} \mathrm{K}$.

(d) Specific Heat: At 300K, specific heat at constant pressure $\left(C_{\mathrm{p}}\right)$ was $1005 \mathrm{~J} / \mathrm{kg} \mathrm{K}$. 


\section{APPENDIX D}

\section{CALIBRATION CURVE FITS \& ANALYSIS SOFTWARE}

\section{D.1 Table-Curve 3D Fits for $E=f(U, \rho)$}

\section{Statistics for Wire \# $1 @$ Overheat Ratio 1.9}

\begin{tabular}{|c|c|c|c|c|c|c|c|}
\hline \multirow{2}{*}{\multicolumn{2}{|c|}{$\begin{array}{l}r^{2} \text { Coef Det } \\
0.9977113513\end{array}$}} & \multirow{2}{*}{\multicolumn{2}{|c|}{$\begin{array}{l}\text { DF Adj r² } \\
0.9974212409\end{array}$}} & Fit Std Err & \multicolumn{3}{|l|}{ F-value } \\
\hline & & & & 0.020107227 & 3923.4514176 & & \\
\hline \multirow{2}{*}{$\begin{array}{l}\text { Parm } \\
\text { a }\end{array}$} & \multicolumn{2}{|l|}{ Value } & Std Error & t-value & \multicolumn{2}{|c|}{ 95.00\% Confidence Limits } & $P>|t|$ \\
\hline & \multicolumn{2}{|c|}{1.597567133} & 0.011529454 & $4 \quad 138.5639871$ & 1.574583587 & 1.62055068 & 0.0000 \\
\hline $\mathrm{b}$ & \multicolumn{2}{|c|}{0.146337686} & 0.056832895 & $5 \quad 2.574876487$ & 0.03304338 & 0.259631991 & 0.01208 \\
\hline c & \multicolumn{2}{|c|}{1.336098575} & 0.254887787 & $7 \quad 5.241908967$ & 0.827989058 & 1.844208092 & 0.00000 \\
\hline $\mathrm{d}$ & \multicolumn{2}{|c|}{0.283005712} & 0.136791988 & 32.06887638 & 0.010315869 & 0.555695556 & 0.04215 \\
\hline $\mathrm{e}$ & \multicolumn{2}{|c|}{-0.34066712} & 0.040271311 & $1 \quad-8.45930035$ & -0.42094651 & -0.26038773 & 0.00000 \\
\hline$f$ & \multicolumn{2}{|c|}{-0.01302054} & 0.036464239 & $\begin{array}{l}9 \\
-0.35707696\end{array}$ & -0.08571067 & 0.059669593 & 0.72208 \\
\hline $\mathrm{g}$ & \multicolumn{2}{|c|}{0.010817117} & 0.003417177 & $7 \quad 3.165512336$ & 0.004005099 & 0.017629136 & 0.00227 \\
\hline $\mathrm{h}$ & \multicolumn{2}{|c|}{1.12825612} & 0.159137316 & 67.08 & 021679 & 1.445490561 & 0.00000 \\
\hline i & \multicolumn{2}{|c|}{0.562145551} & 0.059854749 & 9.391828791 & 0.44282729 & 0.681463811 & 0.00000 \\
\hline \multirow{2}{*}{\multicolumn{2}{|c|}{$\begin{array}{l}X \text { at Fn Zmin } \\
0.3956258391\end{array}$}} & \multicolumn{2}{|c|}{$\mathrm{Y}$ at $\mathrm{Fn} \mathrm{Zmin}$} & \multicolumn{4}{|l|}{ Fn Zmin } \\
\hline & & \multirow{2}{*}{\multicolumn{2}{|c|}{$\begin{array}{l}1.201 \\
Y \text { at Fn Zmax }\end{array}$}} & \multirow{2}{*}{\multicolumn{4}{|c|}{$\begin{array}{l}1.5609701 \\
\text { Fn Zmax }\end{array}$}} \\
\hline \multirow{2}{*}{\multicolumn{2}{|c|}{$\begin{array}{l}X \text { at Fn Zmax } \\
3.0192032989\end{array}$}} & & & & & & \\
\hline & & \multicolumn{2}{|c|}{0.16585} & .9855376207 & & & \\
\hline
\end{tabular}

Procedure

GaussElim

$\mathrm{r}^{2}$ Coef Det DF Adj $\mathrm{r}^{2} \quad$ Fit Std Err

$\begin{array}{lll}0.9977113513 & 0.9974212409 & 0.020107227\end{array}$

$\begin{array}{llllll}\text { Source } & \text { Sum of Squares } & \text { DF } & \text { Mean Square } & \text { F Statistic } & \text { P }>F \\ \text { Regr } & 12.690029 & 8 & 1.5862537 & 3923.45 & 0.00000 \\ \text { Error } & 0.029109642 & 72 & 0.00040430058 & & \\ \text { Total } & 12.719139 & 80 & & & \end{array}$

Description: WIre \# 1 Overheat Ratio = 1.9

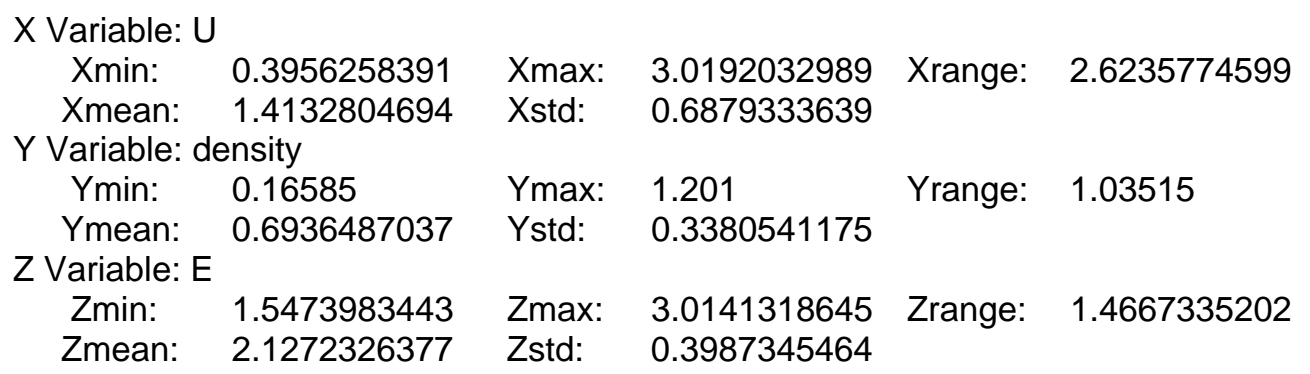




\section{Statistics for Wire \# $1 @$ Overheat Ratio 1.6}

\begin{tabular}{|c|c|c|c|c|c|c|}
\hline \multirow{2}{*}{\multicolumn{2}{|c|}{$\begin{array}{l}r^{2} \text { Coef Det } \\
0.9974645004\end{array}$}} & \multirow{2}{*}{$\begin{array}{l}\text { DF Adj r }{ }^{2} \\
0.9971385076\end{array}$} & Fit Std Err & \multirow{2}{*}{\multicolumn{3}{|c|}{$\begin{array}{l}\text { F-value } \\
3491.42131\end{array}$}} \\
\hline & & & 0.0181294158 & & & \\
\hline \multirow{2}{*}{$\begin{array}{l}\text { Parm } \\
\text { a }\end{array}$} & Value & Std Error & t-value & \multicolumn{2}{|c|}{ 95.00\% Confidence Limits } & \multirow{2}{*}{$\begin{array}{c}P>|t| \\
0.00000\end{array}$} \\
\hline & 1.372360607 & 0.010487826 & $6 \quad 130.8527212$ & 1.351448475 & 1.393272738 & \\
\hline $\mathrm{b}$ & 0.113413401 & 0.050924323 & $3 \quad 2.227096902$ & 0.011873186 & 0.214953617 & 0.02911 \\
\hline c & 1.151771106 & 0.225227138 & 85.113820284 & 0.702680948 & 1.600861264 & 0.0000 \\
\hline $\mathrm{d}$ & 0.265377675 & 0.115064094 & $4 \quad 2.306346541$ & 0.035946388 & 0.494808962 & 0.02401 \\
\hline $\mathrm{e}$ & -0.28411545 & 0.035787857 & $7-7.93887861$ & -0.35547441 & -0.21275649 & 0.0000 \\
\hline$f$ & -0.0225251 & 0.0383284 & -0.58768692 & -0.09894976 & 0.053899559 & 0.5586 \\
\hline $\mathrm{g}$ & 0.011794476 & 0.003714531 & $1 \quad 3.17522649$ & 0.004387912 & 0.019201039 & 0.0022 \\
\hline $\mathrm{h}$ & 1.116921313 & 0.163582617 & $7 \quad 6.827872858$ & 0.790746838 & 1.443095788 & 0.0000 \\
\hline $\mathrm{i}$ & 0.55947936 & 0.061337143 & 39.12137955 & 0.437176571 & 0.68178215 & 0.0000 \\
\hline
\end{tabular}

$\begin{array}{lll}X \text { at Fn Zmin } & Y \text { at Fn Zmin } & F n Z m i n \\ 0.3956258391 & 1.201 & 1.344550273 \\ X \text { at Fn Zmax } & Y \text { at Fn Zmax } & F n Z m a x \\ 2.8842507801 & 0.16585 & 2.5642614803\end{array}$

Procedure

GaussElim

$r^{2}$ Coef Det DF Adj $r^{2} \quad$ Fit Std Err

$0.9974645004 \quad 0.9971385076 \quad 0.0181294158$

Source Sum of Squares DF Mean Square F Statistic P>F

$\begin{array}{llllll}\text { Regr } & 9.1803632 & 8 & 1.1475454 & 3491.42 & 0.00000\end{array}$

$\begin{array}{llll}\text { Error } & 0.023335976 & 71 & 0.00032867572 \\ \text { Total } & 9.2036992 & 79 & \end{array}$

Description: Wire 1 Overheat Ratio $=1.6$

$X$ Variable: $U$

Xmin: $\quad 0.3956258391 \quad X \max : 2.8842507801$ Xrange: 2.4886249411

Xmean: 1.3958217673 Xstd: 0.67536498

$Y$ Variable: density

$\begin{array}{llll}\text { Ymin: } & 0.16585 & \text { Ymax: } 1.201 & \text { Yrange: } 1.03515\end{array}$

Ymean: 0.6873068125 Ystd: 0.3353030748

Z Variable: E

Zmin: $\quad 1.3339 \quad$ Zmax: 2.5920828496 Zrange: 1.2581828496

Zmean: $\quad 1.8282989817$ Zstd: 0.3413246571 
Statistics for Wire \# 2 @ Overheat Ratio 1.9

\begin{tabular}{|c|c|c|c|c|c|}
\hline $\begin{array}{ll}r^{2} \text {. Coef Det } & D \\
0.9949220988 & \end{array}$ & \multicolumn{2}{|c|}{$\begin{array}{cc}\text { DF Adj r } r^{2} & \text { Fit Std Err } \\
0.9942784212\end{array}$} & \\
\hline Parm Value & Std Erro & $\mathrm{t}$-value & \multicolumn{2}{|c|}{ 95.00\% Confidence Limits } & $P>|t|$ \\
\hline a 1.601146136 & 0.015896172 & 100.7252667 & 1.569457697 & 1.632834576 & 0.00000 \\
\hline b -0.03010811 & 0.079625043 & -0.37812366 & -0.18883773 & 21509 & 0.70645 \\
\hline c 0.709512864 & 0.390172234 & 1.818460673 & -0.06828127 & 1.487306998 & 0.07315 \\
\hline d 0.630059365 & 0.183635538 & 3.4310 & 0.26 & 0.99613012 & 0.00100 \\
\hline e -0.32691708 & 0.063336565 & -5.16158521 & -0.45317 & -0.20065794 & 0.00000 \\
\hline f -0.11250296 & 0.050668786 & -2.2203603 & -0.21350 & -0.01149658 & 0.02954 \\
\hline g 0.013982988 & 80.004823267 & 2.899070111 & 0.00436 & 0.023597995 & \\
\hline & & & & & \\
\hline & & & & & \\
\hline
\end{tabular}

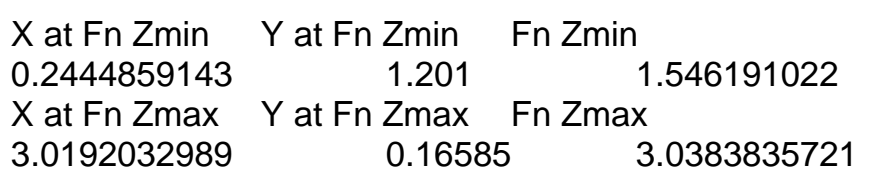

Procedure

GaussElim

$r^{2}$. Coef Det DF Adj r $r^{2} \quad$ Fit Std Err

$0.9949220988 \quad 0.9942784212 \quad 0.0304227637$

Source Sum of Squares DF Mean Square

$\begin{array}{llll}\text { Regr } & 13.056737 & 8 & 1.6320921\end{array}$

$\begin{array}{llll}\text { Error } & 0.066639208 & 72 & 0.00092554455\end{array}$

Total $13.123376 \quad 80$

Description: Wire 2 OR 1.9

X Variable: $U$

Xmin:

Xmean:

0.2444859143

1.4099503227

Xmax:

Xstd:

3.0192032989 Xrange:

2.7747173846

Y Variable: density

Ymin:

0.16585

0.6936487037

Ymax:

Ystd:

1.201

0.3380541175

Z Variable: $\mathrm{E}$

Zmin:

Zmean:

$1.535509596 \quad$ Zmax:

$2.1310283157 \quad Z s t d:$
3.0173099995

0.4050212364
Zrange:

1.4818004035 


\section{Statistics for Wire \# 2 @ Overheat Ratio 1.6}

\begin{tabular}{|c|c|c|c|c|c|}
\hline $\begin{array}{l}r^{2} \text { Coef Det } \\
0.9900189948\end{array}$ & DF Adj $r^{2}$ & $\begin{array}{l}\text { Fit Std Err } \\
537969\end{array}$ & \multicolumn{3}{|l|}{ F-value } \\
\hline Parm Value & Std Error & t-value & $95.00 \%$ Confic & ence Limits & $P>|t|$ \\
\hline a 1.351239598 & 80.019098402 & 70.75144762 & 1.313167629 & 1.389311567 & 0.00000 \\
\hline b -0.16850111 & 10.090209132 & -1.86789414 & -0.34832973 & 0.011327509 & 0.06585 \\
\hline c 0.166329933 & 30.436832679 & 0.380763486 & -0.7044801 & 1.037139963 & 0.70450 \\
\hline d 0.827636942 & 20.195697694 & 4.229160426 & 0.437520719 & 1.217753164 & 0.00007 \\
\hline e -0.25085822 & $2 \quad 0.071317698$ & -3.51747502 & -0.39302746 & -0.10868899 & 0.00076 \\
\hline$f \quad-0.22654812$ & 0.067813342 & -3.34076029 & -0.36173154 & -0.09136469 & 0.00133 \\
\hline g 0.022792657 & 70.006741552 & 3.380921159 & 0.009353618 & 0.036231696 & 0.00117 \\
\hline h 0.371168077 & $7 \quad 0.308308796$ & 1.203884162 & -0.24343428 & 0.98577043 & 0.23258 \\
\hline i 0.602162332 & 0.128871363 & 4.672584478 & 0.345261964 & 0.8590627 & 0.00001 \\
\hline
\end{tabular}

\begin{tabular}{|c|c|c|}
\hline$X$ at Fn Zmin & $\mathrm{Y}$ at $\mathrm{Fn} \mathrm{Zmin}$ & Fn Zmin \\
\hline 0.2444859143 & 1.201 & 1.3215901449 \\
\hline $\mathrm{X}$ at Fn Zmax & $Y$ at Fn Zmax & Fn Zmax \\
\hline 3.0192032989 & 0.1658 & 2.5935416098 \\
\hline
\end{tabular}

Procedure

GaussElim

$r^{2}$. Coef Det DF Adj r $r^{2}$ Fit Std Err

$0.9900189948 \quad 0.9887537969 \quad 0.0362575999$

Source Sum of Squares DF Mean Square

$\begin{array}{llll}\text { Regr } & 9.3885786 & 8 & 1.1735723\end{array}$

$\begin{array}{llll}\text { Error } & 0.094652176 & 72 & 0.0013146135\end{array}$

Total $9.4832307 \quad 80$

Description: Wire 2 OR 1.6

X Variable: $U$

Xmin:

Xmean:

0.2444859143

1.4083486408

Xmax:

Xstd:

3.0192032989

0.6920767533

$Y$ Variable: density

Ymin:

Ymin:

0.16585

0.6936487037

Ymax:

Ystd:

1.201

0.3380541175

Z Variable: $\mathrm{E}$

Zmin:

Zmean:

1.3107898392

1.8144332379

Zmax:

Zstd:
2.5703616892 Zrange:

0.3442969419
$892.713 \quad 0.00000$

2.7747173846

Yrange: $\quad 1.03515$

1.25957185 
Statistics for Wire \# 3 @ Overheat Ratio 1.9

\begin{tabular}{|c|c|c|c|c|c|}
\hline $\begin{array}{l}r^{2} \text { Coef Det } \\
0.9990933242\end{array}$ & \multicolumn{2}{|c|}{$\begin{array}{cc}\text { DF Adj r }^{2}, \quad \text { Fit Std Err } \\
0.9989783934\end{array}$} & \multicolumn{3}{|l|}{ F-value } \\
\hline Parm Value & Std Error & t-value & \multicolumn{2}{|c|}{ 95.00\% Confidence Limits } & $P>|t|$ \\
\hline a 1.665437866 & 60.007233693 & 230.2334069 & 1.651017762 & 1.67985797 & 0.00000 \\
\hline b -0.00973969 & 0.044769157 & -0.21755353 & -0.09898537 & 0.079505995 & 0.82839 \\
\hline c 0.607496352 & 20.188352893 & 3.225309381 & 0.232021723 & 0.982970982 & 0.00189 \\
\hline d 0.608484511 & 10.101319832 & 6.005581495 & 0.406507116 & 0.810461905 & 0.00000 \\
\hline e -0.31246583 & 0.027109153 & -11.5262115 & -0.36650694 & -0.25842473 & 0.00000 \\
\hline f -0.11734193 & 0.026803272 & -4.37789564 & -0.17077328 & -0.06391058 & 0.00004 \\
\hline g 0.017656372 & 20.002243834 & 7.868840976 & 0.013183371 & 0.022129374 & 0.00000 \\
\hline h 0.65093585 & 0.112824733 & 5.76944286 & 0.426023855 & 0.875847845 & 0.00000 \\
\hline i 0.5325526 & 0.037851832 & 14.06940105 & 0.457096352 & 0.608008847 & 0.00000 \\
\hline
\end{tabular}

$\mathrm{X}$ at Fn Zmin $\quad \mathrm{Y}$ at Fn Zmin Fn Zmin

$0.2444859143 \quad 1.201 \quad 1.6159437137$

$\mathrm{X}$ at Fn Zmax $\mathrm{Y}$ at Fn Zmax Fn Zmax

$2.9517522199 \quad 0.16585 \quad 3.1845791151$

Procedure

GaussElim

$r^{2}$. Coef Det DF Adj $r^{2} . \quad$ Fit Std Err

$\begin{array}{lll}0.9990933242 & 0.9989783934 & 0.0133819831\end{array}$

\begin{tabular}{llllll} 
Source & \multicolumn{2}{c}{ Sum of Squares } & DF Mean Square & \multicolumn{2}{c}{ F Statistic P P F } \\
Regr & 14.207821 & 8 & 1.7759776 & 9917.37 & 0.00000
\end{tabular}

$\begin{array}{llll}\text { Regr } & 14.207821 & 8 & 1.7759776 \\ \text { Error } & 0.012893578 & 72 & 0.00017907747\end{array}$

Total $14.220714 \quad 80$

Description: Wire 3 OR 1.9

$X$ Variable: $U$

Xmin: $\quad 0.2444859143 \quad$ Xmax: 2.9517522199 Xrange: 2.7072663055

Xmean: $\quad 1.4091175933 \quad$ Xstd: $\quad 0.690620275$

Y Variable: density

$\begin{array}{clllll}\text { Ymin: } & 0.16585 & \text { Ymax: } & 1.201 & \text { Yrange: } & 1.03515 \\ \text { Ymean: } & 0.6936487037 & \text { Ystd: } & 0.3380541175 & & \\ & & & & & \\ \text { Variable: E } & & & & & \\ \text { Zmin: } & 1.5829157049 & \text { Zmax: } & 3.1817187346 & \text { Zrange: } & 1.5988030297 \\ \text { Zmean: } & 2.2430352037 & \text { Zstd: } & 0.4216146701 & & \end{array}$




\section{Statistics for Wire \# 3 @ Overheat Ratio 1.6}

\begin{tabular}{|c|c|c|c|c|c|}
\hline \multirow{3}{*}{$\begin{array}{l}r^{2} \text { Coef Det } \\
0.9992785047 \\
\text { Parm Value }\end{array}$} & \multirow{3}{*}{$\begin{array}{l}\text { DF Adj } r^{2} \\
0.9991870476 \\
\text { Std Error }\end{array}$} & \multirow{2}{*}{$\begin{array}{l}\text { Fit Std Err } \\
0.010102873\end{array}$} & \multicolumn{2}{|l|}{ F-value } & \multirow[b]{3}{*}{$P>|t|$} \\
\hline & & & $39 \quad 1246$ & 094188 & \\
\hline & & t-value & \multicolumn{2}{|c|}{ 95.00\% Confidence Limits } & \\
\hline a 1.441007623 & 0.00543125265 & 3178563 & 1.430180624 & 1.451834622 & 0.00000 \\
\hline b 0.040619202 & 0.0321942331 .2 & 61691866 & -0.02355883 & 0.104797234 & 0.21113 \\
\hline c 0.720049688 & 0.136803665 .2 & 63380298 & 0.447336576 & 0.9927628 & 0.00000 \\
\hline d 0.360066215 & 0.0663528765. & 426535162 & 0.227794174 & 0.492338256 & 0.00000 \\
\hline e -0.27152962 & $0.019923557-1$ & 3.6285714 & -0.31124651 & -0.23181274 & 0.00000 \\
\hline f -0.08085121 & $0.022436109-3$. & 60361991 & -0.12557678 & -0.03612564 & 0.00057 \\
\hline g 0.015140754 & $0.00192054 \quad 7.8$ & 383593717 & 0.011312228 & 0.018969279 & 0.00000 \\
\hline h 0.790022957 & 0.0944621478 .3 & 363381354 & 0.601716109 & 0.978329806 & 0.00000 \\
\hline i 0.503828797 & 0.0326764915 & .41869369 & 0.438689404 & 0.56896819 & 0.00000 \\
\hline
\end{tabular}

$\begin{array}{lll}X \text { at Fn Zmin } & Y \text { at Fn Zmin } & F n \text { Zmin } \\ 0.2444859143 & 1.201 & 1.3942238457 \\ X \text { at Fn Zmax } & Y \text { at Fn Zmax } & F n Z m a x \\ 3.0192032989 & 0.16585 & 2.7315137502\end{array}$

Procedure

GaussElim

$r^{2}$ Coef Det

0.9992785047

Source

Regr

Error

Total
DF Adj r ${ }^{2}$. 0.9991870476

Sum of Squares

10.178304

0.00000

0.0073489003

10.185653
Fit Std Err

0.0101028739

8

DF Mean Square

1.272288

$72 \quad 0.00010206806$

Description: Wire 3 OR 1.6

X Variable: $U$

Xmin:

Xmean:

0.2444859143

1.4083486408

$Y$ Variable: density

Ymin:

Ymean:

0.16585

0.6936487037

Z Variable: E

Zmin:

Zmean:

1.3858086175

1.9250929243
F Statistic $\quad P>F$ 12465.1

80
Ystd:

1.201

0.3380541175

Xmax:

3.0192032989

0.6920767533

Xrange:

2.7747173846

Yrange:

1.03515

Zmax:

2.7318

0.3568202064
Zrange:

1.3459913825 


\section{D.2 Table-Curve 3D Fits for $E=f(U, \theta)$}

\section{Statistics for Wire \# $1 @$ Overheat Ratio 1.9}

\begin{tabular}{|c|c|c|c|c|c|}
\hline$r^{2}$ Coef Det & DF Adj $r^{2}$ & Fit Std Err & \multicolumn{3}{|l|}{ F-value } \\
\hline 0.9995147458 & 0.9993529944 & 0.001958329 & \multicolumn{2}{|c|}{7003.2367144} & \\
\hline Parm Value & Std Error & t-value & \multicolumn{2}{|c|}{ 95.00\% Confidence Limits } & $P>|t|$ \\
\hline a 1.475824144 & 0.007869679 & 187.5329617 & 1.459831032 & 1.491817255 & 0.00000 \\
\hline b -0.28427433 & 0.06048153 & -4.70018423 & -0.40718759 & -0.16136108 & 0.00004 \\
\hline c -0.37247819 & 0.092262774 & -4.03714491 & -0.5599787 & -0.18497767 & 0.00029 \\
\hline$d-0.12640027$ & 0.090178225 & -1.40167177 & -0.30966447 & 0.05686393 & 0.17008 \\
\hline e -0.00053468 & 0.157045607 & -0.00340465 & -0.31968976 & 0.318620387 & 0.99730 \\
\hline f 0.039219844 & 0.016999352 & 2.307137555 & 0.004673004 & 0.073766685 & 0.02727 \\
\hline g 0.056463086 & 0.02521095 & 2.239625427 & 0.00522827 & 0.107697901 & 0.03176 \\
\hline h 0.036278739 & 0.028317399 & 1.281146556 & -0.02126914 & 26618 & 0.20881 \\
\hline i $\quad-0.00605342$ & 0.049086069 & -0.12332261 & -0.10580832 & 0.093701472 & 0.90258 \\
\hline j 0.004898091 & 0.012028149 & 0.407219057 & -0.01954605 & 0.02934223 & 0.68640 \\
\hline k 0.006813322 & 0.018458106 & 0.369123588 & -0.03069806 & 0.044324707 & 0.71432 \\
\hline
\end{tabular}

$\begin{array}{lll}X \text { at Fn Zmin } & Y \text { at Fn Zmin } & F n Z m i n \\ 0.3956258391 & 0.5235987756 & 1.5052091602 \\ X \text { at Fn Zmax } & Y \text { at Fn Zmax } & \text { Fn Zmax } \\ 3.0192032989 & 1.574231823 & 1.7832775762\end{array}$

Procedure

GaussElim

$r^{2}$. Coef Det 0.9995147458

Source

Regr

Error

Total
DF Adj $\mathrm{r}^{2}$

0.9993529944

Sum of Squares

0.26857786

0.00000

0.00013039181

0.26870825
44
Fit Std Err

0.0019583292

DF Mean Square

10

0.026857786

$34 \quad 3.8350533 e-06$

Description: OR=1.9

X Variable: $U$

Xmin:

Xmean:

0.3956258391

1.6816058445

Xmax:

Xstd:

3.0192032989

0.9437646045

Y Variable: angle

Ymin:

0.5235987756

Ymean:

1.5707963268

Ymax:

Ystd:

2.617993878

0.6836013564

Z Variable: $\mathrm{E}$

Zmin:

Zmean:
1.505531525

1.6470391601
Zmax:

Zstd:
1.7837676317 Zrange:

0.0781473335
F Statistic $\quad$ P $>F$ 7003.24

Xrange:

2.6235774599

Yrange:

2.0943951024

0.2782361067 


\section{Statistics for Wire \# 1 @ Overheat Ratio 1.6}

\begin{tabular}{|c|c|c|c|c|c|}
\hline$r^{2}$ Coef Det & DF Adj $r^{2}$ & & \multicolumn{3}{|l|}{ F-value } \\
\hline 0.9996491116 & 0.9995321488 & 0.001470203 & \multicolumn{2}{|c|}{9686.2915203} & \\
\hline Parm & Std Error & t-value & \multicolumn{2}{|c|}{ 95.00\% Confidence Limits } & \\
\hline a 1.25511155 & 0.006280124199 & 9.8545671 & 1.242348801 & 1.267874298 & 0.00000 \\
\hline b -0.33243229 & $0.050064093-6.6$ & 64013399 & -0.43417477 & -0.23068981 & 0.00000 \\
\hline c -0.37790052 & $\begin{array}{lll}0.06537744 & -5.7\end{array}$ & 8028942 & -0.51076347 & -0.24503758 & 0.00000 \\
\hline d 0.015406377 & 0.0797924680 .1 & 193080589 & -0.14675143 & 0.177564182 & 0.84804 \\
\hline e 0.219580206 & $0.12009047 \quad 1.8$ & 2845655 & -0.02447299 & 0.463633404 & 0.07627 \\
\hline f 0.037236431 & 0.0138196852 .6 & 694448705 & 0.009151453 & 0.065321409 & 0.01087 \\
\hline g 0.041637642 & 0.0176925872 .3 & 353394809 & 0.00568198 & 0.077593305 & 0.02453 \\
\hline h -0.00819299 & $0.024889249-0$ & 32917807 & -0.05877403 & 0.042388045 & 0.74404 \\
\hline i -0.07434363 & $0.037336234-1$ & 99119252 & -0.15021998 & 0.001532727 & 0.05454 \\
\hline j 0.00385509 & 0.0098431440 .3 & 391652329 & -0.01614858 & 0.023858765 & 0.69776 \\
\hline k 0.004537461 & 0.0129874970 .3 & 349371483 & -0.02185631 & 0.03093123 & 0.72897 \\
\hline
\end{tabular}

$\begin{array}{lll}X \text { at Fn Zmin } & Y \text { at Fn Zmin } & F n \text { Zmin } \\ 0.3956258391 & 0.5235987756 & 1.2897549055 \\ X \text { at Fn Zmax } & Y \text { at Fn Zmax } & F n \text { max } \\ 3.0192032989 & 1.5737276419 & 1.5355980971\end{array}$

Procedure

GaussElim

$r^{2}$. Coef Det

0.9996491116

Source

Regr

Error

Total
DF Adj $r^{2}$. 0.9995321488

Sum of Squares

0.20936891

0.00000

7.3490901e-05

0.2094424
Fit Std Err 0.0014702031

10

DF Mean Square

0.020936891

$34 \quad 2.1614971 \mathrm{e}-06$

Description: OR=1.6

$X$ Variable: $\mathrm{OR}=1.6$

Xmin:

Xmean:

0.3956258391

1.678577174

Y Variable: angle

Ymin:

Ymean:

0.5235987756

1.5707963268

Z Variable: $\mathrm{E}$

Zmin:

Zmean:
1.2891456302

1.4173058002
Xmax:

Xstd:

3.0192032989

0.9417026013

Ymax:

Ystd:

2.617993878

0.6836013564

Zmax:

Zstd:
1.5345410943 Zrange:

0.0689931484
F Statistic $\quad P>F$ 9686.29
Xrange:

2.6235774599

Yrange:

2.0943951024 


\section{Statistics for Wire \# $2 @$ Overheat Ratio 1.9}

\begin{tabular}{|c|c|c|c|c|c|}
\hline$r^{2}$ Coef Det & DF Adj $r^{2}$ & & \multicolumn{3}{|l|}{ F-value } \\
\hline 0.9993415652 & 0.9991220869 & 0.002450335 & \multicolumn{2}{|c|}{5160.3609714} & \\
\hline Parm Value & Std Error & t-value & \multicolumn{2}{|c|}{ 95.00\% Confidence Limits } & \\
\hline a 1.528483495 & 0.010434573 & 146.4826052 & 1.507277891 & 1.5496891 & 0.00000 \\
\hline b -0.14127707 & 0.074897179 & -1.88628019 & -0.29348645 & 0.010932316 & 0.06782 \\
\hline c -0.14263224 & 0.116625578 & 1.22299282 & -0.37964393 & 0.094379445 & 0.22974 \\
\hline$d-0.0859427$ & 0.124695202 & -0.68922222 & -0.33935384 & 0.167468435 & 0.49536 \\
\hline e 0.052526736 & 0.222556423 & 0.236015368 & -0.39976233 & 0.504815804 & 0.81484 \\
\hline f -0.06697387 & 0.01648477 & -4.06277278 & -0.10047496 & -0.03347279 & 0.00027 \\
\hline g -0.11115456 & 0.026369696 & -4.21523868 & -0.16474423 & -0.05756489 & 0.00017 \\
\hline h 0.020536696 & 0.03918836 & 0.524050928 & -0.05910363 & 0.100177026 & 0.60364 \\
\hline i -0.02778837 & 0.069735283 & -0.3984837 & -0.16950752 & 0.113930773 & 0.69277 \\
\hline j 0.005595253 & 0.012202244 & 0.458 & -0.01920269 & 93196 & 0.64948 \\
\hline k 0.007108192 & 0.019270013 & 0.368873251 & -0.03205319 & 0.046269571 & 0.71451 \\
\hline
\end{tabular}

$\begin{array}{lll}X \text { at Fn Zmin } & Y \text { at Fn Zmin } & F n Z m i n \\ 0.3956258391 & 0.5235987756 & 1.5335538162 \\ X \text { at Fn Zmax } & Y \text { at Fn Zmax } & F n Z m a x \\ 3.0192032989 & 1.578799829 & 1.83482394\end{array}$

Procedure

GaussElim

$r^{2}$ Coef Det 0.9993415652

Source

Regr

Error

Total
DF Adj $r^{2}$ 0.9991220869 Sum of Squares 0.30983552 0.00000 0.0002041409 0.31003966 44
Fit Std Err 0.0024503355 10 DF Mean Square 0.030983552 $34 \quad 6.0041442 \mathrm{e}-06$

Description: OR=1.9

$X$ Variable: $U$ Xmin: Xmean: 5160.36

Xstd: 0.9437646045

2.6235774599

Y Variable: angle

Ymin:

Ymean:

0.5235987756 1.5707963268

Z Variable: $\mathrm{E}$ Zmin: Zmean:
Ymax:

Ystd:

2.617993878 0.6836013564

Yrange:

2.0943951024

Zmax: Zstd:
Zrange:

0.3046015505 


\section{Statistics for Wire \# 2 @ Overheat Ratio 1.6}

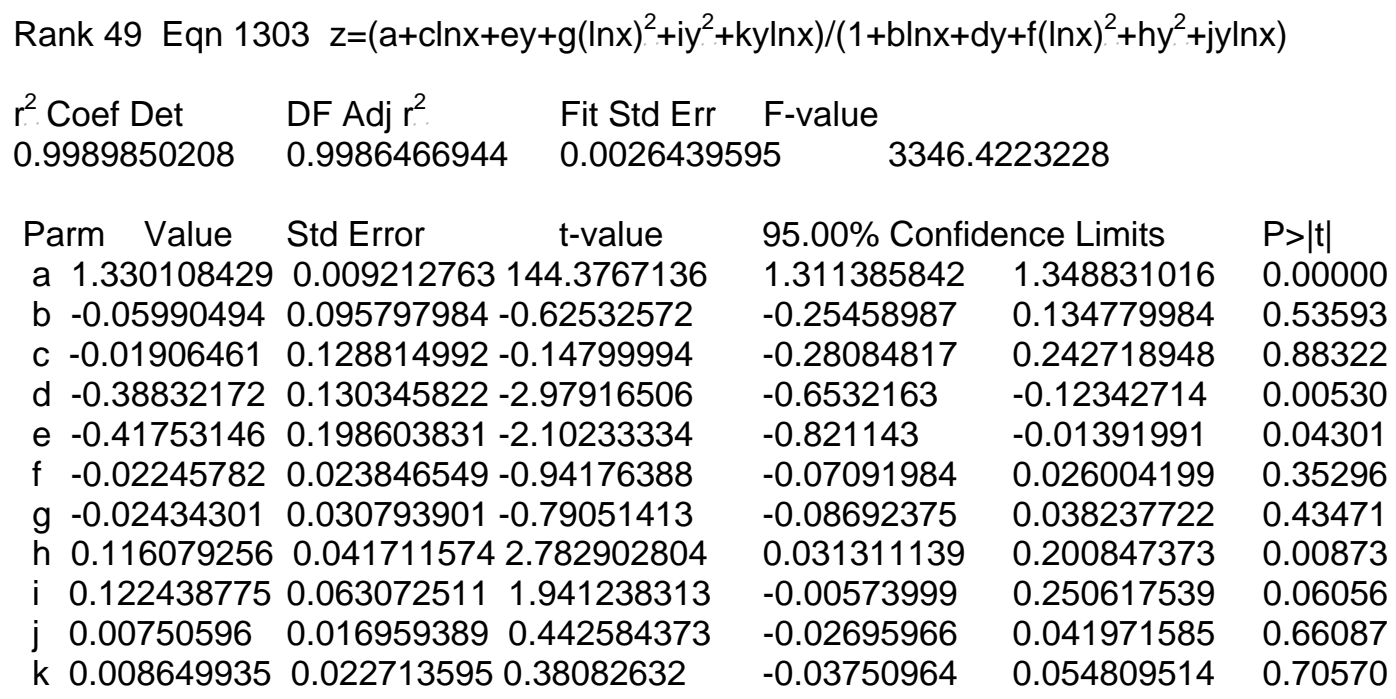

$\begin{array}{lll}X \text { at Fn Zmin } & Y \text { at Fn Zmin } & F n \text { Zmin } \\ 0.3956258391 & 0.5235987756 & 1.3211158231 \\ X \text { at Fn Zmax } & Y \text { at Fn Zmax } & F n \text { max } \\ 3.0192032989 & 1.5783331436 & 1.5785542988\end{array}$

Procedure

GaussElim

$r^{2}$. Coef Det 0.9989850208

Source

Regr

Error

Total
DF Adj $r^{2}$ 0.9986466944

Sum of Squares

0.23393239

0.00000

0.00023767775

0.23417007
Fit Std Err 0.0026439595

10

DF Mean Square

0.023393239

$34 \quad 6.990522 \mathrm{e}-06$

Description: OR=1.6

$X$ Variable: $\mathrm{OR}=1.6$

Xmin:

Xmean:

0.3956258391

1.678577174

Y Variable: angle

Ymin:

Ymean:

0.5235987756

1.5707963268

Z Variable: $\mathrm{E}$

Zmin:

Zmean:
1.3218501671

1.4540936143
Xmax:

Xstd:

3.0192032989

0.9417026013

Ymax:

Ystd:

2.617993878

0.6836013564

Zmax:

Zstd:
1.5761928844 Zrange:

0.0729523607
F Statistic $\quad P>F$ 3346.42
Xrange:

2.6235774599

Yrange:

2.0943951024

0.2543427173 


\section{Statistics for Wire \# 3 @ Overheat Ratio 1.9}

\begin{tabular}{|c|c|c|c|c|c|}
\hline \multirow{2}{*}{$\begin{array}{l}r^{2} \text {. Coef Det } \\
0.9992643285\end{array}$} & \multirow{3}{*}{$\begin{array}{l}\text { DF Adj r. } \\
0.9990191047 \\
\text { Std Error }\end{array}$} & \multirow{2}{*}{$\begin{array}{l}\text { Fit Std Err } \\
0.002797393\end{array}$} & \multicolumn{3}{|l|}{ F-value } \\
\hline & & & 4618. & 281637 & \\
\hline Parm Value & & t-value & \multicolumn{2}{|c|}{ 95.00\% Confidence Limits } & $P>|t|$ \\
\hline a 1.640444942 & 0.008846586 & 185.4325349 & 1.622466515 & 1.658423368 & 0.00000 \\
\hline b -0.13167946 & 0.117357619 & -1.12203587 & -0.37017883 & 0.106819919 & 0.26971 \\
\hline c -0.13812333 & 0.19397104 & -0.71208223 & -0.53231991 & 073251 & 0.48127 \\
\hline d -0.30652706 & 0.132241993 & -2.31792532 & -0.57527513 & -0.037779 & 0.02660 \\
\hline e -0.39052464 & 0.242242278 & -1.6121242 & -0.88282018 & 0.101770901 & 0.11618 \\
\hline f -0.01162024 & 0.024741767 & -0.469661 & -0.06190156 & 0.038661078 & 0.64160 \\
\hline g $\quad-0.01917814$ & 0.035851743 & -0.534929 & -0.09203765 & 0.053681371 & 0.59618 \\
\hline h 0.088685452 & 0.042001174 & 2.111499359 & 0.003328797 & 0.174042107 & 0.04216 \\
\hline i 0.109160966 & 0.076515917 & 1.426643892 & -0.04633809 & 0.264660019 & 0.16280 \\
\hline j 0.007867062 & 0.014500513 & 0.542536789 & -0.02160153 & 0.03733565 & 0.59099 \\
\hline k 0.010679033 & 0.023533102 & 0.453787724 & -0.03714598 & 0.05850405 & 0.65287 \\
\hline
\end{tabular}

$\begin{array}{lll}X \text { at Fn Zmin } & Y \text { at Fn Zmin } & F n Z m i n \\ 0.3956258391 & 0.5235987756 & 1.617408498 \\ X \text { at Fn Zmax } & Y \text { at Fn Zmax } & F n Z \max \\ 3.0866135159 & 1.5806148581 & 1.9303822406\end{array}$

Procedure

GaussElim

$r^{2}$. Coef Det

DF Adj $r^{2}$ 0.9990191047

0.9992643285

Source

Regr

Error

Total
Sum of Squares

0.36139525

0.00000

0.00026606391

0.36166131
Fit Std Err 0.0027973933

Description: OR=1.9

X Variable: $U$ Xmin: Xmean:

0.3956258391 1.6860998589

Y Variable: angle

Ymin:

Ymean:

0.5235987756

1.5707963268

Z Variable: E

Zmin:

Zmean:

1.6180739104

1.7785432644
F Statistic $\quad P>F$ 4618.23
Xmax:

Xstd:

3.0866135159

0.9504085444

Ymax:

Ystd:

2.617993878

0.6836013564

Yrange:

Xrange:

2.6909876768

Zmax:

Zstd:
1.9251673006

0.0906618734
Zrange:

0.3070933903 


\section{Statistics for Wire \# 3 @ Overheat Ratio 1.6}

\begin{tabular}{|c|c|c|c|c|c|}
\hline \multirow{3}{*}{$\begin{array}{l}r^{2} \text { Coef Det } \\
0.9982473332 \\
\text { Parm Value }\end{array}$} & \multirow{3}{*}{$\begin{array}{l}\text { DF Adj r }{ }^{2} \\
0.9978578517 \\
\text { Std Error }\end{array}$} & \multirow{2}{*}{$\begin{array}{l}\text { Fit Std Err } \\
0.003600960\end{array}$} & \multicolumn{3}{|l|}{ F-value } \\
\hline & & & \multicolumn{2}{|c|}{3010.5267393} & \multirow[b]{2}{*}{$P>|t|$} \\
\hline & & t-value & \multicolumn{2}{|c|}{ 95.00\% Confidence Limits } & \\
\hline a 1.37392523 & 0.007185574 & 191.2060415 & 1.359365876 & 1.388484589 & 0.00000 \\
\hline b $\quad-0.34380437$ & 0.045939344 & -7.4838763 & -0.43688632 & -0.25072241 & 0.00000 \\
\hline c -0.0140993 & 0.003476997 & -4.05502242 & -0.02114437 & -0.00705424 & 0.00025 \\
\hline d 0.155244715 & 50.022539909 & 6.887548531 & 0.109574522 & 0.200914908 & 0.00000 \\
\hline e -0.05496849 & 0.011582177 & -4.74595518 & -0.07843621 & -0.03150077 & 0.00003 \\
\hline f 0.000548518 & 0.00248603 & 0.220640294 & -0.00448866 & 0.005585694 & 0.82659 \\
\hline g -0.29561805 & 0.030917987 & -9.56136168 & -0.35826384 & -0.23297226 & 0.00000 \\
\hline h -0.00863752 & 0.011466478 & -0.75328406 & -0.03187081 & 0.014595777 & 0.45605 \\
\hline
\end{tabular}

$\begin{array}{lll}X \text { at Fn Zmin } & Y \text { at Fn Zmin } & F n Z m i n \\ 0.3956258391 & 0.5235987756 & 1.3759464962 \\ X \text { at Fn Zmax } & Y \text { at Fn Zmax } & F n Z \max \\ 3.0192032989 & 1.5789017539 & 1.6477800847\end{array}$

Procedure GaussElim $r^{2}$ Coef Det 0.9982473332

Source

Regr

Error

Total

\begin{tabular}{llcl} 
DF Adj r. & \multicolumn{3}{l}{ Fit Std Err } \\
0.9978578517 & 0.0036009605 \\
Sum of Squares & \multicolumn{2}{c}{ DF } & Mean Square \\
0.27326075 & 7 & & 0.03903725 \\
0.00000 & & & \\
0.00047977592 & & 37 & $1.2966917 e-05$ \\
0.27374053 & 44 & &
\end{tabular}

DF Adj $r^{2}$

Description: OR=1.6

$X$ Variable: $\mathrm{OR}=1.6$

$\begin{array}{llllll}\text { Xmin: } & 0.3956258391 & \text { Xmax: } & 3.0192032989 & \text { Xrange: } & 2.6235774599 \\ \text { Xmean: } & 1.678577174 & \text { Xstd: } & 0.9417026013 & & \end{array}$

Y Variable: angle Ymin: $\quad 0.5$

Ymean:

0.5235987756

Ymax:

2.617993878 Yrange:

2.0943951024

Z Variable: $\mathrm{E}$

Zmin:

1.5707963268

Ystd:

0.6836013564

F Statistic $\quad P>F$ 3010.53

Zmean:

1.3786410125

Zmax:

1.6480592055

Zrange:

0.269418193 


\section{D.3 MATLAB Routine to Solve for Density-Velocity Correlations}

$\%$ Script to solve for density, velocity and density-velocity

$\%$ correlations using Multi-position Multi-overheat technique

$\%$ Written by Arindam Banerjee - 04/01/2006

$\%$ See section 4.2 .3 for formulation of equations.

$\%$ Use 2 overheats. Overheat ratios used $\mathrm{OR}=1.9$ and $\mathrm{OR}=1.6$

$\%$ Use 3 directions. Directions used : 0, 30 and 45 degrees

$\%$ Fit Polynomial to calibration data using Table-Curve 3D

$\%$ Use E1 and E4 average voltage to solve for rho and U using TableCurve 3D

$\%$ software (In Table Curve 3D do the following Steps:

$\%$ a. Fit surface to data, b. Choose "review Surface Fit",

$\%$ c. Select "Quick Eval" to solve simultaneously for U and rho)

$\%$ Input rho and $\mathrm{U}$ (mean quantities)

$\mathrm{U}=0.5 ; \quad \%$ Velocity of flow in $\mathrm{m} / \mathrm{s}$

rho $=1.14858 \%$ Density of flow in $\mathrm{kg} / \mathrm{m}^{\wedge} 3$

$\%$ Input average voltage from each wire (6 measurements)

E1_avg $=1.5418 ; \%$ Avg Voltage for Position 1: 0 degrees; OR $=1.9$

E2 avg $=1.5642 ; \%$ Avg Voltage for Position $2: 30$ degrees; $O R=1.9$

E3 avg $=1.6184 ; \%$ Avg Voltage for Position $3: 45$ degrees; $O R=1.9$

E4_avg $=1.3178 ; \%$ Avg Voltage for Position 1: 0 degrees; $\mathrm{OR}=1.6$

E5 avg $=1.3292 ; \%$ Avg Voltage for Position $2: 30$ degrees; $O R=1.6$

E6_avg $=1.3826 ; \%$ Avg Voltage for Position $3: 45$ degrees; $O R=1.6$

$\%$ Input rms voltage from each wire (6 measurements)

E1_rms $=0.0243 ; \%$ RMS Voltage for Position 1: 0 degrees; OR $=1.9$

E2_rms $=0.0282 ; \%$ RMS Voltage for Position $2: 30$ degrees; OR $=1.9$

E3_rms $=0.0331 ; \%$ RMS Voltage for Position $3: 45$ degrees; OR $=1.9$

E4_rms $=0.0202 ; \%$ RMS Voltage for Position 1: 0 degrees; $O R=1.6$

E5_rms $=0.0243 ; \%$ RMS Voltage for Position $2: 30$ degrees; OR $=1.6$

E6_rms $=0.0281 ; \%$ RMS Voltage for Position $3: 45$ degrees; OR $=1.6$

$\%$ Obtain values of $\mathrm{dE} / \mathrm{dU}, \mathrm{dE} / \mathrm{drho}$ and $\mathrm{dE} / \mathrm{dtheta}$ from Table curve 3D.

$\%$ Use the following Steps

$\%$ a. Fit surface to data, b. Choose "review Surface Fit",

$\%$ c. From drop-down menu, select proper derivative

$\%$ d. Select "Quick Eval" to evaluate derivative at given U and rho

$\%$ (mean quantities)

$\%$ Input wire sensitivities (derivatives)

\%Wire \#1@ Overheat Ratio 1.9

de_du_w1o1=0.128826176;de_dr_w1o1=-0.492858699;de_dt_w1o1=0.00253;

\%Wire \#2@ Overheat Ratio 1.9

de_du_w2o1=0.120273648;de_dr_w2o1=-0.465683243; de_dt_w2o1=0.06648;

\%Wire \#3@ Overheat Ratio 1.9

de_du_w3o1=0.15059834;de_dr_w3o1=-0.484261853;de_dt_w3o1 $=0.06984$;

\%Wire \#1@ Overheat Ratio 1.6

de_du_w1o2 $=0.110776362 ;$ de_dr_w1o2 $=-0.407820658 ; d e \_d t \_w 102=0.00093$; 
\%Wire \#2@ Overheat Ratio 1.6

de_du_w2o2=0.114994238;de_dr_w2o2=-0.322425332;de_dt_w2o2=0.05382;

\%Wire \#3@ Overheat Ratio 1.6

de_du_w3o2 $=0.125414491 ;$ de_dr_w3o2 $=-0.43574187 ;$ de_dt_w3o2 $=0.06855$;

$\%$ Evaluate coefficients of A matrix (Refer to Equation $4.25 \mathrm{a}-\mathrm{f}$ )

$\mathrm{A} 11=\left(1 / \mathrm{E} 1 \_ \text {avg*de du_w1o1 }\right)^{\wedge} 2 ; \mathrm{A} 12=\left(1 / \mathrm{E} 2 \_ \text {avg*de du_w2o1 }\right)^{\wedge} 2 ; \mathrm{A} 13=\left(1 / \mathrm{E} 3 \_ \text {avg*de du } \_ \text {w } 301\right)^{\wedge} 2$;

$\mathrm{A} 14=\left(1 / \mathrm{E} 4 \_ \text {avg*de_du_w2o2 }\right)^{\wedge} 2 ; \mathrm{A} 15=\left(1 / \mathrm{E} 5 \_ \text {avg*de_du_w2o2 }\right)^{\wedge} 2 ; \mathrm{A} 16=\left(1 / \mathrm{E} 6 \_ \text {avg*de_du_w3o2 }\right)^{\wedge} 2$;

$\mathrm{A} 21=\left(1 /\left(\mathrm{E} 1 \_ \text {avg*U}\right)^{*} \text { de_dt_w1o1 }\right)^{\wedge} 2 ; \mathrm{A} 22=\left(1 /\left(\mathrm{E} 2 \_a v g * \mathrm{U}\right) * \mathrm{de} \_\mathrm{dt} \_\mathrm{w} 2 \mathrm{o} 1\right)^{\wedge} 2 ; \mathrm{A} 23=\left(1 /\left(\mathrm{E} 1 \_a v g * \mathrm{U}\right) * \mathrm{de}\right.$ dt $\mathrm{w} 3 \mathrm{o} 1)^{\wedge} 2$;

A24=(1/(E4_avg*U)*de_dt_w1o2)^2;A25=(1/(E5_avg*U)*de_dt_w2o2)^2;A26=(1/(E6_avg*U)*de_dt_ $\mathrm{w} 3 \mathrm{o} 2)^{\wedge} 2$;

$\mathrm{A} 31=\left(1 / \mathrm{E} 1 \_\mathrm{avg} * \mathrm{de} \_\mathrm{dr} \_\mathrm{w} 1 \mathrm{o} 1\right)^{\wedge} 2 ; \mathrm{A} 32=\left(1 / \mathrm{E} 2 \mathrm{avg} * \mathrm{de} \_\mathrm{dr} \_\mathrm{w} 2 \mathrm{o} 1\right)^{\wedge} 2 ; \mathrm{A} 33=\left(1 / \mathrm{E} 3 \_\mathrm{avg} * \mathrm{de} \_\mathrm{dr} \_\mathrm{w} 3 \mathrm{o} 1\right)^{\wedge} 2 ;$

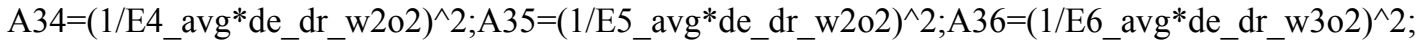

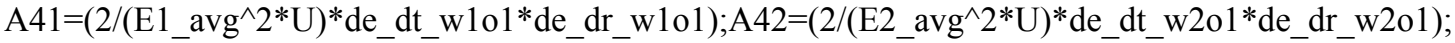
$\mathrm{A} 43=\left(2 /\left(\mathrm{E} 3 \_\mathrm{avg}{ }^{\wedge} 2 * \mathrm{U}\right) * \mathrm{de} \_\mathrm{dt} \_\mathrm{w} 3 \mathrm{o} 1 * \mathrm{de} \_\mathrm{dr} \_\mathrm{w} 3 \mathrm{o} 1\right) ; \mathrm{A} 44=\left(2 /\left(\mathrm{E} 4{ }_{-}\right.\right.$avg $\left.\left.{ }^{\wedge} 2 * \mathrm{U}\right) * \mathrm{de} \_\mathrm{dt} \_\mathrm{w} 1 \mathrm{o} 2 * \mathrm{de} \_\mathrm{dr} \_\mathrm{w} 1 \mathrm{o} 2\right)$; $\mathrm{A} 45=\left(2 /\left(\mathrm{E} 5 \_\mathrm{avg}{ }^{\wedge} 2 * \mathrm{U}\right) * \mathrm{de}_{-} \mathrm{dt} \_\mathrm{w} 2 \mathrm{o} 2 * \mathrm{de}_{-} \mathrm{dr}_{-} \mathrm{w} 2 \mathrm{o} 2\right) ; \mathrm{A} 46=\left(2 /\left(\mathrm{E} 6 \_\mathrm{avg}{ }^{\wedge} 2 * \mathrm{U}\right) * \mathrm{de}_{-} \mathrm{dt} \_\mathrm{w} 3 \mathrm{o} 2 * \mathrm{de}_{-} \mathrm{dr}_{-}{ }^{-} \mathrm{w} 3 \mathrm{o} 1\right) ;$

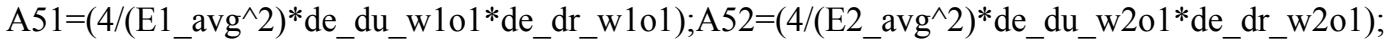

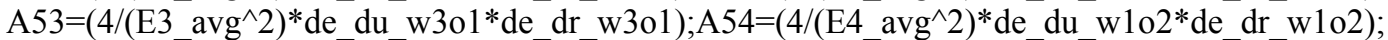
$\mathrm{A} 55=\left(4 /\left(E 5 \_a v g \wedge 2\right) * d e \_d u \_w 202 * d e \_d r \_w 2 o 2\right) ; A 56=\left(4 /\left(E 6 \_a v g \wedge 2\right) * d e \_d u \_w 3 o 2 * d e \_d r \_w 3 o 1\right)$;

A61 $=\left(4 /\left(E 1 \_a v g \wedge 2 * U\right) * d e \_d u \_w 101 * d e\right.$ dt_w1o1);A62 $=\left(4 /\left(E 2 \_a v g \wedge 2 * U\right) * d e \_d u \_w 2 o 1 * d e\right.$ dt_w2o1);

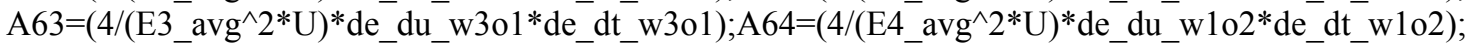
$\mathrm{A} 65=\left(4 /\left(\mathrm{E} 5 \_\right.\right.$avg $\left.\left.2 * \mathrm{U}\right) * \mathrm{de}_{-} \mathrm{du} \_\mathrm{w} 2 \mathrm{o} 2 * \mathrm{de}_{-} \mathrm{dt} \_\mathrm{w} 2 \mathrm{o} 2\right) ; \mathrm{A} 66=\left(4 /\left(\mathrm{E} 6 \_\mathrm{avg}{ }^{\wedge} 2 * \mathrm{U}\right) * \mathrm{de}_{-} \mathrm{du}_{-} \mathrm{w} 3 \mathrm{o} 2 * \mathrm{de}_{-} \mathrm{dt} \_\mathrm{w} 3 \mathrm{o} 2\right) ;$

$\mathrm{A}=[\mathrm{A} 11$ A12 A13 A14 A15 A16; A21 A22 A23 A24 A25 A26; A31 A32 A33 A34 A35 A36; A41 A42 A43 A44 A45 A46; A51 A52 A53 A54 A55 A56; A61 A62 A63 A64 A65 A66];

\% Evaluate coefficients of B matrix (Refer to Equation 4.24)

$\mathrm{B} 1=\left(\mathrm{E} 1 \_\mathrm{rms} / \mathrm{E} 1 \_\mathrm{avg}\right)^{\wedge} 2 ; \mathrm{B} 2=\left(\mathrm{E} 2 \_\mathrm{rms} / \mathrm{E} 2 \_\mathrm{avg}\right)^{\wedge} 2 ; \mathrm{B} 3=\left(\mathrm{E} 3 \_\mathrm{rms} / \mathrm{E} 3 \_\mathrm{avg}\right)^{\wedge} 2 ;$

$\mathrm{B} 4=\left(\mathrm{E} 4 \_\mathrm{rms} / \mathrm{E} 4 \_\mathrm{avg}\right)^{\wedge} 2 ; \mathrm{B} 5=\left(\mathrm{E} 5 \_ \text {rms/E5_avg }\right)^{\wedge} 2 ; \mathrm{B} 6=\left(\mathrm{E} 6 \_\mathrm{rms} / \mathrm{E} 6 \_ \text {avg }\right)^{\wedge} 2 ;$

$\mathrm{B}=[\mathrm{B} 1 ; \mathrm{B} 2 ; \mathrm{B} 3 ; \mathrm{B} 4 ; \mathrm{B} 5 ; \mathrm{B} 6] ;$

$\%$ Solve for parameters (invert matrix : Equation 4.24)

$\mathrm{c}=\operatorname{inv}(\mathrm{A}) * \mathrm{~B}$

u_sq $=\mathrm{c}(1)$

$\mathrm{v \_ sq}=\mathrm{c}(2)$

rho_sq $=\mathrm{c}(3)$

rho_v $=\mathrm{c}(4)$

rho $\mathrm{u}=\mathrm{c}(5)$

$\mathrm{u}_{-} \mathrm{v}=\mathrm{c}(6)$ 


\section{APPENDIX E}

\section{TWO FLUID INTERFACE IN ABSENCE OF SHEAR \& BUOYANCY}

Figure E.1 shows a photograph of the interface between the two air streams flowing at a velocity of $0.6 \mathrm{~m} / \mathrm{s}$. It is seen that the interface is reasonably flat. It is observed that the thickness of the mixing layer formed by the wake is $\sim 8 \mathrm{~cm}$ at a location $1.75 \mathrm{~m}$ downstream from the splitter plate. In case of the low Atwood number R-T run $\left(A_{\mathrm{t}} \#\right.$ $0.035, U_{\mathrm{m}}=0.6 \mathrm{~m} / \mathrm{s}$ ), the thickness of the mixing layer at the same location is $\sim 64 \mathrm{~cm}$. Figures E.2, E.3 and E.4 are images of the interface at velocities $U_{\mathrm{m}}=0.84 \mathrm{~m} / \mathrm{s}, 1.2 \mathrm{~m} / \mathrm{s}$ and $1.65 \mathrm{~m} / \mathrm{s}$ respectively; which corresponds to the mean velocities for Atwood number $0.097,0.259$ and 0.47 experimental runs. It is observed that the thickness of the mixing layer formed by the wake at a location $1.75 \mathrm{~m}$ from the splitter plate is between $7 \mathrm{~cm}$ to $9 \mathrm{~cm}$ for all the cases. Thus the mixing layer formed by the wake is much smaller compared with that formed by the buoyancy driven Rayleigh-Taylor mixing. Since addition of smoke to the air stream causes a small temperature/density difference, smoke is added to the top air stream so that a stable buoyancy configuration is maintained. The fuzziness observed in the interface is because the image is a line-of-sight average of the three-dimensional structures present at the interface along the depth of the channel. All images are taken with a Nikon D70s camera which is manually set at F/6.3, shutter speed of $1 / 3200 \mathrm{~s}$, and focal length of $70 \mathrm{~mm}$. 


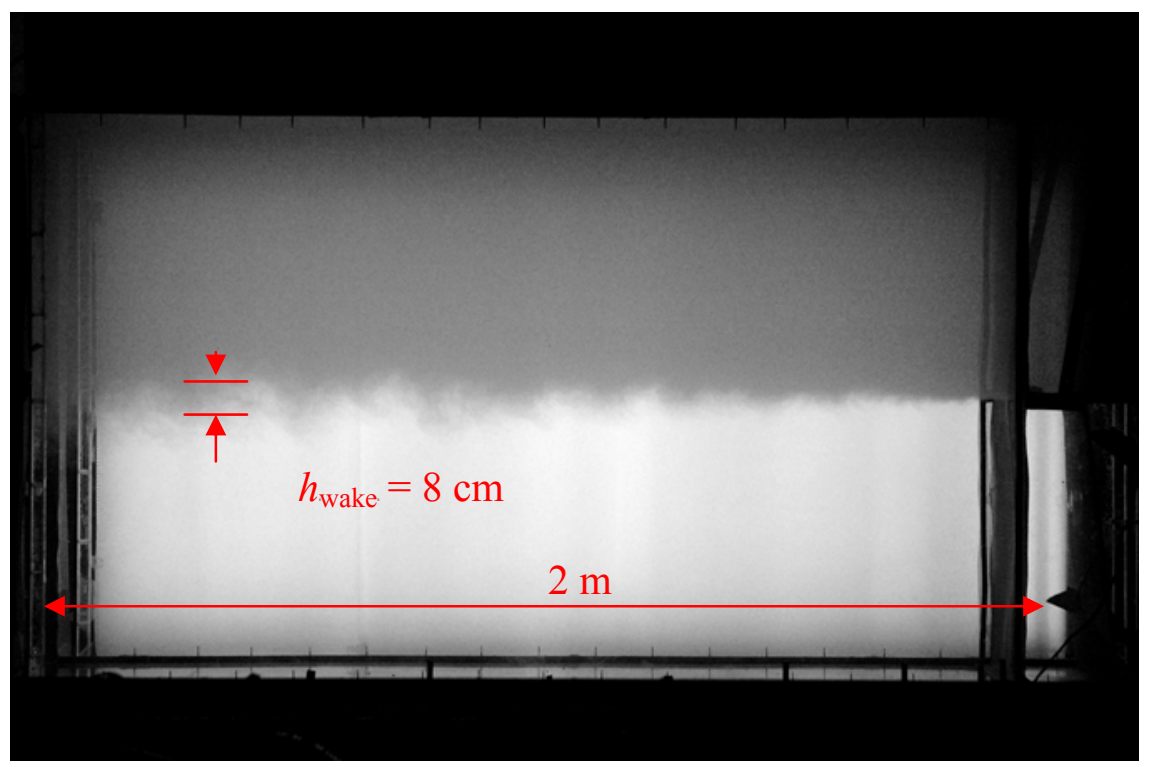

Figure E.1 Two fluid interface in absence of shear and density gradient $\left(U_{\mathrm{m}}=0.6 \mathrm{~m} / \mathrm{s}\right)$. Picture of the whole channel showing that the mix layer formed by the wake.

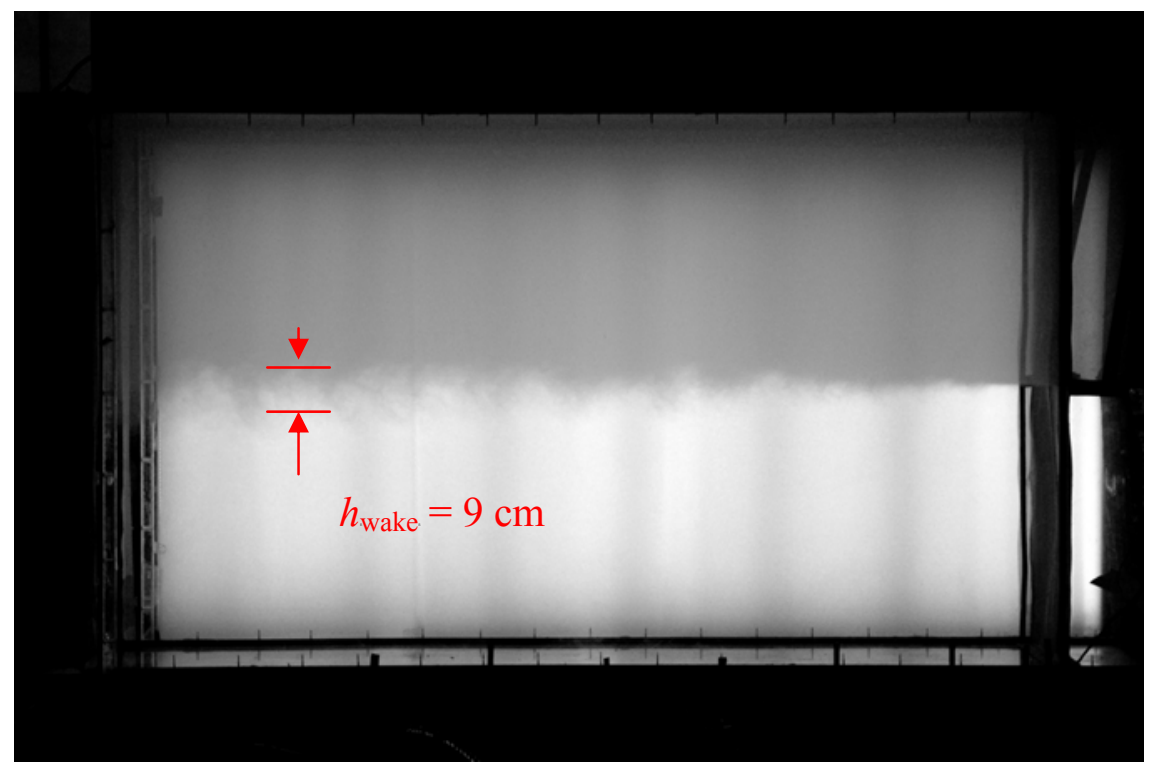

Figure E.2 Two fluid interface in absence of shear and density gradient $\left(U_{\mathrm{m}}=0.84 \mathrm{~m} / \mathrm{s}\right)$. The mean velocity corresponds to an $A_{\mathrm{t}} \# 0.097$ experimental run. 


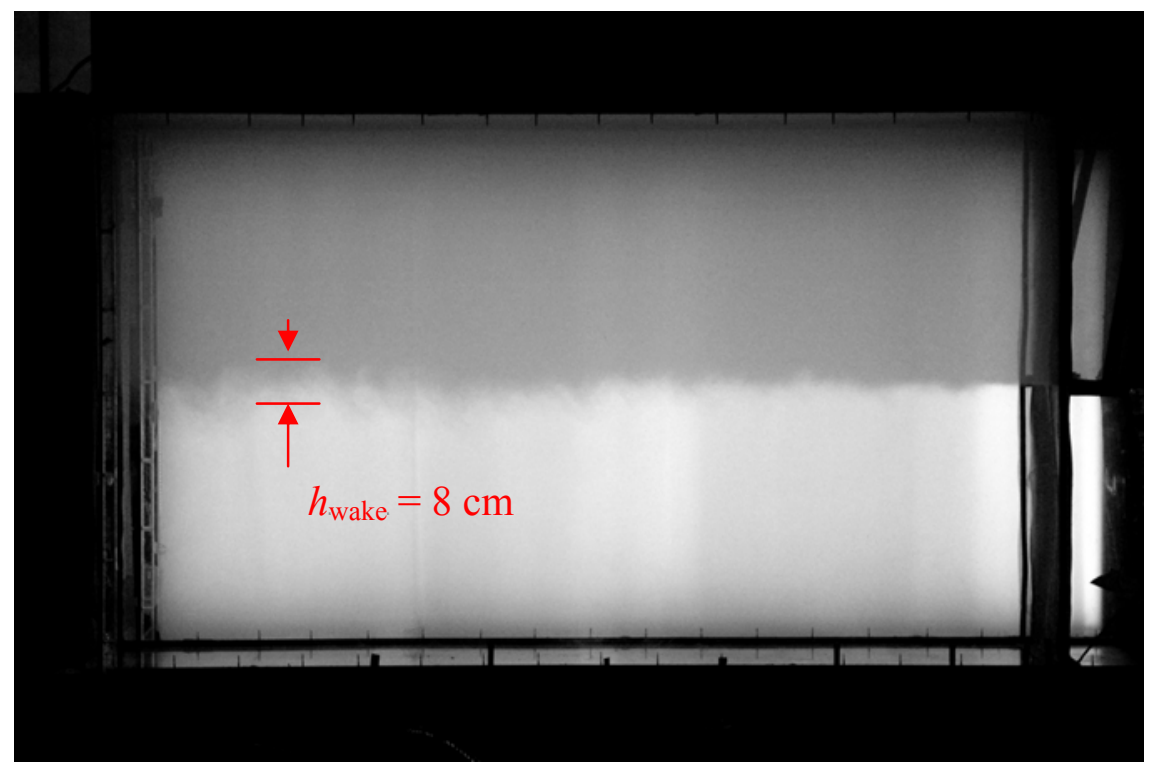

Figure E.3 Two fluid interface in absence of shear and density gradient $\left(U_{\mathrm{m}}=1.2 \mathrm{~m} / \mathrm{s}\right)$. The mean velocity corresponds to an $A_{\mathrm{t}} \# 0.259$ experimental run.

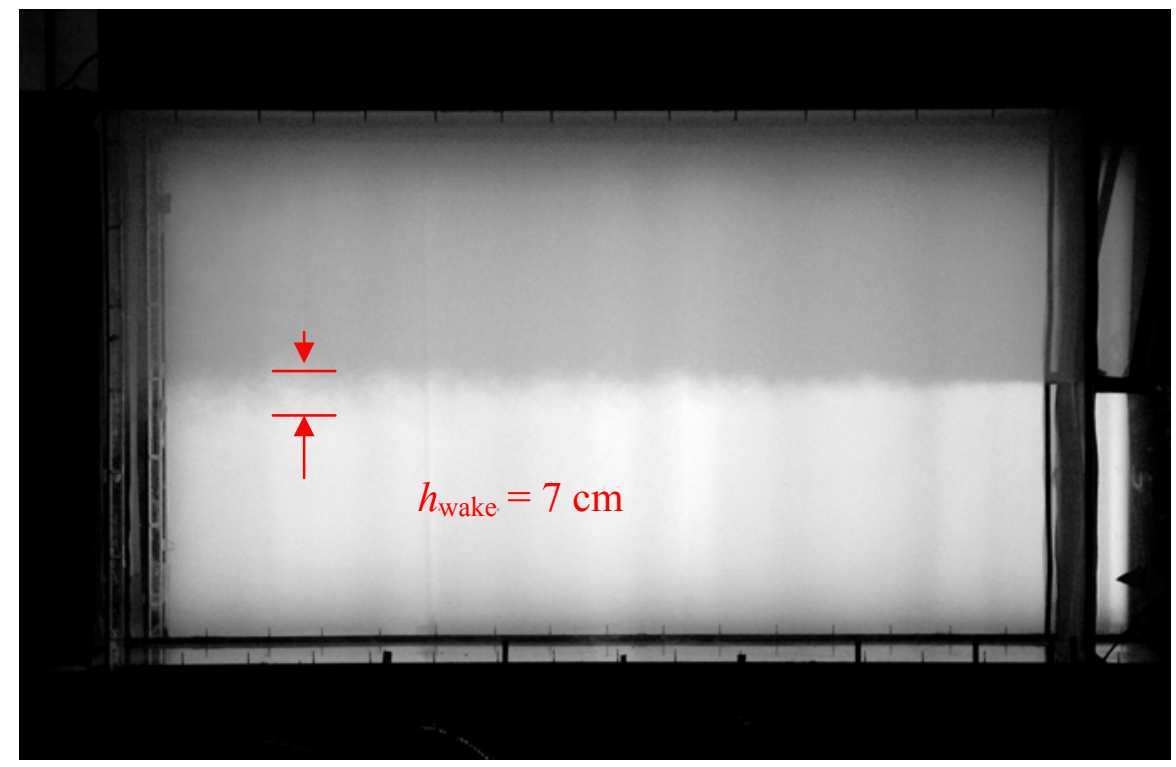

Figure E.4 Two fluid interface in absence of shear and density gradient $\left(U_{\mathrm{m}}=1.65 \mathrm{~m} / \mathrm{s}\right)$. The mean velocity corresponds to an $A_{\mathrm{t}} \# 0.47$ experimental run. 


\section{VITA}

Name:

Place of birth:

Permanent Address:

Education:
Arindam Banerjee

Calcutta (Kolkata), West Bengal

India

164/78 Lake Gardens, Flat B-10

Calcutta 700045. India

B.E. (Mechanical Engineering), June 1999

Jadavpur University, Calcutta, India

M.S. (Mechanical Engineering), August 2002

Florida Institute of Technology, Melbourne, Florida

Ph.D. (Mechanical Engineering), August 2006

Texas A\&M University, College Station, Texas 